Universidade de Brasília

Instituto de Ciências Exatas

Departamento de Matemática

Efeitos combinados de não-linearidades côncavas e convexas em alguns problemas elípticos.

\author{
por \\ Bertha Katherine Rodríguez Chávez
}

Brasília 


\section{Efeitos combinados de não-linearidades côncavas e convexas em alguns problemas elípticos.}

por

Bertha Katherine Rodríguez Chávez *

Dissertação apresentada ao Departamento de Matemática da Universidade de Brasília, como parte dos requisitos para obtenção do grau de

MESTRE EM MATEMÁTICA

Brasília, 04 de Março de 2015

Comissão Examinadora:

Dr. Ricardo Ruviaro - UnB - Orientador

Dr. Jiazheng Zhou - UnB - Examinador

Dr. Reinaldo de Marchi - UFMT- Examinador

*O autor foi bolsista do CNPq durante a elaboração deste trabalho. 
Aos meus pais, Noel e Flor. 


\section{Agradecimentos}

Primeiramente a Deus por ter me concedido saúde e capacidade sobre todas as coisas nos momentos mais importantes dessa etapa de minha vida para conquistar meu sonho.

À minha família. Ao meu pai Noel, por todo o apoio, carinho e pelas palavras de força. À minha mãe Flor, fonte inesgotável de amor, por ter compreendido a distância. Os meus irmãos queridos, Diego e Isabel, por terem me dado muito carinho e afeto nos momentos que estivemos juntos. E por serem minha inspiração para continuar.

À meu namorado Ricardo pelo carinho e amor compartilhado durante quatro anos, caminhando juntos na consecução dos nossos objetivos. Sendo o meu apoio a cada passo que eu tenho tido desde o início de esta longa jornada.

Ao meu orientador Ricardo Ruviaro pela dedicação, paciência, atencão, comprensão e pelo exemplo de profissional que se tornou para mim. É mais que um professor, é um amigo.

Aos professores da banca Jiazheng Zhou e Reinaldo de Marchi pelas valiosas contribuições, enriquecendo ainda mais essa dissertação.

Aos professores da Pós-Graduação que me ajudaram a percorrer essa difícil trajetória em busca de conhecimento.

A todos os que fazem parte do Departamento de Matemática da UnB.

A todos meus amigos da UnB pelo companheirismo, motivação e pelos momentos compartilhados.

Agradeço ao $\mathrm{CNPq}$ pelo apoio financeiro à este trabalho. 


\section{Resumo}

Neste trabalho estudaremos a existência, não existência e multiplicidade de soluções positivas para a família de problemas

$$
\left\{\begin{aligned}
-\Delta u & =f_{\lambda}(x, u), & & x \in \Omega, \\
u & >0, & & x \in \Omega, \\
u & =0, & & x \in \partial \Omega,
\end{aligned}\right.
$$

onde $f_{\lambda}: \Omega \times \mathbb{R} \rightarrow \mathbb{R}, \lambda>0$ é um parâmetro, $\Omega \subset \mathbb{R}^{N}$ um domínio limitado com $N \geq 3$.

Os principais resultados utilizados são o Teorema do Passo da Montanha e o método de sub e supersolução.

Palavras-Chaves: Equações semilineares; não linearidades do tipo côncavo-convexas, Teorema do Passo da Montanha; sub e supersolução. 


\section{Abstract}

In this work we study the existence, non-existence and multiplicity of positive solutions for the family of elliptic problem

$$
\left\{\begin{aligned}
-\Delta u & =f_{\lambda}(x, u), & & x \in \Omega, \\
u & >0, & & x \in \Omega, \\
u & =0, & & x \in \partial \Omega,
\end{aligned}\right.
$$

where $f_{\lambda}: \Omega \times \mathbb{R} \rightarrow \mathbb{R}, \lambda>0$ is a real parameter, $\Omega \subset \mathbb{R}^{N}$ is a bounded domain with $N \geq 3$.

To show the main results we used The Mountain Pass Theorem and The Sub and Supersolution.

Keywords: Semilinear equations, non-linearities of the concave-convex type, The Mountain Pass Theorem, Sub and Supersolution. 


\section{Sumário}

1 Existência de Soluções Positivas 5

1.1 Existência de uma solução pelo método de sub e supersolução . . . . . . . . . . . . . 5

1.2 Existência de uma segunda solucão por argumentos variacionais . . . . . . . . . . . . . . 21

1.3 Existência de infinitas soluções . . . . . . . . . . . . . . . . . . . . . . . . 32

2 Multiplicidade de Soluções $\quad 36$

2.1 Existência de uma solução sem condição de crescimento . . . . . . . . . . . . . . . 40

2.2 Não existência de soluções para $\lambda$ grande . . . . . . . . . . . . . . . . . . . . . . 43

2.3 Existência de uma solução para $\lambda=\Lambda \ldots \ldots \ldots \ldots \ldots$. . . . . . . . . . . 44

2.4 Existência de uma segunda solução no caso subcrítico . . . . . . . . . . . . . . 45

2.5 Existência de uma segunda solução no caso crítico com $\sigma<1 \ldots \ldots \ldots$

2.6 Existência de uma segunda solução para o caso crítico com $\sigma<2^{*}-1 \ldots \ldots$. . . . . 55

2.7 Aplicações . . . . . . . . . . . . . . . . . . . . . . . 5 57

$\begin{array}{lr}\text { A Resultados Importantes } & 64\end{array}$

A.1 Regularidade . . . . . . . . . . . . . . . . . . . . . 68

A.2 $I_{\lambda}$ satisfaz a condição $(P S) \ldots \ldots \ldots \ldots \ldots \ldots \ldots$

A.3 A constante de Sobolev . . . . . . . . . . . . . . . . . . 73

A.4 Funcionais com simetria e teoria de índice . . . . . . . . . . . . . . . . . . . 85

B Teorema do Passo da Montanha $\quad 87$

B.1 Lema de Deformação Quantitativo . . . . . . . . . . . . . . . . . . . 87

B.1.1 Campo Pseudo-Gradiente . . . . . . . . . . . . . . 87

B.2 O Teorema do Passo da Montanha . . . . . . . . . . . . . . . . . . 94 
C Sub e supersolução

C.1 Sub e supersolucão fraca . . . . . . . . . . . . . . . . . . . . . . . . . . . . . . . . . 102

Bibliografia 


\section{Notações}

Neste trabalho, fazemos uso das seguintes notações:

- $M, C, C_{0}, C_{1}, \ldots$ denotam constantes positivas (possivelmente diferentes).

- $\int_{\mathbb{R}^{N}} f(x) d x$ representada por $\int_{\mathbb{R}^{N}} f$.

- $B_{R}(p)$ denota a bola aberta de raio $R$ com centro no ponto $p \in \mathbb{R}^{N}$; e $\partial B_{R}(p)$ denota a fronteira desta bola.

- $\mathbb{R}^{+}=[0,+\infty)$.

- Representaremos por $\langle\cdot, \cdot\rangle$ o par dualidade entre os espaçõs $E$ e seu dual $E^{\prime}$.

- Representaremos a convergência fraca em $E$ por " $\rightarrow$ " e a convergência forte por " $\rightarrow$."

- Representaremos por $u_{n} \uparrow u$, como $u_{n}$ sendo uma sequência crescente que converge para $u$.

- Representaremos por $u_{n} \downarrow u$, como $u_{n}$ sendo uma sequência decrescente que converge para $u$.

- $\operatorname{supp} \varphi$ denota o suporte da função $\varphi$.

- $|\Omega|$ ou meas $(\Omega)$ denota a medida de Lebesgue de um conjunto mensurável $\Omega \subset \mathbb{R}^{N}$.

- $\chi_{\Omega}$ denota a função característica do conjunto $\Omega$.

- $H$ denota $H_{0}^{1,2}(\Omega)$, dotado com a norma $\|u\|^{2}=\int_{\Omega}|\nabla u|^{2} d x$.

- $\|\cdot\|$ denota a norma do espaço $H$.

- Para $1 \leq p<N, p^{*}=\frac{N p}{N-p}$ é o expoente crítico de Sobolev.

- $A=O(x)$ quando $\frac{A}{x} \leq M$, para alguma constante $M>0$.

- $A_{n}=o_{n}(x)$ se $\frac{A_{n}}{x} \rightarrow 0$ quando $n \rightarrow \infty$.

- $u^{+}(x)=\max \{u(x), 0\}$.

- $u^{-}(x)=\min \{u(x), 0\}$. 
- $\nabla u=\left(\frac{\partial u}{\partial x_{1}}, \frac{\partial u}{\partial x_{2}}, \cdots, \frac{\partial u}{\partial x_{N}}\right)$ é o gradiente da função $u$.

- $\Delta u=\sum_{i=1}^{N} \frac{\partial^{2} u}{\partial x_{i}^{2}}$ é o Laplaciano da função $u$.

- $C(\Omega)=C(\Omega, \mathbb{R})$ denota o espaço das funções contínuas no $\Omega$ e $C_{0}(\Omega)$ são funções contínuas de suporte compacto em $\Omega$.

- $C^{k}(\Omega), k \geq 1$ inteiro, denota o espaço das funções $k$ vezes continuamente diferenciáveis sobre $\Omega \mathrm{e}$ $C^{\infty}(\Omega)=\bigcap_{k \geq 1} C^{k}(\Omega)$.

- $C_{0}^{k}(\Omega)=C^{k}(\Omega) \cap C_{0}(\Omega)$ e $C_{0}^{\infty}(\Omega)=C^{\infty} \cap C_{0}(\Omega)$.

- $C^{0, \beta}(\bar{\Omega})=\left\{u \in C(\Omega): \sup _{x, y \in \Omega} \frac{|u(x)-u(y)|}{|x-y|^{\beta}}<\infty\right\} \operatorname{com} 0<\beta<1$, e $C^{k, \beta}(\bar{\Omega})$ são as funções em $C^{k}(\Omega)$ tais que todas as derivadas parciais até a ordem $k$ estão em $C^{0, \beta}$.

- $L^{p}(\Omega)=\left\{u: \Omega \rightarrow \mathbb{R}\right.$ mensurável $\left.: \int_{\Omega}|u|^{p} d x<\infty\right\}$ em que $1 \leq p<\infty$ e $\Omega \in \mathbb{R}^{N}$ é um aberto conexo, com norma dada por

$$
\|u\|_{p}:=\left(\int_{\Omega}|u|^{p} d x\right)^{\frac{1}{p}}
$$

- $L^{\infty}(\Omega)$ denota o espaço de funções mensuráveis que são limitadas quase sempre em $\Omega$ com norma dada por

$$
\|u\|_{\infty}:=\inf \{C>0:|u(x)| \leq C \text { quase sempre em } \Omega\}
$$

- $D^{1,2}\left(\mathbb{R}^{N}\right):=\left\{u \in L^{2^{*}}\left(\mathbb{R}^{N}\right):|\nabla u| \in L^{2}\left(\mathbb{R}^{N}\right)\right\}$ munido da norma $\|\nabla u\|_{2}$.

- Para $1 \leq p<\infty$,

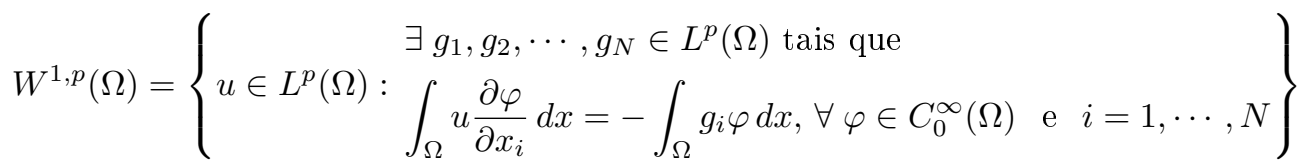

com norma dada por

$$
\|u\|_{1, p}=\left[\int_{\Omega}\left(|\nabla u|^{p}+|u|^{p}\right) d x\right]^{\frac{1}{p}}
$$

e

$$
W^{2, p}(\Omega)=\left\{u \in W^{1, p}(\Omega): \frac{\partial u}{\partial x_{i}} \in W^{1, p}(\Omega), \text { para todo } i=1, \cdots, N\right\} \text {. }
$$

- O primeiro autovalor de

$$
\left\{\begin{aligned}
-\Delta \varphi & =\lambda \varphi, & & x \in \Omega, \\
\varphi & =0, & & x \in \partial \Omega
\end{aligned}\right.
$$

é denotado por $\lambda_{1}, \varphi_{1}$ denota a correspondente autofunção satisfazendo $\varphi_{1}>0$ em $\Omega$ e tal que $\left\|\varphi_{1}\right\|_{2}=1$. 


\section{Introdução}

Nessa dissertação faremos um estudo baseados em dois artigos, primeiramente estudaremos o artigo de Ambrosetti, Brezis e Cerami [1] e na sequência o artigo apresentado por de Figueiredo, Gossez e Ubilla [12], sobre a existência, não existência e multiplicidade de soluções positivas para a família de problemas

$$
\left\{\begin{aligned}
-\Delta u & =f_{\lambda}(x, u), & & x \in \Omega, \\
u & >0, & & x \in \Omega, \\
u & =0, & & x \in \partial \Omega,
\end{aligned}\right.
$$

onde $\Omega \subset \mathbb{R}^{N}$ é um domínio suave e limitado, $N \geq 3$ e $f_{\lambda}: \Omega \times \mathbb{R} \rightarrow \mathbb{R}$ é uma função de Carathéodory, ou seja,

(i) $s \mapsto f(x, s)$ é contínua para quase todo $x \in \Omega$;

(ii) $x \mapsto f(x, s)$ é mensurável para todo $s \in \mathbb{R}$.

Esta dissertação está dividida em dois capítulos e três apêndices organizados da seguinte forma.

No Capítulo 1, estudaremos o trabalho de Ambrosetti, Brezis e Cerami [1]. Para isso consideramos o problema

$$
\left(P_{0}\right) \quad\left\{\begin{aligned}
-\Delta u & =\lambda u^{q}+u^{p}, & & x \in \Omega, \\
u & >0, & & x \in \Omega, \\
u & =0, & & x \in \partial \Omega,
\end{aligned}\right.
$$

onde $0<q<1<p$. Este capítulo, esta dividido em três seções. Na primeira seção, estudaremos o Teorema 1.1 que trata da existência de uma solução positiva via sub e supersolução para o problema $\left(P_{0}\right)$ para todo $\lambda \in(0, \Lambda)$, garantimos ainda nesse teorema que o problema $\left(P_{0}\right)$ têm ao menos uma solução quando $\lambda=\Lambda$ e fechamos a sua prova, mostrando que o problema $\left(P_{0}\right)$ não possui solução quando $\lambda>\Lambda$.

Na sequência demonstraremos o Teorema 1.2 que garante a existência de um $A>0$, verificando que o problema $\left(P_{0}\right)$ tem no máximo uma solução quando $\lambda \in(0, \Lambda)$.

Dando continuidade ao estudo, nesse capítulo na seção 2 estudaremos o Teorema 1.3 que trata da existência de uma segunda solução por argumentos variacionais para o problema $\left(P_{0}\right)$ quando $\lambda \in(0, \Lambda)$.

Por fim, na seção 3 deste capítulo apresentamos o Teorema 1.4 que garante a existência de infinitas soluções para o problema $\left(P_{0}\right)$ para todo $\lambda$ pequeno, isto é, $\lambda \in\left(0, \lambda^{*}\right)$.

No Capítulo 2, estudamos o trabalho de Figueiredo, Gossez e Ubilla [12], onde trataremos a existência, 
não-existência e multiplicidade de soluções para a família de problemas

$$
(P) \quad\left\{\begin{aligned}
-\Delta u & =f_{\lambda}(x, u), & & x \in \Omega, \\
u & >0, & & x \in \Omega, \\
u & =0, & & x \in \partial \Omega,
\end{aligned}\right.
$$

com $\Omega$ um domínio limitado em $\mathbb{R}^{N}, N \geq 3$ e $\lambda>0$ um parâmetro.

Este capítulo esta dividido em sete seções. Na primeira seção, estudaremos o Teorema 2.1 que sobre certas hipóteses, como por exemplo, sem a condição de crescimento, teremos que o problema $(P)$ tem ao menos uma solução para $0<\lambda<\Lambda$ e nenhuma solução para $\lambda>\Lambda$.

Na sequência, para a segunda seção apresentaremos a prova do Teorema 2.2 onde mostraremos a não existência de solução para o problema $(P)$, quando $\lambda$ grande, além de verificarmos que $\Lambda<\infty$.

Na terceira seção, apresentaremos a prova do Teorema 2.3, onde sob certas hipóteses garantimos a existência de uma solução para o problema $(P)$ quando $\lambda=\Lambda$.

Para a quarta seção, daremos a prova do Teorema 2.4, onde mostraremos sob certas hipóteses a existência de uma segunda solução para o problema $(P)$ no caso subcrítico. Mostrando assim que o problema $(P)$ tem ao menos duas soluções $u, v$ para $0<\lambda<\Lambda$, com $u<v$ em $\Omega$.

Para a próxima seção, faremos a prova do Teorema 2.5 mostrando a existência de uma segunda solução no caso crítico, onde alguns termos poderão mudar de sinal, sobre essas condições e algumas hipóteses, mostraremos que o problema $(P)$ tem ao menos duas soluções $u, v$ para $0<\lambda<\Lambda$, com $u<v$ em $\Omega$.

Para a sexta e penúltima seção desse capítulo, apresentaremos a prova do Teorema 2.6, mostrando a existência de uma segunda solução no caso crítico, com algumas restrições no sinal de alguns termos e hipóteses um pouco distintas do teorema anterior, garantimos também que o problema $(P)$ tem ao menos duas soluções $u, v$ para $0<\lambda<\Lambda$, com $u<v$ em $\Omega$.

Na última seção, seção 2.7 apresentaremos algumas aplicações dos resultados apresentados anteriormente.

No Apêndice $A$ apresentaremos resultados úteis para o bom entendimento da dissertação, destacamos como por exemplo: regularidade, princípio do máximo, desigualdades técnicas.

Para o Apêndice $B$, apresentamos com todos os detalhes a prova do Teorema do Passo da Montanha, utilizando o Lema de Deformação, Campo Pseudo-Gradiente, dentre outros fatos importantes para a prova do Teorema.

Por fim no Apêndice $C$, faremos um estudo sobre a definição e resultados de sub e supersolução. 


\section{Capítulo}

\section{Existência de Soluções Positivas}

Neste capítulo consideramos o seguinte problema

$$
\left(P_{\lambda}\right) \quad\left\{\begin{aligned}
-\Delta u & =\lambda u^{q}+u^{p}, & & x \in \Omega, \\
u & >0, & & x \in \Omega, \\
u & =0, & & x \in \partial \Omega,
\end{aligned}\right.
$$

onde $\Omega$ é um domínio limitado em $\mathbb{R}^{N}, 0<q<1<p \leq(N+2) /(N-2):=2^{*}-1$, com fronteira suave $\partial \Omega, N \geq 3, \Delta$ é o operador Laplaciano e $\lambda$ é um parâmetro real.

Dizemos que $u \in H, u \neq 0$ em $\Omega$ é uma solução fraca para o problema $\left(P_{\lambda}\right)$, se

$$
\int_{\Omega} \nabla u \nabla v d x=\lambda \int_{\Omega}|u|^{q-1} u v d x+\int_{\Omega}|u|^{p-1} u v d x
$$

O funcional associado ao problema $\left(P_{\lambda}\right)$ é $I_{\lambda}: H \rightarrow \mathbb{R}$ dado por

$$
I_{\lambda}(u)=\frac{1}{2}\|u\|^{2}-\frac{\lambda}{q+1} \int_{\Omega}|u|^{q+1} d x-\frac{1}{p+1} \int_{\Omega}|u|^{p+1} d x,
$$

onde $I_{\lambda}$ denota a energia de $u$ e $I_{\lambda} \in C^{1}(H, \mathbb{R})$, assim

$$
I_{\lambda}^{\prime}(u) v=\int_{\Omega} \nabla u \nabla v d x-\lambda \int_{\Omega}|u|^{q-1} u v d x-\int_{\Omega}|u|^{p-1} u v d x, \quad \forall v \in H .
$$

Portanto, encontrar solução fraca para o problema $\left(P_{\lambda}\right)$ é equivalente a encontrar ponto crítico do funcional $I_{\lambda}$.

Agora, primeiramente mostraremos a existência de solução não trivial para o problema $\left(P_{\lambda}\right)$ mediante o método de sub e supersolução.

\subsection{Existência de uma solução pelo método de sub e supersolução}

Para um primeiro resultado temos a garantia da existência de uma constante positiva $\Lambda \in \mathbb{R}$ tal que uma solução de $\left(P_{\lambda}\right)$ existe se, e somente se, $0<\lambda<\Lambda$. Para encontrar tal solução usamos o método de 
sub e supersolução. O termo essencial aqui é $q$ e $p$ pode ser arbitrário.

Teorema 1.1. Para todo $0<q<1<p$ existe $\Lambda \in \mathbb{R}, \Lambda>0$, tal que:

1. Para todo $\lambda \in(0, \Lambda)$ o problema $\left(P_{\lambda}\right)$ tem uma solução mínima $u_{\lambda}$ tal que $I_{\lambda}\left(u_{\lambda}\right)<0$. Além disso, $u_{\lambda}$ é crescente com respeito a $\lambda$.

2. Para $\lambda=\Lambda$, o problema $\left(P_{\lambda}\right)$ têm ao menos uma solução fraca, $u \in H \cap L^{p+1}$.

3. Para todo $\lambda>\Lambda$, o problema $\left(P_{\lambda}\right)$ não têm solução.

Antes de demonstrarmos o Teorema 1.1, provaremos primeiramente alguns resultados adicionais que facilitaram a prova deste teorema. Inicialmente, considere o problema

$$
\left\{\begin{aligned}
-\Delta u & =f(u), & & x \in \Omega, \\
u & =0, & & x \in \partial \Omega,
\end{aligned}\right.
$$

onde $\Omega \subset \mathbb{R}^{N}, N \geq 1$, é um domínio regular e $f: \mathbb{R} \rightarrow \mathbb{R}$ uma função de classe $C^{\alpha}$. Dizemos que uma função $\underline{u} \in C^{2}(\Omega) \cap C(\bar{\Omega})$ é uma subsolução do problema (1.1) se

$$
\left\{\begin{aligned}
-\Delta \underline{u} \leq f(\underline{u}), & x \in \Omega, \\
\underline{u} \leq 0, & x \in \partial \Omega,
\end{aligned}\right.
$$

e dizemos que uma função $\bar{u} \in C^{2}(\Omega) \cap C(\bar{\Omega})$ é uma supersolução do problema (1.1) se

$$
\left\{\begin{aligned}
-\Delta \bar{u} & \geq f(\bar{u}), & & x \in \Omega, \\
\bar{u} & \geq 0, & & x \in \partial \Omega .
\end{aligned}\right.
$$

Agora definimos $\Lambda:=\sup \left\{\lambda>0:\left(P_{\lambda}\right)\right.$ tem uma solução $\}$.

Lema 1.1. $0<\Lambda<\infty$.

Demonstração. Seja e uma solução do problema

$$
\left\{\begin{aligned}
-\Delta e & =1, & & x \in \Omega, \\
e & =0, & & x \in \partial \Omega .
\end{aligned}\right.
$$

Uma vez que $0<q<1<p$, pode-se encontrar $\lambda_{0}>0$ tal que se $0<\lambda \leq \lambda_{0}$, então existe $M=M(\lambda)>0$ que satisfaz

$$
M \geq \lambda M^{q}\|e\|_{\infty}^{q}+M^{p}\|e\|_{\infty}^{p} .
$$

De fato, considere as funções

$$
\begin{aligned}
& f(x)=x \\
& g(x)=A x^{q}+B x^{p}, \quad \text { onde } \quad 0<q<1<p, \text { tal que } A=\lambda\|e\|_{\infty}^{q} \text { e } B=\|e\|_{\infty}^{p} .
\end{aligned}
$$

Temos,

$$
\begin{aligned}
g^{\prime}(x) & =q A x^{q-1}+p B x^{p-1}>0 \quad \text { para todo } \quad x>0 \\
g^{\prime \prime}(x) & =q(q-1) A x^{q-2}+p(p-1) B x^{p-2} \text { para todo } \quad x>0
\end{aligned}
$$


e $g(0)=0$. Assim, $g$ é côncava para cima em $[0,+\infty)$.

Logo, existe $c>0$ tal que $f(x) \geq g(x)$, para $0<x<c$. Portanto,

$$
f(x) \geq g(x) \Rightarrow x \geq A x^{q}+B x^{p}=\lambda\|e\|_{\infty}^{q} x^{q}+\|e\|_{\infty}^{p} x^{p},
$$

logo fazendo $x=M$ verificamos (1.4). A seguir é apresentado um esboço do comportamento das funções $f$ e $g$, para um melhor entendimento dessa desigualdade.

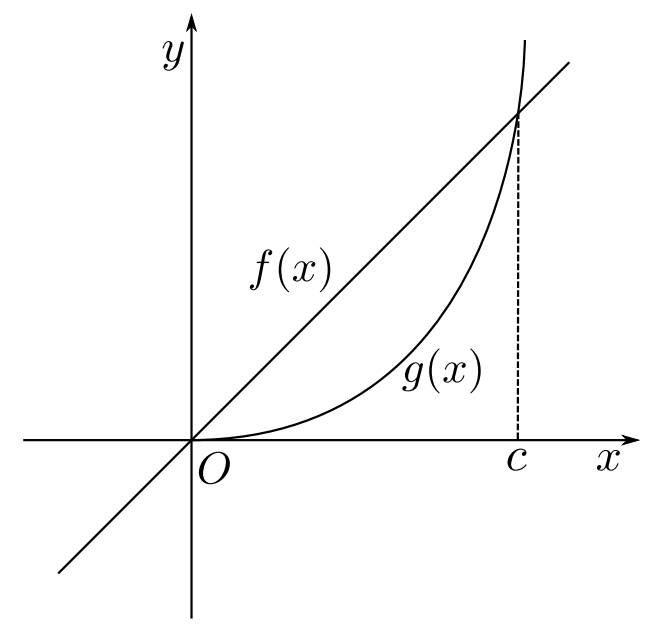

Como consequência temos que a função $M e$ verifica

$$
-\Delta(M e)=M(-\Delta e)=M \geq \lambda M^{q}\|e\|_{\infty}^{q}+M^{p}\|e\|_{\infty}^{p} \geq \lambda(M e)^{q}+(M e)^{p} .
$$

Assim, $M e$ é uma supersolução de $\left(P_{\lambda}\right)$. Mais ainda, para $\varepsilon$ suficientemente pequeno $\varepsilon \varphi_{1}$ é uma subsolução de $\left(P_{\lambda}\right)$, onde $\varphi_{1}$ é a autofunção do operador $-\Delta$ associada ao autovalor $\lambda_{1}$ em relação a $\Omega$.

De fato, temos que

$$
-\Delta\left(\varepsilon \varphi_{1}\right)=\varepsilon\left(\lambda_{1} \varphi_{1}\right)=\left(\frac{\varepsilon^{1-q} \lambda_{1}}{\lambda} \varphi_{1}^{1-q}\right) \lambda\left(\varepsilon \varphi_{1}\right)^{q} .
$$

Agora, basta determinarmos $\varepsilon>0$ tal que

$$
\left(\frac{\varepsilon^{1-q} \lambda_{1}}{\lambda} \varphi_{1}^{1-q}\right) \leq 1
$$

Como $\varphi_{1} \in L^{\infty}(\Omega)$, existe $C>0$ tal que

$$
\varphi_{1}^{1-q}(x) \leq C, \quad \forall x \in \bar{\Omega}
$$

Logo

$$
\left(\frac{\varepsilon^{1-q} \lambda_{1}}{\lambda} \varphi_{1}^{1-q}\right) \leq \varepsilon^{1-q}\left(\frac{\lambda_{1} C}{\lambda}\right) \leq 1 .
$$

Portanto, podemos considerar

$$
0<\varepsilon \leq\left(\frac{\lambda}{\lambda_{1} C}\right)^{\frac{1}{1-q}}
$$

o que implica que

$$
\varepsilon\left(\lambda_{1} \varphi_{1}\right)=-\Delta\left(\varepsilon \varphi_{1}\right) \leq \lambda\left(\varepsilon \varphi_{1}\right)^{q} \leq \lambda\left(\varepsilon \varphi_{1}\right)^{q}+\left(\varepsilon \varphi_{1}\right)^{p} .
$$


Assim, para $\varepsilon>0$ suficientemente pequeno e todo $\lambda>0$, temos que $\varepsilon \varphi_{1}$ é subsolução de $\left(P_{\lambda}\right)$.

Tomando $\varepsilon$ suficientemente pequeno, também temos que

$$
\varepsilon \varphi_{1}<M e .
$$

De fato, considere $\bar{u}$ uma supersolução positiva do problema $\left(P_{\lambda}\right)$. Seja $\varepsilon>0$ suficientemente pequeno e definamos o conjunto $\Omega_{1}:=\{x \in \Omega$, $\operatorname{dist}(x ; \partial \Omega) \leq \delta\}$. Como $\varphi_{1}, \bar{u}>0$ em $\Omega$ e pela compacidade de $\overline{\Omega \backslash \Omega_{1}}$, existe $C_{1}>0$ tal que

$$
\frac{\bar{u}(x)}{\varphi_{1}(x)} \geq C_{1}, \quad \forall x \in \overline{\Omega \backslash \Omega_{1}} .
$$

Por outro lado, pelo Lema de Hopf (ver Apêndice $A$, Lema $A .1$ ) temos que $\frac{\partial \bar{u}}{\partial \nu}<0$ em $\partial \Omega$, e como $\partial \Omega$ é compacto, temos que existe $C_{2}>0$ tal que

$$
\frac{\partial \bar{u}}{\partial \nu}(x)<C_{2}, \quad \forall x \in \bar{\Omega}_{1}
$$

Usando os mesmos argumentos, como $\varphi_{1} \in C_{0}^{1}(\bar{\Omega})$ temos que existe $C_{3}>0$ tal que

$$
\left|\frac{\partial \varphi_{1}}{\partial \nu}(x)\right| \leq C_{3}, \quad \forall x \in \bar{\Omega}_{1}
$$

Agora, considere a função $w: \bar{\Omega}_{1} \rightarrow \mathbb{R}$ definida por $w(x)=\gamma \varphi_{1}(x)-\bar{u}(x)$ com $\gamma>0$. Seja $C_{0}=$ $\inf _{\bar{\Omega}_{1}}\left(\partial \varphi_{1}\right) /(\partial \nu)$. Assim, se $\gamma>C_{1} / C_{0}$ temos

$$
\frac{\partial w}{\partial \nu}(x)=\gamma \frac{\partial \varphi_{1}}{\partial \nu}(x)-\frac{\partial \bar{u}}{\partial \nu}(x) \geq \gamma C_{0}-C_{2}>0, \quad \forall x \in \bar{\Omega}_{1}
$$

Fixando $x \in \bar{\Omega}_{1}$, definimos $g: \mathbb{R} \rightarrow \mathbb{R}$ por $g(t)=w(x+t \nu)$. Para cada $x \in \bar{\Omega}_{1}$, escolha um único $\tilde{x} \in \bar{\Omega}_{1}$ tal que a reta que passa por esses dois pontos coincida com a reta suporte do vetor normal exterior $\nu=\nu(\tilde{x})$. Assim, existe $\tilde{t}>0$ tal que $x+\tilde{t} \nu=\tilde{x} \in \partial \Omega$. Temos que $w(\partial \Omega) \equiv 0$, daí

$$
g(\tilde{t})=w(x+\tilde{t} \nu)=w(\tilde{x})=0 .
$$

Note que $g$ é a composta de duas funções de classe $C^{1}, \operatorname{logo} g:[0, \tilde{t}] \rightarrow \mathbb{R}$ é contínua em $[0, \tilde{t}]$ e derivável em $(0, \tilde{t})$, pelo Teorema do Valor Médio, existe $\xi \in(0, \tilde{t})$, tal que

$$
g(\tilde{t})-g(0)=g^{\prime}(\xi)(\tilde{t}-0) .
$$

Por outro lado, como

$$
\begin{aligned}
\frac{\partial w}{\partial \nu}(x+\xi \nu) & =\lim _{z \rightarrow 0} \frac{w(x+\xi \nu+z \nu)-w(x+\xi \nu)}{z} \\
& =\lim _{z \rightarrow 0} \frac{w(x+(\xi+z) \nu)-w(x+\xi \nu)}{z} \\
& =\lim _{z \rightarrow 0} \frac{g(\xi+z)-g(\xi)}{z} \\
& =g^{\prime}(\xi)
\end{aligned}
$$


Daí, por (1.7) temos

$$
\frac{\partial w}{\partial \nu}(x+\xi \nu) \tilde{t}=g^{\prime}(\xi) \tilde{t}=g(\tilde{t})-g(0)=-w(x) .
$$

Por (1.6) e sendo $\tilde{t}>0$ segue que

$$
-w(x)=\frac{\partial w}{\partial \nu}(x+\xi \nu) \tilde{t}>0, \quad \forall x \in \bar{\Omega}_{1} .
$$

Consequentemente, $w(x) \leq 0$ para todo $x \in \bar{\Omega}_{1}$ e assim $w(x)=\gamma \varphi_{1}(x)-\bar{u} \leq 0$. Segue então que

$$
\gamma \varphi_{1} \leq \bar{u}(x), \quad \forall x \in \bar{\Omega}_{1}
$$

o que implica que

$$
\frac{\bar{u}(x)}{\varphi_{1}(x)} \geq \gamma>0, \quad \forall x \in \bar{\Omega}_{1}
$$

Logo, por (1.5) e (1.6) obtemos

$$
\frac{\bar{u}(x)}{\varphi_{1}(x)} \geq C_{4}>0, \quad \forall x \in \Omega
$$

onde $C_{4}=\min \left\{C_{2}, \gamma\right\}$. Logo, tomando $0<\varepsilon<C_{2}$ temos que $\varepsilon \varphi_{1} \leq \bar{u}$. Portanto, tomando $\bar{u}=M e$, concluímos que $\varepsilon \varphi_{1} \leq M e$.

Segue-se pelo teorema de sub e supersolução (ver Apêndice $C$, Teorema C.1) que $\left(P_{\lambda}\right)$ tem uma solução $u$, tal que $\varepsilon \varphi_{1} \leq u \leq M e$, sempre que $\lambda \leq \lambda_{0}$, e assim $\Lambda \geq \lambda_{0}$.

Agora, seja $\bar{\lambda}$ tal que

$$
\bar{\lambda} t^{q}+t^{p}>\lambda_{1} t, \quad \forall t \in \mathbb{R}, t>0 .
$$

Isto é possível, já que

$$
\lambda_{1} t-t^{p} \rightarrow-\infty \text { quando } \quad t \rightarrow+\infty
$$

Então existe $t_{0}>1$ tal que

$$
\lambda_{1} t-t^{p} \leq 0, \quad \forall t \geq t_{0} .
$$

Para $t \in\left(1, t_{0}\right]$ temos que existe $C>0$, tal que

$$
\lambda_{1} t-t^{p}<C t^{q} .
$$

Para $t \in(0,1)$ temos

$$
\lambda_{1} t-t^{p}<\lambda_{1} t<\lambda_{1} t^{q} .
$$

Daí, para $\bar{\lambda}=\max \left\{C, \lambda_{1}\right\}$ temos

$$
\lambda_{1} t-t^{p}<\bar{\lambda} t^{q} \quad \text { quando } \quad t>0 .
$$

Se $u \in C^{2}(\Omega), u>0$ é uma solução do problema $\left(P_{\lambda}\right)$ para $\lambda \in(0, \Lambda)$. Assim, multiplicando $(P)_{\lambda}$ por $\varphi_{1}$ e integrando sobre $\Omega$, temos 


$$
\begin{aligned}
-\Delta u & =\lambda u^{q}+u^{p}, \\
\varphi_{1}(-\Delta u) & =\varphi_{1} \lambda u^{q}+\varphi_{1} u^{p} ; \quad 0<q<1<p, \\
\int_{\Omega} \varphi_{1}(-\Delta u) d x & =\int_{\Omega} \lambda \varphi_{1} u^{q} d x+\int_{\Omega} \varphi u^{p} d x .
\end{aligned}
$$

Aplicando agora a Segunda Identidade de Green (ver Apêndice $A$, Teorema A.2) e não esquecendo do fato que $\varphi_{1}$ é uma autofunção positiva associada a $\lambda_{1}$, temos que $\varphi_{1}$ é solução clássica, $\varphi_{1}=0$ sobre $\partial \Omega$ e segue do Lema de Hopf (ver Apêndice $A$, Lema $A .1$ ) que $\frac{\partial \varphi_{1}}{\partial \nu}<0$ sobre $\partial \Omega$, temos que:

$$
\begin{aligned}
\int_{\Omega}\left(\lambda u^{q}+u^{p}\right) \varphi_{1} d x=\int_{\Omega} \varphi_{1}(-\Delta u) d x & =\int_{\Omega} u\left(-\Delta \varphi_{1}\right) d x+\int_{\partial \Omega} u \frac{\partial \varphi_{1}}{\partial \nu} d S-\int_{\partial \Omega} \varphi_{1} \frac{\partial u}{\partial \nu} d S \\
& <\int_{\Omega} u\left(-\Delta \varphi_{1}\right) d x \\
& =\lambda_{1} \int_{\Omega} \varphi_{1} u d x
\end{aligned}
$$

Logo pela escolha de $\bar{\lambda}$ obtemos

$$
\lambda \int_{\Omega} u^{q} \varphi_{1} d x+\int_{\Omega} u^{p} \varphi_{1} d x<\lambda_{1} \int_{\Omega} \varphi_{1} u d x<\bar{\lambda} \int_{\Omega} u^{q} \varphi_{1} d x+\int_{\Omega} u^{p} \varphi_{1} d x .
$$

Assim, $\lambda<\bar{\lambda}$, e dessa forma $\Lambda \leq \bar{\lambda}$.

Portanto,

$$
0<\Lambda<\infty
$$

Lema 1.2. Para todo $0<\lambda<\Lambda$, o problema $\left(P_{\lambda}\right)$ tem uma solução.

Demonstração. Dado $\lambda<\Lambda$, seja $u_{\mu}$ uma solução do problema $\left(P_{\mu}\right)$

$$
\left(P_{\mu}\right)\left\{\begin{aligned}
-\Delta u & =\mu u^{q}+u^{p}, & & x \in \Omega, \\
u & >0, & & x \in \Omega, \\
u & =0, & & x \in \partial \Omega,
\end{aligned}\right.
$$

assim temos que

$$
\left\{\begin{aligned}
-\Delta u_{\mu} & =\mu u_{\mu}^{q}+u_{\mu}^{p}, & & x \in \Omega, \\
u_{\mu} & >0, & & x \in \Omega, \\
u_{\mu} & =0, & & x \in \partial \Omega,
\end{aligned}\right.
$$

considerando $\lambda<\mu<\Lambda$; logo

$$
-\Delta u_{\mu}=\mu u_{\mu}^{q}+u_{\mu}^{p}>\lambda u_{\mu}^{q}+u_{\mu}^{p},
$$

assim, $u_{\mu}$ é supersolução de $\left(P_{\lambda}\right)$. Por outro lado, pela demonstração do Lema 1.1 segue que $\varepsilon \varphi_{1}$ é subsolução de $\left(P_{\lambda}\right)$ para $\varepsilon>0$ suficientemente pequeno. 
Dessa forma, $\varepsilon \varphi_{1}<u_{\mu}$. Logo, pelo Teorema de sub-super solução, existe $u$ solução de $\left(P_{\lambda}\right)$, tal que

$$
\varepsilon \varphi_{1} \leq u \leq u_{\mu}
$$

Portanto, $\left(P_{\lambda}\right)$ possui uma solução.

No que segue, mostraremos que $\left(P_{\lambda}\right)$ possui solução mínima. Para isso, precisamos do seguinte lema:

Lema 1.3. Suponha que $f(t)$ é uma função tal que $t^{-1} f(t)$ é decrescente para $t>0$. Seja $v$ e $w$ satisfazendo,

$$
\left\{\begin{aligned}
-\Delta v & \leq f(v), & & x \in \Omega, \\
v & >0, & & x \in \Omega, \\
v & =0, & & x \in \partial \Omega,
\end{aligned}\right.
$$

$$
\left\{\begin{aligned}
-\Delta w & \geq f(w), & & x \in \Omega, \\
w & >0, & & x \in \Omega, \\
w & =0, & & x \in \partial \Omega .
\end{aligned}\right.
$$

Então $w \geq v e m \Omega$.

Demonstração. A prova foi inspirada pelo Método $I I$ em ([5],p.103). De (1.10) e (1.11) temos

$$
\begin{aligned}
-v \Delta w+w \Delta v & \geq f(w) v-f(v) w \\
& =v w\left(\frac{f(w)}{w}-\frac{f(v)}{v}\right) .
\end{aligned}
$$

Considere agora $\theta(t)$ uma função não decrescente e suave, tal que $\theta(0)=0, \theta(t) \equiv 1$ para $t \geq 1$, e $\theta(t) \equiv 0$ para $t \leq 0$. Assim defina,

$$
\theta_{\varepsilon}(t)=\theta\left(\frac{t}{\varepsilon}\right), \quad \text { onde } \quad \theta_{\varepsilon}(t) \geq 0, \quad \forall t \in \mathbb{R} .
$$

Multiplicando (1.12) por $\theta_{\varepsilon}(v-w)$ e integrando sobre $\Omega$, obtemos

$$
\int_{\Omega}[-v \Delta w+w \Delta v] \theta_{\varepsilon}(v-w) d x \geq \int_{\Omega} v w\left[\frac{f(w)}{w}-\frac{f(v)}{v}\right] \theta_{\varepsilon}(v-w) d x .
$$

Observe que

$$
\begin{aligned}
\int_{\Omega}[-v \Delta w+w \Delta v] \theta_{\varepsilon}(v-w) d x & =\int_{\Omega}\left(-v \Delta w \cdot \theta_{\varepsilon}(v-w)+w \Delta v \cdot \theta_{\varepsilon}(v-w)\right) d x \\
& =\int_{\Omega} \Delta v\left[w \theta_{\varepsilon}(v-w)\right] d x-\int_{\Omega} \Delta w\left[v \theta_{\varepsilon}(v-w)\right] d x
\end{aligned}
$$

Aplicando a Primeira Identidade de Green (ver Apêndice A, Teorema A.2) em (1.14) e o fato que $v=w=0$ em $\partial \Omega ;$ temos

$$
\int_{\Omega}[-v \Delta w+w \Delta v] \theta_{\varepsilon}(v-w) d x=\int_{\Omega} \nabla w \nabla\left[v \theta_{\varepsilon}(v-w)\right] d x-\int_{\Omega} \nabla v \nabla\left[w \theta_{\varepsilon}(v-w)\right] d x
$$


Agora usando a regra da cadeia e agrupando termos, obtemos

$$
\begin{aligned}
\int_{\Omega}[-v \Delta w+w \Delta v] \theta_{\varepsilon}(v-w) d x= & \int_{\Omega} \nabla w\left[\nabla v \cdot \theta_{\varepsilon}(v-w)+v \theta_{\varepsilon}^{\prime}(v-w)(\nabla v-\nabla w)\right] d x \\
& -\int_{\Omega} \nabla v\left[\nabla w \theta_{\varepsilon}(v-w)+w \theta_{\epsilon}^{\prime}(\nabla v-\nabla w)\right] d x \\
= & \int_{\Omega} \nabla w \cdot \nabla v \theta_{\epsilon}(v-w)+\nabla w \cdot v \theta_{\varepsilon}^{\prime}(v-w)(\nabla v-\nabla w) d x \\
& -\int_{\Omega} \nabla v \cdot \nabla w \theta_{\varepsilon}(v-w)+\nabla v \cdot w \theta_{\varepsilon}^{\prime}(v-w)(\nabla v-\nabla w) d x \\
= & \int_{\Omega} v \theta_{\varepsilon}^{\prime}(v-w) \nabla w \cdot(\nabla v-\nabla w) d x \\
& -\int_{\Omega} w \theta_{\varepsilon}^{\prime}(v-w) \nabla v \cdot(\nabla v-\nabla w) d x .
\end{aligned}
$$
que

Somando e subtraindo $\int_{\Omega} v \nabla v(\nabla v-\nabla w) \theta_{\varepsilon}^{\prime}(v-w) d x$ em $(1.15)$, e usando $\gamma_{\varepsilon}(t):=\int_{0}^{t} s \theta_{\varepsilon}^{\prime}(s) d s$, temos

$$
\begin{aligned}
\int_{\Omega}[-v \Delta w+w \Delta v] \theta_{\varepsilon}(v-w) d x & =\int_{\Omega} v \theta_{\varepsilon}^{\prime}(v-w) \nabla w \cdot(\nabla v-\nabla w) d x-\int_{\Omega} w \theta_{\varepsilon}^{\prime}(v-w) \nabla v \cdot(\nabla v-\nabla w) d x \\
& +\int_{\Omega} v \nabla v(\nabla v-\nabla w) \theta_{\epsilon}^{\prime}(v-w) d x-\int_{\Omega} v \nabla v(\nabla v-\nabla w) \theta_{\varepsilon}^{\prime}(v-w) d x \\
= & \int_{\Omega} v \theta_{\varepsilon}^{\prime}(v-w)(\nabla w-\nabla v)(\nabla v-\nabla w) d x \\
& +\int_{\Omega}(v-w) \theta_{\varepsilon}^{\prime}(v-w) \nabla v(\nabla v-\nabla w) d x \\
\leq & \int_{\Omega}(v-w) \theta_{\varepsilon}^{\prime}(v-w) \nabla v(\nabla v-\nabla w) d x \\
= & \int_{\Omega} \nabla v \cdot \nabla\left[\gamma_{\varepsilon}(v-w)\right] d x .
\end{aligned}
$$

Aplicando novamente a Primeira Identidade de Green em (1.16), segue que

$$
\int_{\Omega} \nabla v \cdot \nabla\left[\gamma_{\varepsilon}(v-w)\right] d x=-\int_{\Omega} \Delta v \cdot \gamma_{\varepsilon}(v-w) d x .
$$

Agora, uma vez que

$$
0 \leq \gamma_{\varepsilon}(t) \leq \varepsilon, \quad \forall t \in \mathbb{R}
$$

segue que

$$
\int_{\Omega}[-v \Delta w+w \Delta v] \theta_{\varepsilon}(v-w) d x \leq \varepsilon
$$

De fato, de (1.16) e (1.17) temos que

$$
\int_{\Omega}[-v \nabla w+w \nabla v] \theta_{\varepsilon}(v-w) d x \leq \int_{\Omega}-\Delta v \gamma_{\varepsilon}(v-w) d x
$$

e sabe-se que $-\Delta v \leq f(v)$, e $\gamma_{\varepsilon} \leq \varepsilon$, para todo $t \in \mathbb{R}$; escolhendo um $\varepsilon>0$ suficientemente pequeno, temos que 


$$
\int_{\Omega}[-v \Delta w+w \Delta v] \theta_{\varepsilon}(v-w) d x \leq \varepsilon
$$

Agora, aplicando a desigualdade (1.18) em (1.13) obtemos,

$$
\int_{\Omega} v w\left[\frac{f(w)}{w}-\frac{f(v)}{v}\right] \theta_{\varepsilon}(v-w) d x \leq \varepsilon .
$$

Fazendo, $\varepsilon \rightarrow 0$, temos

$$
\int_{[v>w]} v w\left[\frac{f(w)}{w}-\frac{f(v)}{v}\right] d x \leq 0 .
$$

Por outro lado, $\frac{f(v)}{v}<\frac{f(w)}{w}$ sob $[v>w]$, isto é meas $[v>w]=0$; então $v \leq w$.

Isto completa a prova do lema.

Lema 1.4. Para todo $0<\lambda<\Lambda$, o problema $\left(P_{\lambda}\right)$ tem uma solução mínima $u_{\lambda}$.

Demonstração. Seja $v_{\lambda}$ a única solução positiva de

$$
\left\{\begin{aligned}
-\Delta v & =\lambda v^{q}, & & x \in \Omega, \\
v & =0, & & x \in \partial \Omega .
\end{aligned}\right.
$$

Sabe-se que existe uma solução $u>0$ de $\left(P_{\lambda}\right)$ para todo $\lambda \in(0, \Lambda)$ (Pelo Lema 1.2). Usaremos o símbolo $u_{\lambda}$, tal $\lambda \in(0, \Lambda)$ para denotar a solução mínima de $\left(P_{\lambda}\right)$.

Agora, como

$$
\left\{\begin{aligned}
-\Delta u & =\lambda u^{q}+u^{p} \geq \lambda u^{q}, & & x \in \Omega, \\
u & >0, & & x \in \Omega, \\
u & =0, & & x \in \partial \Omega .
\end{aligned}\right.
$$

Usando o Lema $1.3 \operatorname{com} w=u$ e $v=v_{\lambda}$ e definindo $f_{\lambda}(u):=\lambda u^{q}+u^{p}$, temos que

$$
\left\{\begin{aligned}
-\Delta v_{\lambda} & =\lambda v_{\lambda}^{q} \leq \lambda v_{\lambda}^{q}+v_{\lambda}^{p}=f_{\lambda}\left(v_{\lambda}\right), & & x \in \Omega, \\
v_{\lambda} & >0, & & x \in \Omega, \\
v_{\lambda} & =0, & & x \in \partial \Omega
\end{aligned}\right.
$$

e

$$
\left\{\begin{aligned}
-\Delta u & =\lambda u^{q}+u^{p}=f_{\lambda}(u), & & x \in \Omega, \\
u & >0, & & x \in \Omega, \\
u & =0, & & x \in \partial \Omega,
\end{aligned}\right.
$$

assim temos que

$$
u \geq v_{\lambda}
$$

para qualquer solução $u$ de $\left(P_{\lambda}\right)$. Logo, é claro que $v_{\lambda}$ é subsolução de $\left(P_{\lambda}\right)$.

Considere agora a iteração monótona

$$
\left\{\begin{aligned}
-\Delta u_{n+1} & =\lambda u_{n}^{q}+u_{n}^{p}, & & x \in \Omega, \\
u_{n+1} & =0, & & x \in \partial \Omega,
\end{aligned}\right.
$$


onde $u_{0}=v_{\lambda}$, satisfazendo, $u_{n} \uparrow u_{\lambda}$, com $u_{\lambda}$ solução de $\left(P_{\lambda}\right)$.

Afirmação 1.1. $u_{\lambda}$ é solução mínima de $\left(P_{\lambda}\right)$.

De fato, se $u$ é solução de $\left(P_{\lambda}\right)$, por (1.19) temos que $u \geq v_{\lambda}$, assim $u$ é supersolução de $\left(P_{\lambda}\right)$. Então $u_{n} \leq u, \forall n \in \mathbb{N}$. Para verificar isso usaremos indução, isto é, mostraremos que:

$$
v_{\lambda}=u_{0} \leq u_{1} \leq \cdots \leq u_{n} \leq \cdots \leq u
$$

De fato, primeiramente vamos mostrar que $v_{\lambda}=u_{0} \leq u_{1}$. Como $v_{\lambda}$ é uma subsolução de $\left(P_{\lambda}\right)$, temos que

$$
\left\{\begin{aligned}
-\Delta v_{\lambda} & \leq \lambda v_{\lambda}^{q}+v_{\lambda}^{p}, & & x \in \Omega, \\
v_{\lambda} & \leq 0, & & x \in \partial \Omega .
\end{aligned}\right.
$$

Além disso $u_{1}$ é solução de (1.20) logo,

$$
\left\{\begin{aligned}
-\Delta u_{1} & =\lambda v_{\lambda}^{q}+v_{\lambda}^{p}, & & x \in \Omega, \\
u_{1} & =0, & & x \in \partial \Omega,
\end{aligned}\right.
$$

dessa forma

$$
\left\{\begin{aligned}
-\Delta v_{\lambda} & \leq-\Delta u_{1}, & & x \in \Omega \\
v_{\lambda} & \leq u_{1}, & & x \in \partial \Omega
\end{aligned}\right.
$$

assim

$$
\left\{\begin{array}{rlrl}
-\Delta\left(u_{1}-v_{\lambda}\right) & \geq 0, & & x \in \Omega \\
u_{1}-v_{\lambda} \geq 0, & & x \in \partial \Omega .
\end{array}\right.
$$

Logo, pelo Princípio do Máximo (ver Apêndice $A$, Teorema A.4), temos que $u_{1}-v_{\lambda} \geq 0$ em $\Omega$, ou seja $u_{1} \geq v_{\lambda}$ em $\Omega$. Agora, vamos mostrar que $u_{1} \leq u$ em $\Omega$. Como $u$ é supersolução de $\left(P_{\lambda}\right)$ temos

$$
\left\{\begin{aligned}
-\Delta u & \geq \lambda u^{q}+u^{p}, & & x \in \Omega, \\
u & \geq 0, & & x \in \partial \Omega
\end{aligned}\right.
$$

e usando que $f_{\lambda}(\cdot)$ é crescente, temos que $f_{\lambda}\left(v_{\lambda}\right) \leq f_{\lambda}(u)$ e de $(1.20)$, assim

$$
\left\{\begin{aligned}
-\Delta u_{1} & =\lambda u_{0}^{q}+u_{0}^{p}=\lambda v_{\lambda}^{q}+v_{\lambda}^{p} \leq \lambda u^{q}+u^{p}, & & x \in \Omega \\
u_{1} & =0, & & x \in \partial \Omega
\end{aligned}\right.
$$

então,

$$
\left\{\begin{aligned}
-\Delta u_{1} & \leq f_{\lambda}(u) \leq-\Delta u, & & x \in \Omega \\
u_{1} & \leq u, & & x \in \partial \Omega,
\end{aligned}\right.
$$

dessa forma segue que

$$
\left\{\begin{aligned}
-\Delta\left(u-u_{1}\right) & \geq 0, & & x \in \Omega, \\
u-u_{1} & \geq 0, & & x \in \partial \Omega .
\end{aligned}\right.
$$

Portanto, pelo Princípio do Máximo temos que $u_{1} \leq u$ em $\Omega$.

Agora, suponhamos por indução que vale, $v_{\lambda}=u_{0} \leq u_{1} \leq \cdots \leq u_{n} \leq u$, e mostraremos que $v_{\lambda}=u_{0} \leq u_{n+1} \leq u$. 
Por (1.20) considere as sequências $u_{n}$ e $u_{n+1}$ definidas por

$$
\left\{\begin{aligned}
-\Delta u_{n} & =\lambda u_{n-1}^{q}+u_{n-1}^{p}, & & x \in \Omega, \\
u_{n} & =0, & & x \in \partial \Omega
\end{aligned}\right.
$$

e

$$
\left\{\begin{aligned}
-\Delta u_{n+1} & =\lambda u_{n}^{q}+u_{n}^{p}, & & x \in \Omega, \\
u_{n+1} & =0, & & x \in \partial \Omega,
\end{aligned}\right.
$$

subtraindo (1.21) de (1.22) temos

$$
\left\{\begin{aligned}
-\Delta\left(u_{n+1}-u_{n}\right) & =\lambda u_{n}^{q}+u_{n}^{p}-\left(\lambda u_{n-1}^{q}+u_{n-1}^{p}\right), & & x \in \Omega, \\
u_{n+1}-u_{n} & =0, & & x \in \partial \Omega,
\end{aligned}\right.
$$

contudo, como $f_{\lambda}(\cdot)$ é crescente, temos que

$$
\lambda u_{n}^{q}+u_{n}^{p}-\left(\lambda u_{n-1}^{q}+u_{n-1}^{p}\right) \geq 0
$$

Portanto, pelo Princípio do Máximo $u_{n+1}-u_{n} \geq 0$, em $\Omega$, isto é, $u_{n+1} \geq u_{n}$, em $\Omega$. Análogo a demonstração de $u_{1} \leq u$, em $\Omega$, verifica-se que $u_{n+1} \leq u$, em $\Omega, \forall n \in \mathbb{N}$. Mas como $u_{n} \uparrow u_{\lambda}$, temos que $u_{\lambda} \leq u$, demonstrando assim a afirmação. Concluímos assim que $u_{\lambda}$ é solução mínima.

Observação 1.1. É importante ressaltar que da teoria espectral para o operador dado por $-\Delta-a(x)$, onde $a(x):=\lambda q u^{q-1}+p u^{p-1}$, pode ser transferida para $H$, ainda se $a(x)=+\infty$ em $\partial \Omega$. Onde, observamos que

$$
\int_{\Omega} a \phi^{2} d x \leq C_{1}\|\phi\|^{2}, \quad \forall \phi \in H .
$$

Para verificarmos este fato, note que

$$
\int_{\Omega} u^{q-1} \phi^{2} d x=\int_{\Omega} u^{q}\left(\frac{1}{u} \phi\right) \phi d x \leq\|u\|_{\infty}^{q} \cdot\left\|\frac{\phi}{\delta}\right\|_{2} \cdot\|\phi\|_{2}
$$

onde $\delta(x)=\operatorname{dist}(x, \partial \Omega)$. Pela desigualdade de Hardy (ver Apêndice A, Teorema A.13), temos

$$
\left\|\frac{\phi}{\delta}\right\|_{2} \leq C_{2}\|\nabla \phi\|_{2}=C_{2}\|\phi\|, \quad \forall \phi \in H
$$

Segue que

$$
\int_{\Omega} u^{q-1} \phi^{2} d x \leq C_{3}\|\phi\|^{2}
$$

De modo análogo, mostra-se que

$$
\int_{\Omega} u^{p-1} \phi^{2} d x \leq C_{4}\|\phi\|^{2}
$$

Portanto, de (1.25) e (1.26), temos a desigualdade (1.24).

Mais ainda, a aplicação $\phi \mapsto \int_{\Omega} u \phi^{2} d x$ é sequencialmente contínua para a topologia fraca de $H^{1,2}$, 
pois

$$
\left|\int_{\Omega} u^{q-1}\left(\phi_{n}^{2}-\phi^{2}\right) d x\right| \leq\|u\|_{\infty}^{q}\left\|\frac{\phi_{n}+\phi}{\delta}\right\|_{2}\left\|\phi_{n}-\phi\right\|_{2} \rightarrow 0
$$

Lema 1.5. Sejam $\psi \leq \Psi$ uma subsolução e uma supersolução respetivamente de $\left(P_{\lambda}\right)$, e suponha que $\psi$ não é solução. Seja u uma solução mínima, tal que $\psi \leq u \leq \Psi$. Então $\nu_{1}:=\lambda_{1}[-\Delta-a(x)] \geq 0$, onde $a=a(x)=\lambda q u^{q-1}+p u^{p-1}$ e $\lambda_{1}[-\Delta-a(x)]$ denota o primeiro autovalor de $-\Delta-a(x)$, com condição de Dirichlet sobre a fronteira.

Demonstração. Suponha por contradição que $\nu_{1}<0$ e denotaremos por $\bar{\phi}>0$ a correspondente autofunção, assim:

$$
\left\{\begin{aligned}
-\Delta \bar{\phi}-a \bar{\phi} & =\nu_{1} \bar{\phi}, & & x \in \Omega \\
\bar{\phi} & =0, & & x \in \partial \Omega .
\end{aligned}\right.
$$

Afirmamos que $u-\alpha \bar{\phi}$ é supersolução de $\left(P_{\lambda}\right)$ para $\alpha>0$, suficientemente pequeno.

De fato,

$$
\begin{aligned}
-\Delta(u & -\alpha \bar{\phi})-\left[\lambda(u-\alpha \bar{\phi})^{q}+(u-\alpha \bar{\phi})^{p}\right] \\
& =-\Delta u+\alpha \Delta \bar{\phi}-\left[\lambda(u-\alpha \bar{\phi})^{q}+(u-\alpha \bar{\phi})^{p}\right] \\
& =\lambda u^{q}+u^{p}+\alpha\left(-\nu_{1} \bar{\phi}-a \bar{\phi}\right)-\left[\lambda(u-\alpha \bar{\phi})^{q}+(u-\alpha \bar{\phi})^{p}\right] \\
& =\lambda u^{1}+u^{p}-\alpha \nu_{1} \bar{\phi}-\alpha a \bar{\phi}-\left[\lambda(u-\alpha \bar{\phi})^{q}+(u-\alpha \bar{\phi})^{p}\right] \\
& =\lambda u^{q}+u^{p}-\alpha \nu_{1} \bar{\phi}-\alpha\left(\lambda q u^{q-1}+p u^{p-1}\right) \bar{\phi}-\left[\lambda(u-\alpha \bar{\phi})^{q}+(u-\alpha \bar{\phi})^{p}\right] .
\end{aligned}
$$

Uma vez que $t \mapsto t^{q}$ é côncava, temos que

$$
(u-\alpha \bar{\phi})^{q} \leq u^{q}-\alpha q u^{q-1} \bar{\phi}
$$

Assim,

$$
\begin{aligned}
-\Delta & (u-\alpha \bar{\phi})-\left[\lambda(u-\alpha \bar{\phi})^{q}+(u-\alpha \bar{\phi})^{p}\right] \\
& =\lambda u^{q}+u^{p}-\alpha \nu_{1} \bar{\phi}-\alpha\left(\lambda q u^{q-1}+p u^{p-1}\right) \bar{\phi}-\left[\lambda(u-\alpha \bar{\phi})^{q}+(u-\alpha \bar{\phi})^{p}\right] \\
& \geq \lambda u^{q}+u^{p}-\alpha \nu_{1} \bar{\phi}-\alpha \lambda q u^{q-1} \bar{\phi}-\alpha p u^{p-1} \bar{\phi}-\lambda u^{q-1}+\alpha q u^{q-1} \bar{\phi}-(u-\alpha \bar{\phi})^{p} \\
& =u^{p}-\alpha \nu_{1} \bar{\phi}-\alpha p u^{p-1} \bar{\phi}-(u-\alpha \bar{\phi})^{p} \\
& =-\alpha \nu_{1} \bar{\phi}+o_{n}(\alpha \bar{\phi})>0,
\end{aligned}
$$

para $\alpha>0$ pequeno, pois $\nu_{1}<0$ e $\bar{\phi}>0$. Portanto, $u-\alpha \bar{\phi}$ é a supersolução de $\left(P_{\lambda}\right)$. Mais ainda, como $\psi$ não é solução, logo $u>\psi$ e, tomando $\alpha$ suficientemente pequeno, podemos também supor que $u-\alpha \bar{\phi} \geq \psi$. Logo $\left(P_{\lambda}\right)$ tem uma solução $\tilde{u}, \operatorname{com} \psi \leq \tilde{u} \leq u-\alpha \bar{\phi}$, uma contradição pois $u$ é mínima. Isto prova o lema.

Observamos que $\lambda_{1}[-\Delta-a(x)] \geq 0$ se, e somente se,

$$
\int_{\Omega}\left(|\nabla \phi|^{2}-a \phi^{2}\right) d x \geq 0, \quad \forall \phi \in H
$$

Assim, usando o Lema 1.5 com $u_{\lambda}$ solução mínima, temos em particular que (1.27) com $a=a_{\lambda}=$ 
$\lambda q u_{\lambda}^{q-1}+p u_{\lambda}^{p-1}$, é satisfeita, isto é

$$
\int_{\Omega}\left(|\nabla \phi|^{2}-a_{\lambda} \phi^{2}\right) d x \geq 0, \quad \forall \phi \in H
$$

Agora temos todas as ferramentas necessárias para demonstrarmos o Teorema 1.1.

\section{Demonstração do Teorema 1.1}

Item 1. A partir dos Lemas 1.1, 1.2 e 1.4 segue que $\left(P_{\lambda}\right)$ tem solução mínima $u_{\lambda}$ para todo $\lambda \in(0, \Lambda)$. Note que

$$
I_{\lambda}\left(u_{\lambda}\right)=\frac{1}{2}\left\|u_{\lambda}\right\|^{2}-\frac{\lambda}{q+1}\left\|u_{\lambda}\right\|_{q+1}^{q+1}-\frac{1}{p+1}\left\|u_{\lambda}\right\|_{p+1}^{p+1} .
$$

Como $u_{\lambda}$ é solução de $\left(P_{\lambda}\right)$, temos que

$$
I_{\lambda}^{\prime}\left(u_{\lambda}\right) u_{\lambda}=0
$$

assim, obtemos

$$
\left\|u_{\lambda}\right\|^{2}=\lambda\left\|u_{\lambda}\right\|_{q+1}^{q+1}+\left\|u_{\lambda}\right\|_{p+1}^{p+1} .
$$

Pelo Lema 1.5 e a Observação 1.1, em particular da expresão (1.28) com $\phi=u_{\lambda}$, temos que

$$
\int_{\Omega}\left(\left|\nabla u_{\lambda}\right|^{2}-\left(\lambda q u_{\lambda}^{q-1}+p u_{\lambda}^{p-1}\right) u_{\lambda}^{2}\right) d x \geq 0
$$

logo, obtemos

$$
\left\|u_{\lambda}\right\|^{2}-\lambda q\left\|u_{\lambda}\right\|_{q+1}^{q+1}-p\left\|u_{\lambda}\right\|_{p+1}^{p+1} \geq 0
$$

Agora, por outro lado temos que $I_{\lambda}\left(u_{\lambda}\right)<0$, pois

$$
\begin{aligned}
I_{\lambda}\left(u_{\lambda}\right) & =\frac{1}{2}\left\|u_{\lambda}\right\|^{2}-\frac{\lambda}{q+1}\left\|_{q+1}^{q+1}-\frac{1}{p+1}\right\| u_{\lambda} \|_{p+1}^{p+1} \\
& =\frac{1}{2}\left[\lambda\left\|u_{\lambda}\right\|_{q+1}^{q+1}+\left\|u_{\lambda}\right\|_{p+1}^{p+1}\right]-\frac{\lambda}{q+1}\left\|u_{\lambda}\right\|_{q+1}^{q+1}-\frac{1}{p+1}\left\|u_{\lambda}\right\|_{p+1}^{p+1} \\
& =\lambda\left\|u_{\lambda}\right\|_{q+1}^{q+1}\left[\frac{1}{2}-\frac{1}{q+1}\right]+\left\|u_{\lambda}\right\|_{p+1}^{p+1}\left[\frac{1}{2}-\frac{1}{p+1}\right]
\end{aligned}
$$

entretanto,

$$
0<q<1<p \quad \text { e } \quad 1<q+1<2<p+1
$$

dessa forma:

$$
\begin{aligned}
& q+1<2 \quad \log 0 \quad \frac{1}{2}<\frac{1}{q+1} \quad \text { assim } \quad \frac{1}{2}-\frac{1}{q+1}<0 \\
& 2<p+1 \quad \operatorname{logo} \quad \frac{1}{p+1}<\frac{1}{2} \quad \text { assim } \quad \frac{1}{p+1}-\frac{1}{2}>0 \quad \text { portanto } \quad \frac{1}{2}-\frac{1}{p+1}<0 .
\end{aligned}
$$

Concluímos que $I_{\lambda}\left(u_{\lambda}\right)<0$. 
Para completar a prova do Item 1, falta mostrarmos que

$$
u_{\lambda}<u_{\bar{\lambda}} \text { quando } \quad \lambda<\bar{\lambda} \text {. }
$$

De fato, se $\lambda<\bar{\lambda}$ então $u_{\bar{\lambda}}$ é supersolução de $\left(P_{\lambda}\right)$. Desde que, $\varepsilon>0$ seja suficientemente pequeno $\varepsilon \phi_{1}$ é subsolução de $\left(P_{\lambda}\right)$ e $\varepsilon \phi_{1}<u_{\bar{\lambda}}$, então $\left(P_{\lambda}\right)$ possui uma solução $v$ com

$$
\varepsilon \phi_{1} \leq v \leq u_{\bar{\lambda}}
$$

Uma vez que $u_{\lambda}$ é solução mínima de $\left(P_{\lambda}\right)$, temos que $u_{\lambda} \leq v \leq u_{\bar{\lambda}}$. A desigualdade estrita segue pelo Princípio do Máximo Forte, pois $u_{\lambda}$ não é identicamente igual a $u_{\bar{\lambda}}$. Isto completa a prova do Item 1.

Item 2. Seja $\left(\lambda_{n}\right)$ uma sequência tal que $\lambda_{n} \uparrow \Lambda$. Uma vez que $u_{n}=u_{\lambda_{n}}$, pelo Item 1 , temos que $u_{\lambda_{n}}$ é solução mínima do problema $\left(P_{\lambda}\right)$ e $I_{\lambda_{n}}\left(u_{n}\right)<0$. Logo temos que, existe $C>0$ tal que

$$
\left\|\nabla u_{n}\right\|^{2} \leq C \quad \text { e } \quad\left\|u_{n}\right\|_{p+1}^{p+1} \leq C
$$

$\operatorname{assim}\left(u_{n}\right)$ é uma sequência limitada em $H$, logo existe $u^{*} \in H$ tal que $u_{n} \rightarrow u^{*}$ em quase todo ponto de $\Omega$. Além disso, temos que $\left(u_{n}\right) \subset L^{p+1}$ é limitada, assim $u_{n} \rightarrow \bar{u}$ fortemente em $L^{p+1}$. (ver Apêndice $A$, Proposição A.1).

Dessa forma, pela unicidade do limite e do fato que convergência forte implica convergência fraca, temos que $u^{*}=\bar{u}$. Portanto, $u^{*} \in H \cap L^{p+1}$ é solução fraca de $\left(P_{\lambda}\right)$ para $\lambda=\Lambda$.

Item 3. Lembre que

$$
\Lambda=\sup \left\{\lambda>0:\left(P_{\lambda}\right) \text { tem solução }\right\}
$$

Logo, o Item 3 segue pela definição de $\Lambda$.

Observação 1.2. Desde que $M(\lambda) \rightarrow 0$ quando $\lambda \rightarrow 0$ (veja a prova do Lema 1.1), segue $\left\|u_{\lambda}\right\|_{\infty} \rightarrow 0$ quando $\lambda \rightarrow 0$.

O próximo resultado, estuda o comportamento de $\left\|u_{\lambda}\right\|_{\infty}$ quando $\lambda \rightarrow 0$, onde $u_{\lambda}$ é solução de $\left(P_{\lambda}\right)$.

Teorema 1.2. Existe $A>0$, tal que para todo $\lambda \in(0, \Lambda)$ o problema $\left(P_{\lambda}\right)$ tem no máximo uma solução $u$, tal que $\|u\|_{\infty} \leq A$.

Na sequência apresentaremos um lema que será importante para a prova do Teorema 1.2.

Lema 1.6. Suponha que z denota a única solução mínima satisfazendo

$$
\left\{\begin{array}{rlrl}
-\Delta z & =z^{q}, & & x \in \Omega, \\
z>0, & & x \in \Omega, \\
z & =0, & & x \in \partial \Omega .
\end{array}\right.
$$

Então existe $\beta>0$ tal que 


$$
\int_{\Omega}\left[|\nabla \phi|^{2}-q z^{q-1} \phi^{2}\right] d x \geq \beta\|\phi\|_{2}^{2}, \quad \forall \phi \in H
$$

Demonstração. Temos que o funcional associado ao problema (1.30) é

$$
I(u)=\frac{1}{2}\|u\|^{2}-\frac{1}{q+1}\|u\|_{q+1}^{q+1}
$$

$\log \mathrm{O}$

$$
z=\min I(u)=\min \left\{\frac{1}{2}\|u\|^{2}-\frac{1}{q+1} \|_{q+1}^{q+1}, \quad u \in H\right\} .
$$

Assim, por (1.28) com $a=q z^{q-1}$ temos que

$$
\int_{\Omega}\left[|\nabla \phi|^{2}-q z^{q-1} \phi^{2}\right] d x \geq 0, \quad \forall \phi \in H,
$$

isto é $\lambda_{1}\left[-\Delta-q z^{q-1}\right] \geq 0$. Suponha que $\lambda_{1}\left[-\Delta-q z^{q-1}\right]=0$. Logo existe $\phi \in H, \phi>0$ tal que

$$
-\Delta \phi-q z^{q-1} \phi=0
$$

e então

$$
\int_{\Omega} \nabla \phi \cdot \nabla z d x=q \int_{\Omega} z^{q} \phi d x
$$

Por outro lado, usando (1.30) obtemos que

$$
\int_{\Omega} \nabla \phi \cdot \nabla z d x=\int_{\Omega} z^{q} \phi d x
$$

A última expresão contradiz (1.32), pois $q<1$. Então $\lambda_{1}\left[-\Delta-q z^{q-1}\right]>0$, isto é (1.31) é verdadeiro.

Agora estamos prontos para demonstrar o Teorema 1.2.

\section{Demonstração do Teorema 1.2}

Considere $A>0$ tal que

$$
p A^{p-1}<\beta
$$

onde $\beta$ é o valor encontrado no Lema 1.6. Além disso, no Lema 1.1 mostramos que para todo $\lambda \in(0, \Lambda)$, $\left(P_{\lambda}\right)$ tem no máximo uma solução $u$, a qual pela Observação 1.2 satisfaz

$$
\|u\|_{\infty} \leq A
$$

Suponha por contradição que $\left(P_{\lambda}\right)$ tem uma segunda solução $w=u_{\lambda}+v$, tal que

$$
\|w\|_{\infty} \leq A
$$

Note que como $u_{\lambda}$ é solução mínima de $\left(P_{\lambda}\right)$, então $v>0$. Agora tomando $\zeta(x)=\lambda^{\frac{1}{1-q}} \cdot z(x)$, onde $z$ é a mesma do Lema 1.6 obtemos 


$$
\begin{aligned}
-\Delta \zeta & =-\Delta\left[\lambda^{\frac{1}{1-q}} \cdot z\right]=\lambda^{\frac{1}{1-q}}(-\Delta z) \\
& =\lambda^{\frac{1}{1-q}} \cdot z^{q} \\
& =\lambda^{\frac{1}{1-q}} \cdot\left(\zeta \lambda^{\frac{-1}{1-q}}\right)^{q} \\
& =\lambda^{\frac{1}{1-q}} \zeta^{q} \lambda^{\frac{-q}{1-q}}=\lambda \cdot \zeta^{q},
\end{aligned}
$$

então temos que, $-\Delta \zeta=\lambda \zeta^{q}$.

Mais ainda, temos que

$$
-\Delta u_{\lambda}=\lambda u_{\lambda}^{q}+u_{\lambda}^{p} \geq \lambda u_{\lambda}^{q}
$$

e usando o Lema 1.3, considerando $f(t)=\lambda t^{q}, v=\zeta$ e $w=u_{\lambda}$, segue que

$$
u_{\lambda} \geq \zeta=\lambda^{\frac{1}{1-q}} \cdot z
$$

Por outro lado, como $w=u_{\lambda}+v$ é solução de $\left(P_{\lambda}\right)$ temos $-\Delta w=\lambda w^{q}+w^{p}$, isto é,

$$
-\Delta\left(u_{\lambda}+v\right)=\lambda\left(u_{\lambda}+v\right)^{q}+\left(u_{\lambda}+v\right)^{p} .
$$

Pela concavidade da função $f(t)=t^{q}, 0<q<1$; temos que

$$
\lambda\left(u_{\lambda}+v\right)^{q} \leq \lambda u_{\lambda}^{q}+\lambda q u_{\lambda}^{q-1} v
$$

e assim,

$$
\begin{aligned}
-\Delta\left(u_{\lambda}+v\right) & =\lambda\left(u_{\lambda}+v\right)^{q}+\left(u_{\lambda}+v\right)^{p} \\
-\Delta u_{\lambda}-\Delta v & =\lambda\left(u_{\lambda}+v\right)^{q}+\left(u_{\lambda}+v\right)^{p} \\
-\Delta v & =\lambda\left(u_{\lambda}+v\right)^{q}+\left(u_{\lambda}+v\right)^{p}+\Delta u_{\lambda} \\
-\Delta v & =\lambda\left(u_{\lambda}+v\right)^{q}+\left(u_{\lambda}+v\right)^{p}-\lambda u_{\lambda}^{q}-u_{\lambda}^{p} \\
& \leq \lambda u_{\lambda}^{q}+\lambda q u_{\lambda}^{q-1} v+\left(u_{\lambda}+v\right)^{p}-\lambda u_{\lambda}^{q}-u_{\lambda}^{p} \\
& =\lambda q u_{\lambda}^{q-1} v+\left(u_{\lambda}+v\right)^{p}-u_{\lambda}^{p}
\end{aligned}
$$

provando que,

$$
-\Delta v \leq \lambda q u_{\lambda}^{q-1} v+\left(u_{\lambda}+v\right)^{p}-u_{\lambda}^{p} .
$$

Mais ainda de (1.35) temos

$$
u_{\lambda}^{q-1} \leq\left(\lambda^{\frac{1}{1-q}} z\right)^{q-1}=\lambda^{-1} z^{q-1} .
$$

Assim de (1.36) e (1.37) concluímos que:

$$
\begin{aligned}
-\Delta v & \leq \lambda q\left(\lambda^{-1} z^{q-1}\right) v+\left(u_{\lambda}+v\right)^{p}-u_{\lambda}^{p} \\
& =q z^{q-1} v+\left(u_{\lambda}+v\right)^{p}-u_{\lambda}^{p} .
\end{aligned}
$$

Além disso, como $u_{\lambda}+v=w \leq\|w\|_{\infty} \leq A$, temos que

$$
\left(u_{\lambda}+v\right)^{p}-u_{\lambda}^{p} \leq p A^{p-1} v
$$


e assim,

$$
-\Delta v-q z^{q-1} v \leq\left(u_{\lambda}+v\right)^{p}-u_{\lambda}^{p} \leq p A^{p-1} v .
$$

Agora, multiplicando esta desigualdade por $v$ e integrando sobre $\Omega$, sabendo que $v=0$ em $\partial \Omega$, pois $v \in H$. Temos,

$$
\begin{aligned}
-v \Delta v-q z^{q-1} v^{2} & \leq p A^{p-1} v^{2} \\
\int_{\Omega}-v \Delta v-q z^{q-1} v^{2} d x & \leq \int_{\Omega} p A^{p-1} v^{2} d x \\
\int_{\Omega}|\nabla v|^{2} d x-\int_{\partial \Omega} \frac{\partial v}{\partial \eta} d S-\int_{\Omega} q z^{q-1} v^{2} d x & \leq \int_{\Omega} p A^{p-1} v^{2} d x \\
\int_{\Omega}\left(|\nabla v|^{2}-q z^{q-1} v^{2}\right) d x & \leq p A^{p-1} \int_{\Omega} v^{2} d x .
\end{aligned}
$$

Aplicando o resultado do Lema 1.6, $\operatorname{com} \phi=v$ temos que existe $\beta>0$ tal que

$$
\begin{aligned}
\beta\|v\|_{2}^{2} & =\beta \int_{\Omega}|v|^{2} d x \\
& \leq \int_{\Omega}\left(|\nabla v|^{2}-q z^{q-1} v^{2}\right) d x \\
& \leq p A^{p-1} \int_{\Omega} v^{2} d x
\end{aligned}
$$

assim,

$$
\beta \int_{\Omega}|v|^{2} d x \leq p A^{p-1} \int_{\Omega}|v|^{2} d x
$$

mas como $p A^{p-1}<\beta$, segue que $v=0$, o que é uma contradição pois $v>0$. Concluindo assim a prova do Teorema 1.2.

Observação 1.3. O comportamento de $u_{\lambda}$ perto de $\lambda=0$ é de tal forma que $u_{\lambda} \simeq \lambda^{\frac{1}{1-q}} z$.

\subsection{Existência de uma segunda solucão por argumentos variacio- nais}

Nesta seção, consideraremos que

$$
q<1<p \leq \frac{N+2}{N-2}=2^{*}-1
$$

Em particular, considere

$$
f_{\lambda}(s)=\left\{\begin{aligned}
\lambda s^{q}+s^{p}, & s \geq 0, \\
0, & s<0
\end{aligned} \quad \text { e } \quad F_{\lambda}(u)=\int_{0}^{u} f_{\lambda}(s) d s .\right.
$$

Assim, definimos o funcional $\quad \bar{I}_{\lambda}: H \rightarrow \mathbb{R}$ como sendo

$$
\bar{I}_{\lambda}(u)=\frac{1}{2}\|u\|^{2}-\int_{\Omega} F_{\lambda}(u) d x .
$$

Claramente, temos que $I_{\lambda}(u)=\bar{I}_{\lambda}(u)$ quando $u>0$. Mais ainda, sabemos que pontos críticos de $\bar{I}_{\lambda}$ correspondem a soluções de $\left(P_{\lambda}\right)$. Na seção anterior (no Lema 1.5 e Observação 1.1) garantem a existência 
de uma solução mínima $u_{\lambda}$ de $\left(P_{\lambda}\right)$ tal que $\nu_{1}:=\lambda_{1}\left[-\Delta-a_{\lambda}\right] \geq 0$. Se $\nu_{1}>0$ a solução é um mínimo local de $\bar{I}_{\lambda}$, mas se $\nu_{1}=0$ isto não é necessariamente o caso.

Para nosso objetivo que é encontrarmos uma segunda solução por métodos variacionais é essencial ter uma primeira solução a qual é também um mínimo local.

O resultado importante nesta seção é o seguinte,

Teorema 1.3. Seja $0<q<1<p \leq \frac{N+2}{N-2}$. Então para todo $\lambda \in(0, \Lambda)$ o problema $\left(P_{\lambda}\right)$ tem uma segunda solução $v_{\lambda}>u_{\lambda}$.

Para fazermos a demonstração desse Teorema, mostraremos alguns resultados preliminares que serão úteis para a demonstração do Teorema.

Lema 1.7. Para todo $\lambda \in(0, \Lambda)$ o problema $\left(P_{\lambda}\right)$ tem uma solução $u$, que é um mínimo local de $\bar{I}_{\lambda}$ na topologia $C^{1}$.

Demonstração. Fixe $\lambda \in\left(\lambda_{1}, \Lambda\right)$. Escolha $\lambda_{1}<\lambda<\lambda_{2}<\Lambda$, e considere as soluções mínimas $u_{1}:=$ $u_{\lambda_{1}}$ e $u_{2}:=u_{\lambda_{2}}$ definidas no Teorema 1.1. Então $u_{1} \leq u_{2}$ e $u_{1}$, respectivamente $u_{2}$ é a subsolução, respectivamente a supersolução de $\left(P_{\lambda}\right)$.

Mais ainda, como

$$
\left\{\begin{aligned}
-\Delta u_{1} & =\lambda_{1} u_{1}^{q}+u_{1}^{p}, & & x \in \Omega, \\
u_{1} & =0, & & x \in \partial \Omega
\end{aligned}\right.
$$

e

$$
\left\{\begin{aligned}
-\Delta u_{2} & =\lambda_{2} u_{2}^{q}+u_{2}^{p}, & & x \in \Omega, \\
u_{2} & =0, & & x \in \partial \Omega,
\end{aligned}\right.
$$

então temos

$$
\begin{array}{rlrl}
-\Delta\left(u_{2}-u_{1}\right) & =-\Delta u_{2}+\Delta u_{1}=\lambda_{2} u_{2}^{q}+u_{2}^{p}-\left(\lambda_{1} u_{1}^{q}+u_{1}^{p}\right) \\
& \geq \lambda_{1} u_{2}^{q}+u_{2}^{p}-\lambda_{1} u_{1}^{q}-u_{1}^{p} & & \\
& =\lambda_{1}\left(u_{2}^{q}-u_{1}^{q}\right)+\left(u_{2}^{p}-u_{1}^{p}\right) \geq 0, & & x \in \Omega, \\
u_{2}-u_{1} & =0, & & x \in \partial \Omega .
\end{array}
$$

Como $u_{1} \neq u_{2}$ (pois $\lambda_{1}<\lambda_{2}$ ), então pelo Lema de Hopf (ver Apêndice $A$ o Lema A.1), temos

$$
u_{1}<u_{2} \quad \text { e } \quad \frac{\partial}{\partial \nu}\left(u_{2}-u_{1}\right)<0,
$$

onde $\nu$ é o vetor normal unitário exterior a $\partial \Omega$. Agora, considere

$$
\begin{gathered}
\tilde{f}_{\lambda}(x, s)=\left\{\begin{array}{lr}
f_{\lambda}\left(u_{1}(x)\right), & s \leq u_{1}, \\
f_{\lambda}(s), & u_{1}<s<u_{2}, \\
f_{\lambda}\left(u_{2}(x)\right), & s \geq u_{2},
\end{array}\right. \\
\tilde{F}_{\lambda}(x, u)=\int_{0}^{u} \tilde{f}_{\lambda}(x, s) d s
\end{gathered}
$$

e o funcional $\tilde{I}_{\lambda}: H \rightarrow \mathbb{R}$ dado por,

$$
\tilde{I}_{\lambda}(u)=\frac{1}{2}\|u\|^{2}-\int_{\Omega} \tilde{F}_{\lambda}(x, u) d x .
$$


Como $u_{1}$ e $u_{2}$ são contínuas em $\bar{\Omega}$, então são limitadas em $\bar{\Omega}$. Portanto, $\tilde{f}_{\lambda}$ é uma função limitada, sendo assim, $\tilde{F}_{\lambda}(x, s) \leq C . s$, para todo $x \in \Omega$ e $t>0$. Pela imersão de $H$ em $L^{1}(\Omega)$ obtemos,

$$
\tilde{I}_{\lambda}(u) \geq \frac{1}{2}\|u\|^{2}-C\|u\|=\|u\|\left(\frac{1}{2}\|u\|-C\right),
$$

para toda $u \in H$. Logo, $\tilde{I}_{\lambda}$ é coercivo e limitado inferiormente. Assim, $\tilde{I}_{\lambda}$ possui um mínimo global $u_{\lambda} \in W^{2, p}(\Omega), \forall p<\infty$ e por sua vez $u_{\lambda}$ é ponto crítico para $\tilde{I}_{\lambda}$. Portanto,

$$
\left\{\begin{aligned}
-\Delta u_{\lambda} & =\tilde{f}_{\lambda}\left(x, u_{\lambda}\right), & & x \in \Omega, \\
u_{\lambda} & =0, & & x \in \partial \Omega .
\end{aligned}\right.
$$

Agora, pela definição de $\tilde{f}_{\lambda}$, temos

$$
f_{\lambda}\left(u_{2}\right) \geq \tilde{f}_{\lambda}\left(x, u_{\lambda}\right) \geq f_{\lambda}\left(u_{1}\right)
$$

Desse modo, como $u_{1}$ e $u_{2}$ são soluções de $\left(P_{\lambda}\right)$, temos

$$
\left\{\begin{array}{rlrl}
-\Delta\left(u_{1}-u_{\lambda}\right) & =-\Delta u_{1}+\Delta u_{\lambda}=\lambda_{1} u_{1}^{q}+u_{1}^{p}-\tilde{f}_{\lambda}\left(x, u_{\lambda}\right) & \\
& \leq \lambda u_{1}^{q}+u_{1}^{p}-\tilde{f}_{\lambda}\left(x, u_{\lambda}\right)=f_{\lambda}\left(x, u_{1}\right)-\tilde{f}_{\lambda}\left(x, u_{\lambda}\right) \leq 0, & & x \in \Omega, \\
u_{1}-u_{\lambda} & =0, & & x \in \partial \Omega
\end{array}\right.
$$

$$
\left\{\begin{array}{rlrl}
-\Delta\left(u_{\lambda}-u_{2}\right) & =-\Delta u_{\lambda}+\Delta u_{2}=\tilde{f}_{\lambda}\left(x, u_{\lambda}\right)-\left(\lambda_{2} u_{2}^{q}+u_{2}^{p}\right) & \\
& \leq \tilde{f}_{\lambda}\left(x, u_{\lambda}\right)-\left(\lambda u_{2}^{q}+u_{2}^{p}\right)=\tilde{f}_{\lambda}\left(x, u_{\lambda}\right)-f_{\lambda}\left(x, u_{2}\right) \leq 0, & & x \in \Omega, \\
u_{\lambda}-u_{2} & =0, & & x \in \partial \Omega .
\end{array}\right.
$$

Novamente, pelo Lema de Hopf, temos que

$$
\begin{cases}u_{1}<u_{\lambda}<u_{2}, & x \in \Omega, \\ \frac{\partial}{\partial \nu}\left(u_{\lambda}-u_{1}\right)<0, & x \in \partial \Omega, \\ \frac{\partial}{\partial \nu}\left(u_{\lambda}-u_{2}\right)<0, & x \in \partial \Omega .\end{cases}
$$

Pelo Lema A.2 (ver Apêndice $A$ ), $u_{\lambda} \in C^{2, \alpha}(\Omega), \alpha \in(0,1)$, assim, temos que $\left\|u-u_{\lambda}\right\|_{C^{1}} \leq \varepsilon$, para $\varepsilon>0$, suficientemente pequeno, temos que $u_{1}<u<u_{2}$, para todo $x \in \Omega$.

Dessa forma, por definição temos que

$$
\tilde{f}_{\lambda}(x, u)=f_{\lambda}(x, u),
$$

e consequentemente $\bar{I}_{\lambda}(u)=\tilde{I}_{\lambda}(u)$, para $u_{1}<u<u_{2}$. Portanto, $u_{\lambda}$ também é um minimizante local para $\bar{I}_{\lambda}$, na topologia $C^{1}$.

A seguir fixaremos o $\lambda$ e procuraremos uma segunda solução de $\left(P_{\lambda}\right)$ da forma $u=u_{0}+v$, onde $u_{0}$ denota a solução encontrada no Lema 1.7 e $v>0$. 
Dessa forma a correspondente equação para $v$ fica

$$
\begin{aligned}
-\Delta v & =-\Delta\left(u_{0}+v-u_{0}\right)=-\Delta\left(u_{0}+v\right)+\Delta u_{0} \\
& =\lambda\left(u_{0}+v\right)^{q}+\left(u_{0}+v\right)^{p}-\lambda u_{0}^{q}-u_{0}^{p} .
\end{aligned}
$$

Agora definimos

$$
\begin{gathered}
g(x, s)=g_{\lambda}(x, s)=\left\{\begin{array}{cc}
\lambda\left(u_{0}+s\right)^{q}-\lambda u_{0}^{q}+\left(u_{0}+s\right)^{p}-u_{0}^{p}, & s \geq 0, \\
0, & s<0,
\end{array}\right. \\
G(v)=G_{\lambda}(v)=\int_{0}^{v} g(x, s) d s \quad \text { e } \quad J(v)=J_{\lambda}(v)=\frac{1}{2}\|v\|^{2}-\int_{\Omega} G(v) d x .
\end{gathered}
$$

Se $v \in H, v \not \equiv 0$ é um ponto crítico de $J$, logo $v$ é solução de (1.39) e, pelo Princípio do Máximo, $v>0$ em $\Omega$. Onde $u=u_{0}+v$ é solução de $\left(P_{\lambda}\right)$ e $u \neq u_{0}$. Nós argumentamos por contradição e assumimos que $v=0$ é o único ponto crítico de $J$.

Lema 1.8. $v=0$ é um mínimo local de $J$ em $H$.

Demonstração. Como apresentado no Teorema A.12 (Apêndice A) é suficiente mostrar que $v=0$ é um mínimo local de $J$ na topologia de $C^{1}$. Assim, denotaremos por $v^{+}$a parte positiva de $v$.

Logo,

$$
\begin{aligned}
G\left(v^{+}\right)-F\left(u_{0}+v^{+}\right)= & \int_{0}^{v^{+}} g(x, s) d s-\int_{0}^{u_{0}+v^{+}} f_{\lambda}(s) d s \\
= & \int_{0}^{v+} \lambda\left(u_{0}+s\right)^{q}-\lambda u_{0}^{q}+\left(u_{0}+s\right)^{p}-u_{0}^{p} d s-\int_{0}^{u_{0}+v^{+}} \lambda s^{q}+s^{p} d s \\
= & {\left[\frac{\lambda}{q+1}\left(u_{0}+s\right)^{q+1}-\lambda u_{0}^{q} s+\frac{1}{p+1}\left(u_{0}+s\right)^{p+1}-u_{0}^{p} s\right]_{0}^{v^{+}} } \\
= & -\left[\frac{\lambda}{q+1} s^{q+1}+\frac{1}{p+1} s^{p+1}\right]_{0}^{u_{0}+v^{+}}\left(u_{0}+v^{+}\right)^{q+1}-\lambda u_{0}^{q} v^{+}+\frac{1}{p+1}\left(u_{0}+v^{+}\right)^{p+1}-u_{0}^{p} v^{+} \\
& \left.-\frac{\lambda}{q+1}\left(u_{0}+0\right)^{q+1}+\lambda u_{0}^{q} \cdot 0-\frac{1}{p+1}\left(u_{0}+0\right)^{p+1}+u_{0}^{p} \cdot 0\right] \\
= & -\left[\frac{\lambda}{q+1}\left(u_{0}+v^{+}\right)^{q+1}+\frac{1}{p+1}\left(u_{0}+v^{+}\right)^{p+1}-\frac{\lambda}{q+1} .0^{q+1}-\frac{1}{p+1} .0^{p+1}\right] \\
& \frac{\lambda+1}{q+1}\left(u_{0}+v^{+}\right)^{q+1}-\lambda u_{0}^{q} v^{+}+\frac{1}{p+1}\left(u_{0}+v^{+}\right)^{p+1}-u_{0}^{p} v^{+} \\
= & -\frac{\lambda}{q+1} u_{0}^{q+1}-\frac{1}{p+1} u_{0}^{p+1}-\frac{\lambda}{q+1}\left(u_{0}+v^{+}\right)^{q+1}-\frac{1}{p+1}\left(u_{0}+v^{+}\right)^{p+1} \\
& -\frac{1}{p+1} u_{0}^{p+1}-u_{0}^{p} v^{+} .
\end{aligned}
$$


Então,

$$
\begin{aligned}
J(v)= & \frac{1}{2}\left\|v^{+}\right\|^{2}+\frac{1}{2}\left\|v^{-}\right\|^{2}-\int_{\Omega} G\left(v^{+}\right) d x \\
= & \frac{1}{2}\left\|v^{+}\right\|^{2}+\frac{1}{2}\left\|v^{-}\right\|^{2}-\int_{\Omega} F\left(u_{0}+v^{+}\right) d x \\
& +\int_{\Omega}\left[\frac{\lambda}{q+1} u_{0}^{q+1}+\lambda u_{0}^{q} v^{+}+\frac{1}{p+1} u_{0}^{p+1}+u_{0}^{p} v^{+}\right] d x \\
= & \frac{1}{2}\left\|v^{+}\right\|^{2}+\frac{1}{2}\left\|v^{-}\right\|^{2}-\int_{\Omega} F\left(u_{0}+v^{+}\right) d x \\
& +\int_{\Omega} F\left(u_{0}\right) d x+\int_{\Omega}\left(\lambda u_{0}^{q}+u_{0}^{p}\right) v^{+} d x .
\end{aligned}
$$

Por outro lado, temos

$$
\begin{aligned}
\bar{I}_{\lambda}\left(u_{0}+v^{+}\right) & =\frac{1}{2}\left\|u_{0}+v^{+}\right\|^{2}-\int_{\Omega} F_{\lambda}\left(u_{0}+v^{+}\right) d x \\
& =\frac{1}{2} \int_{\Omega}\left|\nabla\left(u_{0}+v^{+}\right)\right|^{2} d x-\int_{\Omega} F_{\lambda}\left(u_{0}+v^{+}\right) d x \\
& =\frac{1}{2}\left[\int_{\Omega}\left|\nabla u_{0}\right|^{2}+\left|\nabla v^{+}\right|^{2}+2 \nabla u_{0} \nabla v^{+} d x\right]-\int_{\Omega} F_{\lambda}\left(u_{0}+v^{+}\right) d x \\
& =\frac{1}{2} \int_{\Omega}\left|\nabla u_{0}\right|^{2} d x+\frac{1}{2} \int_{\Omega}\left|\nabla v^{+}\right|^{2} d x+\int_{\Omega} \nabla u_{0} \nabla v^{+} d x-\int_{\Omega} F_{\lambda}\left(u_{0}+v^{+}\right) d x \\
& =\frac{1}{2}\left\|u_{0}\right\|^{2}+\frac{1}{2}\left\|v^{+}\right\|^{2}+\left[\int_{\Omega} \frac{\partial u_{0}}{\partial \eta} \cdot v^{+} d S-\int_{\Omega} \Delta u_{0} v^{+} d x\right]-\int_{\Omega} F_{\lambda}\left(u_{0}+v^{+}\right) d x \\
& =\frac{1}{2}\left\|u_{0}\right\|^{2}+\frac{1}{2}\left\|v^{+}\right\|^{2}+\int_{\Omega}\left(-\Delta u_{0}\right) v^{+} d x-\int_{\Omega} F_{\lambda}\left(u_{0}+v^{+}\right) d x \\
& =\frac{1}{2}\left\|u_{0}\right\|^{2}+\frac{1}{2}\left\|v^{+}\right\|^{2}+\int_{\Omega}\left(\lambda u_{0}^{q}+u_{0}^{p}\right) v^{+} d x-\int_{\Omega} F_{\lambda}\left(u_{0}+v^{+}\right) d x
\end{aligned}
$$

Assim,

$$
J(v)=\frac{1}{2}\left\|v^{+}\right\|^{2}+\frac{1}{2}\left\|v^{-}\right\|^{2}-\int_{\Omega} F\left(u_{0}+v^{+}\right) d x+\int_{\Omega} F\left(u_{0}\right) d x+\int_{\Omega}\left(\lambda u_{0}^{q}+u_{0}^{p}\right) v^{+} d x .
$$

Substituindo a expressão $-\int_{\Omega} F_{\lambda}\left(u_{0}+v^{+}\right) d x$ de (1.42) em (1.43) temos

$$
\begin{aligned}
J(v) & =\frac{1}{2}\left\|v^{+}\right\|^{2}+\frac{1}{2}\left\|v^{-}\right\|^{2}+\left[\bar{I}_{\lambda}\left(u_{0}+v^{+}\right)-\frac{1}{2}\left\|u_{0}\right\|^{2}-\frac{1}{2}\left\|v^{+}\right\|^{2}-\int_{\Omega}\left(\lambda u_{0}^{q}+u_{0}^{p}\right) v^{+} d x\right] \\
& +\int_{\Omega} F\left(u_{0}\right) d x+\int_{\Omega}\left(\lambda u_{0}^{q}+u_{0}^{p}\right) d x \\
& =\frac{1}{2}\left\|v^{-}\right\|^{2}+\bar{I}_{\lambda}\left(u_{0}+v^{+}\right)-\frac{1}{2}\left\|u_{0}\right\|^{2}+\int_{\Omega} F\left(u_{0}\right) d x \\
& =\frac{1}{2}\left\|v^{-}\right\|^{2}+\bar{I}_{\lambda}\left(u_{0}+v^{+}\right)-\left[\frac{1}{2}\left\|u_{0}\right\|^{2}-\int_{\Omega} F\left(u_{0}\right) d x\right] \\
& =\frac{1}{2}\left\|v^{-}\right\|^{2}+\bar{I}_{\lambda}\left(u_{0}+v^{+}\right)-\bar{I}_{\lambda}\left(u_{0}\right) .
\end{aligned}
$$

Logo, $J(v) \geq 0$, pois pelo Lema $1.7 u_{0}$ é um minimizante local para $\bar{I}_{\lambda}$. Daí existe $\varepsilon>0$ tal que $\bar{I}_{\lambda}\left(u_{0}+v^{+}\right)-\bar{I}_{\lambda}\left(u_{0}\right) \geq 0$, para $\left\|v^{+}\right\|_{C^{1}} \leq \varepsilon$.

Assim, $v$ é um mínimo local na topologia $C^{1}$. Logo pelo Teorema A.12, segue que $v$ é um mínimo 
local em $H$.

Lembre-se que $J$ satisfaz a condição de Palais- Smale no nível $c \in \mathbb{R}$, denotada por $(P S)_{c}$, se para toda sequência $\left(u_{n}\right) \subset H$ satisfazendo

$$
\left|J\left(u_{n}\right)\right| \leq c \quad \text { e } \quad J^{\prime}\left(u_{n}\right) \rightarrow 0
$$

possui subsequência convergente.

Lema 1.9. 1. Se $p<\frac{N+2}{N-2} \operatorname{logo} J_{\lambda}$ satisfaz $(P S)_{c}$ para todo $c \in \mathbb{R}$.

2. Se $p=\frac{N+2}{N-2}$ e se 0 é o único ponto crítico de $J_{\lambda}$, então $J_{\lambda}$ satisfaz $(P S)_{c}$ para todo $c<\frac{1}{N} S^{\frac{N}{2}}$, onde $S$ denota a melhor constante de Sobolev.

Demonstração. Verificação para o caso $p<\frac{N+2}{N-2}$.

Seja $\left(u_{n}\right) \subset H$ uma sequência $(P S)_{c}$ para $J_{\lambda}$, isto é

$$
J_{\lambda}\left(u_{n}\right) \rightarrow c \quad \text { e } \quad J_{\lambda}^{\prime}\left(u_{n}\right) \rightarrow 0 \quad \text { em } H^{-1}
$$

assim

$$
J_{\lambda}\left(u_{n}\right)=\frac{1}{2}\left\|u_{n}^{-}\right\|^{2}+I_{\lambda}\left(u_{0}+u_{n}^{+}\right)-I_{\lambda}\left(u_{0}\right) \rightarrow c
$$

e

$$
J_{\lambda}^{\prime}\left(u_{n}\right) u_{n}^{-}=\left\|u_{n}^{-}\right\|^{2}+I_{\lambda}^{\prime}\left(u_{0}+u_{n}^{+}\right) u_{n}^{-}-I_{\lambda}^{\prime}\left(u_{0}\right) u_{n}^{-} \rightarrow 0 .
$$

Mas como $u_{0}$ é ponto crítico do funcional $I_{\lambda}$, temos em (1.47) que

$$
J_{\lambda}^{\prime}\left(u_{n}\right) u_{n}^{-}=\left\|u_{n}^{-}\right\|^{2}+I_{\lambda}^{\prime}\left(u_{0}+u_{n}^{+}\right) u_{n}^{-} \rightarrow 0
$$

$\log$,

$$
\left\|u_{n}^{-}\right\|^{2} \rightarrow 0 \quad \text { e } \quad I_{\lambda}^{\prime}\left(u_{0}+u_{n}^{+}\right) u_{n}^{-} \rightarrow 0 .
$$

Por outro lado, de (1.46) e usando o fato que $\left\|u_{n}^{-}\right\|^{2} \rightarrow 0$, obtida em (1.48), temos

$$
I_{\lambda}\left(u_{0}+u_{n}^{+}\right)-I_{\lambda}\left(u_{0}\right) \rightarrow c
$$

assim

$$
I_{\lambda}\left(u_{0}+u_{n}^{+}\right) \rightarrow c+I_{\lambda}\left(u_{0}\right)
$$

De (1.48) e (1.49) temos que $\left(u_{0}+u_{n}\right)$ é uma sequência $(P S)$ para $I_{\lambda}$, no nível $c+I_{\lambda}\left(u_{0}\right)$. Mas como $I_{\lambda}$ satisfaz $(P S)$ (ver Apêndice $A$ o Lema A.3). Logo, segue que $\left(w_{n}\right):=\left(u_{0}+u_{n}\right)$ possui subsequência convergente, isto é, existe $\left(w_{n_{j}}\right) \subset\left(w_{n}\right)$ convergente. Assim, $u_{n_{j}}=w_{n_{j}}-u_{0}$ é uma subsequência convergente. Portanto, $J_{\lambda}$ satisfaz a condição de $(P S)_{c}$.

Daremos agora uma ideia da mesma prova apresentada acima, contudo, usaremos a condição de Ambrosetti-Rabinowitz (A-R).

A prova baseia-se no artigo dado em [3], então invocando o artigo mencionado, temos as seguintes condições: 
$\left(g_{1}\right)|g(x, s)| \leq C_{1}+C_{2}|s|^{p}$, onde $1<p<\frac{N+2}{N-2}$,

$\left(g_{2}\right)$ Se $G(u)=\int_{0}^{u} g(x, s) d s$, logo existe $R>0, \mu>2$ tal que $G(u) \leq \frac{1}{\mu} g(x, u) u, \forall|u| \geq R, x \in \bar{\Omega}$.

Finalmente temos o seguinte resultado como em [3]:

Resultado: Se g satisfaz $\left(g_{1}\right)$ e $\left(g_{2}\right)$, logo o funcional $J_{\lambda}$ satisfaz a condiçãa $(P S)$.

Assim, se verificamos as condições $\left(g_{1}\right)$ e $\left(g_{2}\right)$ para nossa função $g$ teríamos pelo resultado dado em [3], que $J_{\lambda}$ satisfaz $(P S)$.

\section{Verificando as condições $\left(g_{1}\right)$ e $\left(g_{2}\right)$.}

1. Condição $\left(g_{1}\right)$ :

Sendo

$$
g(x, s)=\left\{\begin{array}{cc}
\lambda\left(u_{0}+s\right)^{q}-\lambda u_{0}^{q}+\left(u_{0}+s\right)^{p}-u_{0}^{p}, & s \geq 0 \\
0, & s<0 .
\end{array}\right.
$$

Temos que

$$
\begin{aligned}
|g(x, s)| & =\left|\lambda\left(u_{0}+s\right)^{q}-\lambda u_{0}^{q}+\left(u_{0}+s\right)^{p}-u_{0}^{p}\right| \\
& \leq \lambda\left|u_{0}+s\right|^{q}+\lambda\left|u_{0}\right|^{q}+\left|u_{0}+s\right|^{p}+\left|u_{0}\right|^{p} \\
& \leq \lambda\left|u_{0}+s\right|^{p}+\lambda\left|u_{0}\right|^{p}+\left|u_{0}+s\right|^{p}+\left|u_{0}\right|^{p} \\
& =(\lambda+1)\left|u_{0}+s\right|^{p}+(\lambda+1)\left|u_{0}\right|^{p} \\
& =a_{1}+(\lambda+1)\left|u_{0}+s\right|^{p} \\
& \leq a_{1}+(\lambda+1) \cdot C\left(u_{0}^{p}+s^{p}\right) \\
& \leq C_{1}+C_{2}|s|^{p} .
\end{aligned}
$$

Portanto, existem as constantes $C_{1}$ e $C_{2}$ tal que a desigualdade em $\left(g_{1}\right)$ é satisfeita.

2. Condição $\left(g_{2}\right)$ :

Note que,

$$
\lim _{u \rightarrow \infty} \frac{G(x, u)}{g(x, u) u}=\lim _{u \rightarrow+\infty}\left(\frac{\frac{\lambda\left(u_{0}+u\right)^{q+1}}{q+1}-\lambda u_{0}^{q} u+\frac{\left(u_{0}+u\right)^{p+1}}{p+1}-u_{0}^{p} u-\frac{\lambda u_{0}^{q+1}}{q+1}-\frac{u_{0}^{p+1}}{p+1}}{\lambda\left(u_{0}+u\right)^{q} u-\lambda u_{0}^{q} u+\left(u_{0}+u\right)^{p} u-u_{0}^{p} u}\right),
$$

como temos uma indeterminação, aplicamos L'Hospital. Logo temos,

$$
\lim _{u \rightarrow+\infty}\left(\frac{\lambda\left(u_{0}+u\right)^{q}-\lambda u_{0}^{q}+\left(u_{0}+u\right)^{p}-u_{0}^{p}}{\lambda q\left(u_{0}+u\right)^{q-1} u+\lambda\left(u_{0}+u\right)^{q}-\lambda u_{0}^{q}+p\left(u_{0}+u\right)^{p-1} u+\left(u_{0}+u\right)^{p}-u_{0}^{p}}\right) .
$$

Novamente temos uma indeterminação, assim aplicando L'Hospital mais uma vez, temos

$$
\lim _{u \rightarrow+\infty}\left(\begin{array}{c}
\frac{\lambda\left(u_{0}+u\right)^{q-1}+p\left(u_{0}+u\right)^{p-1}}{\lambda q(q-1)\left(u_{0}+u\right)^{q-2} u+\lambda q\left(u_{0}+u\right)^{q-1}} \\
+\lambda q\left(u_{0}+u\right)^{q-1}+p(p-1)\left(u_{0}+u\right)^{p-2} u \\
+2 p\left(u_{0}+u\right)^{p-1}
\end{array}\right) .
$$


Agora, note que como $0<q<1$, os termos que tem dependência de $q$ tendem a zero. Por outro lado, note que o termo $p\left(u_{0}+u\right)^{p-1}$, no numerador aparece uma vez, e no denominador, duas vezes acompanhado do termo $p(p-1)\left(u_{0}+u\right)^{p-2} u$, assim o termo do denominador cresce mais rápido que o numerador, fazendo tender a zero toda a expressão. Para que fique mais claro, aplicaremos L'Hospital novamente nos termos com dependência de $p$, pois para os termos que contém $q$, esses tendem a zero. Assim temos,

$$
\lim _{u \rightarrow+\infty}\left(\frac{p(p-1)\left(u_{0}+u\right)^{p-2}}{p(p-1)(p-2)\left(u_{0}+u\right)^{p-3} u+3 p(p-1)\left(u_{0}+u\right)^{p-2}}\right)=\frac{1}{p+1},
$$

é claro assim que, o termo do denominador cresce mais rápido que o numerador, concluindo que $G(u) \leq \frac{1}{\mu} g(u) u$, com $\mu=p+1$. Portanto, como as condições do resultado são satisfeitas, $J_{\lambda}$ satisfaz $(P S)$. De fato, seja $\left(u_{n}\right) \subset H$ uma sequência tal que

$$
J_{\lambda}\left(u_{n}\right) \rightarrow c \quad \text { e } \quad J_{\lambda}^{\prime}\left(u_{n}\right) \rightarrow 0
$$

Agora, como

$$
J_{\lambda}\left(u_{n}\right)=\frac{1}{2}\left\|u_{n}\right\|^{2}-\int_{\Omega} G_{\lambda}\left(u_{n}\right) d x
$$

e usando a condição $\left(g_{2}\right)$, temos que

$$
c \geq \frac{1}{2}\left\|u_{n}\right\|^{2}-\int_{\Omega} G_{\lambda}\left(u_{n}\right) d x \geq \frac{1}{2}\left\|u_{n}\right\|-\frac{1}{\mu} \int_{\Omega} g_{\lambda}\left(u_{n}\right) u_{n} d x .
$$

Desde que, como $J_{\lambda}^{\prime}\left(u_{n}\right) \rightarrow 0$, temos que para todo $\varepsilon>0$, existe $N=N(\varepsilon)>0$ tal que para todo $n \geq N$,

$$
\left|J_{\lambda}^{\prime}\left(u_{n}\right) u_{n}\right| \leq \varepsilon\left\|u_{n}\right\|
$$

Tomando $\varepsilon=1$ e combinando-se (1.50) e (1.51), temos que

$$
\begin{aligned}
c+o_{n}(1) & =J_{\lambda}\left(u_{n}\right)-\frac{1}{\mu} J_{\lambda}^{\prime}\left(u_{n}\right) u_{n} \\
& \geq \frac{1}{2}\left\|u_{n}\right\|^{2}-\frac{1}{\mu} \int_{\Omega} g_{\lambda}\left(u_{n}\right) u_{n} d x-\frac{1}{\mu}\left\|u_{n}\right\| \\
& \geq\left(\frac{1}{2}-\frac{1}{\mu}\right)\left\|u_{n}\right\|^{2}-C-\frac{1}{\mu}\left\|u_{n}\right\| .
\end{aligned}
$$

Então $\left(u_{n}\right)$ é limitada em $H$, assim possui subsequência convergente.

Verificação para o caso $p=\frac{N+2}{N-2}$.

Seja $\left(w_{n}\right) \subset H$ uma sequência tal que

$$
J_{\lambda}^{\prime}\left(w_{n}\right) \rightarrow 0 \quad \text { e } \quad J_{\lambda}\left(w_{n}\right) \rightarrow c<\frac{S^{N / 2}}{N}
$$

Note que $w_{n}$ é limitada pois,

$$
\frac{1}{p+1} J_{\lambda}^{\prime}\left(w_{n}\right)\left(u_{0}+w_{n}\right)-J_{\lambda}\left(w_{n}\right) \leq \varepsilon_{n}\left\|u_{0}+w_{n}\right\| ; \quad \varepsilon_{n} \rightarrow 0 .
$$


Na expressão acima, os termos de potência $p+1$ são cancelados, logo pode-se escrever como

$$
\left\|w_{n}\right\|^{2} \leq C\left(\left\|w_{n}\right\|^{q+1}+\left\|w_{n}\right\|+1\right)
$$

assim, $\left\|w_{n}\right\|$ é limitada. Passando a uma subsequência se necessário, $w_{n} \rightarrow w_{\lambda}$ em $H, w_{n} \rightarrow w_{\lambda}$ em $L^{r}, 1<r<2^{*}$.

Mais ainda, $u_{0}+w_{\lambda}$ é solução de $\left(P_{\lambda}\right)$ e assim ponto crítico de $J_{\lambda}$. Então

$$
\frac{1}{p+1} J_{\lambda}^{\prime}\left(w_{n}\right)\left(u_{0}+w_{n}\right)-J_{\lambda}\left(w_{n}\right)=\frac{1}{N}\left\|w_{n}\right\|^{2}+o_{n}(1) \rightarrow c .
$$

Se $c=0, \operatorname{logo} w_{n} \rightarrow 0$ em $H$ e a prova está concluída.

Afirmação 1.2. $c=0$ é a única possibilidade.

De fato, suponha por contradição que $c \neq 0$. Podemos então supor que $\left\|w_{n}\right\|$ converge e como $I_{\lambda}^{\prime}\left(w_{n}\right) w_{n} \rightarrow 0$, concluímos que

$$
\lim _{n \rightarrow \infty}\left\|w_{n}\right\|^{2}=\lim _{n \rightarrow \infty} \int_{\Omega}\left(w_{n}^{+}\right)^{2^{*}} d x=N c .
$$

Por definição de $S$ (ver Apêndice $A$ expressão em A.21), temos que

$$
\left\|w_{n}\right\|^{2} \geq S\left(\int_{\Omega}\left|w_{n}\right|^{2^{*}} d x\right)^{2 / 2^{*}}
$$

passando ao limite obtemos

$$
c N \geq S(c N)^{2 / 2^{*}}
$$

e $\operatorname{assim} c \geq \frac{S^{N / 2}}{N}$, se $c>0$. Uma contradição com o Lema A.6 (ver Apêndice $A$ ).

Portanto, $J_{\lambda}$ satisfaz $(P S)_{c}$ para todo $c<\frac{S^{N / 2}}{N}$.

Podemos agora apresentar a demonstração do Teorema 1.3.

\section{Demonstração do Teorema 1.3}

1. CASO $p<\frac{N+2}{N-2}$

Primeiramente estudaremos o caso em que $1<p<\frac{N+2}{N-2}$, onde temos que ocorre $(P S)_{c}$ para todo c. Mostraremos que do fato que $1<p$, segue que para todo $v>0$ teremos que $J_{\lambda}(t v) \rightarrow-\infty$ quando $t \rightarrow+\infty$. Assim, seja $v \in H$. Desejamos aplicar o Teorema do Passo da Montanha (ver Apêndice $B$ o Teorema $B .1$ ), para isso, primeiramente verificaremos que existe $v \notin B_{r_{0}}$ tal que $J_{\lambda}(v) \leq 0$.

Temos de modo direto que $J(0)=0<\varepsilon$, pois pela expressão em (1.38) temos $J_{\lambda}(0)=\frac{1}{2}\|0\|^{2}$ $+\bar{I}_{\lambda}\left(u_{0}+0\right)-\bar{I}_{\lambda}\left(u_{0}\right)=0$. 
Agora, fixe $v \in H, v^{+} \neq 0$ e escreva $w:=t v$ para $t>0$, assim temos que

$$
J_{\lambda}(w)=\frac{1}{2}\|w\|^{2}-\int_{\Omega} G(w) d x
$$

$\log O$

$$
\begin{aligned}
& J_{\lambda}(t v)=\frac{1}{2}\|t v\|^{2}-\int_{\Omega} G(t v) d x \\
& =\frac{t^{2}}{2}\|v\|^{2}-\int_{\Omega} \int_{0}^{t v^{+}} g(x, s) d s d x \\
& =\frac{t^{2}}{2}\|v\|^{2}-\int_{\Omega} \int_{0}^{t v^{+}} \lambda\left(u_{0}+s\right)^{q}-\lambda u_{0}^{q}+\left(u_{0}+s\right)^{p}-u_{0}^{p} d s d x \\
& \left.=\frac{t^{2}}{2}\|v\|^{2}-\int_{\Omega} \int_{0}^{t v^{+}} \lambda\left(u_{0}+s\right)^{q}+\left(u_{0}+s\right)^{p}-\left(\lambda u_{0}^{q}+u_{0}\right)^{p}\right) d s d x \\
& =\frac{t^{2}}{2}\|v\|^{2}-\int_{\Omega}\left(\frac{\lambda}{q+1}\left(u_{0}+t v^{+}\right)^{q+1}-\frac{\lambda}{q+1} u_{0}^{q+1}+\frac{1}{p+1}\left(u_{0}+t v^{+}\right)^{p+1}\right. \\
& \left.-\frac{1}{p+1} u_{0}^{p+1}+t v^{+} f_{\lambda}\left(u_{0}\right)\right) d x \\
& =\frac{t^{2}}{2}\|v\|^{2}-t^{q+1} \int_{\Omega} \frac{\lambda}{q+1}\left(\frac{u_{0}+t v^{+}}{t}\right)^{q+1} d x-t^{p+1} \int_{\Omega} \frac{1}{p+1}\left(\frac{u_{0}+t v^{+}}{t}\right)^{p+1} d x \\
& +\int_{\Omega}\left(\frac{\lambda}{q+1} u_{0}^{q+1}+\frac{1}{p+1} u_{0}^{p+1}\right) d x+t \int_{\Omega} f_{\lambda}\left(u_{0}\right) v^{+} d x \\
& =\frac{t^{2}}{2}\|v\|^{2}-t^{q+1} \int_{\Omega} \frac{\lambda}{q+1}\left(\frac{u_{0}+t v^{+}}{t}\right)^{q+1} d x-t^{p+1} \int_{\Omega} \frac{1}{p+1}\left(\frac{u_{0}+t v^{+}}{t}\right)^{p+1} d x \\
& +\int_{\Omega} F_{\lambda}\left(u_{0}\right) d x+t \int_{\Omega} f_{\lambda}\left(u_{0}\right) v^{+} d x \\
& \leq \frac{t^{2}}{2}\|v\|^{2}-t^{q+1} \int_{\Omega} \frac{\lambda}{q+1}\left(\frac{u_{0}}{t}+v^{+}\right)^{q+1} d x-t^{p+1} \int_{\Omega} \frac{1}{p+1}\left(\frac{u_{0}}{t}+v^{+}\right)^{p+1} d x \\
& +C_{1}|\Omega|+t C_{2}|\Omega|
\end{aligned}
$$

onde $F_{\lambda}\left(u_{0}\right) \leq C_{2}$ e $f_{\lambda}\left(u_{0}\right) \leq C_{1}$, em que $C_{1}, C_{2}$ são constantes reais positivas. Agora como $q+1<2<p+1$, segue que

$$
J_{\lambda}(w)=J_{\lambda}(t v) \rightarrow-\infty \quad \text { quando } \quad t \rightarrow+\infty .
$$

Assim, existe $t_{0}>0$ tal que

$$
\|w\|=\left\|t_{0} v\right\|>r_{0} \quad \text { e } \quad J_{\lambda}(w)=J_{\lambda}\left(t_{0} v\right) \leq 0 .
$$

Por outro lado, note que

$$
\begin{aligned}
J_{\lambda}(t v)= & \frac{t^{2}}{2}\|v\|^{2}-t^{q+1} \int_{\Omega} \frac{\lambda}{q+1}\left(\frac{u_{0}+t v^{+}}{t}\right)^{q+1} d x-t^{p+1} \int_{\Omega} \frac{1}{p+1}\left(\frac{u_{0}+t v^{+}}{t}\right)^{p+1} d x \\
& +\int_{\Omega} F_{\lambda}\left(u_{0}\right) d x+t \int_{\Omega} f_{\lambda}\left(u_{0}\right) v^{+} d x
\end{aligned}
$$


e como todos os integrandos são positivos, temos que quando fizermos $t \rightarrow 0^{+}$, a potência que domina é 1 no caso "t", assim existe uma função $v_{\lambda} \in H, v_{\lambda} \neq 0$, tal que $J_{\lambda}\left(v_{\lambda}\right)=c \geq \varepsilon>0$ e $J_{\lambda}^{\prime}\left(v_{\lambda}\right) v_{\lambda}=0$. Isto é, $v_{\lambda}$ é um ponto crítico não trivial para $J_{\lambda}$.

Uma vez que $J_{\lambda}\left(u_{\lambda}\right)<0$, onde $u_{\lambda}$ é a primeira solução obtida pelo Teorema (1.1) e $J_{\lambda}\left(v_{\lambda}\right)>0$ temos que $u_{\lambda} \neq v_{\lambda}$ e $v_{\lambda}$ é a segunda solução para o problema $\left(P_{\lambda}\right)$, isso decorre o Teorema do Passo da Montanha, além do fato que

$$
c:=\inf _{\gamma \in \Gamma} \max \{J(\gamma(t)): t \in[0,1]\}
$$

onde

$$
\Gamma=\{\gamma \in C([0,1], H): \gamma(0)=0, \gamma(1) \leq 0\}
$$

2. CASO $p=\frac{N+2}{N-2}$

Vamos mostrar que o funcional $J_{\lambda}$ tem um ponto crítico não trivial no caso $p=2^{*}-1$. Novamente, $J_{\lambda}$ possui um mínimo local na origem e pode-se encontrar $e \in H$, com $\|e\|$ suficientemente grande, tal que $J_{\lambda}(e) \leq 0$.

Suponha por contradição que 0 é o único ponto crítico de $J_{\lambda}$. Considere o Teorema do Passo da Montanha no nível

$$
c_{\lambda}:=\inf _{\gamma \in \Gamma} \max _{t \in[0,1]} J_{\lambda}(\gamma(t))
$$

onde

$$
\Gamma=\left\{\gamma \in C\left([0,1], H_{0}^{1}\right) \mid \gamma(0)=0, \gamma(1)=e\right\} .
$$

Para aplicar o Teorema do Passo da Montanha, considere $w_{n} \in H$ tal que

$$
J_{\lambda}^{\prime}\left(w_{n}\right) \rightarrow 0 \quad \text { e } \quad J_{\lambda}\left(w_{n}\right) \rightarrow c_{\lambda}
$$

onde $0 \leq c_{\lambda}<\frac{S^{N / 2}}{N}$, pelo Lema A.6 (ver Apêndice A).

O Lema (1.9) implica que $w_{n} \rightarrow w_{\lambda}$ em $H$. Então $w_{\lambda}$ é um ponto crítico de $J_{\lambda}$, e assim, $w_{\lambda}=0$ por suposição. Logo temos por (1.53) que

$$
\frac{1}{p+1} J_{\lambda}^{\prime}\left(w_{n}\right)\left(u_{0}+w_{n}\right)-J_{\lambda}\left(w_{n}\right)=\frac{1}{N}\left\|w_{n}\right\|^{2}+o_{n}(1) \rightarrow c_{\lambda}
$$

Note que se $c_{\lambda}=0$, pode-se tomar $w_{n}$ satisfazendo $\left\|w_{n}\right\| \rightarrow r, r>0$ o que contradiz (1.54). Assim, $c_{\lambda}>0$. Como $J_{\lambda}^{\prime}\left(w_{n}\right)\left(w_{n}\right) \rightarrow 0$, concluímos que

$$
\lim _{n \rightarrow \infty}\left\|w_{n}\right\|^{2}=\lim _{n \rightarrow \infty} \int_{\Omega}\left(w_{n}^{+}\right)^{2^{*}} d x=N c_{\lambda} .
$$

Por definição de $S$ temos

$$
\left\|w_{n}\right\|^{2} \geq S\left(\left|w_{n}\right|^{2^{*}}\right)^{2 / 2^{*}}
$$

Passando ao limite, temos

$$
c_{\lambda} N \geq S\left(c_{\lambda} N\right)^{2 / 2^{*}}
$$

Segue que $c_{\lambda} \geq \frac{S^{N / 2}}{N}$, se $c_{\lambda}>0$. O que contradiz o Lema A.6 (ver Apêndice $A$ ). 
Logo $J_{\lambda}$ possui ponto crítico não trivial.

Observação 1.4. Nos teoremas anteriores as soluções encontradas estão em H. Mas sabe-se que por [6], as soluções pertencem a todo $L^{p}(\Omega)$ e então as soluções são clássicas.

Observação 1.5. No caso $q=0$ o problema $\left(P_{\lambda}\right)$ é

$$
\left\{\begin{aligned}
-\Delta u & =\lambda+u^{p}, & & x \in \Omega, \\
u & =0, & & x \in \partial \Omega,
\end{aligned}\right.
$$

e a existência de ao menos duas soluções positivas quando $p=\frac{N+2}{N-2}$ forem estabelecidas em [23] para $\lambda>0$ suficientemente pequeno.

Os resultados acima, sugerem que a estrutura do conjunto de soluções positivas de $\left(P_{\lambda}\right)$ olha-se como (ver Figura 1.1).
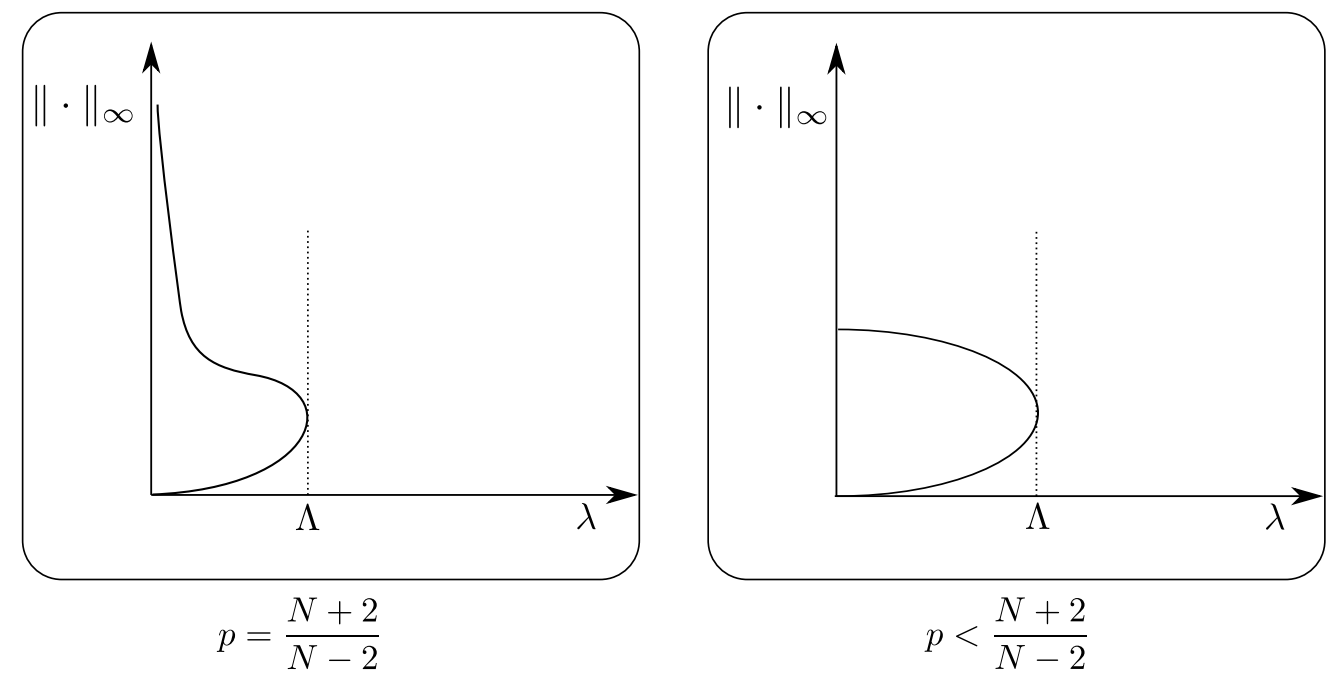

Figura 1.1: Representação.

\subsection{Existência de infinitas soluções}

Nesta seção consideraremos o caso particular de $\left(P_{\lambda}\right)$

$$
(P) \quad\left\{\begin{aligned}
-\Delta u & =\lambda|u|^{q-1} u+|u|^{p-1} u, & & x \in \Omega, \\
u & =0, & & x \in \partial \Omega,
\end{aligned}\right.
$$

e mostraremos que para $\lambda>0$ e pequeno, o problema $(P)$ tem infinitas soluções. Mais precisamente, a presença do termo sublinear $|u|^{q-1} u$ produz a existência de infinitas soluções de $(P)$, com energia negativa, sempre que $\lambda>0$ é perto do zero. Por outro lado, o termo $|u|^{p-1} u$ tem um papel importante produzindo a existência de infinitas soluções com energia positiva, sempre que $p<\frac{N+2}{N-2}$.

Apresentaremos agora um importante resultado para essa seção. 
Teorema 1.4. 1. Seja $0<q<1<p \leq \frac{N+2}{N-2}$. Então existe $\lambda^{*}>0$ tal que para todo $\lambda \in\left(0, \lambda^{*}\right) o$ problema $(P)$ tem infinitas soluções tal que $I_{\lambda}(u)<0$.

2. Se $0<q<1<p<\frac{N+2}{N-2}$. Então para todo $\lambda \in\left(0, \lambda^{*}\right)$ o problema $(P)$ tem também infinitas soluções tal que $I_{\lambda}(u)>0$.

Temos que se $p \leq \frac{N+2}{N-2}$ e para $u \in H$, definimos

$$
I(u)=I_{\lambda}(u)=\frac{1}{2}\|u\|^{2}-\frac{\lambda}{q+1}\|u\|_{q+1}^{q+1}-\frac{1}{p+1}\|u\|_{p+1}^{p+1} .
$$

Os pontos críticos de $I_{\lambda}$ sobre $H$ são soluções de $(P)$.

Seja $B_{r}=\{u \in H:\|u\| \leq r\}$. Usando a desigualdades de Sobolev e Hölder temos

$$
I_{\lambda}(u) \geq \frac{1}{2}\|u\|^{2}-\lambda C_{1}\|u\|^{q+1}-C_{2}\|u\|^{p+1} .
$$

De (1.55) prontamente encontramos que existe $\lambda^{*}>0$ tal que para todo $\lambda \in\left(0, \lambda^{*}\right]$ existem $r, a>0$ tal que

(I.1) $I_{\lambda}(u) \geq a$ para todo $\|u\|=r$

(I.2) $I_{\lambda}$ é limitada sobre $B_{r}$;

(I.3) $I_{\lambda}$ satisfaz $(P S)$ sobre $B_{r}$.

Agora fixamos $\lambda \in\left(0, \lambda^{*}\right]$, e daremos a prova do Teorema 1.4 item 1 .

Demonstração. 1. Seja

$$
\Sigma=\{A \subset H: 0 \notin A, u \in A \Rightarrow-u \in A\}
$$

Para $A \in \Sigma$ o gênero $Z_{2}$ de $A$ é denotado por $\gamma(A)$ (ver Apêndice $A .5$ ). Temos também

$$
\mathcal{A}_{n, r}=\left\{A \in \Sigma: A \text { é compacto }, A \subset B_{r}, \gamma(A) \geq n\right\}
$$

Claramente, $\mathcal{A}_{n, r} \neq \emptyset$ para todo $n=1,2, \cdots$, pois

$$
S_{n, \varepsilon}:=\partial\left(H_{n} \cap B_{\varepsilon}\right) \in \mathcal{A}_{n, r}
$$

Onde $H_{n}$ denota o subespaço $n$ - dimensional de $H$. Seja

$$
b_{n, r}=\inf _{A \in \mathcal{A}_{n, r}} \max _{u \in A} I(u)
$$

Cada $b_{n, r}$ é finito devido a (I.2). Mais ainda, temos que

$$
b_{n, r}<0, \quad \forall n \in N \text {. }
$$


De fato, seja $w \in H_{n}$ tal que $\|w\|=\varepsilon$. Uma vez que

$$
I(w) \leq \frac{1}{2} \varepsilon^{2}-\lambda C_{1} \varepsilon^{q+1},
$$

segue que $I(w)<0$ para $\varepsilon>0$ suficientemente pequeno. Assim $b_{n, r}<0$.

A seguir, note que $u \in B_{r} \cap\{I \leq 0\}$ o fluxo de maior descida $\eta_{t}$ (definida através do campo vetorial pseudo-gradiente (ver Lema de Deformação em B.2 do Apêndice B) está bem definida para $t \in[0, \infty)$ e

$$
\eta_{t}(u) \in B_{r} \cap\{I \leq 0\} \quad \forall t \geq 0,
$$

por (I.1). Por outro lado de (1.56), $b_{n, r}<0$ e $(P S)$ é satisfeito em $B_{r}$ por (I.3); pode-se usar a Teoria de Lusternik- Schnirelman [19], para encontrar infinitos pontos críticos de $I$ em $B_{r}$ tal que $I(u)<0$. Isto prova o item (1) do Teorema 1.4.

2. Agora consideramos $p<\frac{N+2}{N-2}$. Adaptaremos os argumentos do [3] para fazer a demonstração. Primeiro, note que (I.1) não é o mesmo que $I_{1}$ de [3]. Mais ainda, seja

$$
\hat{A}_{0}=B_{r} \cup\{I \geq 0\},
$$

claramente, temos que $H_{n} \cap \hat{A}_{0}$ é limitada para todo $n \in N$, isto é $\left(I_{5}\right)$ de [3] é satisfeita. Logo temos que

$$
\begin{aligned}
\Gamma^{*} & =\left\{h \in C(H, H): h \text { é um homeomorfismo ímpar e } h\left(B_{1}\right) \subset \hat{A}_{0}\right\}, \\
\Gamma_{n} & =\left\{K \in \Sigma: \gamma\left(K \cap h\left(\partial B_{1}\right)\right) \geq n, \forall h \in \Gamma^{*}\right\}
\end{aligned}
$$

e

$$
c_{n}=\inf _{K \in \Gamma_{n}} \max _{u \in K} I(u) .
$$

Note que o Lema 2.7 de [3] é satisfeita, escolhendo

$$
h(u)=r u,
$$

$r$ definido em $(I .1-I .3)$, temos que $h \in \Gamma^{*}$. Então encontramos novamente que $K \cap B_{r} \neq \emptyset, \forall K \in$ $\Gamma_{n}$, e segue que

$$
c_{n} \geq a>0 .
$$

Mais ainda, seja $\phi=\eta_{1}$, assim obtemos

$$
\phi^{-1}\left(\hat{A}_{0}\right) \subset \hat{A}_{0}
$$

Para mostrar este fato, é suficiente tomar $u \in B_{r}$, por outro lado, se $I(u)>0 \operatorname{logo} I\left(\phi^{-1}(u)\right)>I(u)$ e (1.57) é satisfeita trivialmente. Agora, se $u \in B_{r}$ e $w:=\phi^{-1} \notin B_{r}$, logo existe $\tau<1$ tal que $\eta_{\tau}(w) \in \partial B_{r}$. Por $(I .1)$ encontramos que $I\left(\eta_{\tau}(w)\right) \geq a>0$. Então $I(w) \geq I\left(\eta_{\tau}(w)\right)>0$ e $w \in \hat{A}_{0}$, provando (1.57).

Finalmente, como foi feito para $J_{\lambda}$, veja o Lema $1.9(i), I$ também satisfaz $(P S)$. Logo aplicando o Teorema 2.8 do artigo [3], temos a existência de infinitos pontos críticos de $I$ tal que $I(u)>0$. 


\section{Capítulo}

\section{Multiplicidade de Soluções}

Estudaremos agora a existência, não-existência e multiplicidade de soluções para a família de problemas

$$
\left(\tilde{P}_{\lambda}\right)\left\{\begin{aligned}
-\Delta u & =f_{\lambda}(x, u), & & x \in \Omega, \\
u & >0, & & x \in \Omega, \\
u & =0, & & x \in \partial \Omega,
\end{aligned}\right.
$$

onde $\Omega$ é um domínio limitado em $\mathbb{R}^{N}, N \geq 3$ e $\lambda>0$ um parâmetro. Um importante fato desta família é a dependência monótona sobre $\lambda$, isto é

$$
f_{\lambda}(x, s) \leq f_{\lambda^{\prime}}(x, s) \quad \text { se } \quad \lambda<\lambda^{\prime}
$$

Nossa hipótese geral sobre a família $f_{\lambda}(x, s)$ é

(H) Para cada $\lambda>0, f_{\lambda}: \Omega \times[0, \infty) \rightarrow \mathbb{R}$ é uma função de Carathéodory, com a propriedade de que para qualquer $s_{0}>0$, existe uma constante $A>0$, tal que

$$
\left|f_{\lambda}(x, s)\right| \leq A
$$

em quase todo ponto de $\Omega$, e todo $s \in\left[0, s_{0}\right]$. Além disso, se $\lambda<\lambda^{\prime}$, então $f_{\lambda}(x, s) \leq f_{\lambda^{\prime}}(x, s)$ em quase todo ponto de $\Omega$ e todo $s \geq 0$.

A seguinte hipótese trata acerca do comportamento de $f_{\lambda}(x, s)$ perto de $s=0$; isto implica $f_{\lambda}(x, 0) \geq$ 0 e, como assumimos $(H)$, vamos supor em todo o trabalho que:

$\left(H_{0}\right)$ Para cada $\lambda>0$ e cada $s_{0}>0$, existe $B>0$ tal que

$$
f_{\lambda}(x, s) \geq-B s,
$$

para quase todo ponto de $\Omega$ e todo $s \in\left[0, s_{0}\right]$.

Vamos sempre considerar que $f_{\lambda}(x, s)$ possa ser estendido para $s<0$, escrevendo $f_{\lambda}(x, s)=f_{\lambda}(x, 0)$, para $\lambda>$ 0 em quase todo ponto de $\Omega$ e $s<0$. 
Observamos que se $u \in H \cap L^{\infty}(\Omega)$ satisfaz a equação $-\Delta u=f_{\lambda}(x, u)$ em $H$, então pela hipótese $(H)$ e a teoria de regularidade (ver Apêndice A.1) implicam que $u \in W^{2, r}(\Omega)$ para qualquer $r<\infty$, e assim $u \in C^{1}(\bar{\Omega})$. Mais ainda, $u \geq 0$ (demonstrado mais na frente); e assim, temos $u>0$ em $\Omega$, e $\frac{\partial u}{\partial \nu}<0$ sobre $\partial \Omega$ se $u \neq 0$ (isto segue de $\left(H_{0}\right)$ e o Princípio do Máximo forte). Aqui $\nu$ denota o vetor normal exterior unitário.

Observe também que o funcional associado é

$$
I_{\lambda}(u):=\frac{1}{2} \int_{\Omega}|\nabla u|^{2}-\int_{\Omega} F_{\lambda}(x, u) d x
$$

onde $F_{\lambda}(x, u):=\int_{0}^{u} f_{\lambda}(x, t) d t$, está bem definido para $u \in H \cap L^{\infty}(\Omega)$.

As duas próximas hipóteses, serão usadas no nosso primeiro resultado.

$\left(H_{e}\right)$ Existe $\lambda>0$ e a função $g$ não decrescente, $\operatorname{com} \inf \left\{\frac{g(s)}{s}, s>0\right\}<\frac{1}{\|e\|_{\infty}}$, tal que

$$
f_{\lambda}(x, s) \leq g(s)
$$

em quase todo $x \in \Omega$ e todo $s \geq 0$; onde $e$ é solução de

$$
\left\{\begin{aligned}
-\Delta e & =1, & & x \in \Omega, \\
e & =0, & & x \in \partial \Omega,
\end{aligned}\right.
$$

e $\|\cdot\|_{\infty}$ denota a norma $L^{\infty}$.

$\left(H_{\Omega_{1}}\right)$ Para qualquer $\lambda>0$, existe um subdomínio suave $\Omega_{1}, s_{1}>0$, e $\theta_{1}>\lambda_{1}\left(\Omega_{1}\right)$ tal que

$$
f_{\lambda}(x, s) \geq \theta_{1} s,
$$

para quase todo ponto de $\Omega_{1}$ e todo $s \in\left[0, s_{1}\right]$; aqui $\lambda_{1}\left(\Omega_{1}\right)$ denota o primeiro autovalor de $-\Delta$ sobre $H_{0}^{1}\left(\Omega_{1}\right)$.

Alguns comentários sobre as duas últimas hipóteses.

- A hipótese $\left(H_{e}\right)$ é mais uma condição para garantir a existência de uma supersolução. Está condição é motivada pelo fato que uma supersolução para uma equação do tipo $-\Delta u=f(u)$ pode ser obtida se tivermos uma supersolução dada por uma outra equação da forma $-\Delta u=g(u)$, com $f(s) \leq g(s) \forall s$.

- A hipótese $\left(H_{\Omega_{1}}\right)$ é condição de sublinearidade local no 0, a que está satisfeita, por exemplo, se a seguinte condição mais forte ocorre

$$
\lim _{\substack{s \rightarrow 0 \\ s>0}} \frac{f_{\lambda}(x, s)}{s}=\infty, \quad \text { uniformemente para } x \in \Omega_{1} .
$$

A hipótese $\left(H_{\Omega_{1}}\right)$ é usada para construir a subsolução.

Teorema 2.1 (Existência de uma solução sem condição de crescimento). Sobre as hipóteses $(H),\left(H_{0}\right),\left(H_{e}\right)$ $e\left(H_{\Omega_{1}}\right)$, existe $0<\Lambda \leq \infty$ tal que o problema $\left(\tilde{P}_{\lambda}\right)$ tem ao menos uma solução u com $I_{\lambda}(u)<0$ para $0<\lambda<\Lambda$, e nenhuma solução para $\lambda>\Lambda$. 
Observação 2.1. Note que, na presente generalidade $\Lambda$ pode ser $\infty$. Um exemplo é fornecido pela família como acima tal que para cada $\lambda>0$, existe $M_{\lambda}>0$ com $f_{\lambda}\left(x, M_{\lambda}\right)<0$ em quase todo ponto de $\Omega$. Neste caso, a constante $M_{\lambda}$ é uma supersolução.

Teorema 2.2 (Não existência de soluções para $\lambda$ grande). Sob as hipóteses $(H),\left(H_{0}\right),\left(H_{e}\right),\left(H_{\Omega_{1}}\right) e\left(H_{\tilde{\Omega}}\right)$, onde

$\left(H_{\tilde{\Omega}}\right)$ Existe a função $h$ com $h(\lambda) \rightarrow \infty$ quando $\lambda \rightarrow \infty$, um subdomínio suave $\tilde{\Omega}$ e $\tilde{m} \in L^{\infty}(\Omega)$ com $\tilde{m} \geq 0, \tilde{m} \not \equiv 0$, tal que

$$
f_{\lambda}(x, s) \geq h(\lambda) \tilde{m}(x) s
$$

para todo $\lambda>0$ em quase todo ponto de $\Omega$ e para todo $s \geq 0$.

Então $\Lambda<\infty$.

Observação 2.2. A hipótese $\left(H_{\tilde{\Omega}}\right)$ pode ser vista como uma versão localizada da condição suficiente de não existência para

$$
\left\{\begin{aligned}
-\Delta u & =l(u), & & x \in \Omega, \\
u & >0, & & x \in \Omega, \\
u & =0, & & x \in \partial \Omega,
\end{aligned}\right.
$$

onde $\inf \left\{\frac{l(s)}{s}, s \geq 0\right\}>\lambda_{1}(\Omega)$.

Devido à ausência da condição de crescimento, temos até agora definida uma solução como a função em $H \cap L^{\infty}(\Omega)$. Embora, se a seguinte condição de crescimento com respeito a $s$ na não-linearidade de $f_{\lambda}(x, s)$ é assumida, então podemos falar de uma $H$ solução no sentido usual:

(G) Para qualquer $[r, R] \subset\{\lambda>0\}$, existem $d_{1}$, $d_{2}$ e $\sigma \leq 2^{*}-1$ tal que

$$
\left|f_{\lambda}(x, s)\right| \leq d_{1}+d_{2} s^{\sigma}
$$

para todo $\lambda \in[r, R]$, e quase todo ponto em $\Omega$ e todo $s \geq 0$.

Observação 2.3. Se $\sigma<2^{*}-1$ em $(G)$, então qualquer $u \in H$, é solução de $-\Delta u=f_{\lambda}(x, u)$ que pertence a $W^{2, r}(\Omega)$ para qualquer $r<\infty$ e consequentemente a $C^{1}(\bar{\Omega})$. Está conclusão também é satisfeita se $\sigma$ em $(G)$ é igual a $2^{*}-1$.

Condição $(G)$ com $\sigma \leq 2^{*}-1$, também implica, que o funcional $I_{\lambda}(u)$ está bem definido para $u \in H$.

Nosso objetivo agora é provar a existência de uma solução para $\lambda=\Lambda$, podemos supor a seguinte condição:

$(A R)_{d}$ Para qualquer $[r, R] \subset\{\lambda>0\}$, existe $\theta>2, \rho<2, d \geq 0$ e $s_{0} \geq 0$, tal que

$$
\theta F_{\lambda}(x, s) \leq s f_{\lambda}(x, s)+d s^{\rho}
$$

para todo $\lambda \subset[r, R]$, quase todo ponto em $\Omega$ e todo $s \geq s_{0}$.

Observação 2.4. Esta condição $(A R)_{d}$ é uma versão mais fraca da condição clássica de superquadraticidade de Ambrosetti-Rabinowitz. 
Teorema 2.3. (Existência de uma solução para $\lambda=\Lambda$ ). Sob as hipóteses do Teorema (2.2), e além de $(G),(A R)_{d}$ e a continuidade de $f_{\lambda}(x, s)$ com respeito a $\lambda$ (para quase todo ponto em $\Omega$, e uniformemente limitado para $s)$. Então o problema $\left(\tilde{P}_{\lambda}\right)$ tem ao menos uma solução $u$, com $I_{\lambda}(u) \leq 0$ para $\lambda=\Lambda$.

Observação 2.5. A uniformidade com respeito a $\lambda \in[r, R]$ em $(G)$ e $(A R)_{d}$ é usado somente no Teorema (2.3) para tratar como o caso $\lambda=\Lambda$. Não é necessário nos seguintes teoremas $(2.4)-(2.6)$, onde $\lambda<\Lambda$ poderia ser fixo.

Agora discutiremos a multiplicidade de soluções para famílias subcríticas, isto é, as que satisfazem $(G)$ como $\sigma<2^{*}-1$. Nosso objetivo, é mostrar a existência de ao menos duas soluções quando $\lambda<\Lambda$. Para isso, temos que fortalecer um pouco algumas das hipóteses do Teorema (2.1). A condição $\left(H_{0}\right)$ será representada por

$\left(H_{0}\right)^{\prime}$ Para qualquer $\lambda>0$ e todo $s_{0}>0$, existe $B \geq 0$, tal que em quase todo ponto de $\Omega$,

$$
s \mapsto f_{\lambda}(x, s)+B s
$$

é não-decrescente sob $\left[0, s_{0}\right]$, mais ainda $f_{\lambda}(x, 0) \geq 0$ para todo $\lambda>0$ em quase todo ponto de $\Omega$.

Observação 2.6. A condição $\left(H_{0}\right)^{\prime}$ é um requisito clássico, quando tratamos com sub e supersoluções.

A monotonicidade da família $f_{\lambda}$ é também assumida e pode a ser estrita no seguinte sentido:

(M) Para qualquer $\lambda<\lambda^{\prime}$ e todo $u \in C_{0}^{1}(\bar{\Omega}) \operatorname{com} u>0$ em $\Omega$

$$
f_{\lambda}(x, u(x)) \supsetneqq f_{\lambda^{\prime}}(x, u(x)) .
$$

Também podemos supor que:

$\left(H_{\Omega_{2}}\right)$ Para todo $\lambda>0$, existe um subdomínio $\Omega_{2}, s_{2}$ e $\theta_{2}>0$ tal que

$$
F_{\lambda}(x, s) \geq \theta_{2} s^{2}
$$

em quase todo ponto de $\Omega_{2}$ e todo $s \geq s_{2}$.

Observação 2.7. A condição $\left(H_{\Omega_{2}}\right)$ é implicada por uma condição de superlinearidade local no $\infty$, da forma

$$
\lim _{s \rightarrow \infty} \frac{f_{\lambda}(x, s)}{s}=\infty
$$

uniformemente para $x \in \Omega_{2}$. Isto é utilizado junto com $(A R)_{d}$ para verificarmos a geometria do Teorema do Passo da Montanha.

Teorema 2.4. (Existência de uma segunda solução no caso subcrítico). Além das hipóteses do Teorema $(2.1)$, suponha $(G)$ como $\sigma<2^{*}-1,(A R)_{d},\left(H_{0}\right)^{\prime},(M)$ e $\left(H_{\Omega_{2}}\right)$. Então o problema $\left(\tilde{P}_{\lambda}\right)$ tem ao menos duas soluções $u, v$ para $0<\lambda<\Lambda$, com $u<v$ em $\Omega, \frac{\partial u}{\partial \nu}>\frac{\partial v}{\partial \nu}$ sobre $\partial \Omega$ e $I_{\lambda}(u)<0$.

Finalmente, consideramos multiplicidade para as famílias críticas. Isto é, que $f_{\lambda}(x, s)$ comporta-se no infinito como $b(x) s^{p} \operatorname{com} p=2^{*}-1$. Então escrevemos a função $f_{\lambda} \operatorname{como}$

$$
f_{\lambda}(x, s)=h_{\lambda}(x, s)+b(x) s^{p}
$$


onde distinguimos dois casos:

i. $h_{\lambda}$ satisfaz $(G) \operatorname{com} \sigma<1, b(x)$ pode mudar de sinal;

ii. $h_{\lambda} \operatorname{satisfaz}(G) \operatorname{com} \sigma<2^{*}-1, b(x) \geq 0$ em $\Omega$.

Estudaremos primeiro o caso $(i)$.

Teorema 2.5. (Existência de uma segunda solução no caso crítico com $\sigma<1$ ). Além das hipóteses do Teorema (2.1), suponha que $f_{\lambda}(x, s)$ satisfaz $\left(H_{0}\right)^{\prime}$ e $(M)$. Suponha também que $f_{\lambda}(x, s)$ pode-se escrever como em (2.1) com $p=2^{*}-1, h_{\lambda}(x, s)$ satisfazendo $(G)$ com $\sigma<1$, e $h_{\lambda}(x, s)$ não decrescente em relação a s para qualquer $\lambda>0$ em quase todo ponto de $\Omega$. Suponha também que, b(x) em (2.1) não é identicamente nula, com $b \in L^{\infty}(\Omega)$ e satisfazendo

(b) para algum $x_{0} \in \Omega$, alguma bola $B_{1} \subset \Omega$ em torno de $x_{0}$, alguma constante $M$ e algum $\gamma$ com $\gamma>2^{*}$ quando $N \geq 5, \gamma \geq 2^{*}$ quando $N=4$ e $\gamma>3 / 5$ quando $N=3$, têm-se

$$
0 \leq\|b\|_{\infty}-b(x) \leq M\left|x-x_{0}\right|^{\gamma}
$$

em quase todo ponto de $B_{1}$.

Então o problema $\left(\tilde{P}_{\lambda}\right)$ tem ao menos duas soluções $u, v$ para $0<\lambda<\Lambda$, com $u<v$ em $\Omega, \frac{\partial u}{\partial \nu}>$ $\frac{\partial v}{\partial \nu}$ sobre $\partial \Omega$ e $I_{\lambda}(u)<0$.

Observação 2.8. A hipótese (b) implica $\left\|b^{-}\right\|_{\infty} \leq\left\|b^{+}\right\|_{\infty}$, com algumas limitações sobre a forma em que $b(x)$ se aproxima de $\|b\|_{\infty}$. Isso é trivial se $b(x)=\|b\|_{\infty}$ em quase todo ponto de uma bola pequena.

Agora, estudaremos o caso crítico $(i i)$.

Teorema 2.6. (Existência de uma segunda solução no caso crítico com $\sigma<2^{*}-1$ ). Em adição das hipóteses do Teorema (2.1) suponha que $f_{\lambda}(x, s)$ satisfaz $\left(H_{0}\right)^{\prime}$ e $(M)$. Suponha também que $f_{\lambda}(x, s)$ pode-se escrever como em (2.1), com $p=2^{*}-1, h_{\lambda}(x, s)$ satisfaz $(G) \operatorname{com} \sigma<2^{*}-1, h_{\lambda}(x, s)$ não decrescente com respeito a $s$, para qualquer $\lambda>0$ em quase todo ponto de $\Omega$, e $h_{\lambda}(x, s)$ satisfaz $(A R)_{d}$. Suponha ainda que b em (2.1) é não negativa e não é identicamente nula em $\Omega$, pertencendo a $L^{\infty}(\Omega)$ e satisfaz a condição (b) acima. Então o problema $\left(\tilde{P}_{\lambda}\right)$ tem ao menos duas soluções $u$, v para $0<\lambda<\Lambda$, com $u<v$ em $\Omega, \frac{\partial u}{\partial \nu}>\frac{\partial v}{\partial \nu}$ sobre $\partial \Omega$ e $I_{\lambda}(u)<0$.

Observação 2.9. No Teorema $(2.6), h_{\lambda}(x, s)$ permite-se qualquer crescimento subcrítico, sob a hipótese $(A R)_{d} \operatorname{para} h_{\lambda}(x, s)$ e $b(x) \geq 0$.

\subsection{Existência de uma solução sem condição de crescimento}

Nesta seção mostraremos o Teorema 2.1.

Demonstração. Começamos provando a existência de uma supersolução de $\left(\tilde{P}_{\lambda}\right)$ para o valor de $\lambda$ dado pela hipótese $\left(H_{e}\right)$. Denotaremos por $e$ a solução do problema

$$
\left\{\begin{aligned}
-\Delta e & =1, & & x \in \Omega, \\
e & =0, & & x \in \partial \Omega .
\end{aligned}\right.
$$


Tomando $\lambda$ e $g$ da hipótese $\left(H_{e}\right)$, existe $M>0$ tal que $\frac{1}{\|e\|_{\infty}} \geq \frac{g\left(M\|e\|_{\infty}\right)}{M\|e\|_{\infty}}$, então $M \geq$ $g\left(M\|e\|_{\infty}\right)$ assim temos,

$$
-\Delta(M e)=M(-\Delta e)=M \geq g\left(M\|e\|_{\infty}\right) \geq g(M e) \geq f_{\lambda}(x, M e) .
$$

Isto mostra que $M e$ é uma supersolução clássica de $\left(\tilde{P}_{\lambda}\right)$.

Agora, construiremos uma subsolução de $\left(\tilde{P}_{\lambda}\right)$ utilizando o subdomínio $\Omega_{1}$, dada pela hipótese $\left(H_{\Omega_{1}}\right)$. Seja $\varphi_{1}>0$ a autofunção associada ao primeiro autovalor $\lambda_{1}\left(\Omega_{1}\right)$ do problema

$$
(P A) \quad\left\{\begin{aligned}
-\Delta u & =\lambda u, & & x \in \Omega_{1}, \\
u & =0, & & x \in \partial \Omega_{1},
\end{aligned}\right.
$$

onde $\Omega_{1}$ é o subdomínio dado pela hipótese $\left(H_{\Omega_{1}}\right)$.

A regularidade de $\varphi_{1}$ nos permite estendê-la para todo $\Omega$ fazendo

$$
u(x)=\left\{\begin{aligned}
\varphi_{1}(x), & x \in \Omega_{1}, \\
0, & x \in \Omega \backslash \Omega_{1},
\end{aligned}\right.
$$

de modo que $u \in H \cap L^{\infty}(\Omega)$.

Vamos denotar $u_{\varepsilon}=\varepsilon u$ para $\varepsilon>0$. Como $\partial \Omega$ é de classe $C^{1}$, dado $v \in C_{0}^{\infty}(\Omega)$, com $v \geq 0$, vale

$$
\int_{\Omega} \nabla u_{\varepsilon} \nabla v d x=\int_{\partial \Omega} \frac{\partial u_{\varepsilon}}{\partial \eta} v d x-\int_{\Omega}\left(\Delta u_{\varepsilon}\right) v d x
$$

como o suporte de $v$ está contido em $\Omega$ e $u_{\epsilon}=0$ em $\Omega \backslash \Omega_{1}$ concluímos que

$$
\int_{\Omega} \nabla u_{\varepsilon} \nabla v d x=-\int_{\Omega_{1}}\left(\Delta u_{\varepsilon}\right) v d x
$$

Por $\left(H_{\Omega_{1}}\right)$, sabemos que existe $s_{1}>0$ tal que

$$
f_{\lambda}(x, s) \geq \theta_{1} s>\lambda_{1}\left(\Omega_{1}\right) s
$$

em quase todo ponto de $\Omega$, sempre que $0 \leq s \leq s_{1}$. Logo, tomando $\varepsilon>0$ tal que $\varepsilon\|u\|_{\infty} \leq s_{1}$, temos que

$$
f_{\lambda}\left(x, u_{\varepsilon}\right) \geq \theta_{1} u_{\varepsilon}>\lambda_{1}\left(\Omega_{1}\right) u_{\varepsilon} \quad \text { em quase todo ponto de } \Omega
$$

pois $0 \leq \varepsilon u \leq s_{1}$.

Além disso, como $\varphi_{1}$ é uma autofunção associada ao autovalor $\lambda_{1}$ do problema $(P A)$ e $v \geq 0$ segue que $\left(-\Delta u_{\varepsilon}\right) v=\lambda_{1}\left(\Omega_{1}\right) u_{\varepsilon} v$. Logo por $(2.2)$ e (2.3) temos

$$
\int_{\Omega} \nabla u_{\varepsilon} \nabla v d x=-\int_{\Omega_{1}}\left(\Delta u_{\varepsilon}\right) v d x=\int_{\Omega_{1}} \lambda_{1}\left(\Omega_{1}\right) u_{\varepsilon} v d x \leq \int_{\Omega_{1}} f_{\lambda}\left(x, u_{\epsilon}\right) v d x
$$


como $u_{\varepsilon}=0$ em $\Omega \backslash \Omega_{1}$ e $\left(H_{0}\right)$ nos garante que $f(x, 0) \geq 0$ então $\int_{\Omega_{\backslash} \Omega_{1}} f_{\lambda}\left(x, u_{\lambda}\right) v d x \geq 0$, e portanto

$$
\int_{\Omega_{1}} f_{\lambda}\left(x, u_{\varepsilon}\right) v d x \leq \int_{\Omega_{1}} f_{\lambda}\left(x, u_{\varepsilon}\right) v d x+\int_{\Omega \backslash \Omega_{1}} f_{\lambda}\left(x, u_{\varepsilon}\right) v d x=\int_{\Omega} f_{\lambda}\left(x, u_{\varepsilon}\right) v d x
$$

logo por (2.3) concluímos que,

$$
\int_{\Omega} \nabla u_{\varepsilon} \nabla v d x \leq \int_{\Omega} f_{\lambda}\left(x, u_{\varepsilon}\right) v d x
$$

e $\operatorname{assim} u_{\varepsilon}=\varepsilon u$ é uma subsolução fraca para o problema $\left(\tilde{P}_{\lambda}\right)$.

Como o suporte de $\varepsilon$ está contido em $\bar{\Omega}_{1}$ e além disso $\Omega_{1} \subset \subset \Omega$, a supersolução $M e$ atinge mínimo positivo em $\bar{\Omega}_{1}$. Portanto, se é necessário, podemos tomar $\varepsilon>0$ suficientemente pequeno, de tal forma que $\varepsilon\|u\| \leq M e(x), x \in \bar{\Omega}_{1}$.

Tomando $\underline{c}=-\varepsilon\|u\|_{\infty}$ e $\bar{c}=M\|e\|_{\infty}$, temos que

$$
\underline{c} \leq \varepsilon u \leq M e \leq \bar{c}, \quad x \in \Omega
$$

Logo, pelo Teorema de sub e supersolução (ver Apêndice $C$, Teorema C.2), o problema $\left(\tilde{P}_{\lambda}\right)$ admite uma solução fraca $u \in H \cap L^{\infty}(\Omega)$ que satisfaz $\varepsilon u \leq u \leq M e$, para o valor $\lambda$ dada na hipótese $\left(H_{e}\right)$.

Assim, prova-se que

$$
\Lambda=\sup \left\{\lambda>0,\left(\tilde{P}_{\lambda}\right) \text { tem uma solução }\right\}>0 .
$$

Faltaría mostra que para cada $0<\lambda<\Lambda,\left(\tilde{P}_{\lambda}\right)$ tem uma solução $u$ tal que $I_{\lambda}(u)<0$.

Seja $0<\lambda<\Lambda$, e tome $\bar{\lambda}$ tal que $\lambda<\bar{\lambda}<\Lambda$ e $\left(\tilde{P}_{\bar{\lambda}}\right)$ tem solução $\bar{u}$, isto é possível pela definição de $\Lambda$.

Como a família $f_{\lambda}$ é monótona, temos que

$$
-\Delta \bar{u}=f_{\bar{\lambda}}(x, \bar{u}) \geq f_{\lambda}(x, \bar{u})
$$

o que mostra que $\bar{u}$ é uma supersolução de $\left(\tilde{P}_{\lambda}\right)$.

Pelo feito anteriormente, usando o subdomínio $\Omega_{1}$ da hipótese $\left(H_{\Omega_{1}}\right)$ mostra que para $\varepsilon>0$ suficientemente pequeno, $\varepsilon \varphi_{1}$ é uma subsolução fraca de $\left(\tilde{P}_{\lambda}\right)$; a qual satisfaz que $\varepsilon \varphi_{1} \leq \bar{u}$ em $\Omega$.

Agora, pelo Teorema 2.4 de [22] segue que existe uma solução $u_{0} \in H \cap L^{\infty}(\Omega)$ de $\left(\tilde{P}_{\lambda}\right)$ a qual satisfaz

$$
I_{\lambda}\left(u_{0}\right)=\min \left\{I_{\lambda}(u) ; u \in H \text { e } \varepsilon \varphi_{1} \leq u \leq u \leq \bar{u}\right\}
$$

Por outro lado, por $\left(H_{\Omega_{1}}\right)$ temos para todo $\lambda>0$, existe um subdomínio $\Omega_{1}$ suave, $s_{1}>0$ e $\theta_{1}>\lambda_{1}\left(\Omega_{1}\right)$ tal que

$$
f_{\lambda}(x, s) \geq \theta_{1} s \quad \text { em quase todo ponto de } \Omega,
$$

e para todo $0 \leq s \leq s_{1}$.

Assim,

$$
\begin{gathered}
\int_{\Omega} \int_{0}^{u} f_{\lambda}(x, s) d s d x \geq \int_{\Omega} \int_{0}^{u} \theta_{1} s d s d x \geq 0 \\
\int_{\Omega} F_{\lambda}(x, u) d x \geq 0, \quad \forall u \in H .
\end{gathered}
$$


Logo,

$$
\begin{aligned}
I_{\lambda}\left(\varepsilon \varphi_{1}\right) & =\frac{\varepsilon^{2}}{2}\left\|\varphi_{1}\right\|^{2}-\int_{\Omega} F_{\lambda}\left(x, \varepsilon \varphi_{1}\right) d x \\
& =\frac{\varepsilon^{2}}{2} \int_{\Omega}\left|\nabla \varphi_{1}\right|^{2} d x-\int_{\Omega} F_{\lambda}\left(x, \varepsilon \varphi_{1}\right) d x .
\end{aligned}
$$

Assim, sendo

$$
\begin{aligned}
I_{\lambda}\left(u_{0}\right) & =\min \left\{I_{\lambda}(u) ; u \in H \quad \text { e } \quad \varepsilon \varphi_{1} \leq u \leq \bar{u}\right\} \\
& \leq I_{\lambda}(u), u \in H \quad \text { e } \quad \varepsilon \varphi_{1} \leq u \leq \bar{u}
\end{aligned}
$$

teríamos que

$$
I_{\lambda}\left(u_{0}\right) \leq I_{\lambda}\left(\varepsilon \varphi_{1}\right)=\frac{\varepsilon^{2}}{2} \int_{\Omega}\left|\nabla \varphi_{1}\right|^{2} d x-\int_{\Omega} F_{\lambda}\left(x, \varepsilon \varphi_{1}\right) d x,
$$

para $\varepsilon$ suficientemente pequeno (tal que $\varepsilon \varphi_{1} \leq s_{1}$ ). Portanto temos que $I_{\lambda}\left(u_{0}\right)<0$. Isto completa a prova do Teorema 2.1.

\subsection{Não existência de soluções para $\lambda$ grande}

Nesta seção mostraremos o Teorema 2.2 .

Demonstração. Deve-se mostrar que para $\lambda$ suficientemente grande o problema $\left(\tilde{P}_{\lambda}\right)$ não tem solução. Suponha por contradição que o problema $\left(\tilde{P}_{\lambda}\right)$ tem uma solução $u \in H \cap L^{\infty}(\Omega)$.

Seja agora, $\tilde{\varphi}>0$ a autofunção associada ao primeiro autovalor $\lambda_{1}(\tilde{m}, \tilde{\Omega})$ do problema de autovalor com peso $\tilde{m}$.

$$
(\overline{P A}) \quad\left\{\begin{aligned}
-\Delta u & =\lambda \tilde{m}(x) u, & & x \in \tilde{\Omega}, \\
u & =0, & & x \in \partial \tilde{\Omega},
\end{aligned}\right.
$$

onde $\tilde{\Omega}$ é um subdomínio dado pela hipótese $\left(H_{\tilde{\Omega}}\right)$.

A regularidade de $\tilde{\varphi}$ nos permite estendê-lo para todo $\Omega$ fazendo

$$
u(x)=\left\{\begin{aligned}
\tilde{\varphi}(x), & x \in \tilde{\Omega}, \\
0, & x \in \Omega \backslash \tilde{\Omega},
\end{aligned}\right.
$$

de modo que $u \in H \cap L^{\infty}(\Omega)$. Então temos que,

$$
\begin{aligned}
\int_{\Omega} \nabla u \nabla \varphi d x & =\int_{\partial \tilde{\Omega}} u \frac{\partial \tilde{\varphi}}{\partial \nu} d x+\int_{\tilde{\Omega}} u(-\Delta \tilde{\varphi}) d x \\
& =\int_{\tilde{\Omega}} u(-\Delta \tilde{\varphi}) d x \\
& \leq \int_{\tilde{\Omega}} u\left(\lambda_{1}(\tilde{m}, \tilde{\Omega}) \tilde{\varphi} \tilde{m}\right) d x=\lambda_{1}(\tilde{m}, \tilde{\Omega}) \int_{\tilde{\Omega}} \tilde{m} u \tilde{\varphi} d x .
\end{aligned}
$$

Por outro lado, pela hipótese $\left(H_{\tilde{\Omega}}\right)$ temos

$$
\int_{\Omega} \nabla u \nabla \tilde{\varphi} d x=\int_{\Omega} f_{\lambda}(x, u) \tilde{\varphi} d x \geq h(\lambda) \int_{\tilde{\Omega}} \tilde{m} u \tilde{\varphi} d x .
$$


Como $\int_{\tilde{\Omega}} \tilde{m} u \tilde{\varphi} d x>0$, e de $(2.9)$ e $(2.10)$ temos que

$$
h(\lambda) \int_{\tilde{\Omega}} \tilde{m} u \tilde{\varphi} d x \leq \int_{\Omega} \nabla u \nabla \tilde{\varphi} d x \leq \lambda_{1}(\tilde{m}, \tilde{\Omega}) \int_{\tilde{\Omega}} \tilde{m} u \tilde{\varphi} d x
$$

assim, $h(\lambda) \leq \lambda_{1}(\tilde{m}, \tilde{\Omega})$. O que é uma contradição, pois $h(\lambda) \rightarrow+\infty$, quando $\lambda \rightarrow+\infty$. Isto completa a prova do Teorema 2.2 .

\subsection{Existência de uma solução para $\lambda=\Lambda$}

Nesta seção mostraremos o Teorema 2.3.

Demonstração. A continuidade de $f_{\lambda}$ com respeito a $\lambda$, bem como as hipóteses $(G)$ e $(A R)_{d}$ que são satisfeitas uniformemente para todo $\lambda \in[r, R]$, serão utilizadas aqui.

Seja $\lambda_{k} \rightarrow \Lambda$ com $0<\lambda_{k}<\Lambda$, e $\lambda_{k}$ crescente; e seja $u_{k}$ a solução do problema $\left(\tilde{P}_{\lambda_{k}}\right) \operatorname{com} I\left(u_{k}\right)<0$.

Primeiro mostraremos que a sequência $\left(u_{k}\right)$ é limitada em $H$.

Afirmação 2.1. $\left(u_{k}\right)$ é limitada em $H$.

De fato, como

$$
I\left(u_{k}\right)=I_{\lambda_{k}}\left(u_{k}\right)=\frac{1}{2} \int_{\Omega}\left|\nabla u_{k}\right|^{2} d x-\int_{\Omega} F_{\lambda_{k}}\left(x, u_{k}\right) d x<0
$$

assim,

$$
\frac{1}{2} \int_{\Omega}\left|\nabla u_{k}\right|^{2} d x<\int_{\Omega} F_{\lambda_{k}}\left(x, u_{k}\right) d x
$$

Pela hipótese $(A R)_{d}$, temos

$$
\frac{\theta}{2} \int_{\Omega}\left|\nabla u_{k}\right|^{2} d x<\int_{\Omega}\left(u_{k} f_{\lambda_{k}}\left(x, u_{k}\right)+d u_{k}^{\rho}\right) d x+C_{1},
$$

assim

$$
\frac{\theta}{2}\left\|u_{k}\right\|^{2}-\int_{\Omega} u_{k} f_{\lambda_{k}}\left(x, u_{k}\right) d x \leq d \int_{\Omega} u_{k}^{\rho} d x+C_{1},
$$

para alguma constante positiva $C_{1}$.

Mas $\int_{\Omega} u_{k} f_{\lambda_{k}}\left(x, u_{k}\right) d x=\left\|u_{k}\right\|^{2}$ pois de fato, como $u_{k}$ é solução de $\left(\tilde{P}_{\lambda_{k}}\right)$, segue que, $u_{k}$ é um ponto crítico de $I_{\lambda_{k}}$, assim $I_{\lambda_{k}}^{\prime}\left(u_{k}\right) u_{k}=0, \operatorname{logo}\left\|u_{k}\right\|^{2}-\int_{\Omega} f_{\lambda}\left(x, u_{k}\right) u_{k} d x=0$. E consequentemente de $(2.12)$ temos,

$$
\begin{gathered}
\frac{\theta}{2}\left\|u_{k}\right\|^{2}-\left\|u_{k}\right\|^{2} \leq d \int_{\Omega} u_{k}^{\rho} d x+C_{1} \\
\left(\frac{\theta}{2}-1\right)\left\|u_{k}\right\|^{2} \leq C_{2}\left\|u_{k}\right\|^{\rho}+C_{1}
\end{gathered}
$$

para alguma constante positiva $C_{2}$.

Como $\rho<2$, segue que $\left\|u_{k}\right\|^{\rho} \leq\left\|u_{k}\right\|^{2}$, assim

$$
\begin{aligned}
{\left[\left(\frac{\theta}{2}-1\right)-C_{2}\right]\left\|u_{k}\right\|^{2} } & \leq C_{1} \\
\left\|u_{k}\right\|^{2} & \leq \frac{C_{1}}{\left[\left(\frac{\theta}{2}-1\right)-C_{2}\right]}
\end{aligned}
$$


agora, como $\theta>2$ segue que $\left(u_{k}\right)$ é limitada em $H$.

Fazendo uso da limitação em $(G)$, note que em particular para subsequências $u_{k} \rightarrow u$ em $H \cap C(\bar{\Omega})$. Para $\sigma<2^{*}-1$, é de modo direto. Ver [4]. Para $\sigma=2^{*}-1$, ver [7].

É verdade que $u$ é solução de $-\Delta u=f_{\Lambda}(x, u)$ em $\Omega$ e $u \geq 0$ em $\Omega$ e $u=0$ sobre $\partial \Omega$, e também temos que $I_{\Lambda}(u) \leq 0$.

Somente resta-nos mostrar que $u$ não é identicamente nula.

Afirmação 2.2. u não é identicamente nula.

Suponha por contradição que $u \equiv 0$.

Utilizaremos a hipótese $\left(H_{\Omega_{1}}\right)$ para $\lambda=\lambda_{1}$, o primeiro elemento da sequência crescente $\lambda_{k}$. Considere $\left(u_{k}\right) \subset H$ tal que $u_{k} \rightarrow 0$.

Seja como antes, $\Omega_{1}$ o subdomínio correspondente e $\varphi_{1}>0$ a autonfunção associada ao primeiro autovalor $\lambda_{1}\left(\Omega_{1}\right)$ de $-\Delta$ sobre $H_{0}^{1}\left(\Omega_{1}\right)$. Temos

$$
\int_{\Omega} \nabla u_{k} \nabla \varphi_{1} d x=\int_{\Omega_{1}} f_{\lambda_{k}}\left(x, u_{k}\right) \varphi_{1} d x \geq \int_{\Omega_{1}} f_{\lambda_{1}}\left(x, u_{k}\right) \varphi_{1} d x \geq \theta_{1} \int_{\Omega_{1}} u_{k} \varphi_{1} d x
$$

para $k$ suficientemente grande $\left(\operatorname{assim} 0 \leq u_{k} \leq s_{1}\right.$ para $x \in \Omega_{1}$, o que é possível desde que $u_{k} \rightarrow 0$ uniformemente em $\bar{\Omega}$ ). Por outro lado,

$$
\int_{\Omega_{1}} \nabla u_{k} \nabla \varphi_{1} d x=\int_{\partial \Omega_{1}} u_{k} \frac{\partial \varphi_{1}}{\partial \nu} d x+\int_{\Omega_{1}} u_{k}\left(-\Delta \varphi_{1}\right) d x \leq \lambda_{1}\left(\Omega_{1}\right) \int_{\Omega_{1}} u_{k} \varphi_{1} d x
$$

assim, como $\int_{\Omega_{1}} u_{k} \varphi_{1} d x>0$ e de (2.13) combinado com (2.14) segue que $\theta_{1} \leq \lambda_{1}\left(\Omega_{1}\right)$. O que é uma contradição, pois $\theta_{1}>\lambda_{1}\left(\Omega_{1}\right)$. Isto completa a prova do Teorema (2.3).

\subsection{Existência de uma segunda solução no caso subcrítico}

Nesta seção mostraremos o Teorema 2.4.

Demonstração. Deve-se mostrar a existência de uma segunda solução de $\left(\tilde{P}_{\lambda}\right)$ para cada $0<\lambda<\Lambda$. Fixe $\lambda$, introduzimos $\underline{u}, \bar{u}$ e considerando a solução $u_{0}$ de $\left(\tilde{P}_{\lambda}\right)$ construída no Teorema $(2.1)$.

Afirmação 2.3. As seguintes desigualdades são verdadeiras

$$
\begin{gathered}
\underline{u}<u_{0}<\bar{u}, \quad x \in \Omega \\
\frac{\partial \underline{u}}{\partial \nu}>\frac{\partial u_{0}}{\partial \nu}>\frac{\partial \bar{u}}{\partial \nu}, \quad x \in \partial \Omega
\end{gathered}
$$

onde $\underline{u}:=\varepsilon \varphi_{1}$, com $\varphi_{1}$ primeira autofunção de $(-\Delta)$ em $H_{0}^{1}\left(\Omega_{1}\right)$.

De fato, seja $\varphi_{1}>0$ a autonfunção associada ao primeiro autovalor $\lambda_{1}\left(\Omega_{1}\right)$ do problema

$$
(P A) \quad\left\{\begin{aligned}
-\Delta u & =\lambda u, & & x \in \Omega_{1} \\
u & =0, & & x \in \partial \Omega_{1}
\end{aligned}\right.
$$


onde $\Omega_{1}$ é um subdomínio dado pela hipótese $\left(H_{\Omega_{1}}\right)$ e como $\underline{u}=\varepsilon \varphi_{1}$ é subsolução, temos que

$$
\left\{\begin{aligned}
-\Delta \underline{u} & \leq f_{\lambda}(x, \underline{u}), & & x \in \Omega_{1}, \\
\underline{u} & \leq 0, & & x \in \partial \Omega_{1} .
\end{aligned}\right.
$$

Por outro lado, como $u_{0}$ é solução de $\left(\tilde{P}_{\lambda}\right)$ temos que

$$
\left\{\begin{aligned}
-\Delta u_{0} & =f_{\lambda}\left(x, u_{0}\right), & & x \in \Omega, \\
u_{0} & =0, & & x \in \partial \Omega .
\end{aligned}\right.
$$

Pela regularidade de $\varphi_{1}$ permite estender $\underline{u}$ para todo $\Omega$ fazendo

$$
\underline{u}(x)= \begin{cases}\varepsilon \varphi_{1}(x), & x \in \Omega_{1}, \\ 0, & x \in \Omega \backslash \Omega_{1},\end{cases}
$$

assim temos que

$$
\left\{\begin{aligned}
-\Delta \underline{u} & \leq f_{\lambda}(x, \underline{u}), & & x \in \Omega, \\
\underline{u} & \leq 0, & & x \in \partial \Omega .
\end{aligned}\right.
$$

É claro que

$$
\begin{gathered}
\underline{u}<u_{0}<\bar{u}, \quad x \in \Omega \backslash \Omega_{1}, \\
\frac{\partial \underline{u}}{\partial \nu}>\frac{\partial u_{0}}{\partial \nu}>\frac{\partial \bar{u}}{\partial \nu}, \quad x \in \partial \Omega \backslash \partial \Omega_{1} .
\end{gathered}
$$

Por outro lado, $\underline{u} \not \equiv u_{0}$ em $\Omega_{1}$, mais ainda usando a hipótese $\left(H_{0}\right)^{\prime}$ obtemos que para um $B$ adequado

$$
\left\{\begin{aligned}
-\Delta\left(u_{0}-\underline{u}\right) & \geq f_{\lambda}\left(x, u_{0}\right)-f_{\lambda}(x, \underline{u}) \geq-B\left(u_{0}-\underline{u}\right), & & x \in \Omega_{1}, \\
\left(u_{0}-\underline{u}\right) & \geq 0, & & x \in \partial \Omega_{1},
\end{aligned}\right.
$$

assim pelo Princípio do Máximo Forte, temos

$$
\left\{\begin{aligned}
u_{0}-\underline{u}>0, & x \in \Omega_{1}, \\
\frac{\partial\left(u_{0}-\underline{u}\right)}{\partial \nu}<0, & x \in \partial \Omega_{1} .
\end{aligned}\right.
$$

Mostraremos agora que $u_{0}<\bar{u}$ em $\Omega_{1}$ e que $\frac{\partial u_{0}}{\partial \nu}>\frac{\partial \bar{u}}{\partial \nu}$ sobre $\partial \Omega_{1}$.

Como $\bar{u}$ é supersolução de $\left(\tilde{P}_{\lambda}\right)$, pela definição de $\Lambda$, existe $\bar{\lambda} \in \mathbb{R}$ tal que $\bar{u}$ é solução de $\left(\tilde{P}_{\lambda}\right)$ assim

$$
-\Delta \bar{u}=f_{\bar{\lambda}}(x, \bar{u})
$$

e como $\Omega_{1} \subset \Omega$ é um subdomínio, teríamos que

$$
\left\{\begin{aligned}
-\Delta \bar{u} & \geq f_{\lambda}(x, \bar{u}), & & x \in \Omega_{1}, \\
\bar{u} & \geq 0, & & x \in \partial \Omega_{1},
\end{aligned}\right.
$$


assim, utilizando a hipótese $(M)$, temos

$$
\left\{\begin{aligned}
-\Delta\left(\bar{u}-u_{0}\right) & =-\Delta \bar{u}-\left(-\Delta u_{0}\right)=f_{\bar{\lambda}}(x, \bar{u})-f_{\lambda}\left(x, u_{0}\right) \geq 0, & & x \in \Omega_{1}, \\
\left(\bar{u}-u_{0}\right) & \geq 0, & & x \in \partial \Omega_{1},
\end{aligned}\right.
$$

logo pelo Princípio do Máximo forte, temos que

$$
\left\{\begin{array}{cl}
\bar{u}-u_{0}>0, & x \in \Omega_{1} \\
\frac{\partial\left(\bar{u}-u_{0}\right)}{\partial \nu}<0, & x \in \partial \Omega_{1} .
\end{array}\right.
$$

De (2.16), (2.17) e (2.18) a afirmação segue.

Afirmação 2.4. $u_{0}$ é mínimo local de $I_{\lambda}$ sobre $H$.

De fato, de (2.15) temos que $\{u \in H: \underline{u} \leq u \leq \bar{u}\}$ contém uma vizinhança de $u_{0}$ em $C_{0}^{1}(\bar{\Omega})$. Mas por (2.6) temos que

$$
I_{\lambda}\left(u_{0}\right)=\min \left\{I_{\lambda}(u): u \in H, \underline{u} \leq u \leq \bar{u}\right\}
$$

logo $u_{0}$ é mínimo local de $I_{\lambda}$ sobre $C_{0}^{1}(\Omega)$. Mas como $\sigma<2^{*}-1$ (caso subcrítico) e pelo Teorema A.12, temos que $u_{0}$ é também um mínimo local de $I_{\lambda}$ sobre $H$. (Maiores detalhes ver Lema 1.7.)

A segunda solução será construída na forma $u_{0}+w$, onde $u_{0}$ é a primeira solução e $w$ satisfaz

$$
\left\{\begin{array}{rlrl}
-\Delta w & =g_{\lambda}(x, w), & & x \in \Omega, \\
w \neq 0, & & x \in \Omega, \\
w & =0, & & x \in \partial \Omega
\end{array}\right.
$$

onde $g_{\lambda}(x, s):=f_{\lambda}\left(x, u_{0}+s^{+}\right)-f_{\lambda}\left(x, u_{0}\right)$. Esta ferramenta, já foi considerada anteriormente com $a(x) \equiv$ $b(x) \equiv 1$.

Afirmação 2.5. A solução $w$ de (2.19) é não negativa.

Suponha por contradição que $w<0$. Agora multiplique (2.19) por $\left(-w^{-}\right)$. Assim temos que

$$
-\Delta w\left(-w^{-}\right)=-g_{\lambda}(x, w) w^{-},
$$

logo integrando sobre $\Omega$ e aplicando uma das identidades do Green, temos que

$$
\begin{aligned}
-\int_{\Omega} \nabla w\left(\nabla w^{-}\right) d x & =-\int_{\Omega} g_{\lambda}(x, w) w^{-} d x \\
\int_{\Omega}\left|\nabla w^{-}\right|^{2} d x & =-\int_{\Omega} g_{\lambda}(x, w) w^{-} d x \\
\left\|w^{-}\right\|^{2} & =-\int_{\Omega}\left[f_{\lambda}\left(x, u_{0}+w^{+}\right)-f_{\lambda}\left(x, u_{0}\right)\right] w^{-} d x .
\end{aligned}
$$

Como $f_{\lambda}$ satisfaz $\left(H_{0}\right)^{\prime}$, segue que

$$
\left[f_{\lambda}\left(x, u_{0}+w^{+}\right)-f_{\lambda}\left(x, u_{0}\right)\right] \geq 0,
$$


agora note que, como $w<0$ segue que $w^{-}=-w$ e assim $-w^{-}=w<0$. então temos que

$$
\left\|w^{-}\right\|^{2}=-\int_{\Omega}\left[f_{\lambda}\left(x, u_{0}+w^{+}\right)-f_{\lambda}\left(x, u_{0}\right)\right] w^{-} d x<0
$$

O que é uma contradição. Concluindo assim a afirmação.

Agora, consideramos

$$
\left\{\begin{aligned}
-\Delta w & =g_{\lambda}(x, w), & & x \in \Omega, \\
w & \geq 0, & & x \in \Omega, \\
w & =0, & & x \in \partial \Omega,
\end{aligned}\right.
$$

$\operatorname{mas} \operatorname{como} g_{\lambda}(x, s)=f_{\lambda}\left(x, u_{0}+s^{+}\right)-f_{\lambda}\left(x, u_{0}\right) \geq 0$ segue que

$$
\left\{\begin{aligned}
-\Delta w \geq 0, & x \in \Omega, \\
w=0, & x \in \partial \Omega .
\end{aligned}\right.
$$

Logo pelo Princípio do Máximo e a hipótese $\left(H_{0}\right)^{\prime}$ temos que $w$ satisfaz

$$
\left\{\begin{aligned}
-\Delta w>0, & x \in \Omega \\
\frac{\partial w}{\partial \nu}<0, & x \in \partial \Omega
\end{aligned}\right.
$$

onde $\nu$ é o vetor normal unitário exterior a $\partial \Omega$.

Assim, $u_{0}+w$ será a segunda solução de $\left(\tilde{P}_{\lambda}\right)$ a qual satisfaz as hipóteses do Teorema 2.4. Escrevendo

$$
G_{\lambda}(x, s):=\int_{0}^{s} g_{\lambda}(x, t) d t
$$

e

$$
J_{\lambda}(w):=\frac{1}{2} \int_{\Omega}|w|^{2}-\int_{\Omega} G_{\lambda}(x, w) d x .
$$

Assim, devemos procurar um ponto crítico não negativo de $J_{\lambda}$ sobre $H$. Concluindo assim a afirmação.

Afirmação 2.6. $J_{\lambda}(w)=I_{\lambda}\left(u_{0}+w^{+}\right)-I_{\lambda}\left(u_{0}\right)+\frac{1}{2}\left\|w^{-}\right\|^{2}$.

De fato,

$$
\begin{aligned}
G_{\lambda}\left(x, s^{+}\right)-F_{\lambda}\left(x, u_{0}+s^{+}\right) & =\int_{0}^{s^{+}} g_{\lambda}(x, t) d t-\int_{0}^{u_{0}+s^{+}} f_{\lambda}(x, t) d t \\
& =\int_{0}^{s^{+}}\left[f_{\lambda}\left(x, u_{0}+t\right)-f_{\lambda}\left(x, u_{0}\right)\right] d t-\int_{0}^{u_{0}+s^{+}} f_{\lambda}(x, t) d t \\
& =F_{\lambda}\left(x, u_{0}+s^{+}\right)-F_{\lambda}\left(x, u_{0}\right)-f_{\lambda}\left(x, u_{0}\right) s^{+}-F_{\lambda}\left(x, u_{0}+s^{+}\right)-F_{\lambda}(x, 0) \\
& =-F_{\lambda}\left(x, u_{0}\right)-f_{\lambda}\left(x, u_{0}\right) s^{+} .
\end{aligned}
$$

Então,

$$
\begin{aligned}
J_{\lambda}(w) & =\frac{1}{2}\left\|w^{+}\right\|^{2}+\frac{1}{2}\left\|w^{-}\right\|^{2}-\int_{\Omega} G_{\lambda}\left(x, w^{+}\right) d x \\
& =\frac{1}{2}\left\|w^{+}\right\|^{2}+\frac{1}{2}\left\|w^{-}\right\|^{2}-\int_{\Omega} F_{\lambda}\left(x, u_{0}+w^{+}\right) d x+\int_{\Omega} F_{\lambda}\left(x, u_{0}\right) d x+\int_{\Omega} f_{\lambda}\left(x, u_{0}\right) w^{+} d x .
\end{aligned}
$$


Por outro lado, temos que

$$
\begin{aligned}
I_{\lambda}\left(u_{0}+w^{+}\right) & =\frac{1}{2}\left\|u_{0}+w^{+}\right\|^{2}-\int_{\Omega} F_{\lambda}\left(x, u_{0}+w^{+}\right) d x \\
& =\frac{1}{2} \int_{\Omega}\left|\nabla\left(u_{0}+w^{+}\right)\right|^{2} d x-\int_{\Omega} F_{\lambda}\left(x, u_{0}+w^{+}\right) d x \\
& =\frac{1}{2}\left[\int_{\Omega}\left|\nabla u_{0}\right|^{2}+\left|\nabla w^{+}\right|^{2}+2 \nabla u_{0} \nabla w^{+} d x\right]-\int_{\Omega} F_{\lambda}\left(x, u_{0}+w^{+}\right) d x \\
& =\frac{1}{2} \int_{\Omega}\left|\nabla u_{0}\right|^{2} d x+\frac{1}{2} \int_{\Omega}\left|w^{+}\right|^{2} d x+\int_{\Omega} \nabla u_{0} \nabla w^{+} d x-\int_{\Omega} F_{\lambda}\left(x, u_{0}+w^{+}\right) d x \\
& =\frac{1}{2}\left\|u_{0}\right\|^{2}+\frac{1}{2}\left\|w^{+}\right\|^{2}+\left[\int_{\Omega} \frac{\partial u_{0}}{\partial \nu} w^{+} d S-\int_{\Omega} \Delta u_{0} w^{+} d x\right]-\int_{\Omega} F_{\lambda}\left(x, u_{0}+w^{+}\right) d x \\
& =\frac{1}{2}\left\|u_{0}\right\|^{2}+\frac{1}{2}\left\|w^{+}\right\|^{2}+\int_{\Omega}\left(-\Delta u_{0}\right) w^{+} d x-\int_{\Omega} F_{\lambda}\left(x, u_{0}+w^{+}\right) d x \\
& =\frac{1}{2}\left\|u_{0}\right\|^{2}+\frac{1}{2}\left\|w^{+}\right\|^{2}+\int_{\Omega} f_{\lambda}\left(x, u_{0}\right) w^{+} d x-\int_{\Omega} F_{\lambda}\left(x, u_{0}+w^{+}\right) d x .
\end{aligned}
$$

Agora, como

$$
J_{\lambda}(w)=\frac{1}{2}\left\|w^{+}\right\|^{2}+\frac{1}{2}\left\|w^{-}\right\|^{2}-\int_{\Omega} F_{\lambda}\left(x, u_{0}+w^{+}\right) d x+\int_{\Omega} F_{\lambda}\left(x, u_{0}\right) d x+\int_{\Omega} f_{\lambda}\left(x, u_{0}\right) w^{+} d x
$$

substituindo a expressão $-\int_{\Omega} F_{\lambda}\left(x, u_{0}+w^{+}\right) d x$ de $(2.21)$ em $(2.22)$, temos que

$$
\begin{aligned}
J_{\lambda}(w)= & \frac{1}{2}\left\|w^{+}\right\|^{2}+\frac{1}{2}\left\|w^{-}\right\|^{2}+\left[I_{\lambda}\left(u_{0}+w^{+}\right)-\frac{1}{2}\left\|u_{0}\right\|^{2}-\frac{1}{2}\left\|w^{+}\right\|^{2}-\int_{\Omega} f_{\lambda}\left(x, u_{0}\right) w^{+} d x\right] \\
& +\int_{\Omega} F_{\lambda}\left(x, u_{0}\right) d x+\int_{\Omega} f_{\lambda}\left(x, u_{0}\right) w^{+} d x \\
= & \frac{1}{2}\left\|w^{-}\right\|^{2}+I_{\lambda}\left(u_{0}+w^{+}\right)-\frac{1}{2}\left\|u_{0}\right\|^{2}+\int_{\Omega} F_{\lambda}\left(x, u_{0}\right) d x \\
= & \frac{1}{2}\left\|w^{-}\right\|^{2}+I_{\lambda}\left(u_{0}+w^{+}\right)-\left[\frac{1}{2}\left\|u_{0}\right\|^{2}-\int_{\Omega} F_{\lambda}\left(x, u_{0}\right) d x\right] \\
= & I_{\lambda}\left(u_{0}+w^{+}\right)-I_{\lambda}\left(u_{0}\right)+\frac{1}{2}\left\|w^{-}\right\|^{2} .
\end{aligned}
$$

Portanto,

$$
J_{\lambda}(w)=I_{\lambda}\left(u_{0}+w^{+}\right)-I_{\lambda}\left(u_{0}\right)+\frac{1}{2}\left\|w^{-}\right\|^{2} .
$$

Concluindo assim a afirmação.

Afirmação 2.7. $w=0$ é um minimo local de $J_{\lambda}$ sobre $H$.

De fato, é suficiente mostrar que $w=0$ é um mínimo local de $J_{\lambda}$ na topologia $C^{1}$. Por $(2.23)$ temos que

$$
J_{\lambda}(w)=I_{\lambda}\left(u_{0}+w^{+}\right)-I_{\lambda}\left(u_{0}\right)+\frac{1}{2}\left\|w^{-}\right\|^{2},
$$

$\operatorname{logo} J_{\lambda}(w) \geq 0$, pois pela afirmação (2.4) temos que $u_{0}$ é um mínimo local de $I_{\lambda}$, daí existe $r>0$ tal que

$$
I_{\lambda}\left(u_{0}+w^{+}\right)-I_{\lambda}\left(u_{0}\right) \geq 0, \text { para }\left\|w^{+}\right\|_{C^{1}} \leq r .
$$


Assim, $w=0$ é um mínimo local na topologia $C^{1}$. Logo $J_{\lambda}(0) \leq J_{\lambda}(w)$, para todo $w \in B(0, r)$ em $H$. Concluindo assim a afirmação.

Afirmação 2.8. Supondo a hipótese $(G)$ com $\sigma<2^{*}-1$ e $(A R)_{d}$ temos que $I_{\lambda}$ satisfaz a condição $(P S)$ sobre $H$.

De fato, primeiramente pela hipótese $\left(H_{0}\right), f$ é estendida sobre todo $\Omega \times \mathbb{R}$, escrevendo agora $f_{\lambda}(x, s)=$ $f_{\lambda}(x, 0)$ em quase todo ponto de $\Omega$ e todo $s<0$. Assim, é claro que $(G)$ implica que $I_{\lambda}$ é um funcional $C^{1}$ sobre $H$.

Seja $\left(u_{n}\right) \subset H$ uma sequência $(P S)$, isto é, $I_{\lambda}\left(u_{n}\right) \rightarrow c$ e $I_{\lambda}^{\prime}\left(u_{n}\right) \rightarrow 0$.

Assim, para $\theta$ como na condição $(A R)_{d}$, existe uma constante $C_{1}>0$ e uma sequência $\varepsilon_{n} \rightarrow 0$ tais que

$$
\theta I_{\lambda}\left(u_{n}\right)-I_{\lambda}^{\prime}\left(u_{n}\right) u_{n} \leq C_{1}+\varepsilon_{n}\left\|u_{n}\right\|
$$

Além disso, temos que

$$
\begin{aligned}
\theta I_{\lambda}\left(u_{n}\right)-I_{\lambda}^{\prime}\left(u_{n}\right) u_{n} & =\frac{\theta}{2}\left\|u_{n}\right\|^{2}-\theta \int_{\Omega} F_{\lambda}\left(x, u_{n}\right) d x-\left\|u_{n}\right\|^{2}+\int_{\Omega} f_{\lambda}\left(x, u_{n}\right) u_{n} d x \\
& =\left(\frac{\theta}{2}-1\right)\left\|u_{n}\right\|^{2}-\int_{\Omega}\left(\theta F_{\lambda}\left(x, u_{n}\right)-u_{n} f_{\lambda}\left(x, u_{n}\right)\right) d x \\
& \leq C_{1}+\varepsilon_{n}\left\|u_{n}\right\|
\end{aligned}
$$

onde a última integral pode escrever-se como

$$
\int_{\Omega}\left(\theta F_{\lambda}\left(x, u_{n}\right)-u_{n} f_{\lambda}\left(x, u_{n}\right)\right) d x=\int_{\Omega_{1_{n}} \cup \Omega_{2_{n}} \cup \Omega_{3_{n}}}\left(\theta F_{\lambda}\left(x, u_{n}\right)-u_{n} f_{\lambda}\left(x, u_{n}\right)\right) d x
$$

onde estamos denotando

$$
\begin{aligned}
& \Omega_{1_{n}}=\left\{x \in \Omega: u_{n}(x)<0\right\} \\
& \Omega_{2_{n}}=\left\{x \in \Omega: 0 \leq u_{n}(x) \leq s_{0}\right\} \\
& \Omega_{3_{n}}=\left\{x \in \Omega: u_{n}(x)>s_{0}\right\}
\end{aligned}
$$

Observe agora que,

$$
\int_{\Omega_{1_{n}}}\left(\theta F_{\lambda}\left(x, u_{n}\right)-u_{n} f_{\lambda}\left(x, u_{n}\right)\right) d x=0
$$

pois $f_{\lambda}(x, s)=F_{\lambda}(x, s)=0$, quando $s<0$.

Agora, por a hipótese $(G)$ existem $1 \leq \sigma<2^{*}-1, d_{1}, d_{2}>0$ tais que

$$
\left|f_{\lambda(x, s)}\right| \leq d_{1}+d_{2} s^{\sigma}
$$

em quase todo ponto de $\Omega$ e para todo $s \geq 0$. Integrando de 0 a $s$ temos que

$$
\left|F_{\lambda}(x, s)\right| \leq d_{1} s+d_{3}|s|^{\sigma+1}
$$


$\log \mathrm{O}$

$$
\begin{aligned}
\int_{\Omega_{2_{n}}}\left(\theta F_{\lambda}\left(x, u_{n}\right)-u_{n} f_{\lambda}\left(x, u_{n}\right)\right) d x & \leq \int_{\Omega_{2_{n}}}\left|F_{\lambda}\left(x, u_{n}\right)\right| d x+\int_{\Omega_{2_{n}}}\left|u_{n} f_{\lambda}\left(x, u_{n}\right)\right| d x \\
& \leq \theta \int_{\Omega_{2_{n}}}\left(u_{n} d_{1}+d_{3}\left|u_{n}\right|^{\sigma+1}\right) d x+\int_{\Omega_{2_{n}}}\left(\left.\left|u_{n} d_{1}+d_{2}\right| u_{n}\right|^{\sigma+1}\right) d x \\
& \leq \theta s_{0} d_{1} \int_{\Omega} d x+\theta s_{0}^{\sigma+1} d_{3} \int_{\Omega} d x+s_{0} d_{1} \int_{\Omega} d x+d_{3} s_{0}^{\sigma+1} \int_{\Omega} d x \\
& \leq C_{2}
\end{aligned}
$$

onde $C_{2}$ é uma constante positiva.

Considerando agora a hipótese $(A R)_{d}$, e a desigualdade de Hölder segue que

$$
\begin{aligned}
\int_{\Omega_{3_{n}}}\left(\theta F_{\lambda}\left(x, u_{n}\right)-u_{n} f_{\lambda}\left(x, u_{n}\right)\right) d x & \leq d \int_{\Omega_{3_{n}}} u_{n}^{\rho} d x \leq d \int_{\Omega}\left(u_{n}^{+}\right)^{\rho} d x \\
& \leq d\left(\int_{\Omega} 1^{\left(\frac{2^{*}}{\rho}\right)^{\prime}} d x\right)^{\frac{1}{\left(2^{*} / \rho\right)^{\prime}}}\left(\int_{\Omega}\left(u_{n}^{+}\right)^{2^{*}} d x\right)^{\left(\rho / 2^{*}\right)} \\
& =C_{3}\left\|u_{n}^{+}\right\|_{2^{*}}^{\rho} \leq C_{4}\left\|u_{n}^{+}\right\|^{\rho}
\end{aligned}
$$

Sendo que nesta última desigualdade, utilizamos a imersão de Sobolev $H \hookrightarrow L^{2^{*}}(\Omega)$. Logo, por $(2.24)$ e (2.25) temos que

$$
\left(\frac{\theta}{2}-1\right)\left\|u_{n}\right\|^{2} \leq C_{2}+C_{4}\left\|u_{n}^{+}\right\|^{\rho}+\varepsilon_{n}\left\|u_{n}\right\|
$$

como $\rho<2$, concluímos que $\left(u_{n}\right)$ é limitada em $H$.

Observemos agora que

$$
I^{\prime}(u)=u-L^{\prime}(u) \quad \text { em } H^{\prime},
$$

onde

$$
\begin{aligned}
L: H & \rightarrow \mathbb{R} \\
u & \mapsto L(u)=\int_{\Omega} F_{\lambda}(x, u) d x
\end{aligned}
$$

$$
L^{\prime}(u) v=\int_{\Omega} f_{\lambda}(x, u) v d x
$$

para $u, v \in H$. Mas pela Proposição A.2, temos que $L^{\prime}: H \rightarrow H^{\prime}$ é compacto. Logo, como $\left(u_{n}\right)$ é limitada, existe uma subsequência $\left(u_{n_{j}}\right) \subset\left(u_{n}\right)$ tal que

$$
L^{\prime}\left(u_{n_{j}}\right) \rightarrow v \text { em } H
$$

com isso,

$$
u_{n_{j}}=I^{\prime}\left(u_{n_{j}}\right)+L^{\prime}\left(u_{n_{j}}\right) \rightarrow v
$$

isto é, $\left(u_{n}\right)$ possui subsequência convergente. Assim, $I_{\lambda}$ satisfaz a condição $(P S)$, e concluímos a afirmação.

Agora, olharemos para uma relação em que 0 é um minimizador de $J_{\lambda}$. Assim, ou existe $w \in B(0, r)$ com $w \neq 0$ e $J_{\lambda}(w)=0$, ou $J_{\lambda}(0)<J_{\lambda}(w)$ para todo $w \in B(0, r)$ com $w \neq 0$.

No primeiro caso, $w$ é um mínimo local não-nulo de $J_{\lambda}$ e assim um ponto crítico de $J_{\lambda}$. Logo a prova 
está concluída.

No segundo caso, o Teorema 5.10 de [11] garante que para cada $r>0$ suficientemente pequeno

$$
J_{\lambda}(0)<\inf \left\{J_{\lambda}(w): w \in H \text { e }\|w\|=r\right\} .
$$

Nosso objetivo é aplicar o Teorema do Passo da Montanha.

Afirmação 2.9. Existe alguma função $u_{2} \in H$ tal que $J_{\lambda}\left(t u_{2}\right) \rightarrow-\infty$ quando $t \rightarrow \infty$.

De fato, pela hipótese $\left(H_{\Omega_{2}}\right)$ temos que, para todo $\lambda>0$, existe $\Omega_{2}, s_{2}$ e $\theta_{2}>0$ tal que

$$
F_{\lambda}(x, s) \geq \theta_{2} s^{2}
$$

em quase todo ponto de $\Omega_{2}$ e $s \geq s_{2}$.

E pela hipótese $(A R)_{d}$, existe $\theta>2, \rho<2, d \geq 0$ e $s_{0} \geq 0$ tal que

$$
\theta F_{\lambda}(x, s) \leq s f_{\lambda}(x, s)+d s^{\rho} .
$$

Tome $s_{3}=\max \left\{s_{0}, s_{2}\right\}$. Assim, temos que a hipótese $(A R)_{d}$ é

$$
\theta F_{\lambda}(x, s) \leq s f_{\lambda}(x, s)+d s^{\rho}
$$

em quase todo ponto de $\Omega_{2}$ e para todo $s \geq s_{3}$. Dividindo a expressão (2.27) por $s F_{\lambda}(x, s) \neq 0$ temos que

$$
\frac{\theta F_{\lambda}(x, s)}{s F_{\lambda}(x, s)} \leq \frac{s f_{\lambda}(x, s)}{s F_{\lambda}(x, s)}+\frac{d s^{\rho}}{s F_{\lambda}(x, s)}
$$

o que implica que

$$
\frac{\theta}{s} \leq \frac{f_{\lambda}(x, s)}{F_{\lambda}(x, s)}+\frac{d s^{\rho-1}}{F_{\lambda}(x, s)} .
$$

Integrando (2.28) de $s_{3}$ a $s$, utilizando a hipótese $\left(H_{\Omega_{2}}\right)$ e lembrando que $\rho<2$ temos

$$
\begin{aligned}
\int_{s_{3}}^{s} \frac{\theta}{t} d t & \leq \int_{s_{3}}^{s} \frac{f_{\lambda}(x, t)}{F_{\lambda}(x, t)} d t+d \int_{s_{3}}^{s} \frac{t^{\rho-1}}{F_{\lambda}(x, t)} d t \\
\ln \left(\frac{s}{s_{3}}\right)^{\theta} & \leq \ln \left(\frac{F_{\lambda}(x, s)}{F_{\lambda}\left(x, s_{3}\right)}\right)+d \int_{s_{3}}^{s} \frac{t^{\rho-1}}{\theta_{2} t^{2}} d t \\
& \leq \ln \left(\frac{F_{\lambda}(x, s)}{F_{\lambda}\left(x, s_{3}\right)}\right)+\frac{d}{\theta_{2}} \int_{s_{3}}^{\infty} t^{\rho-3} d t \\
& \leq \ln \left(\frac{F_{\lambda}(x, s)}{F_{\lambda}\left(x, s_{3}\right)}\right)-\frac{d s_{3}^{\rho-2}}{\theta_{2}(\rho-2)}
\end{aligned}
$$

$\log 0$

$$
-\frac{d s_{3}^{\rho-2}}{\theta_{2}(\rho-2)} \geq \ln \left[\left(\frac{s}{s_{3}}\right)^{\theta}\right]-\ln \left[\frac{F_{\lambda}(x, s)}{F_{\lambda}\left(x, s_{3}\right)}\right]=\ln \left[\frac{s^{\theta} F_{\lambda}\left(x, s_{3}\right)}{s_{3}^{\theta} F_{\lambda}(x, s)}\right] .
$$

Aplicando a função exponencial na expressão anterior, temos que

$$
F_{\lambda}(x, s) \geq F_{\lambda}\left(x, s_{3}\right)\left(\frac{s}{s_{3}}\right)^{\theta} \exp \left(\frac{d s_{3}^{\rho-2}}{\theta_{2}(\rho-2)}\right)=C_{1} s^{\theta}
$$


em quase todo ponto de $\Omega_{2}$, para todo $s \geq s_{3}, \theta>2$ pela hipótese $(A R)_{d}$ e onde $C_{1}$ é uma constante positiva.

Analogamente, obtemos para $G_{\lambda}$ que para algum $s_{3}^{\prime}$ e $C^{\prime}>0$,

$$
G_{\lambda}(x, s) \geq C^{\prime} s^{\theta}
$$

em quase todo ponto de $\Omega_{2}$, e todo $s \geq s_{3}^{\prime}$. Assim, tomamos uma função suave $u_{2}$ com suporte em $\Omega_{2}$ e $u_{2} \geq 0, u_{2} \not \equiv 0, F_{\lambda}(x, 0)=0$, e consideramos $t u_{2}$ onde $t$ é suficientemente grande. Logo

$$
\begin{aligned}
J_{\lambda}\left(t u_{2}\right) & =\frac{t^{2}}{2}\left\|u_{2}\right\|^{2}-\int_{\Omega} G_{\lambda}\left(x, t u_{2}\right) d x \\
& =\frac{t^{2}}{2}\left\|u_{2}\right\|^{2}-\int_{\Omega_{2}} G_{\lambda}\left(x, t u_{2}\right) d x \\
& \leq \frac{t^{2}}{2}\left\|u_{2}\right\|^{2}-\int_{\Omega_{2}} C^{\prime}\left(t u_{2}\right)^{\theta} d x \\
& \leq \frac{t^{2}}{2}\left\|u_{2}\right\|^{2}-C_{1} t^{\theta} \int_{\Omega_{2}}\left(u_{2}\right)^{\theta} d x \\
& =\frac{t^{2}}{2}\left\|u_{2}\right\|^{2}-C_{4} t^{\theta}
\end{aligned}
$$

onde $C_{4}$ é uma constante positiva. Como $\theta>2$, implica que na expressão anterior $J_{\lambda}\left(t u_{2}\right) \rightarrow-\infty$ quando $t \rightarrow \infty$. Concluindo assim a afirmação.

Da afirmação anterior, pode-se aplicar o Teorema do Passo da Montanha a $J_{\lambda}$, assim existe $w$ um ponto crítico de $J_{\lambda} \operatorname{com} J_{\lambda}(w)=c$ e $J_{\lambda}^{\prime}(w)=0$. Isto completa a prova do Teorema 2.4.

\subsection{Existência de uma segunda solução no caso crítico com $\sigma<1$}

Nesta seção mostraremos o Teorema 2.5.

Demonstração. Fixe $\lambda$ com $0<\lambda<\Lambda$. Procederemos como no começo da prova do Teorema (2.4), considerando $u_{0}$ o mínimo local de $I_{\lambda}$ sobre $H$ e só deve-se mostrar a existência de uma solução de (2.19), onde $g_{\lambda}(x, s)$ agora é da forma

$$
g_{\lambda}(x, s):=h_{\lambda}\left(x, u_{0}+s^{+}\right)-h_{\lambda}\left(x, u_{0}\right)+b(x)\left[\left(u_{0}+s^{+}\right)^{p}-u_{0}^{p}\right]
$$

lembrando que $f_{\lambda}(x, s)=h_{\lambda}(x, s)+b(x) s^{p}$. O funcional associado $J_{\lambda}$ fica

$$
J_{\lambda}(w)=\frac{1}{2}\|w\|^{2}-\int_{\Omega} G_{\lambda}(x, w) d x
$$

onde agora

$$
G_{\lambda}(x, s):=H_{\lambda}\left(x, u_{0}+s^{+}\right)-H_{\lambda}\left(x, u_{0}\right)-h_{\lambda}\left(x, u_{0}\right) s^{+}+b(x)\left[\frac{\left(u_{0}+s^{+}\right)^{p+1}-u_{0}^{p+1}}{p+1}-u_{0}^{p} s^{+}\right]
$$

onde

$$
H_{\lambda}(x, s):=\int_{0}^{s} h_{\lambda}(x, t) d t
$$


Como antes provamos que, 0 é um mínimo local de $J_{\lambda}$ em $H$. Assim, só deve-se mostrar a existência de um ponto crítico não trivial de $J_{\lambda}$.

Suponha por contradição que 0 é o único ponto crítico de $J_{\lambda}$. Logo, para alguma bola $B(0, r)$ em $H$,

$$
J_{\lambda}(0) \leq J_{\lambda}(w)
$$

para todo $w \in B(0, r)$. Utilizando o Lema $A .7$ e o Teorema 5.10 de [11] garante que para cada $r>0$ suficientemente pequeno, temos que

$$
0=J_{\lambda}(0)<\inf \left\{J_{\lambda}(w):, w \in H,\|w\|=r\right\}
$$

Nosso objetivo é aplicar o Teorema do Passo da Montanha. Para isso, mostraremos a existência de $u_{1} \neq 0, u_{1} \in H$ tal que

$$
J_{\lambda}\left(u_{1}\right)<0 \quad \text { e } \quad \inf _{\gamma \in \Gamma} \max _{t \in[0,1]} J_{\lambda}(\gamma(t))<c_{0}
$$

onde $c_{0}=\frac{S^{N / 2}}{N\|b\|_{\infty}^{(N-2) / 2}}$. Uma vez feito isso, asseguraremos a existência de um ponto crítico não trivial para $J_{\lambda}$, o que é uma contradição e a prova do Teorema (2.5) será concluída.

Para construir $u_{1}$, considere as funções da forma $t \psi_{\mu} \operatorname{com} t>0 \mathrm{e}$

$$
\psi_{\mu}(x):=d \xi(x)\left(\frac{\mu}{\mu^{2}+\left|x-x_{0}\right|}\right)^{(N-2) / 2}
$$

onde $\mu>0, x_{0}$ é da hipótese $(b), \xi$ é uma função suave não-negativa fixa, com $\xi \equiv 1$ perto de $x_{0}$ e com suporte em uma bola $B_{2}$ de centro $x_{0}$ (onde $B_{2}$ é escolhida tal que $\bar{B}_{2} \subset B_{1}$ e $b(x) \geq \varepsilon$ em quase todo ponto de $B_{2}$, para algum $\varepsilon>0$ ) e a constante normalizadora $d>0$ é tomada tal que $\psi_{1}$ satisfaz $-\Delta \psi_{1}=\psi_{1}^{(N+2) /(N-2)}$ perto de $x_{0}$.

Afirmação 2.10. Para cada $\mu>0, J_{\lambda}\left(t \psi_{\mu}\right) \rightarrow-\infty, t \rightarrow+\infty$.

Lembre-se que $p=2^{*}-1$, assim

$$
\begin{aligned}
J_{\lambda}\left(t \psi_{\mu}\right) & =\frac{t^{2}}{2}\left\|\psi_{\mu}\right\|^{2}-\int_{\Omega} G_{\lambda}\left(x, t \psi_{\mu}\right) d x \\
& =\frac{t^{2}}{2}\left\|\psi_{\mu}\right\|^{2}-\int_{\Omega} H_{\lambda}\left(x, t \psi_{m u}+u_{0}\right)-H_{\lambda}\left(x, u_{0}\right)+b(x)\left[\frac{\left(u_{0}+t \psi_{\mu}\right)^{p+1}-u_{0}^{p+1}}{p+1}-u_{0}^{p} t \psi_{\mu}\right] d x \\
& \leq \frac{t^{2}}{2}\left\|\psi_{\mu}\right\|^{2}-\int_{\Omega} H_{\lambda}\left(x, t \psi_{m u}+u_{0}\right)-H_{\lambda}\left(x, u_{0}\right)-\int_{\Omega} b(x)\left[\frac{\left(t \psi_{\mu}\right)^{p+1}}{p+1}+u_{0}^{p-1} \frac{\left(t \psi_{\mu}\right)^{2}}{2}\right] d x
\end{aligned}
$$


Agora, considerando que $h_{\lambda}$ satisfaz a hipótese $(G)$ e que $b \in L^{\infty}(\Omega)$, temos que

$$
\begin{aligned}
J_{\lambda}\left(t \psi_{\mu}\right) \leq & \frac{t^{2}}{2}\left\|\psi_{\mu}\right\|^{2}-\int_{\Omega}\left[\int_{0}^{t \psi_{\mu}+u_{0}} h_{\lambda}(x, s) d s-\int_{0}^{u_{0}} h_{\lambda}(x, s) d s\right] d x \\
& -\int_{\Omega} b(x) \frac{t^{p+1}}{p+1} \psi_{\mu}^{p+1} d x-\frac{t^{2}}{2} \int_{\Omega} b(x)\left(\psi_{\mu}\right)^{2} d x \\
= & \frac{t^{2}}{2}\left\|\psi_{\mu}\right\|^{2}-\int_{\Omega} \int_{u_{0}}^{t \psi_{\mu}+u_{0}} h_{\lambda}(x, s) d s d x \\
& -\|b\|_{\infty} \frac{t^{p+1}}{p+1} \int_{\Omega} \psi_{\mu}^{p+1} d x-\|b\|_{\infty} \frac{t^{2}}{2} \int_{\Omega} \psi_{\mu}^{2} d x \\
\leq & \frac{t^{2}}{2}\left\|\psi_{\mu}\right\|^{2}-\int_{\Omega} \int_{u_{0}}^{t \psi_{\mu}+u_{0}}\left(d_{1}+d_{2} s^{\sigma}\right) d s d x \\
& -\|b\|_{\infty} \frac{t^{p+1}}{p+1} \int_{\Omega} \psi_{\mu}^{p+1} d x-\|b\|_{\infty} \frac{t^{2}}{2} \int_{\Omega} \psi_{\mu}^{2} d x \\
\leq & \frac{t^{2}}{2}\left\|\psi_{\mu}\right\|^{2}-C-\|b\|_{\infty} \frac{t^{2^{*}}}{2^{*}} \int_{\Omega} \psi_{\mu}^{2^{*}} d x-\|b\|_{\infty} \frac{t^{2}}{2} \int_{\Omega} \psi_{\mu}^{2} d x,
\end{aligned}
$$

como $2^{*}>2$, para todo $N \geq 3$ segue que $J_{\lambda}\left(t \psi_{\mu}\right) \rightarrow-\infty$ quando $t \rightarrow+\infty$, assim existe $t=t_{\mu}>0$ tal que $J_{\lambda}\left(t_{\mu} \psi_{\mu}\right)<0$. Pelo Lema $(A .7)$ temos que para $\mu$ suficientemente pequeno,

$$
\inf _{\gamma \in \Gamma} \max _{t \in[0,1]} J_{\lambda}(\gamma(t))<c_{0}=\frac{S^{N / 2}}{N\|b\|_{\infty}^{(N-2) / 2}}
$$

onde $\left.\Gamma=\left\{\gamma \in C\left([0,1], H_{0}^{1}\right)\right), \gamma(0)=0, \gamma(1)=u_{1}=t_{\mu} \psi_{\mu}\right\}$, assim, $J_{\lambda}$ tem um ponto crítico não trivial, o que é uma contradição.

Portanto, o Problema $\left(\tilde{P}_{\lambda}\right)$ tem ao menos duas soluções no caso crítico com $\sigma<1$. Isto completa a prova do Teorema 2.5.

\subsection{Existência de uma segunda solução para o caso crítico com $\sigma<2^{*}-1$}

Nesta seção mostraremos o Teorema 2.6.

Demonstração. O argumento para mostrar que qualquer $\left(w_{n}\right)$ satisfazendo que $J_{\lambda}\left(w_{n}\right) \rightarrow c$ e $J_{\lambda}^{\prime}\left(w_{n}\right) \rightarrow$ 0 é limitada, é a seguinte.

Note que em nossa situação, $H_{\lambda}(x, s) \geq 0$ e assim $\theta$ da condição $(A R)_{d}$ para $h_{\lambda}$ pode ser escolhido tal que $2<\theta<p+1$. Logo estimaremos

$$
\Phi\left(w_{n}\right):=J_{\lambda}\left(w_{n}\right)-\frac{1}{\theta} J_{\lambda}^{\prime}\left(w_{n}\right)\left(u_{0}+w_{n}\right)
$$

Como $J_{\lambda}\left(w_{n}\right) \rightarrow c$, logo $\left|J_{\lambda}\left(w_{n}\right)\right| \leq c$ e como $J_{\lambda}^{\prime}\left(w_{n}\right) \rightarrow 0$, temos que $\left|J_{\lambda}^{\prime}\left(w_{n}\right) \varphi\right| \leq \varepsilon_{n}\|\varphi\|$, onde $\varepsilon_{n} \rightarrow 0$. Assim,

$$
\Phi\left(w_{n}\right) \leq c+\frac{\varepsilon_{n}}{\theta}\left\|u_{0}+w_{n}\right\|
$$


Por outro lado, estendendo $\Phi\left(w_{n}\right)$ obtemos

$$
\begin{aligned}
\Phi\left(w_{n}\right)= & J_{\lambda}\left(w_{n}\right)-\frac{1}{\theta} J_{\lambda}^{\prime}\left(w_{n}\right)\left(u_{0}+w_{n}\right) \\
= & \frac{1}{2}\left\|w_{n}\right\|^{2}-\int_{\Omega} G_{\lambda}\left(x, w_{n}\right) d x-\frac{1}{\theta}\left[\int_{\Omega}\left|\nabla w_{n} \| \nabla\left(u_{0}+w_{n}\right)\right| d x-\int_{\Omega} g_{\lambda}\left(x, w_{n}\right)\left(u_{0}+w_{n}\right) d x\right] \\
= & \frac{1}{2}\left\|w_{n}\right\|^{2}-\int_{\Omega} H_{\lambda}\left(x, u_{0}+w_{n}^{+}\right) d x-H_{\lambda}\left(x, u_{0}\right)-h_{\lambda}\left(x, u_{0}\right) w_{n}^{+} d x \\
& \left.+b(x) \frac{\left(u_{0}+w_{n}^{+}\right)^{p+1}-u_{0}^{p+1}}{p+1}-u_{0}^{p} w_{n}^{+}\right]-\frac{1}{\theta}\left[\int_{\Omega}\left|\nabla w_{n} \| \nabla u_{0}\right| d x+\int_{\Omega}\left|\nabla w_{n}\right|^{2} d x\right. \\
& \left.-\int_{\Omega}\left(h_{\lambda}\left(x, u_{0}+w_{n}^{+}\right)-h_{\lambda}\left(x, u_{0}\right)+b(x)\left[\left(u_{0}+w_{n}^{+}\right)^{p}-u_{0}^{p}\right]\right)\left(u_{0}+w_{n}^{+}\right) d x\right] \\
= & \left(\frac{1}{2}-\frac{1}{\theta}\right)\left\|w_{n}\right\|^{2}-\int_{\Omega}\left[H_{\lambda}\left(x, w_{n}^{+}+u_{0}\right)-\frac{1}{\theta} h_{\lambda}\left(x, u_{0}+w_{n}^{+}\right)\left(u_{0}+w_{n}^{+}\right) d x\right] \\
& -\left(\frac{1}{p+1}-\frac{1}{\theta}\right) \int_{\Omega} b(x)\left(u_{0}+w_{n}^{+}\right)^{p+1} d x+\int_{\Omega}\left[H_{\lambda}\left(x, u_{0}\right)+h_{\lambda}\left(x, u_{0}\right) w_{n}^{+}+b(x) \frac{u_{0}^{p+1}}{p+1}\right. \\
& \left.+\frac{1}{\theta} h_{\lambda}\left(x, u_{0}\right)\left(u_{0}+w_{n}^{+}\right)+b(x) \frac{u_{0}^{p+1}}{\theta}\right] d x .
\end{aligned}
$$

A última integral denotamos como $A_{n}:=A\left(w_{n}\right)$, assim temos

$$
\begin{aligned}
\Phi\left(w_{n}\right)= & \left(\frac{1}{2}-\frac{1}{\theta}\right)\left\|w_{n}\right\|^{2}-\int_{\Omega}\left[H_{\lambda}\left(x, w_{n}^{+}+u_{0}\right)-\frac{1}{\theta} h_{\lambda}\left(x, u_{0}+w_{n}^{+}\right)\left(u_{0}+w_{n}^{+}\right)\right] d x \\
& -\left(\frac{1}{p+1}-\frac{1}{\theta}\right) \int_{\Omega} b(x)\left(u_{0}+w_{n}^{+}\right)^{p+1} d x+A_{n} .
\end{aligned}
$$

Afirmação 2.11. An é um termo de primeira ordem, isto é $\left\|A_{n}\right\| \leq C_{1}+C_{2}\left\|w_{n}\right\|$ para algumas constantes positivas $C_{1}$ e $C_{2}$.

De fato, temos que

$$
A_{n}=\int_{\Omega}\left[H_{\lambda}\left(x, u_{0}\right)+h_{\lambda}\left(x, u_{0}\right) w_{n}^{+}+b(x) u_{0}^{p+1}\left(\frac{1}{p+1}+\frac{1}{\theta}\right)+\frac{1}{\theta} h_{\lambda}\left(x, u_{0}\right)\left(u_{0}+w_{n}^{+}\right)\right] d x .
$$

Como $h_{\lambda}$ satisfaz $(A R)_{d}$ temos que

$$
\theta H_{\lambda}\left(x, u_{0}\right) \leq u_{0} h_{\lambda}\left(x, u_{0}\right)+d u_{0}^{\rho},
$$

onde $\rho<2, \theta>2$. assim,

$$
H_{\lambda}\left(x, u_{0}\right) \leq \frac{u_{0}}{\theta} h_{\lambda}\left(x, u_{0}\right)+\frac{d}{\theta} u_{0}^{\rho},
$$

$\log 0$

$$
\begin{aligned}
\left|A_{n}\right| \leq \int_{\Omega}\left[\frac{\left|u_{0}\right|}{\theta}\left|h_{\lambda}\left(x, u_{0}\right)\right|+\frac{d}{\theta}\left|u_{0}\right|^{\rho}+\left|h_{\lambda}\left(x, u_{0}\right)\right|\left|w_{n}^{+}\right|\right. \\
\left.+b(x)\left|u_{0}\right|^{p+1}\left(\frac{1}{p+1}+\frac{1}{\theta}\right)+\frac{1}{\theta}\left|h_{\lambda}\left(x, u_{0}\right)\right|\left|u_{0}+w_{n}^{+}\right|\right] d x,
\end{aligned}
$$


como $h_{\lambda}(x, s)=f_{\lambda}(x, s)-b(x) s^{p}$, e pela hipótese $(H)$ segue que

$$
\left|h_{\lambda}(x, s)\right| \leq\left|f_{\lambda}(x, s)\right|+|b(x)||s|^{p} \leq A+\|b\|_{\infty}|s|^{p}
$$

e assim,

$$
\left|H_{\lambda}(x, s)\right|=\left|\int_{0}^{s} h_{\lambda}(x, t) d t\right| \leq \int_{0}^{s} A+\|b\|_{\infty} t^{p} d t=A s+\|b\|_{\infty} \frac{s^{p+1}}{p+1} .
$$

Logo temos que

$$
\begin{aligned}
\left|A_{n}\right| \leq \int_{\Omega}\left[\frac{\left|u_{0}\right|}{\theta} A\left|u_{0}\right|\right. & +\|b\|_{\infty} \frac{\left|u_{0}\right|^{p+1}}{p+1}+\frac{d}{\theta}\left|u_{0}\right|^{\rho}+1 \\
+ & \left(A\left|u_{0}\right|+\|b\|_{\infty} \frac{\left|u_{0}\right|^{p+1}}{p+1}\right)\left|w_{n}\right|+b(x)\left|u_{o}\right|^{p+1} C \\
& \left.+\frac{1}{\theta}\left(A\left|u_{0}\right|+\|b\|_{\infty} \frac{\left|u_{0}\right|^{p+1}}{p+1}\right)\left(\left|u_{0}\right|+\left|w_{n}\right|\right)\right] d x .
\end{aligned}
$$

Então $\left\|A_{n}\right\| \leq C_{1}+C_{2}\left\|w_{n}\right\|$, para alguma constante $C_{1}, C_{2}$, sendo

$$
\begin{gathered}
C_{1}=\int_{\Omega}\left[\frac{\left|u_{0}\right|^{2}}{\theta} A+\|b\|_{\infty} \frac{\left|u_{0}\right|^{p+1}}{p+1}+\frac{d}{\theta}\left|u_{0}\right|^{\rho}+1+b(x)\left|u_{0}\right|^{p+1} C\right. \\
\left.+\frac{1}{\theta}\left(A\left|u_{0}\right|+\|b\|_{\infty} \frac{\left|u_{0}\right|^{p+1}}{p+1}\right)\left(\left|u_{0}\right|\right)\right] d x \\
C_{2}=\int_{\Omega}\left[A\left|u_{0}\right|+\|b\|_{\infty} \frac{\left|u_{0}\right|^{p+1}}{p+1}+\frac{1}{\theta}\left(A\left|u_{0}\right|+\|b\|_{\infty} \frac{\left|u_{0}\right|^{p+1}}{p+1}\right)\right] d x .
\end{gathered}
$$

Concluindo assim a afirmação.

Portanto de (2.31) e (2.32) temos que

$$
\begin{gathered}
\left(\frac{1}{2}-\frac{1}{\theta}\right)\left\|w_{n}\right\|^{2}=\int_{\Omega}\left[H_{\lambda}\left(x, u_{0}+w_{n}^{+}\right)-\frac{1}{\theta} h_{\lambda}\left(x, u_{0}+w_{n}^{+}\right)\left(u_{0}+w_{n}^{+}\right)\right] d x \\
+\left(\frac{1}{p+1}-\frac{1}{\theta}\right) \int_{\Omega} b(x)\left(u_{0}+w_{n}^{+}\right)^{p+1} d x+A_{n}^{\prime}
\end{gathered}
$$

para um outro termo de primeira ordem $A_{n}^{\prime}$.

Considerando a hipótese $(A R)_{d}, 2<\theta<p+1$ e $b(x) \geq 0$, temos que $w_{n}$ é limitada. Logo, $J_{\lambda}$ satisfaz $(P S)$. Isto completa a prova do Teorema (2.6).

\subsection{Aplicações}

Como aplicação dos resultados anteriores, consideraremos agora o seguinte problema:

$$
\left\{\begin{aligned}
-\Delta u & =\lambda a(x) u^{q}+b(x) u^{p}, & & x \in \Omega, \\
u & >0, & & x \in \Omega, \\
u & =0, & & x \in \partial \Omega,
\end{aligned}\right.
$$

onde $0 \leq q<1<p$, e agora consideramos

$$
f_{\lambda}(x, s)=\lambda a(x) s^{q}+b(x) s^{p}
$$


sendo assim que $I_{\lambda}$ é da seguinte forma

$$
I_{\lambda}(u)=\frac{1}{2} \int_{\Omega}|\nabla u|^{2} d x-\frac{\lambda}{q+1} \int_{\Omega} a(x)\left(u^{+}\right)^{q+1} d x-\frac{1}{p+1} \int_{\Omega} b(x)\left(u^{+}\right)^{p+1} d x .
$$

Teorema 2.7. Seja $0 \leq q<1<p$ e suponha que $a, b \in L^{\infty}(\Omega)$ com

(i) $a(x) \geq 0$ em quase todo ponto de $\Omega$,

(ii) $a(x) \geq \varepsilon_{1}>0$ em quase todo ponto de alguma bola $B_{1}$.

Então existe $0<\Lambda<\infty$ tal que o problema (2.33) tem ao menos uma solução u com $I_{\lambda}(u)<0$ para $0<\lambda<\Lambda$ e não tem solução para $\lambda>\Lambda$. Se além disso,

(iii) $b(x) \geq 0$ em quase todo ponto de alguma bola $B_{2}$ com $a(x), b(x) \not \equiv 0$ sobre $B_{2}$.

Então $\Lambda<\infty$. Mais ainda, se $p \leq 2^{*}-1$ logo o problema (2.33) tem ao menos uma solução u com $I_{\lambda}(u) \leq 0$ para $\lambda=\Lambda$.

Demonstração. É suficiente verificar as hipóteses dos Teoremas $(2.1) ;(2.2)$ e (2.3).

- Verificando a hipótese $(H)$.

De fato, como $a, b \in L^{\infty}(\Omega)$ segue que para qualquer $s_{0}>0$

$$
\begin{aligned}
\left|f_{\lambda}(x, s)\right| & =\left|\lambda a(x) s^{q}+b(x) s^{p}\right| \leq\|a\|_{\infty} \lambda s^{q}+\|b\|_{\infty} s^{p} \\
& \leq\|a\|_{\infty} \lambda s_{0}^{q}+\|b\|_{\infty} s_{0}^{p}=A,
\end{aligned}
$$

em quase todo ponto de $\Omega$ e todo $s \in\left[0, s_{0}\right]$. Assim, existe uma constante $A$ tal que satisfaz a hipótese $(H)$.

- Verificando a hipótese $\left(H_{0}\right)$.

Para cada $\lambda>0$ e cada $s_{0}>0$, temos que

$$
f_{\lambda}(x, s)=\lambda a(x) s^{q}+b(x) s^{p} \geq b(x) s^{p} \geq-b(x) s .
$$

Por outro lado, existe $x \in \Omega$ fixado tal que $b(x)=\|b\|_{\infty}$, assim tomando $B=\|b\|_{\infty}>0$, temos que a hipótese $\left(H_{0}\right)$ é satisfeita.

- Verificando a hipótese $\left(H_{e}\right)$.

Tome $g(s)=\lambda\|a\|_{\infty} s^{q}+\|b\|_{\infty} s^{p}$ com $\lambda$ suficientemente pequeno, assim

Afirmação: $g$ é não decrescente.

De fato, seja $s_{1}<s_{2}, \operatorname{logo}$

$$
\begin{aligned}
g\left(s_{1}\right) & =\lambda\|a\|_{\infty} s_{1}^{q}+\|b\|_{\infty} s_{1}^{p} \\
& <\lambda\|a\|_{\infty} s_{2}^{q}+\|b\|_{\infty} s_{2}^{p}=g\left(s_{2}\right) .
\end{aligned}
$$

Concluindo a afirmação.

Afirmação: $f_{\lambda}(x, s) \leq g(s)$.

De fato,

$$
\begin{aligned}
f_{\lambda}(x, s) & =\lambda a(x) s^{q}+b(x) s^{p} \\
& \leq \lambda\|a\|_{\infty} s^{q}+\|b\|_{\infty} s^{p}=g(s),
\end{aligned}
$$


em quase todo ponto de $\Omega$ e todo $s \geq 0$. Concluindo afirmação.

Portanto, das afirmações anteriores temos que a hipótese $\left(H_{e}\right)$ é satisfeita.

- Verificando a hipótese $\left(H_{\Omega_{1}}\right)$.

De fato, para toda $\lambda>0$, por (ii) temos que

$$
f_{\lambda}(x, s)=\lambda a(x) s^{q}+b(x) s^{p} \geq \lambda \varepsilon_{1} s^{q}+b(x) s^{p},
$$

em quase todo ponto de alguma bola $B_{1}$, assim tomando $\Omega_{1}=B_{1}$ temos que

$$
f_{\lambda}(x, s) \geq \lambda \varepsilon_{1} s^{q}+b(x) s^{p} \geq \lambda \varepsilon s^{q} \geq \lambda \varepsilon_{1} s .
$$

Tomando $\lambda \varepsilon=\theta_{1}>\lambda_{1}\left(\Omega_{1}\right)$, a hipótese é verificada.

Assim, do resultado do Teorema (2.1) segue que existe $0<\Lambda<\infty$ tal que o problema (2.33) tem ao menos uma solução $u$ com $I_{\lambda}(u)<0$ para $0<\lambda<\Lambda$ e não tem solução para $\lambda>\Lambda$.

Agora para a segunda parte do resultado, precisamos verificar as seguintes hipóteses:

- Verificando a hipótese $\left(H_{\tilde{\Omega}}\right)$.

Da hipótese (iii) temos que $b(x) \geq 0$ em quase todo ponto de alguma bola $B_{2}$, com $a(x), b(x) \not \equiv 0$ sobre $B_{2}$.

Temos que $f_{\lambda}(x, s)=\lambda a(x) s^{q}+b(x) s^{p}$.

Afirmação 2.12. Seja $A, B \geq 0 e 0 \leq q<1<p$ logo existe $c=c(p, q)>0$ tal que

$$
A s^{q}+B s^{p} \geq c A^{\frac{p-1}{p-q}} B^{\frac{1-q}{p-q}} S
$$

para todo $s \geq 0$.

De fato, seja $s>1$ e pela desigualdade de Young temos que

$$
\begin{aligned}
A^{\frac{p-1}{p-q}} B^{\frac{1-q}{p-q}} s & =(A s)^{\frac{p-1}{p-q}}(B s)^{\frac{1-q}{p-q}} \\
& \leq\left(\frac{p-1}{p-q}\right) A s+\left(\frac{1-q}{p-q}\right) B s \\
& \leq\left(\frac{p-1}{p-q}\right) A s^{q}+\left(\frac{1-q}{p-q}\right) B s^{p} \\
& \leq \max \left\{\frac{p-1}{p-q}, \frac{1-q}{p-q}\right\}\left(A s^{q}+B s^{p}\right) .
\end{aligned}
$$

Tomando $c(p, q)=\frac{1}{\max \left\{\frac{p-1}{p-q}, \frac{1-q}{p-q}\right\}}$ a afirmação segue. De modo análogo mostra-se para $s=0 \mathrm{e}$ $0<s<1$.

Assim, tomando $\tilde{\Omega}=B_{2}$ (subdomínio suave), $\tilde{m}=a(x)^{\frac{p-1}{p-q}} b(x)^{\frac{1-q}{p-q}}$ e $h(\lambda)=\lambda \frac{1}{\max \left\{\frac{p-1}{p-q}, \frac{1-q}{p-q}\right\}}$. Temos pela afirmação que

$$
f_{\lambda}(x, s)=\lambda a(x) s^{q}+b(x) s^{p} \geq h(\lambda) \tilde{m}(x) s,
$$

verificando-se a hipótese $\left(H_{\tilde{\Omega}}\right)$. Logo, o resultado do Teorema 2.2 conclui a segunda parte do Teorema 2.7 . 
Para finalizar a prova do Teorema 2.7 só falta mostrar as hipótese $(G)$ e $(A R)_{d}$.

- Verificando a hipótese $(G)$.

Para todo $[r, R] \subset\{\lambda>0\}$

$$
\begin{aligned}
\left|f_{\lambda}(x, s)\right| & =\left|\lambda a(x) s^{q}+b(x) s^{p}\right| \\
& \leq \lambda|a(x)| s^{q}+|b(x)| s^{p} \\
& \leq \lambda\|a\|_{\infty} s^{q}+\|b\|_{\infty} s^{p} \\
& \leq \lambda\|a\|_{\infty} s^{p}+\|b\|_{\infty} s^{p} \\
& =\left(\lambda\|a\|_{\infty}+\|b\|_{\infty}\right) s^{p}
\end{aligned}
$$

em quase todo ponto de $\Omega$ para todo $s \geq 0$. Assim, tomando $d_{1}=0, d_{2}=\lambda\|a\|_{\infty}+\|b\|_{\infty} \mathrm{e}$ $p=\sigma$; verifica-se a hipótese $(G)$.

- Verificando a hipótese $(A R)_{d}$.

Tome $\theta=p+1, \rho=q+1, d=R\left(\frac{\theta}{p+1}-1\right)\|a\|_{\infty}$ e $s_{0}=0$, temos que

$$
\begin{aligned}
\theta F_{\lambda}(x, s)-s f_{\lambda}(x, s) & =\theta \int_{0}^{s} f_{\lambda}(x, t) d t-s\left(\lambda a(x) s^{q}+b(x) s^{p}\right) \\
& =\theta \int_{0}^{s} \lambda a(x) t^{q}+b(x) t^{p} d t-\lambda a(x) s^{q+1}-b(x) s^{p+1} \\
& =(p+1)\left[\frac{\lambda}{q+1} a(x) s^{q+1}+\frac{b(x)}{p+1} s^{p+1}\right]-\lambda a(x) s^{q+1}+b(x) s^{p+1} \\
& =\left[\left(\frac{p+1}{q+1}\right)-1\right] \lambda a(x) s^{q+1} \leq\left[\left[\left(\frac{p+1}{q+1}\right)-1\right] R\|a\|_{\infty} s^{q+1}\right.
\end{aligned}
$$

para todo $\lambda \in[r, R], s \geq 0$. Assim, a hipótese $(A R)_{d}$ é satisfeita.

Logo, a última parte do Teorema 2.7 segue do resultado do Teorema 2.3. Isto completa a prova do Teorema 2.7.

Teorema 2.8. Seja $0 \leq q<1<p$ e suponha $a, b \in L^{\infty}(\Omega)$ com

(i) $a(x) \geq 0$ em quase todo ponto de $\Omega$,

(ii) $a(x) \geq \varepsilon_{1}>0$ em quase todo ponto de alguma bola $B_{1}$.

Suponha ainda que, ou $p<2^{*}-1 e$

(iv) $b(x) \geq \varepsilon_{2}>0$ em quase todo ponto de alguma bola $B_{2}$,

ou $p=2^{*}-1$ e a condiçãa (b) do Teorema 2.5 para $b(x)$.

Então o problema tem ao menos duas soluçôes $u, v$ para $0<\lambda<\Lambda$, com $u<v$ em $\Omega, \frac{\partial u}{\partial \nu}>\frac{\partial v}{\partial \nu}$ sobre $\partial \Omega$ e $I_{\lambda}(u)<0$.

Note que $(b)$ é uma condição mais forte que $(i v)$. Note também que $b(x)$ acima é permitido mudar de sinal em $\Omega$.

Demonstração. É suficiente verificar as hipóteses dos Teoremas 2.4 e 2.5. A verificação das hipóteses $(H),\left(H_{0}\right),\left(H_{e}\right),\left(H_{\Omega_{1}}\right),(G)$ e $(A R)_{d}$ é como no Teorema anterior. Apenas falta verificar $\left(H_{0}\right)^{\prime},(M) \mathrm{e}$ $\left(H_{\Omega_{2}}\right)$. 
- Verificação da Hipótese $\left(H_{0}\right)^{\prime}$

Para todo $\lambda>0$, e para todo $s_{0}>0$, seja $s_{1}, s_{2} \in\left[0, s_{0}\right]$ tal que $s_{1}<s_{2}$, tomando $B=\|a\|_{\infty}+\|$ $b \|_{\infty}$ segue que

$$
\begin{aligned}
f_{\lambda}\left(x, s_{1}\right)+B s & =\lambda a(x) s_{1}^{q}+b(x) s_{1}^{p}+\left(\|a\|_{\infty}+\|b\|_{\infty}\right) s \\
& \leq \lambda a(x) s_{2}^{q}+b(x) s_{2}^{p}+\left(\|a\|_{\infty}+\|b\|_{\infty}\right) s \\
& =f_{\lambda}\left(x, s_{2}\right)+B s .
\end{aligned}
$$

Verificando-se a hipótese $\left(H_{0}\right)^{\prime}$.

- Verificação da Hipótese $(M)$

Para todo $\lambda<\lambda^{\prime}$ e para todo $u \in C_{0}^{1}(\bar{\Omega}) \operatorname{com} u>0$ em $\Omega$,

$$
f_{\lambda}(x, u(x))=\lambda a(x) u^{q}+b(x) u^{p} \leq \lambda^{\prime} a(x) u^{q}+b(x) u^{p}=f_{\lambda^{\prime}}(x, u(x)) .
$$

Verificando-se a hipótese $(M)$.

- Verificação da Hipótese $\left(H_{\Omega_{2}}\right)$ Para todo $\lambda>0$,

$$
\begin{aligned}
F_{\lambda}(x, s) & =\int_{0}^{s} f_{\lambda}(x, t) d t=\int_{0}^{s} \lambda a(x) t^{q}+b(x) t^{p} d t \\
& =\frac{\lambda}{q+1} a(x) s^{q+1}+\frac{b(x)}{p+1} s^{p+1} \\
& \geq \frac{\lambda}{q+1} a(x) s^{q+1}+\varepsilon_{2} \frac{1}{p+1} s^{p+1} \\
& \geq \varepsilon_{2} \frac{1}{p+1} s^{p+1} \geq \varepsilon_{2} \frac{1}{p+1} s^{2} .
\end{aligned}
$$

Tomando $\theta_{2}=\frac{\varepsilon_{2}}{p+1}, \Omega_{2}=B_{2}$ e $s_{2}=1$, verifica-se a hipótese $H_{\Omega_{2}}$.

Assim, aplicando o Teorema 2.4 segue que o problema $\left(P_{\lambda}\right)$ com $p<2^{*}-1$ tem ao menos uma duas soluções $u, v$ para $0<\lambda<\Lambda$, com $u<v$ em $\Omega, \frac{\partial u}{\partial \nu}>\frac{\partial v}{\partial \nu}$ sobre $\partial \Omega$ e $I_{\lambda}(u)<0$.

Agora, no caso $p=2^{*}-1 \mathrm{com}$ as hipóteses o Teorema 2.5 satisfeitas. Isto completa a prova do Teorema 2.8 .

Agora resolveremos o problema,

$$
\left(P_{\lambda}\right)_{*}\left\{\begin{aligned}
-\Delta u & =\lambda c(x)(u+1)^{p}, & & x \in \Omega, \\
u & >0, & & x \in \Omega, \\
u & =0, & & x \in \partial \Omega,
\end{aligned}\right.
$$

onde $1<p \leq 2^{*}-1, c(x) \geq 0$.

Aqui o funcional associado fica

$$
I_{\lambda}(u)=\frac{1}{2} \int_{\Omega}|\nabla u|^{2} d x-\frac{\lambda}{p+1} \int_{\Omega} c(x)\left(u^{+}+1\right)^{p+1} d x .
$$

Teorema 2.9. Seja $p>1$ e suponha $c \in L^{\infty}(\Omega)$ com

$c(x) \geq 0$ em quase todo ponto de $\Omega$ e $c(x) \geq \varepsilon>0$ em quase todo ponto de alguma bola $B$. 
Então existe $0<\Lambda<\infty$ tal que $\left(P_{\lambda}\right)_{*}$ tem ao menos uma solução $u$, com $I_{\lambda}(u)<0$ para $0<\lambda<\Lambda$ e não tem solução para $\lambda>\Lambda$. Mais ainda, se $p \leq 2^{*}-1$, logo o problema $\left(P_{\lambda}\right)_{*}$ tem ao menos uma solução $u, \operatorname{com} I_{\lambda}(u) \leq 0$ para $\lambda=\Lambda$.

Demonstração. Deve-se verificar as hipóteses dos Teoremas 2.1, 2.2 e 2.3.

\section{- Verificação da Hipótese $(H)$}

De fato, para cada $\lambda>0$, temos que

$$
\left|f_{\lambda}(x, s)\right|=\left|\lambda c(x)(s+1)^{p}\right| \leq \lambda\|c\|_{\infty}\left|s_{0}+1\right|^{p}
$$

em quase todo ponto de $\Omega$ e para todo $s \in\left[0, s_{0}\right]$. Assim, tomando $A=\lambda\|c\|_{\infty}\left|s_{0}+1\right|^{p}$, a hipótese $(H)$ é verificada.

- Verificação da Hipótese $\left(H_{0}\right)$

De fato, para cada $\lambda>0$ e cada $s_{0}>0$, temos que

$$
\begin{aligned}
f_{\lambda}(x, s) & =\lambda c(x)(s+1)^{p} \\
& \geq \lambda c(x)\left(s^{p}+1\right) \\
& =\lambda c(x) s^{p}+\lambda c(x) \\
& \geq \lambda c(x) s^{p} \\
& \geq-\lambda c(x) s .
\end{aligned}
$$

Por outro lado, existe $x \in \Omega$ tal que $c(x)=\|c\|_{\infty}$. Asim, tomando $B=\lambda\|c\|_{\infty}$, temos que a hipótese $\left(H_{0}\right)$ é satisfeita.

- Verificação da Hipótese $\left(H_{e}\right)$

Tome $g(s)=\lambda\|c\|_{\infty}(s+1)^{p}$ para $\lambda$ suficientemente pequeno, logo

$$
f_{\lambda}(x, s)=\lambda c(x)(s+1)^{p} \leq \lambda\|c\|(s+1)^{p}=g(s),
$$

em quase todo ponto de $\Omega$ e todo $s \geq 0$. Verificando-se assim a hipótese $\left(H_{e}\right)$.

- Verificação da Hipótese $\left(H_{\Omega_{1}}\right)$

Para todo $\lambda>0$, temos que

$$
f_{\lambda}(x, s)=\lambda c(x)(s+1)^{p} \geq \lambda \varepsilon\left(s^{p}+1\right) \geq \lambda \varepsilon s^{p} \geq \lambda \varepsilon s,
$$

em quase todo ponto de $B_{1}$ e todo $s \in\left[0, s_{1}\right]$. Tomando $\Omega_{1}=B, \theta_{1}=\lambda \varepsilon>\lambda_{1}\left(\Omega_{1}\right)$ a hipótese $\left(H_{\Omega_{1}}\right)$ é verificada.

\section{- Verificação da Hipótese $\left(H_{\tilde{\Omega}}\right)$}

Como por hipótese $c(x) \geq \varepsilon>0$ em alguma bola $B$ segue que

$$
f_{\lambda}(x, s)=\lambda c(x)(s+1)^{p} \geq \lambda c(x) s^{p}+\lambda c(x) .
$$

Tomando $\tilde{\Omega}=B, h(\lambda)=\lambda$ e $\tilde{m}(x)=\varepsilon$ verifica-se a hipótese $\left(H_{\tilde{\Omega}}\right)$. 
- Verificação da Hipótese $(G)$

Para todo $[r, R] \subset\{\lambda>0\}$, temos que

$$
\begin{aligned}
\left|f_{\lambda}(x, s)\right| & =\left|\lambda c(x)(s+1)^{p}\right| \leq \lambda\|c\|_{\infty}|s+1|^{p} \\
& \leq \lambda\|c\|_{\infty} M\left(s^{p}+1\right) \\
& =\lambda\|c\|_{\infty} M s^{p}+\lambda\|c\|_{\infty} M .
\end{aligned}
$$

Tomando $d_{1}=\lambda\|c\|_{\infty} M, d_{2}=\lambda\|c\|_{\infty} M$ e $\sigma=p$ segue a verificação de $(G)$.

- Verificação da Hipótese $(A R)_{d}$

Para todo $[r, R] \subset\{\lambda>0\}$, temos que

$$
\begin{aligned}
\theta F_{\lambda}(x, s)-s f_{\lambda}(x, s) & =\theta \int_{0}^{s} f_{\lambda}(x, t) d t-s\left(\lambda c(x)(s+1)^{p}\right) \\
& =\theta \int_{0}^{s} \lambda c(x)(t+1)^{p} d t-s \lambda c(x)(s+1)^{p} \\
& =\theta \lambda c(x) \frac{(s+1)^{p+1}}{p+1}-s \lambda c(x)(s+1)^{p} \\
& =\lambda c(x)(s+1)^{p}\left[\left(\frac{\theta}{p+1}-1\right)(s+1)+1\right] \\
& \leq R\|c\|_{\infty}\left[\left(\frac{\theta}{p+1}-1\right)(s+1)+1\right](s+1)^{p} .
\end{aligned}
$$

Assim, escolhendo $\theta$ com $2<\theta<p+1$, o lado direito da desigualdade acima é $\leq 0$ para $s$ suficientemente grande, $\operatorname{logo}(A R)_{d}$ é satisfeito com $d=0$. Isto completa a prova do Teorema 2.9. 


\section{Apêndice}

\section{Resultados Importantes}

Teorema A.1. (Teorema da Divergência)(ver [16]). Seja $\Omega \subset \mathbb{R}^{N}$ um aberto limitado com fronteira regular, $F: \Omega \rightarrow \mathbb{R}^{N}$ é um campo vetorial em $\Omega, F \in C^{1}(\bar{\Omega})$, $\nu$ é o vetor normal unitário exterior a $\partial \Omega$. Então,

$$
\int_{\Omega} \nabla \cdot F(x) d x=\int_{\partial \Omega} F \cdot \nu d S .
$$

Teorema A.2. (As Identidades de Green)(ver [16]). Seja $\Omega \subset \mathbb{R}^{N}$ um dominio onde vale o teorema da divergência e sejam $u, v \in C^{2}(\bar{\Omega})$. Então valem as seguintes identidades:

1. $\int_{\Omega} \Delta u d x=\int_{\partial \Omega} \frac{\partial u}{\partial \nu} d S ;$

2. $\int_{\Omega} \nabla v \cdot \nabla u d x=-\int_{\Omega} u \Delta v d x+\int_{\partial \Omega} \frac{\partial v}{\partial \nu} u d S$

3. $\int_{\Omega} u \Delta v-v \Delta u d x=\int_{\partial \Omega} u \frac{\partial v}{\partial \nu}-v \frac{\partial u}{\partial \nu} d S$, onde $\frac{\partial}{\partial \nu}$ é a derivada direcional na direção do vetor unitário exterior $\nu$.

Definição A.1. (Espaço Paracompacto)(ver [15]) Um espaço topológico $X$ diz-se paracompacto se é Hausdorff e cada cobrimento aberto de $X$ tem um refinamento aberto localmente finito.

Teorema A.3. (Desigualdade do Valor Médio)(ver [14]). Dado $\Omega \subset \mathbb{R}^{N}$ um aberto, seja $f: \Omega \rightarrow \mathbb{R}^{m}$ diferenciável em cada ponto do segmento de reta aberto $(a, a+v)$ e tal que sua restrição ao segmento fechado $[a, a+v] \subset \Omega$ seja continua. Se $\left|f^{\prime}(x)\right| \leq M$ para todo $x \in(a, a+v)$, então

$$
|f(a+v)-f(a)| \leq M|v|
$$

Consideramos agora o problema

$$
\left\{\begin{aligned}
L u=f, & x \in \Omega, \\
u=0, & x \in \partial \Omega
\end{aligned}\right.
$$


onde $\Omega$ é um aberto e limitado contido em $\mathbb{R}^{N}$ e $u: \bar{\Omega} \rightarrow \mathbb{R}$ é desconhecida, $u=u(x)$. Onde $f: \Omega \rightarrow \mathbb{R}$ é conhecida, e $L$ denota os operadores elípticos de segunda ordem, que são da seguinte forma:

$$
L u:=-\sum_{i, j=1}^{N} a^{i j}(x) u_{x_{i} x_{j}}+\sum_{i=1}^{N} b^{i}(x) u_{x_{i}}+c(x) u,
$$

onde $a^{i j}, b^{i}, c: \Omega \rightarrow \mathbb{R}$, são funções contínuas. Sobre esse formato, apresentamos o seguinte resultado.

Lema A.1. (Lema de Hopf)(ver [18]). Seja $\Omega \in \mathbb{R}^{N}$, suponha $u \in C^{2} \cap(\Omega) C^{1}(\bar{\Omega})$ e $c \equiv 0$ em $\Omega$. Suponha ainda que Lu $\geq 0$ em $\Omega$ e que exista um ponto $x_{0} \in \partial \Omega$ tal que $u\left(x_{0}\right)>u(x)$ para todo $x \in \Omega$. Finalmente, suponha que existe uma bola $B \subset \Omega$ com $x_{0} \in \partial \Omega$. Logo,

1. $\frac{\partial u}{\partial \nu}\left(x_{0}\right)<0$, onde $\nu$ é o vetor normal unitário exterior a $B$ em $x_{0}$;

2. Se c $\geq 0$ em $\Omega$, a mesma conclusão do item anterior é valida sempre que $u\left(x_{0}\right) \geq 0$.

Teorema A.4. (Princípio do Máximo)(ver [18]). Seja $u \in H$ solução de

$$
\left\{\begin{aligned}
-\Delta u+\lambda u=h, & x \in \Omega, \\
u=0, & x \in \partial \Omega,
\end{aligned}\right.
$$

onde $h \in L^{\sigma}(\Omega), \sigma=2 N /(N+2), \lambda$ é um parâmetro real não-negativo e $h \geq 0$ em $\Omega$. Então $u \geq 0$ em $\Omega$. Além disso, se $h>0$ em um conjunto de medida positiva, então $u>0$ em $\Omega$.

Se $u \in C^{1}(\bar{\Omega})$, então a derivada normal exterior $\frac{\partial u}{\partial \eta}(x)<0$, para todo $x \in \Omega$.

Teorema A.5. (Princípio do Máximo Forte)(ver [16]). Sejam $\Omega \subset \mathbb{R}^{N}$ um aberto e $u \in C^{2}(\Omega) \cap C^{0}(\bar{\Omega})$ com $\Delta u \geq 0(\Delta u \leq 0)$ em $\Omega$ e suponha que existe um ponto $y \in \Omega$ tal que

$$
u(y)=\sup _{\Omega} u\left(\inf _{\Omega} u\right) .
$$

Então u é constante.

Teorema A.6. (Princípio do Máximo Fraco)(ver [16]). Sejam $\Omega \subset \mathbb{R}^{N}$ um aberto e $u \in C^{2}(\Omega) \cap C^{0}(\bar{\Omega})$ com $\Delta u \geq 0(\Delta u \leq 0)$ em $\Omega$. Então

$$
\sup _{\Omega} u=\sup _{\partial \Omega} u\left(\inf _{\Omega} u=\inf _{\partial \Omega} u\right)
$$

Definição A.2. Sejam $A \subset \Omega$ um conjunto mensurável e $f: A \rightarrow \mathbb{R}$ uma função. Se diz que $f$ é mensurável se $\{x \in A \mid f(x)>\alpha\}$ é mensurável para todo $\alpha \in \mathbb{R}$.

Definição A.3. (ver [2]). Seja $\Omega \subset \mathbb{R}^{N}, N \geq 1$. Diz-se que $f: \Omega \times \mathbb{R} \rightarrow \mathbb{R}$ é uma função de Caratheódory se:

a) $f(\cdot, s)$ é mensurável em $\Omega$, qualquer que seja $s \in \mathbb{R}$ fixado;

b) $f(x, \cdot)$ é contínua em $\mathbb{R}$ em quase todo ponto de $\Omega$. 
Teorema A.7. (Teorema da Convergência Dominada de Lebesgue)(ver [4].) Seja $\left(f_{n}\right)$ uma sequência de funções integráveis, as quais convergem em quase toda parte para uma função mensurável $f$ a valores reais. Se existe uma função integrável $g$ tal que $\left|f_{n}\right| \leq g$ para todo $n$, então $f$ é integrável e

$$
\int f d \mu=\lim \int f_{n} d \mu \text {. }
$$

Teorema A.8. (ver [17]) Sejam $f \in L^{p}(\Omega)$ com $1<p<+\infty$ e $k$ uma constante real não-negativa. Então existe uma única $u \in W^{2, p}(\Omega) \cap W_{0}^{1, p}(\Omega)$ tal que

$$
\left\{\begin{aligned}
-\Delta u+k u & =f, & & x \in \Omega, \\
u & =0, & & x \in \partial \Omega,
\end{aligned}\right.
$$

além disso, existe uma constante $C$ independente de $f$ e u tal que

$$
\|u\|_{W^{2, p}(\Omega)} \leq C\|f\|_{L^{p}(\Omega)} .
$$

Em particular, se $p>\frac{N}{2}$ e $\varphi \in C(\bar{\Omega})$, então existe uma única $u \in C(\bar{\Omega}) \cap W_{\text {loc }}^{2, p}(\Omega)$ solução do problema

$$
\left\{\begin{aligned}
-\Delta u+k u & =f, & & x \in \Omega, \\
u & =\varphi, & & x \in \partial \Omega .
\end{aligned}\right.
$$

Definição A.4. . Uma função $u: \bar{\Omega} \rightarrow \mathbb{R}$ é dita Hölder contínua de exponente $\alpha, 0<\alpha<1$, se

$$
H_{\alpha}[u]=\sup _{x \neq y} \frac{|u(x)-u(y)|}{|x-y|^{\alpha}}<\infty .
$$

Definição A.5. (Espaço $C(\bar{\Omega})$ ). Designamos por $C(\bar{\Omega})$ o espaço das funções contínuas $u: \bar{\Omega} \rightarrow \mathbb{R}$, munido da norma

$$
\|u\|_{0}=\sup _{x \in \bar{\Omega}}|u(x)| .
$$

Definição A.6 (Espaço $C^{m}(\bar{\Omega})$ ). Designamos por $C^{m}(\bar{\Omega}), m \in \mathbb{N}$, o espaço de todas as funções $u: \bar{\Omega} \rightarrow \mathbb{R}$ que juntamente com todas as derivadas de ordem inferior ou igual a $m$ são continuas em $\bar{\Omega}$. Ele é um espaço de Banach se munido com a norma

$$
\|u\|_{C^{m}(\bar{\Omega})}=\sum_{|\sigma| \leq m}\left\|D^{\sigma} u\right\|_{0}
$$

em que $\sigma=\left(\sigma_{1}, \sigma_{2}, \ldots, \sigma_{N}\right)$, com $\sigma_{i} \in \mathbb{N},|\sigma|=\sigma_{1}+\sigma_{2}+\ldots+\sigma_{N} e$

$$
D^{\sigma} u(x)=\frac{\partial^{|\sigma|} u(x)}{\partial x_{1}^{\sigma_{1}} \partial x_{2}^{\sigma_{2}} \ldots \partial x_{N}^{\sigma_{N}}} .
$$

Definição A.7 (Espaço $C^{m, \alpha}(\bar{\Omega})$ ). Designamos por $C^{m, \alpha}(\bar{\Omega})$ o espaço das funções pertencentes a $C^{m}(\bar{\Omega})$ cujas m-ésimas derivadas são Hölder contínuas de expoente $\alpha, 0<\alpha<1$, em $\bar{\Omega}$. Ele é um espaço de 
Banach se munido com a norma

$$
\|u\|_{C^{m, \alpha}(\bar{\Omega})}=\|u\|_{C^{m}(\bar{\Omega})}+\sum_{|\sigma|=m} H_{\alpha}\left[D^{\sigma} u\right] .
$$

Denotaremos $C^{0, \alpha}(\bar{\Omega})$ por $C^{\alpha}(\bar{\Omega})$ e $\|\cdot\|_{C^{0, \alpha}(\bar{\Omega})}$ por $\|\cdot\|_{C^{\alpha}(\bar{\Omega})}$.

Teorema A.9. (Estimativa de Schauder). Sejam $f \in C^{\alpha}(\bar{\Omega})$ e $u \in C^{2, \alpha}(\bar{\Omega})$ a solução única de $(A .6)$. Então existe uma constante $C$, que independe de $f$ e $u$, tal que

$$
\|u\|_{C^{2, \alpha}(\bar{\Omega})} \leq C\|f\|_{C^{\alpha}(\bar{\Omega})}, \quad \forall u \in C^{2, \alpha}(\bar{\Omega})
$$

Teorema A.10. (ver [24]) Seja $\Omega$ um dominio de classe $C^{1,1}$ em $\mathbb{R}^{N}$. Então se $h \in L^{p}(\Omega)$, com $1<$ $p<\infty$, o problema de Dirichlet $-\Delta u=h$ em $\Omega, u \in W_{0}^{1, p}(\Omega)$ tem uma única solução $u \in W^{2, p}(\Omega)$.

Teorema A.11. (Teorema de Schauder)(ver [8]). Suponha que $\Omega$ é limitado e de classe $C^{2, \alpha}$ com $0<\alpha<1$. Então para cada $f \in C^{0, \alpha}(\bar{\Omega})$ existe uma única solução $u \in C^{2, \alpha}(\bar{\Omega})$ do problema

$$
\left\{\begin{aligned}
-\Delta u+u & =f, & & x \in \Omega, \\
u & =0, & & x \in \partial \Omega .
\end{aligned}\right.
$$

Proposição A.1. Seja $\Omega \subset \mathbb{R}^{N}$ um aberto limitado, denote $H=H(\Omega)=H_{0}^{1}(\Omega)$ e seja $p \in[1,+\infty)$ tal que $p<2^{*}$ se $N \geq 3$. Se $\left(u_{n}\right) \subset H$ é uma sequência limitada, então existe uma subsequência $\left(u_{n_{j}}\right) \subseteq\left(u_{n}\right)$ tal que

$$
\begin{aligned}
& u_{n_{j}} \rightarrow u \text { fracamente em } H \\
& u_{n_{j}} \rightarrow u \text { fortemente em } L^{p}(\Omega) \\
& u_{n_{j}} \rightarrow u \text { em quase todo ponto de } \Omega .
\end{aligned}
$$

Considerando agora o funcional da forma

$$
\Phi(u)=\frac{1}{2} \int_{\Omega}|\nabla u|^{2} d x-\int_{\Omega} F(x, u) d x
$$

definido sobre $H$, em que $\Omega \subset \mathbb{R}^{N}$ é um domínio suave e limitado, e

$$
F(x, u)=\int_{0}^{u} f(x, s) d s
$$

Supondo $f$ mensurável em $x$, contínua em $u$ e verificando

$$
|f(x, u)| \leq c\left(1+|u|^{p}\right) \quad \text { com } \quad p \leq \frac{N+2}{N-2} .
$$

Teorema A.12. (Ver [7]). Suponha $u_{0} \in H$ é um minimo local de $\Phi$ na topologia de $C^{1}$, isto é existe algum $r>0$ tal que

$$
\Phi\left(u_{0}\right) \leq \Phi\left(u_{0}+v\right), \forall v \in C_{0}^{1}(\bar{\Omega}) \operatorname{com}\|v\|_{C^{1}} \leq r .
$$

Então $u_{0}$ é um minimo local de $\Phi$ na topologia de $H$, isto é existe $\varepsilon_{0}>0$ tal que

$$
\Phi\left(u_{0}\right) \leq \Phi\left(u_{0}+v\right), \forall v \in H_{0}^{1}(\bar{\Omega}) \operatorname{com}\|v\|_{H^{1}} \leq \varepsilon_{0}
$$


Teorema A.13 (Desigualdade de Hardy). Dado $p>1$ e $f \in L^{p}(0, \infty), f \geq 0$ então

$$
\int_{0}^{\infty}\left(\frac{1}{x} \int_{0}^{x} f(t) d t\right)^{p} d x \leq\left(\frac{p}{p-1}\right)^{p} \int_{0}^{\infty} f(x)^{p} d x
$$

\section{A.1 Regularidade}

Vamos agora estabelecer a regularidade $C^{2, \alpha}$ das soluções fracas de $\left(P_{\lambda}\right)$ pertencentes a $H^{1}(\Omega)$.

Lema A.2. Seja $v \in H^{1}(\Omega)$ uma solução do problema $\left(P_{\lambda}\right)$, então $v \in C^{2, \alpha}(\Omega)$ para algum $\alpha \in(0,1)$.

Demonstração. Seja $v \in H^{1}(\Omega)$ uma solução de $\left(P_{\lambda}\right)$ fixado. E denotemos

$$
f_{\lambda}(x)=f_{\lambda}(x, v(x))=\lambda\left(v^{+}(x)\right)^{q}+\left(v^{+}\right)^{p} .
$$

Pela imersão contínua $H^{1}(\Omega) \hookrightarrow L^{r}(\Omega)$ para $r=2^{*}$, como $v \in H^{1}(\Omega)$, então $v \in L^{r}(\Omega)$. Daí

$$
\int_{\Omega}\left(v^{+}\right)^{q \frac{r}{p}}=\int_{\left\{\left(v^{+}\right) \leq 1\right\}}\left(v^{+}\right)^{q \frac{r}{p}} d x+\int_{\left\{\left(v^{+}\right) \geq 1\right\}}\left(v^{+}\right)^{q \frac{r}{p}} d x \leq|\Omega|+\int_{\Omega}\left(v^{+}\right)^{r} d x<\infty .
$$

Logo,

$$
\left(\int_{\Omega}\left|f_{\lambda}(x)\right|^{r / p} d x\right)^{p / r}<\infty
$$

ou seja, $f_{\lambda}(x) \in L^{\vartheta}(\Omega)$, para $\vartheta=\frac{r}{p}$. Como $1<p<2^{*}-1$, obtemos

$$
\vartheta=\frac{r}{p}>\frac{2^{*}}{2^{*}-1}
$$

Assim, existe $\varepsilon>0$ tal que

$$
\vartheta=\frac{2^{*}}{2^{*}-1}(1+\varepsilon)
$$

Podemos escrever o problema como uma equação elíptica linear não homogênea.

$$
\left\{\begin{aligned}
-\Delta v & =f_{\lambda}(x), & & x \in \Omega, \\
v & =0, & & x \in \partial \Omega .
\end{aligned}\right.
$$

De acordo como o Teorema de Regularidade para equações elípticas lineares não homogêneas (ver Apêndice $A$ Teorema A.10), como $f_{\lambda} \in L^{\vartheta}(\Omega)$, temos que $v \in W^{2, \vartheta}(\Omega)$.

Se $2 \vartheta>N$, pelas imersões de Sobolev temos que $W^{2, \vartheta}(\Omega) \hookrightarrow C^{0, \vartheta}(\bar{\Omega})$. Sendo $v \in C^{0, \vartheta}(\Omega)$, assim $f_{\lambda}(x, v) \in C^{0, \vartheta}(\bar{\Omega})$. Logo pelo Teorema (ver Teorema A.11) temos que $v \in C^{2, \vartheta}(\bar{\Omega})$.

Se $2 \vartheta=N$, então temos que $W_{0}^{2, \vartheta}(\Omega) \hookrightarrow L^{\gamma}(\Omega)$, para todo $\gamma>\vartheta$. Logo, tomando $\frac{\gamma}{p}>\frac{N}{2}$ temos que $v \in L^{\gamma}(\Omega)$. Claramente, vemos que $f_{\lambda} \in L^{\xi}(\Omega)$, para $\psi=\frac{\gamma}{p}$ então pelo Teorema $A .10$, temos $v \in W^{2, \xi}(\bar{\Omega})$. Pelas imersões de Sobolev, $v \in C^{0, \xi}(\bar{\Omega})$, assim $f_{\lambda} \in C^{0, \vartheta}(\bar{\Omega})$. Daí pelo teorema de Schauder (ver Teorrema A.11) segue que $v \in C^{2, \xi}(\bar{\Omega})$.

No caso em que $2 \vartheta<N$, pelo Teorema de Imersões de Sobolev, temos

$$
W^{2, \vartheta}(\Omega) \hookrightarrow L^{r_{1}}(\Omega),
$$


$\operatorname{com} r_{1}=\frac{N \vartheta}{N-2 \vartheta}$. Como $v \in W^{2, \vartheta}(\Omega)$, então $v \in L^{r_{1}}(\Omega)$ e tem-se

$$
\int_{\Omega}\left(v^{+}\right)^{q \frac{r_{1}}{p}}=\int_{\left\{\left(v^{+}\right) \leq 1\right\}}\left(v^{+}\right)^{q \frac{r_{1}}{p}} d x+\int_{\left\{\left(v^{+}\right) \geq 1\right\}}\left(v^{+}\right)^{q \frac{r_{1}}{p}} d x \leq|\Omega|+\int_{\Omega}\left(v^{+}\right)^{r_{1}} d x<\infty
$$

e resulta que

$$
\left(\int_{\Omega}\left|f_{\lambda}(x)\right|^{r_{1} / p} d x\right)^{p / r_{1}}<\infty .
$$

Logo $f_{\lambda}(x) \in L^{\vartheta_{1}}(\Omega), \operatorname{com} \vartheta_{1}=\frac{r_{1}}{p}$. Então, $v \in W^{2, \vartheta_{1}}(\Omega)$.

Para mostrar que a regularidade de $v$ foi melhorada, é necessário mostrar que

$$
\frac{\vartheta_{1}}{\vartheta}=\frac{r_{1}}{r}>1
$$

Temos que

$$
r_{1}=\frac{N\left(\frac{2^{*}}{2^{*}-1}(1+\varepsilon)\right)}{N-2\left(\frac{2^{*}}{2^{*}-1}\right)(1+\varepsilon)}=\frac{2^{*} N(1+\varepsilon)}{N\left(2^{*}-1\right)-2\left(2^{*}\right)(1+\varepsilon)} .
$$

Daí

$$
\frac{r_{1}}{r}=\frac{2^{*} N(1+\varepsilon)}{N\left(2^{*}-1\right)-2\left(2^{*}\right)(1+\varepsilon)} \cdot \frac{1}{2^{*}}=\frac{N(1+\varepsilon)}{N\left(2^{*}-1\right)-2\left(2^{*}\right)(1+\varepsilon)} .
$$

Assim, basta verificarmos que

$$
N\left(2^{*}-1\right)-2(1+\varepsilon) 2^{*}>0
$$

e

$$
N(1+\varepsilon)>N\left(2^{*}-1\right)-2\left(2^{*}\right)(1+\varepsilon) .
$$

A partir de (A.8), temos

$$
N\left(2^{*}-1\right)>2(1+\varepsilon) 2^{*}
$$

o que implica que

$$
\frac{N\left(2^{*}-1\right)}{2\left(2^{*}\right)}-1>\varepsilon
$$

e por $(A .9)$, temos

$$
\begin{aligned}
N\left(2^{*}-1\right) & <N(1+\varepsilon)+2(1+\varepsilon) 2^{*} \\
& =\left(N+2\left(2^{*}\right)\right)(1+\varepsilon),
\end{aligned}
$$

daí

$$
\frac{N\left(2^{*}-1\right)}{N+2\left(2^{*}\right)}-1<\varepsilon
$$

Assim,

$$
\frac{N\left(2^{*}-1\right)}{N+2\left(2^{*}\right)}-1<\varepsilon<\frac{N\left(2^{*}-1\right)}{2\left(2^{*}\right)}-1 .
$$

Podemos encontrar um $\varepsilon>0$ satisfazendo $(A .11)$, consequentemente, temos

$$
\frac{\vartheta_{1}}{\vartheta}>1 \text {. }
$$

Pelo Teorema de Regularidade (ver Teorema A.10), assim como para $\vartheta_{1}>\vartheta$ temos $v \in W^{2, \vartheta_{1}}(\Omega)$, 
também para qualquer $\vartheta_{k}$ suficientemente grande, $v \in W^{2, \vartheta_{k}}(\Omega)$. Quando $2 \vartheta_{k}>N$, pelo Teorema de Imersão de Sobolev podemos ter que $v \in C^{0, \vartheta}(\bar{\Omega}), f_{\lambda} \in C^{0, \vartheta}(\bar{\Omega})$, então $v \in C^{2, \vartheta}(\bar{\Omega})$ pelo Teorema de Schauder, ver Teorema A.11.

\section{A.2 $I_{\lambda}$ satisfaz a condição $(P S)$}

Consideramos o problema

$$
\left(P_{\lambda}\right) \quad\left\{\begin{aligned}
-\Delta u & =\lambda u^{q}+u^{p}, & & x \in \Omega, \\
u & =0, & & x \in \partial \Omega,
\end{aligned}\right.
$$

onde $\Omega$ é um aberto limitado, contido em $\mathbb{R}^{N}$, com $0<q<1<p<2^{*}-1$ e $\lambda$ um parâmetro real. O funcional associado ao problema $\left(P_{\lambda}\right)$ é

$$
I_{\lambda}(u)=\frac{1}{2}\|u\|^{2}-\frac{\lambda}{q+1} \int_{\Omega} u^{q+1} d x-\frac{1}{p+1} \int_{\Omega} u^{p+1} d x .
$$

Lema A.3. $I_{\lambda}$ satisfaz a condição $(P S)_{c}$.

Demonstração. Mostraremos que toda sequência $(P S)$ possui subsequência convergente.

Seja $\left(v_{n}\right) \subset H(\Omega)$ uma sequência $(P S)$, isto é,

$$
I_{\lambda}\left(v_{n}\right) \rightarrow c, \quad \text { e } \quad I_{\lambda}^{\prime}\left(v_{n}\right) \rightarrow 0 \text { em } H^{-1}(\Omega) .
$$

Por $(A .12)$ podemos assumir que $\left(v_{n}\right)$ satisfaz

$$
\frac{1}{2} \int_{\Omega}\left|\nabla v_{n}\right|^{2} d x-\frac{\lambda}{q+1} \int_{\Omega}\left(v_{n}^{+}\right)^{q+1} d x-\frac{1}{p+1} \int_{\Omega}\left(v_{n}^{+}\right)^{p+1} d x=c+o_{n}(1) .
$$

Agora, pela definição de $I_{\lambda}^{\prime}$ temos

$$
\int_{\Omega}\left|\nabla v_{n}\right|^{2} d x=I_{\lambda}^{\prime}\left(v_{n}\right) v_{n}+\lambda \int_{\Omega}\left(v_{n}^{+}\right)^{q+1} d x+\int_{\Omega}\left(v_{n}^{+}\right)^{p+1} d x .
$$

Sustituindo (A.14) em (A.13) obtemos

$$
\begin{aligned}
c+o_{n}(1) & =\frac{1}{2} I_{\lambda}^{\prime}\left(v_{n}\right) v_{n}+\frac{\lambda}{2} \int_{\Omega}\left(v_{n}^{+}\right)^{q+1} d x+\frac{1}{2} \int_{\Omega}\left(v_{n}^{+}\right)^{p+1} d x \\
& -\frac{\lambda}{q+1} \int_{\Omega}\left(v_{n}^{+}\right)^{q+1} d x-\frac{1}{p+1} \int_{\Omega}\left(v_{n}^{+}\right)^{p+1} d x,
\end{aligned}
$$

assim

$$
\begin{aligned}
\lambda\left(\frac{1}{2}-\frac{1}{q-1}\right) \int_{\Omega}\left(v_{n}^{+}\right)^{q+1} d x+\left(\frac{1}{2}-\frac{1}{p+1}\right) \int_{\Omega}\left(v_{n}^{+}\right)^{p+1} d x & =c-\frac{1}{2} I_{\lambda}^{\prime}\left(v_{n}\right) u_{n}+o_{n}(1) \\
\frac{\lambda(q-1)}{2(q+1)} \int_{\Omega}\left(v_{n}^{+}\right)^{q+1} d x+\frac{p-1}{2(p+1)} \int_{\Omega}\left(v_{n}^{+}\right)^{p+1} d x & =c-\frac{1}{2} I_{\lambda}^{\prime}\left(v_{n}\right) v_{n}+o_{n}(1) .
\end{aligned}
$$


Seja $c_{1}=\frac{p-1}{2(p+1)}$ e $c_{2}=\frac{\lambda(1-q)}{2(p+1)}$. Como $0<q<1<p$, vemos claramente que $c_{1}, c_{2}>0$. Assim,

$$
c_{1} \int_{\Omega}\left(v_{n}^{+}\right)^{p+1} d x \leq c_{2} \int_{\Omega}\left(v_{n}^{+}\right)^{q+1} d x+C+\left\|I_{\lambda}^{\prime}\left(v_{n}\right)\right\|_{H^{-1}}\left\|v_{n}\right\|,
$$

isto é,

$$
\left\|v_{n}^{+}\right\|_{p+1}^{p+1} \leq C\left\|v_{n}^{+}\right\|_{q+1}^{q+1}+C+C\left\|I_{\lambda}^{\prime}\left(v_{n}\right)\right\|_{H^{-1}}\left\|v_{n}\right\|
$$

Combinando (A.13) e (A.15), temos

$$
\begin{aligned}
\frac{1}{2}\left\|v_{n}\right\|^{2} & =\frac{\lambda}{q+1}\left\|v_{n}^{+}\right\|_{q+1}^{q+1}+\frac{1}{p+1}\left\|v_{n}^{+}\right\|_{p+1}^{p+1}+C+o_{n}(1) \\
& \leq \frac{\lambda}{q+1}\left\|v_{n}^{+}\right\|_{q+1}^{q+1}+\frac{1}{p+1}+\left(C\left\|v_{n}^{+}\right\|_{q+1}^{q+1}+C+C\left\|I_{\lambda}^{\prime}\left(v_{n}\right)\right\|_{H^{-1}}\left\|v_{n}\right\|\right)+C \\
& =C\left\|v_{n}^{+}\right\|_{q+1}^{q+1}+C+C\left\|I_{\lambda}^{\prime}\left(v_{n}\right)\right\|_{H^{-1}}\left\|v_{n}\right\| .
\end{aligned}
$$

Pela imersão compacta de $H \hookrightarrow L^{q+1}(\Omega)$, temos que

$$
\left\|v_{n}\right\|^{2} \leq C\left\|v_{n}^{+}\right\|^{q+1}+C+C\left\|v_{n}\right\| .
$$

Deduzimos então que existe uma constante $C$ tal que $\left\|v_{n}\right\| \leq C$. Consequentemente, $\left(v_{n}\right)$ é limitada em $H$ e então existe uma subsequência $\left(v_{n_{j}}\right) \subset\left(v_{n}\right)$ fracamente convergente em $H$. Além disso, temos uma outra desigualdade, $\left\|v_{n}^{+}\right\|_{p+1}^{p+1} \leq C$, que é dada pela imersão $H(\Omega) \hookrightarrow L^{p+1}(\Omega)$.

Assim, podemos deduzir o seguinte

$$
\begin{array}{ll}
v_{n_{j}} \rightarrow v & \text { fortemente em } L^{p+1}(\Omega) \quad \text { para } 2<p+1<2^{*} ; \\
v_{n_{j}} \rightarrow v & \text { fortemente em } L^{q+1}(\Omega) \quad \text { para } 1<q+1<p+1 \\
v_{n_{j}} \rightarrow v & \text { em quase todo ponto de } \Omega
\end{array}
$$

Considere $P_{j}, P_{0}: H \rightarrow \mathbb{R}$ dados por

$$
P_{j}(\varphi)=\lambda \int_{\Omega}\left(v_{n_{j}}^{+}\right)^{q} \varphi d x+\int_{\Omega}\left(v_{n_{j}}^{+}\right)^{p} \varphi d x
$$

e

$$
P_{0}(\varphi)=\lambda \int_{\Omega}\left(v^{+}\right)^{q} \varphi d x+\int_{\Omega}\left(v^{+}\right)^{p} \varphi d x
$$


Assim,

$$
\begin{aligned}
\mid\left(P_{j}-P_{0} \mid\right) & =\left|\lambda \int_{\Omega}\left(\left(v_{n_{j}}^{+}\right)^{q}-\left(v^{+}\right)^{q}\right) \varphi d x+\int_{\Omega}\left(\left(v_{n_{j}}^{+}\right)^{p}-\left(v^{+}\right)^{p}\right) \varphi d x\right| \\
& \leq\left(\int_{\Omega}\left(\left(v_{n_{j}}^{+}\right)^{q}-\left(v^{+}\right)^{q}\right)^{\frac{q+1}{q}} d x\right)^{\frac{q}{q+1}}\left(\int_{\Omega}|\varphi|^{q+1} d x\right)^{\frac{1}{q+1}} \\
& +\left(\int_{\Omega}\left(\left(v_{n_{j}}^{+}\right)^{p}-\left(v^{+}\right)^{p}\right)^{\frac{p+1}{p}} d x\right)^{\frac{p}{p+1}}\left(\int_{\Omega}|\varphi|^{p+1} d x\right)^{\frac{1}{p+1}} \\
& \leq c\|\varphi\|\left(\int_{\Omega}\left(\left(v_{n_{j}}^{+}\right)^{q}-\left(v^{+}\right)^{q}\right)^{\frac{q+1}{q}} d x\right)^{\frac{q}{q+1}} \\
& +c\|\varphi\|\left(\int_{\Omega}\left(\left(v_{n_{j}}^{+}\right)^{p}-\left(v^{+}\right)^{p}\right)^{\frac{p+1}{p}} d x\right)^{\frac{p}{p+1}} .
\end{aligned}
$$

Como $v_{n_{j}} \rightarrow v$ em quase todo ponto de $\Omega$ vemos que $v_{n_{j}}^{+} \rightarrow v^{+}$em quase todo ponto de $\Omega$ e como $v_{n_{j}} \rightarrow v$ em $L^{q+1}(\Omega)$, então existe $l \in L^{q+1(\Omega)}$ tal que

$$
v_{n_{j}}^{+} \leq\left|v_{n_{j}}(x)\right| \leq l(x) \quad \text { em quase todo ponto de } \Omega
$$

Assim,

$$
\begin{aligned}
\left(\left(v_{n_{j}}^{+}\right)^{q}-\left(v^{+}\right)^{q}\right)^{\frac{q+1}{q}} & \leq\left(\left(v_{n_{j}}^{+}\right)^{q}+\left(v^{+}\right)^{q}\right)^{\frac{q+1}{q}} \leq c\left(\left(v_{n_{j}}^{+}\right)^{q+1}+\left(v^{+}\right)^{q+1}\right) \\
& \left.\leq c(|l(x)|)^{q+1}+\left(v^{+}\right)^{q+1}\right) \in L^{1}(\Omega) .
\end{aligned}
$$

Pelo Teorema da Convergência Dominada de Lebesgue, temos

$$
\int_{\Omega}\left(\left(v_{n_{j}}^{+}\right)^{q}-\left(v^{+}\right)^{q}\right)^{\frac{q+1}{q}} d x \rightarrow 0 .
$$

Por outro lado, como $v_{n} \rightarrow v$ em $L^{p+1}(\Omega)$, então existe $l_{1} \in L^{p+1}(\Omega)$ tal que

$$
\left|v_{n_{j}}(x)\right| \leq l_{1}(x) \quad \text { em quase todo ponto de } \Omega
$$

Daí,

$$
\begin{aligned}
\left(\left(v_{n_{j}}\right)^{p}-\left(v^{+}\right)^{p}\right)^{\frac{p+1}{p}} & \leq C\left(\left(v_{n_{j}}\right)^{p}+\left(v^{+}\right)^{p}\right) \\
& \leq C\left(l_{1}^{p+1}(x)+|v|^{p+1}\right) \in L^{1}(\Omega) .
\end{aligned}
$$

Logo, novamente pelo Teorema da Convergência Dominada de Lebesgue, temos

$$
\int_{\Omega}\left(\left(v_{n_{j}}^{+}\right)^{p}-\left(v^{+}\right)^{p}\right)^{\frac{p+1}{p}} d x \rightarrow 0 .
$$

Portanto, $P_{j} \rightarrow P_{0}$ em $H^{-1}(\Omega)$, ou ainda

$$
\lambda\left(v_{n_{j}}^{+}\right)^{q}+\left(v_{n_{j}}^{+}\right)^{p} \rightarrow \lambda\left(v^{+}\right)^{q}+\left(v^{+}\right)^{p} \quad \text { em } H^{-1}(\Omega)
$$

Pelo Teorema de Representação de Riesz, para cada $f_{\lambda}(w)=\lambda\left(w^{+}\right)^{q}+\left(w^{+}\right)^{p}$ em $H^{-1}(\Omega)$, o problema

$$
(\tilde{P}) \quad\left\{\begin{aligned}
-\Delta u & =f_{\lambda}(w), & & x \in \Omega, \\
u & =0, & & x \in \partial \Omega,
\end{aligned}\right.
$$


tem uma única solução $u \in H^{1}(\Omega)$.

Considere $\Phi: H^{-1} \rightarrow H^{1}$, e seja $u=\Phi\left(f_{\lambda}(w)\right)$. Notamos que $\Phi$ é uma isometria, pois é um isomorfismo que preserva o produto interno.

Como $u$ é a única solução do problema $(\tilde{P})$, então satisfaz

$$
\int_{\Omega} f_{\lambda}(w) \varphi d x=\int_{\Omega} \nabla u \nabla \varphi d x=\langle u, \varphi\rangle=\left\langle\Phi\left(f_{\lambda}(w), \varphi\right\rangle,\right.
$$

para todo $\varphi \in H(\Omega)$. Assim,

$$
\begin{aligned}
\left\langle I_{\lambda}^{\prime}(w), \varphi\right\rangle & =\langle w, \varphi\rangle-\int_{\Omega} f_{\lambda}(w) \varphi d x \\
& =\langle w, \varphi\rangle-\left\langle\Phi\left(f_{\lambda}(w), \varphi\right\rangle\right. \\
& =\left\langle w-\Phi\left(f_{\lambda}(w), \varphi\right\rangle .\right.
\end{aligned}
$$

Logo,

$$
I_{\lambda}^{\prime}(w)=w-\Phi\left(f_{\lambda}(w)\right)
$$

Sendo $\Phi$ contínua, temos que

$$
\Phi\left(f_{\lambda}\left(v_{n_{j}}\right)\right) \rightarrow \Phi\left(f_{\lambda}(v) \quad \text { em } H\right.
$$

Por $(A .12)$ temos que

$$
I_{\lambda}^{\prime}\left(v_{n_{j}}\right)=v_{n_{j}}-\Phi\left(f_{\lambda}\left(v_{n_{j}}\right)\right) \rightarrow 0 \quad \text { em } H^{-1}(\Omega),
$$

consequentemente,

$$
v_{n_{j}} \rightarrow \Phi\left(f_{\lambda}(v)\right) \quad \text { em } H .
$$

Pela unicidade do limite fraco, temos que $v=\Phi\left(f_{\lambda}(v)\right)$, mas $u=\Phi\left(f_{\lambda}(v)\right)$. Assim $v=u$. E portanto, $I_{\lambda}$ satisfaz a condição $(P S)$.

\section{A.3 A constante de Sobolev}

Para darmos prosseguimento ao nosso estudo, introduziremos os seguintes espaços

$$
\mathcal{D}^{1,2}(\Omega)=\left\{u \in L^{2^{*}}(\Omega): \nabla u \in L^{2}(\Omega)\right\},
$$

onde $\mathcal{D}_{0}^{1,2}(\Omega)$ é o fecho, na métrica da convergência uniforme, do conjunto das funções de suporte compacto contido em $\Omega$. No decorrer de nosso trabalho será de muita importância a utilização da constante de Sobolev

$$
S=\inf _{\substack{u \in \mathcal{D}^{1,2}\left(\mathbb{R}^{N}\right) \\\|u\|^{2^{*}}=1}}\|\nabla u\|^{2} .
$$

O Teorema $A .14$ que apresentaremos a seguir, nos garante que a constante $S$ é atingida. Logo existe $u \in \mathcal{D}^{1,2}\left(\mathbb{R}^{N}\right)$ tal que

$$
\int_{\mathbb{R}^{N}}|\nabla u|^{2} d x=S \quad \text { e } \quad\|u\|_{2^{*}}=1 .
$$


Pode-se mostrar, que as funções que realizam esse mínimo estão relacionadas com a função

$$
v(x)=\frac{C_{N}}{\left(1+|x|^{2}\right)^{(N-2) / 2}},
$$

em que $C_{N}=[N(N-2)]^{(N-2) / 4}$.

A função acima é tal que

$$
\int_{\mathbb{R}^{N}}|\nabla v|^{2} d x=S^{N / 2}=\int_{\mathbb{R}^{N}}|v|^{2^{*}} d x
$$

Desse modo, se $u=\frac{v}{\|v\|^{2^{*}}}$ então $\|u\|_{2^{*}}=1 \mathrm{e}$

$$
\int_{\mathbb{R}^{N}}|\nabla u|^{2} d x=S
$$

Lema A.4. (Concentração de Compacidade) Seja $\left(u_{n}\right) \subset \mathcal{D}^{1,2}\left(\mathbb{R}^{N}\right)$ uma sequência tal que

$$
\begin{aligned}
u_{n} \rightarrow u & \text { em } \mathcal{D}^{1,2}\left(\mathbb{R}^{N}\right), \\
\left|\nabla\left(u_{n}-u\right)\right|^{2} \rightarrow \mu & \text { em } \mathcal{M}\left(\mathbb{R}^{N}\right), \\
\left|u_{n}-u\right|^{2^{*}} \rightarrow \nu & \text { em } \mathcal{M}\left(\mathbb{R}^{N}\right), \\
u_{n} \rightarrow u & \text { em quase todo ponto de } \mathbb{R}^{N} .
\end{aligned}
$$

Defina

$$
\mu_{\infty}=\lim _{R \rightarrow \infty} \varlimsup_{n \rightarrow \infty} \int_{|x|>R}\left|\nabla u_{n}\right|^{2} d x, \quad \nu_{\infty}=\lim _{R \rightarrow \infty} \varlimsup_{n \rightarrow \infty} \int_{|x|>R}\left|u_{n}\right|^{2^{*}} d x
$$

Então

$$
\begin{aligned}
\|\nu\|^{2 / 2^{*}} & \leq S^{-1}\|\mu\|, \\
\nu_{\infty}^{2 / 2^{*}} & \leq S^{-1} \mu_{\infty}, \\
\varlimsup_{n \rightarrow \infty}\left|\nabla u_{n}\right|_{2}^{2} & =|\nabla u|_{2}^{2}+\|\mu\|+\mu_{\infty}, \\
\varlimsup_{n \rightarrow \infty}\left|u_{n}\right|_{2^{*}}^{2^{*}} & =|u|_{2^{*}}^{2^{*}}+\|\nu\|+\nu_{\infty} .
\end{aligned}
$$

Além disso, se $u=0$ e $\|\nu\|^{2 / 2^{*}}=S^{-1}\|\mu\|$, então cada uma das medidas $\mu$ e $\nu$ se concentram em um único ponto.

Teorema A.14. Seja $\left(u_{n}\right) \subset \mathcal{D}^{1,2}\left(\mathbb{R}^{n}\right)$ uma sequência tal que

$$
\left\|u_{n}\right\|_{2^{*}}=1 \quad e \quad \lim _{n \rightarrow \infty}\left\|\nabla u_{n}\right\|_{2}^{2}=S
$$

Então existe $\left(y_{n}, \lambda_{n}\right) \subset \mathbb{R}^{N} \times(0, \infty)$ tal que a sequência

$$
v_{n}(x)=\lambda_{n}^{(N-2) / 2} u_{n}\left(\lambda_{n} x+y_{n}\right)
$$

possui subsequência convergente. Observe que se $u \in \mathcal{D}^{1,2}\left(\mathbb{R}^{N}\right),(y, \lambda) \in \mathbb{R}^{N} \times(0, \infty)$ e $v(x)=\lambda^{(N-2) / 2} u(\lambda x+$ y) então

$$
\|v\|_{2^{*}}=\|u\|_{2^{*}} \quad e \quad\|\nabla u\|_{2}=\|\nabla v\|_{2}
$$


Demonstração. Definamos

$$
P_{n}(\lambda)=\sup _{y \in \mathbb{R}^{N}} \int_{B_{\lambda}(y)}\left|u_{n}\right|^{2^{*}}
$$

Como

$$
\lim _{\lambda \rightarrow 0^{+}} P_{n}(\lambda)=0, \lim _{\lambda \rightarrow \infty} P_{n}(\lambda)=1
$$

existe $\lambda_{n}>0$ tal que $P_{n}\left(\lambda_{n}\right)=\frac{1}{2}$. Além disso existe $y_{n} \in \mathbb{R}^{N}$ tal que

$$
\int_{B_{\lambda_{n}}\left(y_{n}\right)}\left|u_{n}\right|^{2^{*}}=P_{n}\left(\lambda_{n}\right)=\frac{1}{2}
$$

Isto ocorre porque existe uma sequência $\left(y_{k, n}\right) \subset \mathbb{R}^{N}$ tal que

$$
\lim _{k \rightarrow \infty} \int_{B_{\lambda_{n}}\left(y_{k, n}\right)}\left(y_{k, n}\right)\left|u_{n}\right|^{2^{*}}=P_{n}\left(\lambda_{n}\right) .
$$

Tal sequência não pode ser ilimitada pois

$$
\lim _{|y| \rightarrow \infty} \int_{B_{\lambda_{n}}(y)}\left|u_{n}\right|^{2^{*}}=0
$$

Logo, existe um valor $y_{n}$ tal que, a menos de subsequência, $y_{k, n} \rightarrow y_{n}$ quando $k \rightarrow \infty$. Usando o Teorema da Convergência Dominada de Lebesgue obtemos

$$
\int_{B_{\lambda_{n}}\left(y_{n}\right)}\left|u_{n}\right|^{2^{*}}=P_{n}\left(\lambda_{n}\right)=\frac{1}{2}
$$

Defina $v_{n}(x)=u_{n}^{y_{n}, \lambda_{n}}(x)$, temos $\left\|v_{n}\right\|_{2^{*}}=\left\|u_{n}\right\|_{2^{*}}=1, \lim _{n \rightarrow \infty}\left\|\nabla v_{n}\right\|_{2}^{2}=\left\|\nabla u_{n}\right\|_{2}^{2}=S$ e após de uma mudança de variável temos

$$
\frac{1}{2}=\int_{B_{1}(0)}\left|v_{n}\right|^{2^{*}}=\sup _{y \in \mathbb{R}^{N}} \int_{B_{1}(y)}\left|v_{n}\right|^{2^{*}} .
$$

Sendo $\left(v_{n}\right)$ limitada em $\mathcal{D}^{1,2}\left(\mathbb{R}^{N}\right)$, existe $v \in \mathcal{D}^{1,2}\left(\mathbb{R}^{N}\right)$ tal que a menos de subsequências

$$
\begin{aligned}
v_{n} \rightarrow v & \text { em } \mathcal{D}^{1,2}\left(\mathbb{R}^{N}\right), \\
\left|\nabla\left(v_{n}-v\right)\right|^{2} \rightarrow \mu & \text { em } \mathcal{M}\left(\mathbb{R}^{N}\right), \\
\left|v_{n}-v\right|^{2^{*}} \rightarrow \nu & \text { em } \mathcal{M}\left(\mathbb{R}^{N}\right), \\
v_{n} \rightarrow v & \text { em quase todo ponto de } \mathbb{R}^{N} .
\end{aligned}
$$

Mostraremos agora que $v$ atinge o valor $S$. Do Lema (A.4) temos que

$$
\begin{gathered}
S=\varlimsup_{n \rightarrow \infty}\left\|\nabla v_{n}\right\|_{2}^{2}=\|\nabla u\|_{2}^{2}+\|\mu\|+\mu_{\infty}, \\
1=\varlimsup_{n \rightarrow \infty}\left\|u_{n}\right\|_{2^{*}}^{2^{*}}=\|u\|_{2^{*}}^{2^{*}}+\|\nu\|+\nu_{\infty}, \\
\|\nu\|^{2 / 2^{*}} \leq S^{-1}\|\mu\|, \nu_{\infty}^{2 / 2^{*}} \leq S^{-1} \mu_{\infty} .
\end{gathered}
$$

Afirmamos que cada valor $\|v\|_{2^{*}}^{2^{*}},\|\nu\|$ e $\nu_{\infty}$ é igual a 0 ou 1 . De fato, se algum destes valores 
estivesse no intervalo $(0,1)$, poderíamos usar a desigualdade

$$
(a+b)^{t}<a^{t}+b^{t}, a, b>0, \quad 0<t<1,
$$

concluindo que

$$
1=\left(\|v\|_{2^{*}}+\|\nu\|+\nu_{\infty}\right)^{2 / 2^{*}}<\|v\|_{2^{*}}^{2}+\|\nu\|^{2 / 2^{*}}+\nu_{\infty}^{2 / 2^{*}} .
$$

Da desigualdade acima, definição de $S$ e as equações $(A .28)$ e (A.29) implicam que

$$
S<S\left(\|v\|_{2^{*}}^{2}+\|\nu\|^{2 / 2^{*}}+\nu_{\infty}^{2 / 2^{*}}\right) \leq\|\nabla v\|_{2}^{2}+\|\mu\|+\mu_{\infty}=S .
$$

O que é absurdo. Logo $\|v\|_{2^{*}}^{2^{*}},\|\nu\|, \nu_{\infty} \in\{0,1\rangle$. A equação (A.26) implica que

$$
\varlimsup_{n \rightarrow \infty} \int_{|x|>1}\left|v_{n}\right|^{2 *}=\frac{1}{2} .
$$

Como

$$
\int_{|x|>R}\left|v_{n}\right|^{2 *} \leq \int_{|x|>1}\left|v_{n}\right|^{2 *}, \quad \text { se } R>1,
$$

então, $\nu_{\infty} \leq \frac{1}{2}, \operatorname{logo} \nu_{\infty}=0$. Se $\|\nu\|=1$ então $\|v\|_{2^{*}}^{2^{*}}=0$ e assim $v=0$, logo

$$
\|\nu\|^{2 / 2^{*}} \geq S^{-1}\|\mu\| \text {. }
$$

De (A.29) concluímos que

$$
\|\nu\|^{2 / 2^{*}}=S\|\mu\| .
$$

Como $v=0$, segue que a medida $\nu$ está concentrada em um único ponto $z \in \mathbb{R}^{N}$. De (A.26) obtemos que

$$
\frac{1}{2}=\sup _{y \in \mathbb{R}^{N}} \int_{B_{1}(y)}\left|v_{n}\right|^{2^{*}} \geq \int_{B_{1}(z)}\left|v_{n}\right|^{2^{*}}=\lim _{n \rightarrow \infty} \int_{B_{1}(z)} d \nu=\|v\|=1 .
$$

E isto é uma contradição. Assim, $\|v\|_{2^{*}}^{2^{*}}=1$ e portanto $\|\nu\|=0, \nu_{\infty}=0$. De (A.28) concluímos que

$$
\|\nabla v\|_{2}^{2}=S=\lim _{n \rightarrow \infty}\left\|\nabla v_{n}\right\|_{2}^{2}
$$

Lema A.5. As seguintes desigualdades são verdadeiras:

1. Existe uma constante $C(p)>0$ tal que

$$
\frac{(r+s)^{p+1}-r^{p+1}}{p+1}-r^{p} \geq \frac{s^{p+1}}{p+1}+C r^{p-1} s^{2}, \quad r, s \geq 0, p>1 .
$$

2. Para $r, s \geq 0,0<q<1$, afirmamos que existe uma constante $C(q)>0$ tal que

$$
\frac{(r+s)^{q+1}-r^{q+1}}{q+1}-r^{q} s \leq C(q) s^{q+1} .
$$


Demonstração. 1. Mostraremos que, para todo $p>1$ existe uma constante $C=C(p)>0$ tal que

$$
\begin{gathered}
\frac{(r+s)^{p+1}-r^{p+1}}{p+1}-r^{p} \geq \frac{s^{p+1}}{p+1}+C r^{p-1} s^{2}, \quad r, s \geq 0 . \\
(a+b)^{p} \geq a^{p}+b^{p}+C a^{p-1} b, \quad \forall a, b \geq 0 .
\end{gathered}
$$

De fato, se considerarmos $t=\frac{b}{a}$ é suficiente mostrarmos que

$$
(1+t)^{p} \geq 1+t^{p}+C t, \quad \forall 0<t<1 .
$$

A desigualdade (A.31) segue do fato que

$$
\lim _{t \rightarrow 0^{+}} \frac{(1+t)^{p}-1-t^{p}}{t}=p>0,
$$

onde para determinarmos esse limite foi usado L'Hospital, agora da definição de limite temos que, para todo $\varepsilon>0$, existe um $\delta>0$, tal que $|t-0|<\delta$, implica que

$$
\left|\frac{(1+t)^{p}-1-t^{p}}{t}-p\right|<\varepsilon
$$

$\log 0$

$$
(1+t)^{p}>1+t^{p}+(p-\varepsilon) t .
$$

Assim, considerando a função $g(t)=(r+t)^{p}-r^{p}, t, r \geq 0$. Temos que

$$
\begin{aligned}
g(t) & =+(r+t)^{p}-r^{p}, \quad(r, t>0) \\
& \geq(r+t)^{p}-r^{p} \\
& \geq r^{p}+t^{p}+C a r^{p-1} t-r^{p} \\
& =t^{p}+C t r^{p-1}
\end{aligned}
$$

e

$$
\begin{aligned}
\int_{0}^{s} g_{\lambda}(t) d t & =\int_{0}^{s}(r+t)^{p}-r^{p} d t \\
& =\left(\frac{(r+t)^{p+1}}{p+1}-r^{p} t\right)_{0}^{s} \\
& =\frac{(r+s)^{p+1}}{p+1}-r^{p} s-\frac{r^{p+1}}{p+1} \\
& =\frac{(r+s)^{p+1}-r^{p+1}}{p+1}-r^{p} s
\end{aligned}
$$


Por outro lado,

$$
\begin{aligned}
\int_{0}^{s} g_{\lambda}(t) d t & =\int_{0}^{s}(r+t)^{p}-r^{p} d t \\
& \geq \int_{0}^{s} t^{p}-C r^{p-1} t d t \\
& =\left[\frac{t^{p+1}}{p+1-C r^{p-1} \frac{t^{2}}{2}}\right]_{0}^{s} \\
& =\frac{s^{p+1}}{p+1}-C r^{p-1} \frac{s^{2}}{2} .
\end{aligned}
$$

Obtendo assim

$$
\frac{(r+s)^{p+1}-r^{p+1}}{p+1}-r^{p} \geq \frac{s^{p+1}}{p+1}+C r^{p-1} s^{2}, \quad r, s \geq 0, p>1
$$

2. Mostraremos que para $r, s \geq 0,0<q<1$, que existe uma constante $C=C(q)>0$ tal que

$$
\frac{(r+s)^{q+1}-r^{q+1}}{q+1}-r^{q} s \leq C(q) s^{q+1}
$$

Poderíamos usar a desigualdade $(r+t)^{q}<r^{q}+t^{q}, r, t \geq 0,0<q<1$. Assim teríamos que

$$
\begin{aligned}
\int_{0}^{s}(r+t)^{q} d t & \leq \int_{0}^{s} r^{q}+t^{q} d t \\
\frac{(r+s)^{q+1}-r^{q+1}}{q+1} & \leq r^{q} s+\frac{s^{q+1}}{q+1} \\
\frac{(r+s)^{q+1}-r^{q+1}}{q+1}-r^{q} s & \leq \frac{s^{q+1}}{q+1} .
\end{aligned}
$$

Considerando $C=C(q)=\frac{1}{q+1}$, temos que, existe $C=C(q)$ tal que

$$
\frac{(r+s)^{q+1}-r^{q+1}}{q+1}-r^{q} s \leq C(q) s^{q+1}
$$

Lema A.6. $c_{\lambda}<\frac{S^{N / 2}}{N}$.

Demonstração. Seguindo a linha de demonstração de [13]. Defina

$$
v_{\varepsilon}(x):=\frac{C_{N} \varepsilon^{(N-2) / 2}}{\left(\varepsilon^{2}+|x|^{2}\right)^{(N-2) / 2}}
$$

onde $C_{N}=(N(N-2))^{(N-2) / 4}$, assim $v_{\varepsilon}$ satisfaz

$$
-\Delta v_{\varepsilon}=v_{\varepsilon}^{2^{*}-1}, \quad x \in \mathbb{R}^{N}
$$

Agora, escolha uma função $\eta \in C_{0}^{\infty}\left(B_{\rho}(0)\right)$ tal que $0 \leq \eta(x) \leq 1$ e $\eta(x)=1$ para todo $x \in B_{\rho / 2}(0), \rho>$ 0. E defina

$$
u_{\varepsilon}(x)=\eta(x) v_{\varepsilon}(x)
$$


para $\varepsilon_{0}$ suficientemente pequeno, existe $R>0$ tal que $J_{\lambda}\left(R u_{\varepsilon}\right)<0$, para todo $\varepsilon \in\left(0, \varepsilon_{0}\right)$.

Isto é, se escrevemos $\gamma(t)=t R u_{\varepsilon}, t \in[0,1], \operatorname{logo} \gamma \in \Gamma$. Assim

$$
c_{\lambda} \leq \max _{t \in[0,1]} J_{\lambda}\left(t u_{\varepsilon}\right)
$$

Assim precisamos mostrar que

$$
\max _{t \in[0,1]} J_{\lambda}\left(t u_{\varepsilon}\right)<\frac{S^{N / 2}}{N} .
$$

Primeiramente, note que

$$
\begin{aligned}
\left\|u_{\varepsilon}\right\|^{2} & =S^{N / 2}+O\left(\varepsilon^{N-2}\right) \\
\left\|u_{\varepsilon}\right\|_{2^{*}}^{2^{*}} & =S^{N / 2}+O\left(\varepsilon^{N}\right)
\end{aligned}
$$

e para algumas constantes $K_{1}, K_{2}$ e $K_{3}$ temos

$$
\left\|u_{\varepsilon}\right\|_{2}^{2}= \begin{cases}K_{1} \varepsilon^{2}+O\left(\varepsilon^{N-2}\right), & N \geq 5 \\ K_{2} \varepsilon^{2}\left|\ln \varepsilon^{2}\right|+O\left(\varepsilon^{2}\right), & N=4 \\ K_{3} \varepsilon+O\left(\varepsilon^{2}\right), & N=3\end{cases}
$$

Mais ainda

$$
\begin{aligned}
\int_{\Omega}\left|u_{\varepsilon}\right|^{q+1} d x & \leq \int_{B_{\varepsilon}} \frac{\left(C_{N} \varepsilon\right)^{\frac{(N-2)}{2}(q+1)}}{\varepsilon^{(N-2)(q+1)}} d x+\int_{B_{\rho} \backslash B_{\varepsilon}} \frac{\left(C_{N} \varepsilon\right)^{\frac{(N-2)}{2}(q+1)}}{|x|^{(N-2)(q+1)}} d x \\
& \leq C \varepsilon^{\frac{(N-2)(1-q)+4}{2}}+C \varepsilon^{\frac{(N-2)}{2}(q+1)} \int_{\varepsilon}^{\rho} r^{q(2-N)+1} d r
\end{aligned}
$$

e assim,

$$
\int_{\Omega}\left|u_{\varepsilon}\right|^{q+1} d x \leq \begin{cases}C \varepsilon^{\frac{(N-2)(1-q)+4}{2}}+C \varepsilon^{\frac{(N-2)}{2}(q+1)}, & q \neq \frac{2}{N-2} \\ C \varepsilon^{\frac{(N-2)(1-q)+4}{2}}+C \varepsilon^{\frac{(N-2)}{2}(q+1)}+C \varepsilon^{\frac{(N-2)}{2}(q+1)}|\ln \varepsilon|, & q=\frac{2}{N-2} .\end{cases}
$$

Então,

$$
\int_{\Omega}\left|u_{\varepsilon}\right|^{q+1} d x \leq\left\{\begin{array}{lr}
o_{n}\left(\varepsilon^{2}\right), & N \geq 6 \\
o_{n}\left(\varepsilon^{(N-2) / 2}\right), & 3 \leq N \leq 5 .
\end{array}\right.
$$

Agora, note que

$$
\begin{aligned}
\int_{B_{\rho}(0)} u_{\varepsilon}^{2^{*}} d x \leq \int_{B_{\rho}(o)}|x|^{\gamma} u_{\varepsilon}^{2^{*}} d x & =\int_{B_{\rho / \varepsilon}(0)}|\varepsilon x|^{\gamma} v_{\varepsilon}^{2^{*}}(\varepsilon x) d x+O\left(\varepsilon^{N}\right) \\
& =\varepsilon^{\gamma} \int_{B_{\rho / \varepsilon}(0)} \frac{|x|^{\gamma} C_{N}^{2^{*}}}{(1+|x|)^{N}} d x+O\left(\varepsilon^{N}\right) \\
& =O\left(\varepsilon^{\gamma}\right)+O\left(\varepsilon^{N}\right) .
\end{aligned}
$$

Agora dividimos a prova em dois casos.

1. Caso $N \geq 6$. 
Note que temos (Ver Lema anterior)

$$
\lambda\left[\frac{\left(t u_{\varepsilon}+u_{0}\right)^{q+1}-u_{0}^{q+1}}{q+1}-u_{0}^{q}\left(t u_{\varepsilon}\right)\right] \geq-C\left(t u_{\varepsilon}\right)^{q+1}
$$

e

$$
\left[\frac{\left(t u_{\varepsilon}+u_{0}^{p}\right)^{p+1}-u_{0}}{p+1}-u_{0}^{p}\left(t u_{\varepsilon}\right)\right] \geq\left[\frac{\left(t u_{\varepsilon}\right)^{p+1}}{p+1}+u_{0}^{p-1} \frac{\left(t u_{\varepsilon}\right)^{2}}{2}\right],
$$

mas lembre-se que $p=2^{*}-1$. Então

$$
\begin{aligned}
J_{\lambda}\left(t u_{\varepsilon}\right)= & \frac{1}{2} t^{2}\left\|u_{\varepsilon}\right\|^{2}-\int_{\Omega} G\left(t u_{\varepsilon}\right) d x \\
= & \frac{t^{2}}{2}\left\|u_{\varepsilon}\right\|^{2}-\int_{\Omega} \lambda\left[\frac{\left(t u_{\varepsilon}+u_{0}\right)^{q+1}-u_{0}^{q+1}}{q+1}-u_{0}^{q}\left(t u_{\varepsilon}\right)\right] d x \\
& +\int_{\Omega}\left[\frac{\left(t u_{\varepsilon}+u_{0}\right)^{p+1}-u_{0}^{p+1}}{p+1}-u_{0}^{p}\left(t u_{\varepsilon}\right)\right] d x .
\end{aligned}
$$

De $(A .41)$ e $(A .40)$ temos

$$
\begin{aligned}
J_{\lambda}\left(t u_{\varepsilon}\right) & \leq \frac{t^{2}}{2}\left\|u_{\varepsilon}\right\|^{2}+C \int_{\Omega}\left(t u_{\varepsilon}\right)^{q+1} d x-\int_{\Omega} \frac{\left(t u_{\varepsilon}\right)^{p+1}}{p+1} d x+\frac{u_{0}^{p-1}}{2} \\
& =\frac{t^{2}}{2}\left\|u_{\varepsilon}\right\|^{2}+C t^{q+1} \int_{\Omega} u_{\varepsilon}^{q+1} d x-\frac{t^{p+1}}{p+1} \int_{\Omega} u_{\varepsilon}^{p+1} d x-C \frac{t^{2}}{2} \int_{\Omega} u_{\varepsilon}^{2} d x \\
& =\frac{t^{2}}{2}\left(\left\|u_{\varepsilon}\right\|^{2}-C\left\|u_{\varepsilon}\right\|_{2}^{2}\right)-\frac{t^{2^{*}}}{2^{*}} \int_{\Omega} u_{\varepsilon}^{2^{*}} d x+C t^{q+1} \int_{\Omega} u_{\varepsilon}^{q+1} d x .
\end{aligned}
$$

Assim,

$$
J_{\lambda}\left(t u_{\varepsilon}\right) \leq \frac{1}{N}\left[\frac{\left\|u_{\varepsilon}\right\|^{2}-C\left\|u_{\varepsilon}\right\|_{2}^{2}}{\left(\int_{\Omega} u_{\varepsilon}^{2^{*}}\right)^{2 / 2^{*}}}\right]^{N / 2}+C t^{q+1} \int_{\Omega} u_{\varepsilon}^{q+1} d x
$$

Usando $(A .34),(A .35),(A .38)$ e $(A .39)$ temos

$$
\begin{aligned}
J_{\lambda}\left(t u_{\varepsilon}\right) & \leq \frac{1}{N}\left[\frac{S^{N / 2}-C \varepsilon^{2}+O\left(\varepsilon^{N-2}\right)}{\left(S^{N / 2}+O\left(\varepsilon^{\gamma}\right)+O\left(\varepsilon^{N}\right)\right)^{2 / 2^{*}}}\right]^{N / 2}+o_{n}\left(\varepsilon^{2}\right) \\
& =\frac{1}{N}\left[\frac{S^{N / 2}-C \varepsilon^{2}+o\left(\varepsilon^{2}\right)}{\left(S^{N / 2}+o\left(\varepsilon^{2}\right)\right)^{2 / 2^{*}}}\right]^{N / 2}+o_{n}\left(\varepsilon^{2}\right) \\
& =\frac{S^{N / 2}}{N}\left[1-C \varepsilon^{2}+o\left(\varepsilon^{2}\right)\right]+o_{n}\left(\varepsilon^{2}\right) \\
& <\frac{S^{N / 2}}{N}
\end{aligned}
$$

para $\varepsilon$ suficientemente pequeno (com $N \geq 6$, e $\gamma>2$ ).

2. Caso $3 \leq N \leq 5$ 
Usando (A.38) e a segunda desigualdade do Lema A.5, temos

$$
J_{\lambda}\left(t u_{\varepsilon}\right) \leq \frac{t^{2}}{2}\left\|u_{\varepsilon}\right\|^{2}-\frac{t^{2^{*}}}{2^{*}} \int_{\Omega} u_{\varepsilon}^{2^{*}} d x-C_{0} \frac{t^{p}}{p}\left\|u_{\varepsilon}\right\|_{p}^{p}+o_{n}\left(\varepsilon^{\frac{N-2}{2}}\right) .
$$

Note que $\left\|u_{\varepsilon}\right\|_{p}^{p}=C_{1} \varepsilon^{(N-2) / 2}+O\left(\varepsilon^{(N+2) / 2}\right), C_{1}>0$, segue que

$$
\begin{aligned}
J_{\lambda}\left(t u_{\varepsilon}\right) & \leq \frac{t^{2}}{2} S^{N / 2}-\frac{t^{2^{*}}}{2^{*}} S^{N / 2}-C \frac{t^{p}}{p} \varepsilon^{(N-2) / 2}+o\left(\varepsilon^{(N-2) / 2}\right)+O\left(\varepsilon^{(N+2) / 2}\right)+O\left(\varepsilon^{N}\right)+O\left(\varepsilon^{\gamma}\right) \\
& =\frac{t^{2}}{2} S^{N / 2}-\frac{t^{2^{*}}}{2^{*}} S^{N / 2}-C \frac{t^{p}}{p} \varepsilon^{(N-2) / 2}+o_{n}\left(\varepsilon^{(N-2) / 2}\right)
\end{aligned}
$$

usando $(A .34),(A .39)$ e que $\gamma>\frac{N-2}{2}$, chamando $t_{\varepsilon}$ o máximo do lado direito para $t \in[0,1]$, logo $t_{\varepsilon}$ satisfaz

$$
S^{N / 2}=t_{\varepsilon}^{2^{*}-2} S^{N / 2}+t_{\varepsilon}^{2^{*}-3} C \varepsilon^{(N-2) / 2}+o_{n}\left(\varepsilon^{(N-2) / 2}\right)
$$

assim

$$
t_{\varepsilon}=1-C \varepsilon^{(N-2) / 2} t_{\varepsilon}^{2^{*}-3}+o_{n}\left(\varepsilon^{(N-2) / 2}\right)
$$

Então

$$
\begin{aligned}
\max _{t \in[0, R]} J_{\lambda}\left(t u_{\varepsilon}\right) & \leq \frac{t_{\varepsilon}^{2}}{2} S^{N / 2}-\frac{t_{\varepsilon}^{2^{*}}}{2^{*}} S^{N / 2}-C t_{\varepsilon}^{2^{*}-3} \varepsilon^{(N-2) / 2}+o_{n}\left(\varepsilon^{(N-2) / 2}\right) \\
& =\frac{1}{2} S^{N / 2}-\frac{1}{2^{*}} S^{N / 2}-C t_{\varepsilon}^{2^{*}-3} \varepsilon^{(N-2) / 2}+o_{n}\left(\varepsilon^{(N-2) / 2}\right) \\
& =\frac{1}{N} S^{N / 2}-C t_{\varepsilon}^{2^{*}-3} \varepsilon^{(N-2) / 2}+o_{n}\left(\varepsilon^{(N-2) / 2}\right) \\
& <\frac{1}{N} S^{N / 2}
\end{aligned}
$$

para $\varepsilon$ suficientemente pequeno. Assim, $c_{\lambda}<\frac{1}{N} S^{N / 2}$ completando a prova do Lema.

Lema A.7. $c_{\lambda}<\frac{S^{N / 2}}{\|b\|_{\infty^{N-2}}^{\frac{N-2}{2}}}$.

Demonstração. A demonstração é análoga à demonstração do Lema A.6.

Defina

$$
v_{\varepsilon}(x):=\frac{C_{N} \varepsilon^{(N-2) / 2}}{\left(\varepsilon^{2}+|x|^{2}\right)^{(N-2) / 2}}
$$

onde $C_{N}=(N(N-2))^{(N-2) / 4}$, assim $v_{\varepsilon}$ satisfaz

$$
-\Delta v_{\varepsilon}=v_{\varepsilon}^{2^{*}-1}, \quad x \in \mathbb{R}^{N} .
$$

Agora, escolha uma função $\eta \in C_{0}^{\infty}\left(B_{\rho}(0)\right)$ tal que $0 \leq \eta(x) \leq 1$ e $\eta(x)=1$ para todo $x \in B_{\rho / 2}(0), \rho>$ 0. E defina

$$
u_{\varepsilon}(x)=\eta(x) v_{\varepsilon}(x),
$$

para $\varepsilon_{0}$ suficientemente pequeno, existe $R>0$ tal que $J_{\lambda}\left(R u_{\varepsilon}\right)<0$, para todo $\varepsilon \in\left(0, \varepsilon_{0}\right)$. 
Isto é, se escrevemos $\gamma(t)=t R u_{\varepsilon}, t \in[0,1], \operatorname{logo} \gamma \in \Gamma$. Assim

$$
c_{\lambda} \leq \max _{t \in[0,1]} J_{\lambda}\left(t u_{\varepsilon}\right)
$$

Assim precisamos mostrar que

$$
\max _{t \in[0,1]} J_{\lambda}\left(t u_{\varepsilon}\right)<\frac{S^{N / 2}}{\|b\|_{\infty^{2}}^{(N-2)}} N
$$

Primeiramente, note que

$$
\begin{aligned}
\left\|u_{\varepsilon}\right\|^{2} & =S^{N / 2}+O\left(\varepsilon^{N-2}\right) \\
\left\|u_{\varepsilon}\right\|_{2^{*}}^{2^{*}} & =S^{N / 2}+O\left(\varepsilon^{N}\right)
\end{aligned}
$$

e para algumas constantes $K_{1}, K_{2}$ e $K_{3}$ temos

$$
\left\|u_{\varepsilon}\right\|_{2}^{2}= \begin{cases}K_{1} \varepsilon^{2}+O\left(\varepsilon^{N-2}\right), & N \geq 5 \\ K_{2} \varepsilon^{2}\left|\ln \varepsilon^{2}\right|+O\left(\varepsilon^{2}\right), & N=4 \\ K_{3} \varepsilon+O\left(\varepsilon^{2}\right), & N=3\end{cases}
$$

Mais ainda

$$
\begin{aligned}
\int_{\Omega}\left|u_{\varepsilon}\right|^{q+1} d x & \leq \int_{B_{\varepsilon}} \frac{\left(C_{N} \varepsilon\right)^{\frac{(N-2)}{2}(q+1)}}{\varepsilon^{(N-2)(q+1)}} d x+\int_{B_{\rho} \backslash B_{\varepsilon}} \frac{\left(C_{N} \varepsilon\right)^{\frac{(N-2)}{2}(q+1)}}{|x|^{(N-2)(q+1)}} d x \\
& \leq C \varepsilon^{\frac{(N-2)(1-q)+4}{2}}+C \varepsilon^{\frac{(N-2)}{2}(q+1)} \int_{\varepsilon}^{\rho} r^{q(2-N)+1} d r
\end{aligned}
$$

e assim,

$$
\int_{\Omega}\left|u_{\varepsilon}\right|^{q+1} d x \leq \begin{cases}C \varepsilon^{\frac{(N-2)(1-q)+4}{2}}+C \varepsilon^{\frac{(N-2)}{2}(q+1)}, & q \neq \frac{2}{N-2} \\ C \varepsilon^{\frac{(N-2)(1-q)+4}{2}}+C \varepsilon^{\frac{(N-2)}{2}(q+1)}+C \varepsilon^{\frac{(N-2)}{2}(q+1)}|\ln \varepsilon|, & q=\frac{2}{N-2} .\end{cases}
$$

Então,

$$
\int_{\Omega}\left|u_{\varepsilon}\right|^{q+1} d x \leq\left\{\begin{array}{lr}
o_{n}\left(\varepsilon^{2}\right), & N \geq 6 \\
o_{n}\left(\varepsilon^{(N-2) / 2}\right), & 3 \leq N \leq 5
\end{array}\right.
$$

Agora, note que

$$
\int_{B_{\rho}(0)} b(x) u_{\varepsilon}^{2^{*}} d x=\|b\|_{\infty} \int_{B_{\rho}(0)} u_{\varepsilon}^{2^{*}} d x-\int_{B_{\rho}(0)}\left(\|b\|_{\infty}-b(x)\right) u_{\varepsilon}^{2^{*}} d x
$$

Assim, utilizando a hipótese (b) e fazendo uma mudança de variáveis, temos

$$
\begin{aligned}
\int_{B_{\rho}(0)}\left(\|b\|_{\infty}-b(x)\right) u_{\varepsilon}^{2^{*}} d x \leq \int_{B_{\rho}(o)}|x|^{\gamma} u_{\varepsilon}^{2^{*}} d x & =\int_{B_{\rho / \varepsilon}(0)}|\varepsilon x|^{\gamma} v_{\varepsilon}^{2^{*}}(\varepsilon x) d x+O\left(\varepsilon^{N}\right) \\
& =\varepsilon^{\gamma} \int_{B_{\rho / \varepsilon}(0)} \frac{|x|^{\gamma} C_{N}^{2^{*}}}{(1+|x|)^{N}} d x+O\left(\varepsilon^{N}\right) \\
& =O\left(\varepsilon^{\gamma}\right)+O\left(\varepsilon^{N}\right) .
\end{aligned}
$$


Então

$$
\int_{B_{\rho}(0)} b(x) u_{\varepsilon}^{2^{*}}=\|b\|_{\infty} u_{\varepsilon}^{2^{*}} d x=\|b\|_{\infty}\left\|u_{\varepsilon}\right\|_{2^{*}}^{2^{*}}+O\left(\varepsilon^{\gamma}\right)+O\left(\varepsilon^{N}\right) .
$$

Agora dividimos a prova em dois casos.

1. Caso $N \geq 6$.

Note que temos (Ver Lema anterior)

$$
a(x)\left[\frac{\left(t u_{\varepsilon}+u_{0}\right)^{q+1}-u_{0}^{q+1}}{q+1}-u_{0}^{q}\left(t u_{\varepsilon}\right)\right] \geq-C\left(t u_{\varepsilon}\right)^{q+1}
$$

e

$$
b(x)\left[\frac{\left(t u_{\varepsilon}+u_{0}^{p}\right)^{p+1}-u_{0}}{p+1}-u_{0}^{p}\left(t u_{\varepsilon}\right)\right] \geq b(x)\left[\frac{\left(t u_{\varepsilon}\right)^{p+1}}{p+1}+u_{0}^{p-1} \frac{\left(t u_{\varepsilon}\right)^{2}}{2}\right],
$$

mas lembre-se que $p=2^{*}-1$. Então

$$
\begin{aligned}
J_{\lambda}\left(t u_{\varepsilon}\right)= & \frac{1}{2} t^{2}\left\|u_{\varepsilon}\right\|^{2}-\int_{\Omega} G\left(t u_{\varepsilon}\right) d x \\
= & \frac{t^{2}}{2}\left\|u_{\varepsilon}\right\|^{2}-\int_{\Omega} a(x) \lambda\left[\frac{\left(t u_{\varepsilon}+u_{0}\right)^{q+1}-u_{0}^{q+1}}{q+1}-u_{0}^{q}\left(t u_{\varepsilon}\right)\right] d x \\
& +\int_{\Omega} b(x)\left[\frac{\left(t u_{\varepsilon}+u_{0}\right)^{p+1}-u_{0}^{p+1}}{p+1}-u_{0}^{p}\left(t u_{\varepsilon}\right)\right] d x
\end{aligned}
$$

De $(A .55)$ e $(A .54)$ temos

$$
\begin{aligned}
J_{\lambda}\left(t u_{\varepsilon}\right) & \leq \frac{t^{2}}{2}\left\|u_{\varepsilon}\right\|^{2}+C \int_{\Omega}\left(t u_{\varepsilon}\right)^{q+1} d x-\int_{\Omega} b(x) \frac{\left(t u_{\varepsilon}\right)^{p+1}}{p+1} d x+\frac{u_{0}^{p-1}}{2} \\
& =\frac{t^{2}}{2}\left\|u_{\varepsilon}\right\|^{2}+C t^{q+1} \int_{\Omega} u_{\varepsilon}^{q+1} d x-\frac{t^{p+1}}{p+1} \int_{\Omega} b(x) u_{\varepsilon}^{p+1}-C \frac{t^{2}}{2} \int_{\Omega} u_{\varepsilon}^{2} d x \\
& =\frac{t^{2}}{2}\left(\left\|u_{\varepsilon}\right\|^{2}-C\left\|u_{\varepsilon}\right\|_{2}^{2}\right)-\frac{t^{2^{*}}}{2^{*}} \int_{\Omega} b(x) u_{\varepsilon}^{2^{*}}+C t^{q+1} \int_{\Omega} u_{\varepsilon}^{q+1} d x
\end{aligned}
$$

Assim,

$$
J_{\lambda}\left(t u_{\varepsilon}\right) \leq \frac{1}{N}\left[\frac{\left\|u_{\varepsilon}\right\|^{2}-C\left\|u_{\varepsilon}\right\|_{2}^{2}}{\left(\int_{\Omega} b(x) u_{\varepsilon}^{2^{*}}\right)^{2 / 2^{*}} d x}\right]^{N / 2}+C t^{q+1} \int_{\Omega} u_{\varepsilon}^{q+1} d x
$$


Usando $(A .48),(A .49),(A .52)$ e $(A .53)$ temos

$$
\begin{aligned}
J_{\lambda}\left(t u_{\varepsilon}\right) & \leq \frac{1}{N}\left[\frac{S^{N / 2}-C \varepsilon^{2}+O\left(\varepsilon^{N-2}\right)}{\left(\|b\|_{\infty} S^{N / 2}+O\left(\varepsilon^{\gamma}\right)+O\left(\varepsilon^{N}\right)\right)^{2 / 2^{*}}}\right]^{N / 2}+o_{n}\left(\varepsilon^{2}\right) \\
& =\frac{1}{N}\left[\frac{S^{N / 2}-C \varepsilon^{2}+o\left(\varepsilon^{2}\right)}{\left(\|b\|_{\infty} S^{N / 2}+o\left(\varepsilon^{2}\right)\right)^{2 / 2^{*}}}\right]^{N / 2}+o_{n}\left(\varepsilon^{2}\right) \\
& =\frac{S^{N / 2}}{\|b\|_{\infty^{\frac{N-2}{2}}} N}\left[1-C \varepsilon^{2}+o\left(\varepsilon^{2}\right)\right]+o_{n}\left(\varepsilon^{2}\right) \\
& <\frac{S^{N / 2}}{\|b\|_{\infty^{\frac{N-2}{2}}}^{2} N}
\end{aligned}
$$

para $\varepsilon$ suficientemente pequeno $(\operatorname{com} N \geq 6$, e $\gamma>2)$.

2. Caso $3 \leq N \leq 5$

(A.38) e a segunda desigualdade do Lema A.5, temos

$$
J_{\lambda}\left(t u_{\varepsilon}\right) \leq \frac{t^{2}}{2}\left\|u_{\varepsilon}\right\|^{2}-\frac{t^{2^{*}}}{2^{*}} \int_{\Omega} b(x) u_{\varepsilon}^{2^{*}} d x-C_{0} \frac{t^{p}}{p}\left\|u_{\varepsilon}\right\|_{p}^{p}+o_{n}\left(\varepsilon^{\frac{N-2}{2}}\right), C_{0}>0
$$

Note que $\left\|u_{\varepsilon}\right\|_{p}^{p}=C_{1} \varepsilon^{(N-2) / 2}+O\left(\varepsilon^{(N+2) / 2}\right), C_{1}>0$, segue que

$$
\begin{aligned}
J_{\lambda}\left(t u_{\varepsilon}\right) & \leq \frac{t^{2}}{2} S^{N / 2}-\frac{t^{2^{*}}}{2^{*}}\|b\|_{\infty} S^{N / 2}-C \frac{t^{p}}{p} \varepsilon^{(N-2) / 2}+o\left(\varepsilon^{(N-2) / 2}\right)+O\left(\varepsilon^{(N+2) / 2}\right)+O\left(\varepsilon^{N}\right)+O\left(\varepsilon^{\gamma}\right) \\
& =\frac{t^{2}}{2}\|b\|_{\infty} S^{N / 2}-\frac{t^{2^{*}}}{2^{*}} S^{N / 2}-C \frac{t^{p}}{p} \varepsilon^{(N-2) / 2}+o_{n}\left(\varepsilon^{(N-2) / 2}\right)
\end{aligned}
$$

usando $(A .48),(A .53)$ e que $\gamma>\frac{N-2}{2}$, chamando $t_{\varepsilon}$ o máximo do lado direito para $t \in[0,1], \operatorname{logo}$ $t_{\varepsilon}$ satisfaz

$$
S^{N / 2}=t_{\varepsilon}^{2^{*}-2}\|b\|_{\infty} S^{N / 2}+t_{\varepsilon}^{2^{*}-3} C \varepsilon^{(N-2) / 2}+o_{n}\left(\varepsilon^{(N-2) / 2}\right)
$$

assim

$$
t_{\varepsilon}=\frac{1}{\|b\|_{\infty}^{\frac{N-2}{\infty^{4}}}}-C \varepsilon^{(N-2) / 2} t_{\varepsilon}^{2^{*}-3}+o_{n}\left(\varepsilon^{(N-2) / 2}\right) .
$$

Então

$$
\begin{aligned}
\max _{t \in[0, R]} J_{\lambda}\left(t u_{\varepsilon}\right) & \leq \frac{t_{\varepsilon}^{2}}{2} S^{N / 2}-\frac{t_{\varepsilon}^{2^{*}}}{2^{*}}\|b\|_{\infty} S^{N / 2}-C t_{\varepsilon}^{2^{*}-3} \varepsilon^{(N-2) / 2}+o_{n}\left(\varepsilon^{(N-2) / 2}\right) \\
& =\frac{1}{2} \frac{S^{N / 2}}{\|b\|_{\infty}^{\frac{N-2}{2}}}-\frac{1}{2^{*}} \frac{S^{N / 2}}{\|b\|_{\infty}^{\frac{N-2}{2}}}-C t_{\varepsilon}^{2^{*}-3} \varepsilon^{(N-2) / 2}+o_{n}\left(\varepsilon^{(N-2) / 2}\right) \\
& =\frac{1}{N} \frac{S^{N / 2}}{\|b\|_{\infty^{\frac{N-2}{2}}}}-C t_{\varepsilon}^{2^{*}-3} \varepsilon^{(N-2) / 2}+o_{n}\left(\varepsilon^{(N-2) / 2}\right) \\
& <\frac{1}{N} \frac{S^{N / 2}}{\|b\|_{\infty}^{\frac{N-2}{2}}},
\end{aligned}
$$

para $\varepsilon$ suficientemente pequeno. Assim, $c_{\lambda}<\frac{1}{N} S^{N / 2}$ completando a prova do Lema. 
Considerando o seguinte problema

$$
(P) \quad\left\{\begin{aligned}
-\Delta u=f(x, u), & x \in \Omega, \\
u \geq 0, u \neq 0, & x \in \Omega, \\
u=0, & x \in \partial \Omega .
\end{aligned}\right.
$$

Onde, $\Omega$ é um domínio limitado de $\mathbb{R}^{N}$ e $f: \Omega \times \mathbb{R}^{+} \rightarrow \mathbb{R}$ é uma função de Carathéodory.

Definição A.8 (Sublinearidade). Dizemos que o Problema $(P)$ é sublinear no 0 se existe $\alpha>\lambda_{1}(\Omega) e$ $s_{0}>0$ tal que

$$
f(x, s) \geq \alpha s, \text { para quase todo ponto em } \Omega \text { e todo } 0 \leq s \leq s_{0}
$$

Onde $\lambda_{1}(\Omega)$ denota o primeiro autovalor de $-\Delta$ sobre $H$.

Definição A.9 (Superlinearidade). Dizemos que o Problema $(P)$ é superlinear no $\infty$ se existe $\beta>\lambda_{1}(\Omega)$ e $s_{1} \geq 0$ tal que

$$
f(x, s) \geq \beta s, \text { para quase todo ponto em } \Omega \text { e todo } s \geq s_{1} \text {. }
$$

Proposição A.2. Sejam $\Omega$ um dominio limitado e $f: \Omega \times \mathbb{R} \rightarrow \mathbb{R}$ uma função de Carathéodory. Suponha que existam $1 \leq \sigma<2^{*}-1, d_{1} \in L^{\sigma^{\prime}}(\Omega)$ e $d_{2}>0$ tais que

$$
|f(x, s)| \leq d_{1}(x)+d_{2}|s|^{\sigma-1}
$$

em quase todo ponto de $\Omega$ e para todo $s \in \mathbb{R}$. Ent ao o funcional $J$ definido como $J(u)=\int_{\Omega} F(x, u) d x$ com $F(x, u)=\int_{0}^{u} f_{\lambda}(x, t) d t$ é de classe $C^{1}$ de $H$ em $\mathbb{R}$, com

$$
J^{\prime}(u) v=\int_{\Omega} f(x, u) v d x
$$

para todo $v \in H$. Além disso,

$$
J^{\prime}: H \rightarrow H^{-1}
$$

é compacto.

\section{A.4 Funcionais com simetria e teoria de índice}

As aplicações mais notáveis dos métodos de minimax são resultados que garantem a existência de múltiples pontos críticos de funcionais os quais são invariante sob a ação de um grupo de simetrias. Nesta seção apresentaremos algumas ferramentas para tratar o estudo da multiplicidade de soluções. Para isso, seja $E$ um espaço de Banach real, $\mathcal{G}$ um grupo de aplicaøões de $E$ sobre $E$, e $I \in C^{1}(E, \mathbb{R})$. Dizemos que $I$ é invariante por $\mathcal{G}$ se $I(g u)=I(u)$ para tudo $g \in \mathcal{G}$ e $u \in E$. Como exemplo, suponha que $I$ é par, isto é, $I(u)=I(-u)$ para tudo $u \in E$. Logo $I$ é invariante por $\mathcal{G}=\{i d,-i d\} \simeq \mathbb{Z}_{2}$.

Definição A.4.1. (Ver [21]) Seja E um espaço de Banach real e seja $\mathcal{E}$ que denota a família de conjuntos $A \subset E \backslash\{0\}$ tal que $A$ é fechado em $E$ e é simétrica com respeito a 0 , isto é, $x \in A$ implica que $-x \in A$. Para $A \in \mathcal{E}$, definimos o gênero de $A$, como sendo $n$ (denotado por $\gamma(A)=n$ ) se existe uma aplicação 
par $\varphi \in C\left(A, \mathbb{R}^{n} \backslash\{0\}\right)$ e n é o inteiro mais pequeno que satisfaz esta propriedade. Se não existir tal $n$ finito, dizemos que $\gamma(A)=\infty$. Além disso, definimos que $\gamma(\emptyset)=0$.

Observação A.1. Se $A \in \mathcal{E}$ e $\gamma(A)>1$, logo A contém infinitos pontos distintos.

As principais propriedades do gênero são coletados na seguinte proposição. Para $A \in \mathcal{E}$ e $\delta>0$, seja $N_{\delta}(A)$ que denota uma $\delta$-vizinhança uniforme de $A$, isto é, $N_{\delta}(A)=\{x \in E \mid\|x-A\| \leq \delta\}$.

Proposição A.3. (Ver [21]) Seja $A, B \in \mathcal{E}$. Logo,

1. Se $x \neq 0, \gamma(\{x\} \cup\{-x\})=1$;

2. Se existe uma aplicação par $f \in C(A, B) \operatorname{logo} \gamma(A) \leq \gamma(B)$;

3. Se $A \subset B, \gamma(A) \leq \gamma(B)$;

4. Se A é compacto, $\gamma(A)<\infty$ e existe um $\delta>0$ é tal que $N_{\delta}(A) \in \mathcal{E}$ e $\gamma\left(N_{\delta}(A)\right)=\gamma(A)$.

No seguinte resultado, calculamos o gênero de uma importante classe de conjuntos.

Proposição A.4. (Ver [21]) Se $A \subset \mathcal{E}, \Omega$ é uma vizinhança simétrica e limitada de 0 em $\mathbb{R}^{k}$, e existe uma aplicação $h \in C(A, \partial \Omega)$ com $h$ um homeomorfismo impar, logo $\gamma(A)=k$.

Corolário A.1. $\gamma\left(S^{m-1}\right)=m$.

Teorema A.15. (Ver[21]) Se $I \in C^{1}(E, \mathbb{R})$ funcional par, logo $\left.I\right|_{S^{m-1}}$ tem ao menos $m$ pares de pontos críticos distintos. 


\section{Apêndice}

$\mathcal{B}$

\section{Teorema do Passo da Montanha}

\section{B.1 Lema de Deformação Quantitativo}

O lema da deformação é um resultado que garante a existência de uma aplicação $\eta \in C([0,1] \times X, X)$ tal que, dado $\varepsilon>0$ pequeno, podemos deformar o conjunto $I^{c+\varepsilon}$ no conjunto $I^{c-\varepsilon}$, onde aqui $c$ é um valor regular de $I$, isto é, não existe pontos críticos do funcional $I$ no nível $c$. No sentido topológico, os conjuntos $I^{c+\epsilon}$ e $I^{c-\epsilon}$ são iguais. O lema de deformação que aqui apresentaremos é devido a Willem [25]. Para um melhor entendimento, fixaremos uma notação, isto é, iremos denotar por $X$ um espaço de Banach com norma $\|\cdot\|$, denotaremos também por $\|\cdot\|_{X^{\prime}}$ a norma no dual de $X$ e por $|\cdot|_{p}$ a norma em $L^{p}(\Omega)$ e para todo número real $d$ e $I: X \rightarrow \mathbb{R}$, definimos $I^{d}=\{u \in X: I(u) \leq d\}$. Para o estudo do Lema de Deformação Quantitativo vamos a precisar do seguinte resultado.

\section{B.1.1 Campo Pseudo-Gradiente}

Seja $X$ um espaço de Banach e $I \in C^{1}(X, \mathbb{R})$. Dizemos que $v \in X$ é um vetor pseudo-gradiente para $I$ em $u \in \tilde{X}=\left\{w \in X: I^{\prime}(w) \neq 0\right\}$ se

$$
\|v\| \leq 2\left\|I^{\prime}(u)\right\|_{X^{\prime}} \quad \text { e } \quad I^{\prime}(u) v \geq\left\|I^{\prime}(u)\right\|_{X^{\prime}}^{2} .
$$

Um campo pseudo-gradiente para $I$ em $X$ é uma aplicação $V: \tilde{X} \rightarrow X$ tal que

(a) $V$ é localmente Lipschitziana;

(b) para cada $u \in \tilde{X}, V(u)$ é um vetor pseudo-gradiente para $I$.

Lema B.1. Se $I \in C^{1}(X, \mathbb{R})$ então existe um campo pseudo-gradiente para I em $\tilde{X}$.

Demonstração. Dado $u \in \tilde{X}$ existe, por definição, $w \in X$ tal que $\|w\|_{X}=1 \mathrm{e}$

$$
I^{\prime}(u) w>\frac{2}{3}\left\|I^{\prime}(u)\right\|_{X^{\prime}} .
$$


Então $z=\frac{3}{2}\left\|I^{\prime}(u)\right\| w$ é um vetor pseudo-gradiente para $I$ em $u$. De fato,

$$
\|z\|=\frac{3}{2}\left\|I^{\prime}(u)\right\|_{X^{\prime}}\|w\|=\frac{3}{2}\left\|I^{\prime}(u)\right\|_{X^{\prime}}<2\left\|I^{\prime}(u)\right\|_{X^{\prime}}
$$

$$
I^{\prime}(u) z=\frac{3}{2}\left\|I^{\prime}(u)\right\|_{X^{\prime}} I^{\prime}(u) w>\frac{3}{2}\left\|I^{\prime}(u)\right\|_{X^{\prime}} \frac{2}{3}\left\|I^{\prime}(u)\right\|_{X^{\prime}}=\left\|I^{\prime}(u)\right\|_{X^{\prime}}^{2} .
$$

Pela continuidade de $I^{\prime}$, existe uma vizinhança $N_{u}$ de $u$ tal que para todo $v \in N_{u}$

$$
I^{\prime}(v) z \geq\left\|I^{\prime}(v)\right\|_{X^{\prime}}^{2}, \quad \text { e } \quad\|z\| \leq 2\left\|I^{\prime}(v)\right\|_{X^{\prime}} .
$$

Note que a família $\mathcal{N}=\left\{N_{u}\right\}_{u \in \tilde{X}}$ é uma cobertura aberta de $\tilde{X}$. Como $\tilde{X}$ é um espaço métrico, então é paracompacto (ver Apêndice $A$, definição A.1). Logo, existe uma cobertura $\mathcal{M}=\left\{M_{\lambda}\right\}_{\lambda \in \Lambda}$ de $\tilde{X}$, aberta e localmente finita que refina $\mathcal{N}$, isto é, para cada $u \in \tilde{X}$ existem índices $\lambda_{1}, \ldots, \lambda_{n} \in \Lambda$ e uma vizinhança $W_{u}$ de $u$ tal que $W_{u} \cap M_{\lambda} \neq \emptyset \operatorname{com} \lambda \in \Lambda_{n}=\left\{\lambda_{1}, \ldots, \lambda_{n}\right\}$, ou seja, a intersecção citada é não vazia apenas para um número finito de índices $\lambda$. Além disso, para cada $M_{\lambda} \in \mathcal{M}$ existe $N_{u} \in \mathcal{N}$ tal que $M_{\lambda} \subset N_{u}$. Seja $z_{\lambda}$ um vetor pseudo-gradiente para $I$ em $M_{\lambda}$. Então $z_{\lambda}=z$ satisfaz (B.1) para cada $u \in M_{\lambda}$.

Seja agora $d_{\lambda}(u)$ a distância de $u$ ao complemento de $M_{\lambda}$. Então a função $d_{\lambda}$ é Lipschitziana e além disso $\operatorname{supp}\left(d_{\lambda}\right) \subseteq M_{\lambda}$. Defina

$$
f_{\lambda}(u)=\frac{d_{\lambda} u}{\sum_{k \in \Lambda_{n}} d_{k}(u)} .
$$

Observe que $f_{\lambda}$ está bem definida, pois para cada $u \in \tilde{X}, \sum_{k \in \Lambda_{n}} d_{k}(u)<\infty$ é não nulo. Além disso como o refinamento é localmente finito, tem-se que, $0 \leq f_{\lambda} \leq 1$ e para cada $u \in \tilde{X}$,

$$
\sum_{\lambda \in \Lambda} f_{\lambda}(u)=\sum_{\lambda \in \Lambda} \frac{d_{\lambda}(u)}{\sum_{k \in \Lambda_{n}} d_{k}(u)}=1 .
$$

Verifiquemos agora que

$$
V(u):=\sum_{\lambda \in \Lambda} f_{\lambda}(u) z_{\lambda}
$$

é um campo pseudo-gradiente para $I$ em $\tilde{X}$. Temos por (B.1) e (B.2) que

$$
\|V(u)\| \leq \sum_{\lambda \in \Lambda}\left|d_{\lambda}(u) / \sum_{k \in \Lambda_{n}} d_{k}(u)\right|\|z\|<2\left\|I^{\prime}(u)\right\|_{X^{\prime}},
$$

e novamente de (B.1) segue que

$$
\begin{aligned}
I^{\prime}(u) V(u) & =I^{\prime}(u) \sum_{\lambda \in \Lambda} f_{\lambda}(u) z_{\lambda} \\
& =\frac{3}{2} \sum_{\lambda \in \Lambda} f_{\lambda}(u)\left\|I^{\prime}(u)\right\|_{X^{\prime}} I^{\prime}(u) w \\
& \geq\left\|I^{\prime}(u)\right\|_{X^{\prime}}^{2},
\end{aligned}
$$

ou seja

$$
I^{\prime}(u) V(u) \geq\left\|I^{\prime}(u)\right\|_{X^{\prime}}^{2} .
$$


Portanto, segue de (B.3) e (B.4) que $V$ é um campo pseudo-gradiente para $I$ em $\tilde{X}$. Resta mostrar que $V$ é localmente Lipschitziana. Para isso, basta observar que $V$ é uma soma finita de funções localmente Lipchitzianas, visto que a cobertura $\mathcal{M}$ de $\tilde{X}$ é um refinamento localmente finito de $\mathcal{N}$ e as funções $d_{\lambda}$ são localmente Lipschitzianas. Portanto, $V$ é um campo pseudo-gradiente para $I$ em $\tilde{X}$.

Lema B.2. (Lema de Deformação Quantitativo) Sejam $X$ um espaço de Banach, $S \subseteq X, \delta>0$ e defina

$$
S_{\delta}=\{u \in X: \operatorname{dist}(u, S) \leq \delta\} .
$$

Sejam $I \in C^{1}(X, \mathbb{R}), c \in \mathbb{R} e \varepsilon>0$ tais que

$$
\left\|I^{\prime}(u)\right\|_{X^{\prime}} \geq \frac{4 \varepsilon}{\delta}, \forall u \in I^{-1}([c-2 \varepsilon, c+2 \varepsilon]) \cap S_{2 \delta} .
$$

Então existe uma função $\eta \in C([0,1] \times X, X)$ de tal forma que:

1. $\quad \eta(0, u)=u, \forall u \in X$;

2. $\eta(t, u)=u, \forall(t, u) \notin[0,1] \times I^{-1}([c-2 \varepsilon, c+2 \varepsilon])$;

3. $\eta\left(1, I^{c+\varepsilon} \cap S\right) \subseteq I^{c-\varepsilon} \cap S_{\delta}$.

Demonstração. Seja $\tilde{X}=\left\{u \in X: I^{\prime}(u) \neq 0\right\}$. Pelo Lema B.1 temos que existe um campo pseudogradiente para $I$ em $\tilde{X}$, isto é, uma aplicação

$$
v: \tilde{X} \rightarrow X
$$

tal que, para todo $u \in \tilde{X}$,

(D1) $\|v(u)\|_{X} \leq 2\left\|I^{\prime}(u)\right\|_{X^{\prime}}$;

(D2) $I^{\prime}(u) v(u) \geq\left\|I^{\prime}(u)\right\|_{X^{\prime}}^{2}$.

Defina

$$
A=I^{-1}([c-2 \varepsilon, c+2 \varepsilon]) \cap S_{2 \delta} \quad \text { e } \quad B=I^{-1}([c-\varepsilon, c+\varepsilon]) \cap S_{2 \delta}
$$

e $\psi: X \rightarrow \mathbb{R}$ dada por

$$
\psi(u)=\frac{d(u, X \backslash A)}{d(u, X \backslash A)+d(u, B)}
$$

Note que

$$
0 \leq \psi \leq 1, \psi \equiv 0 \text { em } X \backslash A \text { e } \psi \equiv 1 \text { em } B .
$$

Mostraremos que $\psi$ é localmente Lipschitziana. De fato, considere $u_{1}, u_{2} \in X$ e denotaremos, para $i=1,2$,

$$
d_{u, X, A}^{i}=d\left(u_{i}, X \backslash A\right) \text { e } d_{u, B}^{i}=d\left(u_{i}, B\right)
$$


Logo

$$
\begin{aligned}
\mid \psi\left(u_{1}\right)-\psi\left(u_{2}\right) & =\left|\frac{\left(d_{u, X, A}^{2}+d_{u, B}^{2}\right) d_{u, X, A}^{1}-\left(d_{u, X, A}^{1}+d_{u, B}^{1}\right) d_{u, X, A}^{2}}{\left(d_{u, X, A}^{1}+d_{u, B}^{1}\right)\left(d_{u, X, A}^{2}+d_{u, B}^{2}\right)}\right| \\
& =\left|\frac{d_{u, X, A}^{1} d_{u, B}^{2}-d_{u, X, A}^{2} d_{u, B}^{1}}{\left(d_{u, X, A}^{1}+d_{u, B}^{1}\right)\left(d_{u, X, A}^{2}+d_{u, B}^{2}\right)}\right| \\
& =\left|\frac{d_{u, X, A}^{1} d_{u, B}^{2}-d_{u, X, A}^{2} d_{u, B}^{2}+d_{u, X, A}^{2} d_{u, B}^{2}-d_{u, X, A}^{2} d_{u, B}^{1}}{\left(d_{u, X, A}^{1}+d_{u, B}^{1}\right)\left(d_{u, X, A}^{2}+d_{u, B}^{2}\right)}\right| \\
& =\left|\frac{d_{u, B}^{2}\left(d_{u, X, A}^{1}-d_{u, X, A}^{2}\right)+d_{u, X, A}^{2}\left(d_{u, B}^{2}-d_{u, B}^{1}\right)}{\left(d_{u, X, A}^{1}+d_{u, B}^{1}\right)\left(d_{u, X, A}^{2}+d_{u, B}^{2}\right)}\right| .
\end{aligned}
$$

Desde que a função distância é uma contração fraca, veja [14][Ex.3, pag.31] temos que

$$
\left|d_{u, X, A}^{1}-d_{u, X, A}^{2}\right| \leq\left\|u_{1}-u_{2}\right\| \quad \text { e } \quad\left|d_{u, B}^{2}-d_{u, B}^{1}\right| \leq\left\|u_{1}-u_{2}\right\|
$$

Logo,

$$
\left|\psi\left(u_{1}\right)-\psi\left(u_{2}\right)\right| \leq \frac{\left\|u_{1}-u_{2}\right\| d_{u, B}^{2}+\left\|u_{1}-u_{2}\right\| d_{u, X, A}^{2}}{\left(d_{u, X, A}^{1}+d_{u, B}^{1}\right)\left(d_{u, X, A}^{2}+d_{u, B}^{2}\right)}=\frac{\left\|u_{1}-u_{2}\right\|}{d_{u, X, A}^{1}+d_{u, B}^{1}} .
$$

Utilizando o fato de que a função distância é localmente Lipschitziana. Temos que, para qualquer que seja $w \in X$,

$$
d_{w, X, A}^{1}+d_{w, B}^{1}>0
$$

Logo, existe uma constante $k>0$ e uma vizinhança $W$ de $w$ tal que

$$
d \frac{1}{w, X, A}+d_{\bar{w}, B}^{1} \geq \frac{1}{k}>0 \text { para todo } \bar{w} \in W .
$$

Assim,

$$
\left|\psi\left(u_{1}\right)-\psi\left(u_{2}\right)\right| \leq k\left\|u_{1}-u_{2}\right\|
$$

dessa forma $\psi$ é localmente Lipschitziana.

Definimos agora a função $\Phi: X \rightarrow X$ por

$$
\Phi(u)=\left\{\begin{array}{cl}
-\psi(u) \frac{v(u)}{\|v(u)\|^{2}}, & u \in A, \\
0, & u \in \overline{X \backslash A} .
\end{array}\right.
$$

Por $(D 2)$ e por $B .1$ temos

$$
\|\Phi(u)\| \leq \frac{1}{\|v(u)\|} \leq \frac{1}{\left\|I^{\prime}(u)\right\|} \leq \frac{\delta}{4 \varepsilon} .
$$

Note que, dado $u \in X$ existe uma vizinhança $B_{u}$ tal que $\psi$ e $v$ são localmente Lipschitzianas em $B_{u}$. Agora considere $u_{1}, u_{2} \in B_{u}$ e sejam 


$$
f\left(u_{i}\right)=\frac{v\left(u_{i}\right)}{\left\|v\left(u_{i}\right)\right\|^{2}} \text { e } f_{i}\left(u_{j}\right)=\frac{v\left(u_{i}\right)}{\left\|v\left(u_{j}\right)\right\|^{2}} \text { para } i, j=1,2 .
$$

Logo, se $u_{1}, u_{2} \in \overline{X \backslash A}$, temos,

$$
\left\|\Phi\left(u_{1}\right)-\Phi\left(u_{2}\right)\right\|=0 \leq\left\|u_{1}-u_{2}\right\| .
$$

Se considerarmos $u_{1} \in A$ e $u_{2} \in \overline{X \backslash A}$, obtemos que,

$$
\begin{aligned}
\left\|\Phi\left(u_{1}\right)-\Phi\left(u_{2}\right)\right\| & =\left\|-\psi\left(u_{1}\right) f\left(u_{1}\right)\right\| \\
& =\left\|-\psi\left(u_{1}\right) f\left(u_{1}\right)+\psi\left(u_{2}\right) f\left(u_{1}\right)\right\| \\
& \leq \frac{\delta}{4 \varepsilon}\left|\psi\left(u_{1}\right)-\psi\left(u_{2}\right)\right| \quad \text { por } B .7 \\
& \leq \frac{k \delta}{4 \varepsilon}\left\|u_{1}-u_{2}\right\| \quad \text { por } B .6 \\
& =k_{1}\left\|u_{1}-u_{2}\right\| .
\end{aligned}
$$

Assim, se $u_{1}, u_{2} \in A$, então

$$
\begin{aligned}
\left\|\Phi\left(u_{1}\right)-\Phi\left(u_{2}\right)\right\| & =\left\|-\psi\left(u_{1}\right) f\left(u_{1}\right)+\psi\left(u_{2}\right) f\left(u_{2}\right)\right\| \\
& =\left\|-\psi\left(u_{1}\right) f\left(u_{1}\right)+\psi\left(u_{1}\right) f\left(u_{2}\right)-\psi\left(u_{1}\right) f\left(u_{2}\right)+\psi\left(u_{2}\right) f\left(u_{2}\right)\right\| \\
& \leq\left\|f\left(u_{1}\right)-f\left(u_{2}\right)\right\|+\frac{\delta}{4 \varepsilon}\left|\psi\left(u_{1}\right)-\psi\left(u_{2}\right)\right| \\
& \leq\left\|f\left(u_{1}\right)-f_{1}\left(u_{2}\right)\right\|+\left\|f_{1}\left(u_{2}\right)-f\left(u_{2}\right)\right\|+\frac{\delta}{4 \varepsilon}\left|\psi\left(u_{1}\right)-\psi\left(u_{2}\right)\right| .
\end{aligned}
$$

Agora usando a desigualdade de Cauchy- Schwarz segue que

$$
\begin{aligned}
\left\|f\left(u_{1}\right)-f_{1}\left(u_{2}\right)\right\| & \leq\left\|v\left(u_{1}\right)\right\|\left|\frac{\left\|v\left(u_{2}\right)\right\|^{2}-\left\|v\left(u_{1}\right)\right\|^{2}}{\left\|v\left(u_{1}\right)\right\|^{2}\left\|v\left(u_{2}\right)\right\|^{2}}\right| \\
& =\frac{\left|\left\langle v\left(u_{2}\right)-v\left(u_{1}\right), v\left(u_{2}\right)+v\left(u_{1}\right)\right\rangle\right|}{\left\|v\left(u_{1}\right)\right\|\left\|v\left(u_{2}\right)\right\|^{2}} \\
& \leq \frac{\left\|v\left(u_{1}\right)+v\left(u_{2}\right)\right\|}{\left\|v\left(u_{1}\right)\right\|\left\|v\left(u_{2}\right)\right\|^{2}}\left\|v\left(u_{1}\right)-v\left(u_{2}\right)\right\| \\
& \leq k_{2}\left\|u_{1}-u_{2}\right\|,
\end{aligned}
$$

já que $v$ é localmente Lipschitziana. Por outro lado, temos que

$$
\begin{aligned}
\left\|f_{1}\left(u_{2}\right)-f\left(u_{2}\right)\right\| & =\left\|\frac{v\left(u_{1}\right)}{\left\|v\left(u_{2}\right)\right\|^{2}}-\frac{v\left(u_{2}\right)}{\left\|v\left(u_{2}\right)\right\|^{2}}\right\| \\
& \leq \frac{\delta^{2}}{16 \varepsilon^{2}}\left\|v\left(u_{1}\right)-v\left(u_{2}\right)\right\| \\
& \leq k_{3}\left\|u_{1}-u_{2}\right\|
\end{aligned}
$$

e, como

$$
\frac{\delta}{4 \varepsilon}\left|\psi\left(u_{1}\right)-\psi\left(u_{2}\right)\right| \leq k_{1}\left\|u_{1}-u_{2}\right\|
$$

concluímos que 


$$
\left\|\Phi\left(u_{1}\right)-\Phi\left(u_{2}\right)\right\| \leq C\left\|u_{1}-u_{2}\right\|
$$

onde $C=k_{1}+k_{2}+k_{3}$. Portanto $\Phi$ é localmente Lipschitziana.

Considere agora o seguinte problema de Cauchy em espaços de Banach,

$$
(P)_{u} \quad\left\{\begin{aligned}
\frac{d}{d t} \sigma(t, u) & =\Phi(\sigma(t, u), \\
\sigma(0, u) & =u .
\end{aligned}\right.
$$

Como $\Phi$ é localmente Lipschitziana, temos que, para cada $u \in X$, o problema acima tem uma única solução contínua $\sigma(\cdot, u)$ definida para $t$ em um intervalo maximal $\left(t_{u}^{-}, t_{u}^{+}\right)$.

Afirmação B.1. $t_{u}^{ \pm}= \pm \infty$.

De fato, seja $\sigma$ a solução de $(P)_{u}$ e suponhamos que $t_{u}^{+}<\infty$. Considere também uma sequência $\left(t_{n}\right) \subset\left(-\infty, t_{u}^{+}\right)$tal que $t_{n} \rightarrow t_{u}^{+}$. Logo da limitação de $\Phi$, temos que

$$
\left\|\sigma\left(t_{m}, u\right)-\sigma\left(t_{n}, u\right)\right\|=\left\|\int_{t_{n}}^{t_{m}} \frac{d}{d \xi} \sigma(\xi, u) d \xi\right\|=\left\|\int_{t_{n}}^{t_{m}} \Phi(\sigma(\xi, u)) d \xi\right\| \leq C\left|t_{m}-t_{n}\right| .
$$

Como $\left(t_{n}\right) \subset \mathbb{R}$ é uma sequência de Cauchy, então $\left(\sigma\left(t_{n}, u\right)\right)$ também o é. Daí

$$
\lim _{n \rightarrow \infty} \sigma\left(t_{n}, u\right)=\tilde{u} \in X .
$$

Considerando o problema de Cauchy em espaços de Banach

$$
(P)_{\tilde{u}}\left\{\begin{aligned}
\frac{d}{d t} \sigma(t, u) & =\Phi(\sigma(t, u), \\
\sigma\left(t_{u}^{+}, u\right) & =\tilde{u},
\end{aligned}\right.
$$

podemos usar o Teorema de Picard para estender $\sigma$ em um intervalo do tipo $\left(t_{u}^{+}-k_{1}, t_{u}^{+}+k_{1}\right)$, contradizendo a maximalidade de $t_{u}^{+}$. A prova para $t_{u}^{-}$a ideia é totalmente análoga.

A dependência contínua de soluções de $(P)_{u}$ com relação aos dados iniciais implica que $\sigma \in C(\mathbb{R} \times$ $X, X)$. Desse modo, podemos definir a deformação

$$
\eta:[0,1] \times X \rightarrow X \quad \text { tal que } \quad \eta(t, u)=\sigma(\delta t, u) .
$$

Verifiquemos as condições (1) - (3). Pela própria definição da função $\eta$ temos que $\eta(0, u)=u$ para todo $u \in X$. Logo $\eta$ satisfaz (1). Para verificar (2), observe que $\Phi \equiv 0$ em $X \backslash A$ e portanto $\sigma(t, u)=u$ é solução de $(P)_{u}$, segue que $\eta(t, u)=u$ para todo $t \in \mathbb{R}$.

Para verificarmos (3) seja $t>0$ e $u \in X$. Pelo Teorema Fundamental do Cálculo temos que

$$
\begin{aligned}
\|\sigma(\delta t, u)-u\| & =\left\|\int_{0}^{\delta t} \frac{d}{d s} \sigma(s, u) d s\right\| \\
& \leq \int_{0}^{\delta t}\left\|\frac{d}{d s} \sigma(s, u)\right\| d s \\
& =\int_{0}^{\delta t}\|\Phi(\sigma(s, u))\| d s \\
& \leq \delta t .
\end{aligned}
$$


Logo, para todo $t \in[0,1]$ temos

$$
\|\sigma(\delta t, u)-u\| \leq \delta
$$

Assim,

$$
\min _{t \in[0,1]}\|\sigma(\delta t, u)-u\| \leq \delta,
$$

verificando que, para todo $u \in S, \sigma(\delta t, u) \in S_{\delta}$. Dessa forma $\sigma(\delta, S) \subseteq S_{\delta}$. Note que se $\sigma(t, u) \notin$ $A, \psi(\sigma(t, u))=0$ e consequentemente $\frac{d}{d t} I(\sigma(t, u))=0$. Caso contrário,

$$
\begin{aligned}
\frac{d}{d t} I(\sigma(t, u)) & =I^{\prime}(\sigma(t, u)) \frac{d}{d t} \sigma(t, u) \\
& =I^{\prime}(\sigma(t, u)) \Phi(\sigma(t, u)) \\
& =-\frac{\psi(\sigma(t, u))}{\|v(\sigma(t, u))\|} I^{\prime}(\sigma(t, u)) v(\sigma(t, u)) .
\end{aligned}
$$

Como $\psi$ é não negativa e de $(D 2)$, temos que

$$
I^{\prime}(\sigma(t, u)) v(\sigma(t, u)) \geq 0
$$

$\log 0$

$$
\frac{d}{d t} I(\sigma(t, u)) \leq 0 .
$$

Assim concluímos que $I(\eta(\cdot, u))$ é não crescente para todo $u \in X$. Tomando agora $u \in I^{c+\varepsilon} \cap S$, vamos dividir a prova em dois passos:

Passo 1: Existe $t_{0} \in[0, \delta)$ tal que $I\left(\eta\left(t_{0}, u\right)\right)<c-\varepsilon$.

Como $I(\eta(\cdot, u))$ é não-crescente, tem-se que

$$
I(\eta(t, u))<c-\varepsilon, \forall t \geq t_{0}
$$

e portanto

$$
\eta(1, u)=\sigma(\delta, u) \in I^{c-\varepsilon} .
$$

Como $\sigma(\delta, S) \subseteq S_{\delta}$ obtemos que

$$
\eta(1, u)=\sigma(\delta, u) \in I^{c-\varepsilon} \cap S_{\delta} .
$$

Passo 2: Para todo $t \in[0, \delta)$ temos,

$$
c-\varepsilon \leq I(\sigma(t, u)) \leq I(\sigma(0, u))=I(u) \leq c+\varepsilon .
$$

Logo

$$
\sigma(t, u) \in B
$$

Assim, usando que $\psi \equiv 1$ em $B,(D 1),(D 2)$ e (B.1), obtemos 


$$
\begin{aligned}
I(\sigma(\delta, u)) & =I(\sigma(0, u))+\int_{0}^{\delta} \frac{d}{d s} I(\sigma(s, u)) d s \\
& =I(u)+\int_{0}^{\delta} I^{\prime}(\sigma(s, u)) \Phi(\sigma(s, u)) d s \\
& =I(u)-\int_{0}^{\delta} I^{\prime}(\sigma(s, u)) \psi(\sigma(s, u)) \frac{v(\sigma(s, u))}{\|v(\sigma(s, u))\|} d s \\
& =I(u)-\int_{0}^{\delta} \frac{I^{\prime}(\sigma(s, u)) v(\sigma(s, u))}{\|v(\sigma(s, u))\|} d s \\
& \leq I(u)-\int_{0}^{\delta} \frac{\left\|I^{\prime}(\sigma(s, u))\right\|^{2}}{\|v(\sigma(s, u))\|} d s \\
& \leq I(u)-\frac{1}{2} \int_{0}^{\delta}\left\|I^{\prime}(\sigma(s, u))\right\| d s \\
& \leq I(u)-\frac{1}{2} \int_{0}^{\delta} \frac{4 \epsilon}{\delta} d s \\
& =I(u)-2 \varepsilon \\
& \leq c-\varepsilon .
\end{aligned}
$$

verificando assim o item (3) e concluindo a prova do Lema.

\section{B.2 O Teorema do Passo da Montanha}

O Teorema do Passo da Montanha, devido a Ambrosetti-Rabinowitz [3], é uma importante ferramenta para obtenção de pontos críticos para funcionais $I \in C^{1}(X, \mathbb{R})$. Conforme veremos mais em diante, é possível escolher $I$ de tal forma que seus pontos críticos sejam soluções de certas equações diferencias parciais.

Como estamos interessados em obter pontos críticos para um dado funcional $I \in C^{1}(X, \mathbb{R})$, precisamos provar alguma propiedade de compacidade para o mesmo.

Dizemos que $I \in C^{1}(X, \mathbb{R})$ satisfaz a condição de Palais-Smale no nível $c \in \mathbb{R}$ que denotaremos por $(P S)_{c}$ se toda sequência $\left(u_{n}\right) \subseteq X$ satisfazendo,

$$
\lim _{n \rightarrow \infty} I\left(u_{n}\right)=c \quad \text { e } \quad \lim _{n \rightarrow \infty}\left\|I^{\prime}\left(u_{n}\right)\right\|_{X^{\prime}}=0,
$$

possui subsequência convergente. A uma sequência $\left(u_{n}\right)$ cumprindo (B.4), chamamos de sequência de Palais- Smale no nível $c$.

A condição de compacidade que usaremos, a apresentada acima, se deva a Brezis e Niremberg (ver [7]). Sua versão original foi introduzida por Palais-Smale.

Proposição B.1. Seja $I \in C^{1}(X, \mathbb{R})$ tal que $I(0)=0 e$,

$\left(I_{1}\right)$ existem $\rho, \alpha>0$ tais que $\left.I\right|_{\partial B_{\rho}(0)} \geq \alpha$;

$\left(I_{2}\right)$ existe $e \in X$ tal que $\|$ e $\|_{X}>\rho$ e $I(e)<0$.

Seja

$$
c:=\inf _{\gamma \in \Gamma t \in[0,1]} \max _{t} I(\gamma(t))
$$


onde

$$
\Gamma:=\{\gamma \in C([0,1], X): \gamma(0)=0 \quad \text { e } \gamma(1)=e\}
$$

Então, dado $\varepsilon>0$, existe $u \in X$ tal que

(i) $u \in I^{-1}([c-2 \varepsilon, c+2 \varepsilon])$;

(ii) $\left\|I^{\prime}(u)\right\|_{X^{\prime}} \leq 2 \varepsilon$.

Demonstração. Seja $e \in X$ dado por $\left(I_{2}\right)$ e $\gamma \in \Gamma$. Então $e \notin B_{\rho}(0)$ e, como $\gamma \in \Gamma$, existe $t_{0} \in[0,1]$ tal que $\gamma\left(t_{0}\right) \in \partial B_{\rho}(0)$. Assim $\gamma([0,1]) \cap \partial B_{\rho}(0) \neq \emptyset$. Logo, por $\left(I_{1}\right)$, temos que

$$
\max _{t \in[0,1]} I(\gamma(t)) \geq \inf _{w \in \partial B_{\rho}(0)} I(w) \geq \alpha
$$

Tomando o ínfimo para $\gamma \in \Gamma$, concluímos que $c \geq \alpha \geq 0$.

Suponha, por contradição, que a proposição seja falsa. Então existe $\varepsilon>0$ tal que $\left\|I^{\prime}(u)\right\|_{X^{\prime}}>$ $2 \varepsilon$ para todo $u \in I^{-1}([c-2 \varepsilon, c+2 \varepsilon])$.

Observe que a afirmação acima permanece válida se subtituirmos $\varepsilon$ por $\varepsilon_{0}$ tal que $0<\varepsilon_{0}<\varepsilon$. Logo, podemos supor que $\varepsilon$ é pequeno de modo que $c-2 \varepsilon>0$. Estamos então nas hipóteses do Lema B.1, considerando $S=X$ e $\delta=2$. Assim, existe uma função contínua $\eta:[0,1] \times X \rightarrow X$ satisfazendo:

(i) $\eta(1, u)=u, \forall u \notin I^{-1}([c-2 \varepsilon, c+2 \varepsilon])$;

(ii) $\eta\left(1, I^{c+\varepsilon}\right) \subseteq I^{c-\varepsilon}$.

Pela definição de $c$, existe $\tilde{\gamma} \in \Gamma$ tal que

$$
\max _{t \in[0,1]} I(\tilde{\gamma}) \leq c+\varepsilon
$$

Defina agora $h:[0,1] \rightarrow X$ por

$$
h(t)=\eta(1, \tilde{\gamma}(t)) .
$$

Observe que $h \in C([0,1], X)$ pois $\eta \in C([0,1] \times X, X)$. Como $\tilde{\gamma} \in \Gamma$, temos que, $\tilde{\gamma}(0)=0, \tilde{\gamma}(1)=e$ e além disso, como $I(e)<c-2 \varepsilon$, segue de $(i)$ que

$$
h(0)=\eta(1, \gamma(0))=\eta(1,0)=0
$$

e

$$
h(1)=\eta(1, \tilde{\gamma}(1))=\eta(1, e)=e,
$$

onde concluímos que $h \in \Gamma$. Assim, temos que

$$
c \leq \max _{t \in[0,1]} I(h(t)) .
$$

Usando (ii) e (B.5) obtemos

$$
h(t)=\eta(1, \tilde{\gamma}(t)) \in I^{c-\varepsilon}, \text { para todo } t \in[0,1]
$$


Desta forma

$$
\max _{t \in[0,1]} I(h(t)) \leq c-\varepsilon
$$

Logo, de (B.6) e (B.7), concluímos que

$$
c \leq \max _{t \in[0,1]} I(h(t)) \leq c-\varepsilon,
$$

o que é um absurdo.

Corolário B.1. Sob as hipóteses da Proposição B.1, existe uma sequência de Palais- Smale no nível c para $I$.

Demonstração. Note que pela Proposição $B .1$, para cada $\varepsilon_{n}=\frac{1}{n}$, existe $u_{n} \in X$ de modo que

(i) $u_{n} \in I^{-1}([c-2 / n, c+2 / n])$;

(ii) $\left\|I^{\prime}\left(u_{n}\right)\right\|_{X^{\prime}} \leq 2 / n$.

Então,

$$
\lim _{n \rightarrow \infty} I\left(u_{n}\right)=c \text { e } \lim _{n \rightarrow \infty}\left\|I^{\prime}\left(u_{n}\right)\right\|_{X^{\prime}}=0,
$$

e portanto existe uma sequência de Palais-Smale no nível $c$.

Agora estamos em condições de demonstrar o

Teorema B.1 (Teorema do Passo da Montanha). Seja $X$ um espaço de Banach e $I \in C^{1}(X, \mathbb{R})$ tal que $I(0)=0 e$

$\left(I_{1}\right)$ existem $\rho, \alpha>0$ tais que $\left.I\right|_{\partial B_{\rho}(0)} \geq \alpha$;

$\left(I_{2}\right)$ existe $e \in X$ tal que $\|e\|_{X}>\rho$ e $I(e)<0$.

Suponha que I satisfaz $(P S)_{c}$ com

$$
c:=\inf _{\gamma \in \Gamma} \max _{t \in[0,1]} I(\gamma(t))
$$

onde $\Gamma:=\{\gamma \in C([0,1], X), \gamma(0)=0$ e $\gamma(1)=e\}$. Então existe $u \neq 0$ tal que $I(u)=c$ e $I^{\prime}(u)=0$.

Demonstração. Pelo Corolário B.1 existe uma sequência de Palais-Smale $\left(u_{n}\right) \subseteq X$ no nível c. Como $I$ satisfaz $(P S)_{c}$, a menos de subsequência, $u_{n} \rightarrow u \in X$. Como $I \in C^{1}(X, \mathbb{R})$, devemos necessariamente ter $I(u)=c$ e $I^{\prime}(u)=0$. logo $c$ é um valor crítico de $I$. Além disso, como $I(0)=0$ e $I(u)=c>0$, devemos ter $u \not \equiv 0$. 


\section{Apêndice}

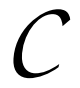

\section{Sub e supersolução}

Antes de enunciarmos o Teorema de Sub- Supersolução, definamos sub e supersolução para o problema

$$
\left\{\begin{aligned}
-\Delta u & =f(u), & & x \in \Omega, \\
u & =0, & & x \in \partial \Omega,
\end{aligned}\right.
$$

onde $\Omega \subset \mathbb{R}^{N}, N \geq 1$, é um domínio regular e $f: \mathbb{R} \rightarrow \mathbb{R}$ uma função de clase $C^{\alpha}$.

Quando falamos de solução de (C.1) estamos nos referindo, a menos que se diga algo contrário, à solução clássica, isto é, uma função $u \in C^{2}(\bar{\Omega})$ que satisfaz $(C .1)$.

Definição C.0.1. Uma função $\underline{U} \in C^{2}(\bar{\Omega})$ é dita uma subsolução do problema $(C .1)$ se

$$
\left\{\begin{aligned}
-\Delta \underline{U} & \leq f(\underline{U}), & & x \in \Omega, \\
\underline{U} & \leq 0, & & x \in \partial \Omega .
\end{aligned}\right.
$$

Definição C.0.2. Uma função $\bar{U} \in C^{2}(\bar{\Omega})$ é dita uma supersolução do problema (C.1) se

$$
\left\{\begin{aligned}
-\Delta \bar{U} & \geq f(\bar{U}), & & x \in \Omega, \\
\bar{U} & \geq 0, & & x \in \partial \Omega .
\end{aligned}\right.
$$

Teorema C.1. Suponhamos que o problema (C.1) possua uma subsolução $\underline{U}$ e uma supersolução $\bar{U}$, com $\underline{U} \leq \bar{U}$ em $\Omega$. Suponhamos, ainda, que $f: \mathbb{R} \rightarrow \mathbb{R}$ seja de classe $C^{\alpha} e$

$$
f\left(t_{1}\right)-f\left(t_{2}\right) \geq-k\left(t_{1}-t_{2}\right)
$$

para alguma constante $k \geq 0$ e para todo $t_{1} \geq t_{2},\left|t_{1}\right|,\left|t_{2}\right| \leq \max \left\{\|\underline{U}\|_{\infty},\|\bar{U}\|_{\infty}\right\}$. Então o problema (C.1) possui soluções $U, V \in C^{2, \alpha}(\bar{\Omega})$ tai que $\underline{U} \leq U \leq V \leq \bar{U}$. Além disso, qualquer solução u de $(C .1)$ com $\underline{U} \leq u \leq \bar{U}$ é tal que $U \leq u \leq V$, ou seja, $U$ é solução mínima e $V$ é solução máxima com respeito ao intervalo $[\underline{U}, \bar{U}]$.

Demonstração. Inicialmente, demonstraremos a existência de solução para o problema em questão. Para 
tanto, consideremos a função

$$
g(t)=f(t)+k t
$$

onde $k \geq 0$ e $\mid t \| \leq \max \left\{\|\underline{U}\|_{\infty},\|\bar{U}\|_{\infty}\right\}$. Note que, por (C.4), $g$ é crescente neste intervalo. Definamos, indutivamente, uma sequência de funções $u_{n} \in C^{2}(\bar{\Omega})$ por $u_{0}=\underline{U}$ e, para todo $n \geq 1$, $u_{n}$ é a solução única do problema linear

$$
\left\{\begin{aligned}
-\Delta u_{n}+k u_{n} & =g\left(u_{n-1}\right), & & x \in \Omega, \\
u_{n} & =0, & & x \in \partial \Omega .
\end{aligned}\right.
$$

Afirmação C.1. A função $g\left(u_{n-1}\right) \in C^{\alpha}(\bar{\Omega})$.

Com efeito, sendo $u_{n} \in C^{2}(\bar{\Omega})$ temos, pela Desigualdade do Valor Médio (ver Apêndice $A$, Teorema $A .3)$, que existe $M>0$ tal que

$$
\left|u_{n-1}(x)-u_{n-1}(y)\right| \leq M|x-y|
$$

o que implica

$$
\begin{aligned}
\frac{\left|g\left(u_{n-1}\right)(x)-g\left(u_{n-1}\right)(y)\right|}{|x-y|^{\alpha}} & =\frac{\left|f\left(u_{n-1}\right)(x)+k u_{n-1}(x)-f\left(u_{n-1}\right)(y)-k u_{n-1}(y)\right|}{|x-y|^{\alpha}} \\
& \leq \frac{\left|f\left(u_{n-1}\right)(x)-f\left(u_{n-1}\right)(y)\right|}{|x-y|^{\alpha}}+k \frac{\mid u_{n-1}(x)-u_{n-1}(y)}{|x-y|^{\alpha}} \\
& \leq \frac{\left|f\left(u_{n-1}\right)(x)-f\left(u_{n-1}\right)(y)\right|}{|x-y|^{\alpha}}+k M \frac{|x-y|}{|x-y|^{\alpha}} \\
& =\frac{\left|f\left(u_{n-1}\right)(x)-f\left(u_{n-1}\right)(y)\right|}{|x-y|^{\alpha}}+k M|x-y|^{1-\alpha} .
\end{aligned}
$$

Logo,

$$
\begin{aligned}
\sup _{x \neq y} \frac{\left|g\left(u_{n-1}\right)(x)-g\left(u_{n-1}\right)(y)\right|}{|x-y|^{\alpha}} & \leq \sup _{x \neq y} \frac{\left|f\left(u_{n-1}\right)(x)-f\left(u_{n-1}\right)(y)\right|}{|x-y|^{\alpha}} \\
& +k M \sup _{x \neq y}|x-y|^{1-\alpha} \\
& <\infty
\end{aligned}
$$

Uma vez que $g\left(u_{n-1}\right) \in C^{\alpha}(\bar{\Omega})$, pelo Teorema de Schauder (ver Apêndice $A$, Teorema A.11), o problema $(C .6)$ possui uma única solução $u_{n} \in C^{2, \alpha}(\bar{\Omega})$.

Afirmação C.2. $\underline{U}=u_{0} \leq u_{1} \leq \cdots \leq u_{n} \leq u_{n+1} \leq \cdots \leq \bar{U}$.

De fato, mostremos, primeiramente, que $\underline{U}=u_{0} \leq u_{1}$ em $\Omega$. Como $\underline{U}$ é subsolução de $(C .1)$, temos que

$$
\left\{\begin{aligned}
-\Delta \underline{U} & \leq f(\underline{U}), & & x \in \Omega \\
\underline{U} & \leq 0, & & x \in \partial \Omega .
\end{aligned}\right.
$$

Se $k \geq 0$, então

$$
\left\{\begin{array}{rlrl}
-\Delta \underline{U}+k \underline{U} \leq f(\underline{U})+k \underline{U}, & & x \in \Omega, \\
\underline{U} & \leq 0, & & x \in \partial \Omega
\end{array}\right.
$$

isto é

$$
\left\{\begin{aligned}
-\Delta \underline{U}+k \underline{U} \leq g(\underline{U}), & & x \in \Omega, \\
\underline{U} \leq 0, & & x \in \partial \Omega .
\end{aligned}\right.
$$


Sendo $u_{1}$ solução de $(C .6)$, segue

$$
\left\{\begin{aligned}
-\Delta u_{1}+k u_{1} & =g(\underline{U}), & & x \in \Omega \\
u_{1} & =0, & & x \in \partial \Omega,
\end{aligned}\right.
$$

daí

$$
\left\{\begin{aligned}
-\Delta \underline{U}+k \underline{U} \leq-\Delta u_{1}+k u_{1}, & & x \in \Omega, \\
\underline{U} \leq u_{1}, & & x \in \partial \Omega
\end{aligned}\right.
$$

implica em

$$
\left\{\begin{aligned}
-\Delta\left(u_{1}-\underline{U}\right)+k\left(u_{1}-\underline{U}\right) & \geq 0, & & x \in \Omega, \\
u_{1}-\underline{U} & \geq 0, & & x \in \partial \Omega .
\end{aligned}\right.
$$

Pelo princípio do máximo (ver apêndice $A$, Teorema $A .4$ ), $u_{1}-\underline{U} \geq 0$, em $\Omega$, ou seja, $\underline{U} \leq u_{1}$ em $\Omega$. Agora mostraremos que $u_{1} \leq \bar{U}$ em $\Omega$. Sendo $\bar{U}$ supersolução de $(C .1)$, temos

$$
\left\{\begin{array}{rlrl}
-\Delta \bar{U} & \geq f(\bar{U}), & & x \in \Omega \\
\bar{U} \geq 0, & & x \in \partial \Omega .
\end{array}\right.
$$

Se $k \geq 0$, então

$$
\left\{\begin{aligned}
-\Delta \bar{U}+k \bar{U} & \geq f(\bar{U})+k \bar{U}, & & x \in \Omega \\
\bar{U} & \geq 0, & & x \in \partial \Omega
\end{aligned}\right.
$$

ou seja,

$$
\left\{\begin{aligned}
-\Delta \bar{U}+k \bar{U} & \geq g(\bar{U}), & & x \in \Omega \\
\bar{U} & \geq 0, & & x \in \partial \Omega .
\end{aligned}\right.
$$

Por $g$ ser crescente, segue que $g(\underline{U}) \leq g(\bar{U})$ e de $(C .7)$ obtemos

$$
\left\{\begin{aligned}
-\Delta u_{1}+k u_{1} & \leq g(\bar{U}), & & x \in \Omega \\
u_{1} & =0, & & x \in \partial \Omega .
\end{aligned}\right.
$$

e assim,

$$
\left\{\begin{aligned}
-\Delta u_{1}+k u_{1} & \leq-\Delta \bar{U}+k \bar{U}, & & x \in \Omega \\
u_{1} & \leq \bar{U}, & & x \in \partial \Omega,
\end{aligned}\right.
$$

onde,

$$
\left\{\begin{aligned}
-\Delta\left(\bar{U}-u_{1}\right)+k\left(\bar{U}-u_{1}\right) & \geq 0, & & x \in \Omega, \\
\bar{U}-u_{1} & \leq 0, & & x \in \partial \Omega .
\end{aligned}\right.
$$

Logo, usando o princípio do máximo, $u_{1} \leq \bar{U}$ em $\Omega$.

Agora, suponhamos que $\underline{U}=u_{0} \leq \cdots u_{n-1} \leq u_{n} \leq \cdots \leq \bar{U}$ e mostremos que $\underline{U} \leq u_{n+1} \leq \bar{U}$. Considerando as equações que definem $u_{n}$ e $u_{n+1}$, temos

$$
\left\{\begin{aligned}
-\Delta u_{n}+k u_{n} & =g\left(u_{n-1}\right), & & x \in \Omega, \\
u_{n} & =0, & & x \in \partial \Omega
\end{aligned}\right.
$$


e

$$
\left\{\begin{aligned}
-\Delta u_{n+1}+k u_{n+1} & =g\left(u_{n}\right), & & x \in \Omega, \\
u_{n+1} & =0, & & x \in \partial \Omega .
\end{aligned}\right.
$$

Daí, subtraindo membro a membro as equações em $(C .8)$ e $(C .9)$, obtemos

$$
\left\{\begin{aligned}
-\Delta\left(u_{n+1}-u_{n}\right)+k\left(u_{n+1}-u_{n}\right) & =g\left(u_{n}\right)-g\left(u_{n-1}\right), & & x \in \Omega \\
u_{n+1}-u_{n} & =0, & & x \in \partial \Omega .
\end{aligned}\right.
$$

Como $g$ é crescente, $g\left(u_{n}\right)-g\left(u_{n-1}\right) \geq 0$ e, pelo princípio do máximo, $u_{n+1}-u_{n} \geq 0$ em $\Omega$, isto é $u_{n} \leq u_{n+1}$ em $\Omega$.

Evidentemente, $\underline{U} \leq u_{n+1}$ em $\Omega$. Com o raciocínio análogo para mostrar que $u_{1} \leq \bar{U}$ em $\Omega$, chega-se a $u_{n+1} \leq \bar{U}$ em $\Omega$. Portanto,

$$
\underline{U} \leq u_{n} \leq u_{n+1} \leq \bar{U} \quad \text { em } \Omega, \forall n \in \mathbb{N} .
$$

Por $(C .10)$ e em virtude da monotonicidade de $\left(u_{n}\right)$, existe uma função $U$, definida em $\bar{\Omega}$, tal que $u_{n} \rightarrow U$ pontualmente em $\bar{\Omega}$. E uma vez que $\underline{U}, \bar{U} \in C^{2}(\bar{\Omega})$, temos $\underline{U}, \bar{U} \in L^{p}(\Omega)$ para todo $p \geq 1$.

De $(C .10)$ segue

$$
\left|u_{n}\right| \leq \max \left\{\|\underline{U}\|_{\infty},\|\bar{U}\|_{\infty}\right\}=K \quad \text { em } \Omega, \forall n \in \mathbb{N} .
$$

Definamos a seguinte sequência

$$
h_{n}=\left|u_{n}-U\right|^{p}
$$

e note que

$$
h_{n} \rightarrow 0
$$

pontualmente em $\bar{\Omega}$ e

$$
\left\{\begin{aligned}
\left|h_{n}\right| & \leq 2^{p}\left(|K|^{p}+|K|^{p}\right) \\
& =2^{p+1} K^{p} \text { em } \Omega, \forall n \in \mathbb{N}
\end{aligned}\right.
$$

onde $2^{p+1} K^{p} \in L^{1}(\Omega)$. Logo, aplicando o Teorema da Convergência Dominada de Lebesgue(ver apêndice $A$, Teorema A.7), temos

$$
\left\|u_{n}-U\right\|_{L^{p}(\Omega)}^{p}=\int_{\Omega}\left|u_{n}-U\right|^{p} d x \rightarrow 0
$$

ou seja,

$$
u_{n} \rightarrow U \text { em } L^{p}(\Omega) .
$$

Da existência de uma constante real $M>0$ tal que

$$
\left|u_{n}\right| \leq M \quad \text { em } \bar{\Omega}, \forall n \in \mathbb{N}
$$

e da continuidade de $g$, obtemos uma constante $C>0$ de modo que

$$
\mid g\left(u_{n}\right) \leq C \quad \text { em } \bar{\Omega}, \forall n \in \mathbb{N},
$$

onde segue que $g\left(u_{n}\right) \in L^{p}(\Omega)$. Além disso, $g\left(u_{n}\right) \rightarrow g(U)$ pontualmente em $\bar{\Omega}$ e, novamente, pelo Teorema da Convergência Dominada de Lebesgue temos 


$$
\left\|g\left(u_{n}\right)-g(U)\right\|_{L^{p}(\Omega)}^{p}=\int_{\Omega}\left|g\left(u_{n}\right)-g(U)\right|^{p} d x \rightarrow 0,
$$

isto é,

$$
g\left(u_{n}\right) \rightarrow g(U) \quad \text { em } L^{p}(\Omega) .
$$

Sendo $g\left(u_{n-1}\right) \in L^{p}(\Omega)$ satisfazendo $(C .6)$, então $u_{n} \in W^{2, p}(\Omega)$ (ver apêndice $A$ )e existe uma constante $C$, que não depende de $n$ tal que

$$
\left\|u_{n}-u_{m}\right\|_{W^{2, p}(\Omega)} \leq C\left\|g\left(u_{n-1}\right)-g\left(u_{m-1}\right)\right\|_{L^{p}(\Omega)} .
$$

Logo, $\left(u_{n}\right)$ é uma sequência de Cauchy em $W^{2, p}(\Omega)$, pois $\left(g\left(u_{n}\right)\right)$ é uma sequência de Cauchy em $L^{p}(\Omega)$. Por $W^{2, p}(\Omega)$ ser um espaço de Banach, existe $\tilde{U} \in W^{2, p}(\Omega)$ tal que $u_{n} \rightarrow \tilde{U}$ em $W^{2, p}(\Omega)$. além disso, por

$$
\left\|u_{n}-\tilde{U}\right\|_{L^{p}(\Omega)} \leq\left\|u_{n}-\tilde{U}\right\|_{W^{2, p}(\Omega)},
$$

segue que $u_{n} \rightarrow \tilde{U}$ em $L^{p}(\Omega)$. Assim, pela unicidade do limite, obtemos $U=\tilde{U}$ e daí,

$$
u_{n} \rightarrow U \text { em } W^{2, p}(\Omega)
$$

Tomando $p>N$, tem-se a imersão compacta $W^{2, p}(\Omega) \hookrightarrow C^{1, \alpha}(\bar{\Omega})$, para $\alpha=1-\frac{N}{p}$. Isso implica

$$
u_{n} \rightarrow U \quad \operatorname{em~} C^{1, \alpha}(\bar{\Omega})
$$

Pela estimativa de Schauder, obtemos

$$
\left\|u_{n}\right\|_{C^{2, \alpha}(\bar{\Omega})} \leq C\left\|g\left(u_{n-1}\right)\right\|_{C^{\alpha}(\bar{\Omega})}, \forall n \in \mathbb{N} .
$$

Consequentemente, $\left(u_{n}\right)$ é limitada em $C^{2, \alpha}(\bar{\Omega})$. Desde que $C^{2, \alpha}(\bar{\Omega}) \hookrightarrow C^{2}(\bar{\Omega})$ compactamente existe uma subsequência de $\left(u_{n}\right)$ que converge para $U$ em $C^{2}(\bar{\Omega})$. Como $\left(u_{n}\right)$ é monótona, a sequência toda converge para $U$ em $C^{2}(\bar{\Omega})$. Uma vez que

$$
\left\{\begin{aligned}
-\Delta u_{n+1}+k u_{n+1} & =f\left(u_{n}\right)+k u_{n}, & & x \in \Omega \\
u_{n+1} & =0, & & x \in \partial \Omega
\end{aligned}\right.
$$

passando ao limite, segue- se

$$
\left\{\begin{aligned}
-\Delta U+k U & =f(U)+k U, & & x \in \Omega \\
U & =0, & & x \in \partial \Omega
\end{aligned}\right.
$$

e daí

$$
\left\{\begin{aligned}
-\Delta U & =f(U), & & x \in \Omega \\
U & =0, & & x \in \partial \Omega .
\end{aligned}\right.
$$

Analogamente, obtemos uma sequência não-crescente $\left(v_{n}\right)$ tal que

$$
\underline{U} \leq \cdots \leq v_{n+1} \leq v_{n} \leq \cdots \leq v_{0}=\bar{U}
$$


de modo que $v_{n} \rightarrow V$ em $C^{2}(\bar{\Omega})$ e

$$
\left\{\begin{aligned}
-\Delta V & =f(V), & & x \in \Omega \\
V & =0, & & x \in \partial \Omega .
\end{aligned}\right.
$$

Além disso, $\underline{U} \leq U \leq V \leq \bar{U}$ em $\Omega$.

Mostraremos agora as existências das soluções minimal e maximal respectivamente com respeito ao intervalo $[\underline{U}, \bar{U}]$. Seja $u$ uma solução de $(C .1) \operatorname{com} \underline{U} \leq u \leq \bar{U}$ em $\Omega$. Considere o intervalo $[\underline{U}, u]$ e apliquemos o procedimento anterior para obter uma sequência não-decrescente em que

$$
\underline{U} \leq u_{1} \leq \cdots \leq u_{n} \leq \cdots \leq u
$$

com $u_{n} \rightarrow U$. Logo, $U \leq u$ é solução mínima com respeito a $[\underline{U}, \bar{U}]$. Analogamente, se demonstra a existência da solução maximal.

\section{C.1 Sub e supersolucão fraca}

O lema a seguir assegura, sob certas condições, a existência de pontos críticos para funcionais definidos em espaços de Banach reflexivos. Ele será usado na demonstração do Teorema de sub e supersolução fraca.

Lema C.1. Sejam $X$ um espaço de Banach reflexivo com norma $\|\cdot\|_{X}, M \subset X$ um subconjunto fechado na topologia fraca e $I: X \rightarrow \mathbb{R}$ um funcional limitado inferiormente satisfazendo:

$\left(I_{1}\right) I(u) \rightarrow \infty$ quando $\|u\|_{X} \rightarrow \infty, u \in M$, ou seja, I é coerciva;

$\left(I_{2}\right)$ toda sequência $\left(u_{n}\right) \subset M$ tal que $u_{n} \rightarrow u$ fracamente em $X$ satisfaz

$$
I(u) \leq \liminf _{n \rightarrow \infty} I\left(u_{n}\right)
$$

Então I atinge infimo em $M$.

Demonstração. Seja $\alpha=\inf \{I(u): u \in M\}$ e $\left(u_{n}\right)$ uma sequência minimizante em $M$, ou seja, tal que $I\left(u_{n}\right) \rightarrow \alpha$. Uma vez que $I$ é coercivo, temos que $\left(u_{n}\right)$ é limitado, pois caso contrário existiria uma subsequência $\left(u_{n_{j}}\right) \subset\left(u_{n}\right)$ tal que $\left\|u_{n_{j}}\right\| \rightarrow \infty$ e portanto $I\left(u_{n_{j}}\right) \rightarrow \infty$. Como $X$ é reflexivo, a menos de subsequência, existe $u \in X$ tal que $u_{n} \rightarrow u$ fracamente. Mas como $M$ é fracamente fechado, então $u \in M$. Usando a hipótese de que $I$ é fracamente semicontínuo inferiormente, temos que

$$
I(u) \leq \liminf _{n \rightarrow \infty} I\left(u_{n}\right)=\alpha
$$

e portanto $I$ atinge ínfimo em $M$.

Consideremos o problema

$$
(P 1) \quad\left\{\begin{aligned}
-\Delta u & =f(x, u), & & x \in \Omega \\
u & =0, & & x \in \partial \Omega,
\end{aligned}\right.
$$

onde $\Omega \subset \mathbb{R}^{N}$ é um domínio suave e limitado, $N \geq 3$ e $f: \Omega \times \mathbb{R} \rightarrow \mathbb{R}$ é uma função de Carathéodory. 
Uma solução fraca do problema $(P 1)$ é uma função $u \in H$ que satisfaz

$$
\int_{\Omega} \nabla u \nabla v d x=\int_{\Omega} f(x, u) v d x, \forall v \in H .
$$

As soluções fracas de $(P 1)$ são pontos críticos do funcional $I: H \rightarrow \mathbb{R}$, dado por

$$
I(u)=\frac{1}{2} \int_{\Omega}|\nabla u|^{2} d x-\int_{\Omega} F(x, u) d x
$$

onde

$$
F(x, s)=\int_{0}^{s} f(x, t) d t .
$$

Definição C.1. Dizemos que $\underline{u} \in H^{1}(\Omega)$ é uma subsolução fraca para o problema $(P 1)$ se $\underline{u} \leq 0$ em $\partial \Omega$ $e$

$$
\int_{\Omega} \nabla \underline{u} \nabla v d x \leq \int_{\Omega} f_{\lambda}(x, \underline{u}) v d x
$$

para todo $v \in H \cap L^{\infty}(\Omega), v \geq 0$. Analogamente $\bar{u} \in H^{1}(\Omega)$ é supersolução fraca para o problema $(P 1)$ se $\bar{u} \geq 0$ em $\partial \Omega e$

$$
\int_{\Omega} \nabla \bar{u} \nabla v d x \geq \int_{\Omega} f_{\lambda}(x, \bar{u}) v d x
$$

para todo $v \in H \cap L^{\infty}(\Omega), v \geq 0$.

Teorema C.2 (Teorema de sub e supersolução). Suponha que $\underline{u} \in H^{1}(\Omega)$ é uma subsolução fraca e que $\bar{u} \in H^{1}(\Omega)$ é uma supersolução fraca para o problema $\left(P_{\lambda}\right)$. Suponha ainda que existam constantes $\underline{c}, \bar{c} \in \mathbb{R}$ tais que $\underline{c} \leq \underline{u} \leq \bar{u} \leq \bar{c}$ em quase todo ponto de $\Omega$. Então o problema (P1) admite uma solução fraca $u \in H$ satisfazendo $\underline{u} \leq u \leq \bar{u}$ em quase todo ponto de $\Omega$.

Demonstração. Vamos considerar o funcional

$$
I(u)=\frac{1}{2} \int_{\Omega}|\nabla u|^{2} d x-\int_{\Omega} F(x, u) d x
$$

onde $F(x, s)=\int_{0}^{s} f(x, t) d t$, restrito ao conjunto

$$
M=\{u \in H ; \underline{u}(x) \leq u(x) \leq \bar{u}(x) \text { em quase todo ponto de } \Omega\} .
$$

Como por definição $\bar{u}, \underline{u} \in L^{\infty}(\Omega)$, então $M \subset L^{\infty}(\Omega)$. Observemos que, dado $u \in M$, temos que $|u(x)| \leq C_{1}$ em quase todo ponto de $\Omega$, onde $C_{1}=\max \{|\bar{c}|,|\underline{c}|\}$. Portanto, como $f(x, \cdot)$ é contínua, vale

$$
\begin{aligned}
|F(x, u(x))| & =\left|\int_{0}^{u(x)} f(x, t) d t\right| \\
& \leq \int_{0}^{u(x)}|f(x, t)| d t \\
& \leq \int_{0}^{C_{1}}|f(x, t)| d t \\
& =C_{2}
\end{aligned}
$$

em quase todo ponto de $\Omega$. 
Deseja-se garantir a existência de um mínimo local para o funcional $I$. Para isso, vamos verificar as hipóteses do Lema $C .1$ Sabemos que $H$ é reflexivo. Além disso, seja $\left(v_{n}\right) \subset M$ tal que $v_{n} \rightarrow v$ em $H$. A menos de subsequência,

$$
\left\{\begin{array}{l}
\left(v_{n}\right) \text { é limitada em } H, \\
v_{n} \rightarrow v \text { em } L^{2}(\Omega), \\
v_{n}(x) \rightarrow v(x) \text { em quase todo ponto de } \Omega
\end{array}\right.
$$

e portanto $\underline{u}(x) \leq v(x) \leq \bar{u}(x)$ em quase todo ponto de $\Omega$. Logo $v \in M$ e com isso $M$ é fechado.

Dados agora $u, v \in M$ e $t \in[0,1]$, temos que $(1-t) v+t u \in H$. Além disso, em quase todo ponto de $\Omega$ vale

$$
\begin{aligned}
t \underline{u} & \leq t u \leq t \bar{u} \\
(1-t) \underline{u} & \leq(1-t) v \leq(1-t) \bar{u} .
\end{aligned}
$$

Somando as desigualdades segue que

$$
\underline{u} \leq(1-t) v+t u \leq \bar{u} \quad \text { em quase todo ponto de } \Omega,
$$

onde concluímos que $M$ é convexo. Sendo $M$ fechado e convexo, concluímos que $M$ é fracamente fechado. Observemos que

$$
\begin{aligned}
I(u) & =\frac{1}{2} \int_{\Omega}|\nabla u|^{2} d x-\int_{\Omega} F(x, u) d x \\
& \geq \frac{1}{2}\|u\|^{2}-\int_{\Omega} C_{2} d x \\
& =\frac{1}{2}\|u\|^{2}-C_{2}|\Omega|,
\end{aligned}
$$

portanto $I$ é limitado inferiormente em $M$ e $I(u) \rightarrow \infty$ quando $\|u\| \rightarrow \infty$ em $M$.

Resta mostrar que $I$ é fracamente semi-contínuo inferiormente, ou seja, que $I$ satisfaz a condição $I_{2}$. Seja $\left(u_{n}\right) \subset M$ tal que $u_{n} \rightarrow u$ em $H$. Como a imersão $H \hookrightarrow L^{2}(\Omega)$ é compacta e $\left(u_{n}\right)$ é limitada,

$$
\begin{aligned}
u_{n_{j}} \rightarrow u & \text { em } L^{2}(\Omega), \\
u_{n_{j}}(x) \rightarrow u(x) & \text { em quase todo ponto de } \Omega,
\end{aligned}
$$

para alguma subsequência $\left(u_{n_{j}}\right) \subset\left(u_{n}\right)$.

Como $\left|F\left(x, u_{n}(x)\right)\right| \leq C_{2}$ uniformemente, pelo Teorema da Convergência Dominada,

$$
\lim _{n_{j} \rightarrow \infty} \int_{\Omega} F\left(x, u_{n_{j}}\right) d x=\int_{\Omega} \lim _{n_{j} \rightarrow \infty} F\left(x, u_{n_{j}}\right) d x=\int_{\Omega} F(x, u) d x .
$$

Segue então que

$$
\lim _{n \rightarrow \infty} \int_{\Omega} F\left(x, u_{n}\right) d x=\int_{\Omega} F(x, u) d x
$$

pois, caso contrário, existiria uma constante $d>0$ e uma subsequência $\left(u_{n_{k}}\right) \subset\left(u_{n}\right)$ tal que

$$
\left|\int_{\Omega} F\left(x, u_{n_{k}}\right) d x-\int_{\Omega} F(x, u) d x\right| \geq d .
$$

Mas $\left(u_{n_{k}}\right)$ também é limitada, e o mesmo argumento em (C.13) nos daria uma contradição. Usando 
agora o fato de que $\|\cdot\|^{2}$ é fracamente semi-contínua inferiormente e (C.14), segue que

$$
\begin{aligned}
\liminf _{n \rightarrow \infty} I\left(u_{n}\right) & =\liminf _{n \rightarrow \infty}\left(\frac{1}{2}\left\|u_{n}\right\|^{2}-\int_{\Omega} F\left(x, u_{n}\right) d x\right) \\
& \geq \liminf _{n \rightarrow \infty} \frac{1}{2}\left\|u_{n}\right\|^{2}+\liminf _{n \rightarrow \infty}\left(-\int_{\Omega} F\left(x, u_{n}\right) d x\right) \\
& =\liminf _{n \rightarrow \infty} \frac{1}{2}\left\|u_{n}\right\|^{2}-\limsup _{n \rightarrow \infty} \int_{\Omega} F\left(x, u_{n}\right) d x \\
& \geq \frac{1}{2}\|u\|^{2}-\int_{\Omega} F(x, u) d x \\
& =I(u) .
\end{aligned}
$$

Portanto, pelo Lema $(C .1)$,$I atinge mínimo em M$, que chamaremos de $u$.

Dados $\varphi \in H \cap L^{\infty}(\Omega)$ e $\varepsilon>0$, seja

$$
v_{\varepsilon}=\min \{\bar{u}, \max \{\underline{u}, u+\varepsilon \varphi\}\}=u+\varepsilon \varphi-\varphi^{\varepsilon}+\varphi_{\varepsilon},
$$

onde

$$
\begin{aligned}
& \varphi^{\varepsilon}=\max \{0, u+\varepsilon \varphi-\bar{u}\} \geq 0, \\
& \varphi_{\varepsilon}=-\min \{0, u+\varepsilon \varphi-\underline{u}\} \geq 0 .
\end{aligned}
$$

Observemos que $v_{\varepsilon} \leq \bar{u}$ e, nos pontos onde $\bar{u} \geq \max \{\underline{u}, u+\varepsilon \varphi\}, v_{\varepsilon} \geq \underline{u}$. Portanto $v_{\varepsilon} \in M$. Além disso, se $u+\varepsilon \varphi-\bar{u}>0$, o operado traço (ver definição no apêndice $A$ ), logo satisfaz

$$
0 \leq T\left(\varphi^{\varepsilon}\right)=T(u+\varepsilon \varphi-\bar{u})=T(u-\bar{u}) \leq 0 .
$$

Por outro lado, se $u+\varepsilon \varphi-\underline{u}<0$, então

$$
0 \leq T\left(\varphi_{\varepsilon}\right)=T(-u-\varepsilon \varphi+\underline{u})=T(\underline{u}-u) \leq 0 .
$$

Logo, $\varphi^{\varepsilon}, \varphi_{\varepsilon} \in H_{0}^{1}(\Omega) \cap L^{\infty}$.

Usando a hipótese de que $|F(x, u)| \leq C_{2}$, e a definição de diferenciabilidade mostra que $I$ é diferenciável na direção de $v_{\varepsilon}-u$. Como $u$ é mínimo em $M$, para $t \in(0,1)$,

$$
\frac{I\left(u+t\left(v_{\varepsilon}-u\right)\right)-I(u)}{t}=\frac{I\left((1-t) u+t v_{\varepsilon}\right)-I(u)}{t} \geq 0,
$$

onde $(1-t) u+t v_{\varepsilon} \in M$, visto que $M$ é convexo. Logo

$$
0 \leq \lim _{t \rightarrow 0^{+}} \frac{I\left(u+t\left(v_{\varepsilon}-u\right)\right)-I(u)}{t}=I^{\prime}(u)\left(v_{\varepsilon}-u\right)=\varepsilon I^{\prime}(u) \varphi-I^{\prime}(u) \varphi^{\varepsilon}+I^{\prime}(u) \varphi_{\varepsilon},
$$

onde se conclui que

$$
I^{\prime}(u) \varphi \geq \frac{1}{\varepsilon}\left[I^{\prime}(u) \varphi^{\varepsilon}-I^{\prime}(u) \varphi_{\varepsilon}\right]
$$

Como $\bar{u}$ é supersolução fraca, então

$$
I^{\prime}(\bar{u}) \varphi^{\varepsilon}=\int_{\Omega} \nabla \bar{u} \nabla \varphi^{\varepsilon}-\int_{\Omega} f(x, \bar{u}) \varphi^{\varepsilon} \geq 0 .
$$


Assim,

$$
\begin{aligned}
I^{\prime}(u) \varphi^{\varepsilon} & =I^{\prime}(\bar{u}) \varphi^{\varepsilon}+\left(I^{\prime}(u)-I^{\prime}(\bar{u})\right) \varphi^{\varepsilon} \\
& \geq\left(I^{\prime}(u)-I^{\prime}(\bar{u})\right) \varphi^{\varepsilon} \\
& =\int_{\Omega}\left\{\nabla(u-\bar{u}) \nabla \varphi^{\varepsilon}-(f(x, u)-f(x, \bar{u})) \varphi^{\varepsilon}\right\} \\
& =\int_{\tilde{\Omega}_{\varepsilon}}\{\nabla(u-\bar{u}) \nabla(u+\varepsilon \varphi-\bar{u})-(f(x, u)-f(x, \bar{u}))(u+\varepsilon \varphi-\bar{u})\}
\end{aligned}
$$

onde $\tilde{\Omega}_{\varepsilon}=\{x \in \Omega, u(x)+\varepsilon \varphi(x) \geq \bar{u}(x)\}$. Observe que nesse caso só calculamos a integral em $\tilde{\Omega}_{\varepsilon}$, pois se $x \notin \tilde{\Omega}_{\varepsilon}, \varphi^{\varepsilon}(x)=0$. Mas se $x \in \tilde{\Omega}_{\varepsilon}$ é tal que $u(x)=\bar{u}(x)$, então $\nabla(u(x)-\bar{u}(x))=0$ e $f(x, u(x))-$ $f(x, \bar{u}(x))=0$. Logo $(C .16)$ implica que

$$
I^{\prime}(u) \varphi^{\varepsilon} \geq \int_{\Omega_{\varepsilon}}\{\nabla(u-\bar{u}) \nabla(u+\varepsilon \varphi-\bar{u})-(f(x, u)-f(x, \bar{u}))(u+\varepsilon \varphi-\bar{u})\}
$$

onde $\Omega_{\varepsilon}=\{x \in \Omega ; u(x)+\varepsilon \varphi(x) \geq \bar{u}(x)>u(x)\}$. Sejam agora

$$
\begin{aligned}
& \Omega_{\varepsilon}^{+}=\left\{x \in \Omega_{\varepsilon} ; f(x, u(x))-f(x, \bar{u}(x)) \geq 0\right\}, \\
& \Omega_{\varepsilon}^{+}=\left\{x \in \Omega_{\varepsilon} ; f(x, u(x))-f(x, \bar{u}(x)) \leq 0\right\} .
\end{aligned}
$$

$\operatorname{Em} \Omega_{\varepsilon}^{+}$valem as seguintes desigualdades

$$
\begin{aligned}
(f(x, u)-f(x, \bar{u}))(u+\varepsilon \varphi-\bar{u}) & =(f(x, u)-f(x, \bar{u}))(u-\bar{u})+(f(x, u)-f(x, \bar{u})) \varepsilon \varphi \\
& \leq(f(x, u)-f(x, \bar{u})) \varepsilon \varphi \\
& \leq \varepsilon|f(x, u)-f(x, \bar{u})||\varphi|
\end{aligned}
$$

enquanto em $\Omega_{\varepsilon}^{-}$, como $|u-\bar{u}|=\bar{u}-u \leq \varepsilon \varphi$,

$$
\begin{aligned}
(f(x, u)-f(x, \bar{u}))(u+\varepsilon \varphi-\bar{u}) & \leq(f(x, u)-f(x, \bar{u}))(u-\bar{u}) \\
& \leq|f(x, u)-f(x, \bar{u})|(\bar{u}-u) \\
& \leq \varepsilon|f(x, u)-f(x, \bar{u})||\varphi| .
\end{aligned}
$$

Logo, por (C.18) e (C.19)

$$
\begin{aligned}
\int_{\Omega_{\varepsilon}}(f(x, u)-f(x, \bar{u}))(u+\varepsilon \varphi-\bar{u}) & =\int_{\Omega_{\varepsilon}^{+}}(f(x, u)-f(x, \bar{u}))(u+\epsilon \varphi-\bar{u})+ \\
& \int_{\Omega_{\varepsilon}^{-}}(f(x, u)-f(x, \bar{u}))(u+\varepsilon \varphi-\bar{u}) \\
& \leq \int_{\Omega_{\varepsilon}^{+}} \varepsilon|f(x, u)-f(x, \bar{u})||\varphi|+ \\
& \int_{\Omega_{\varepsilon}^{-}} \varepsilon|f(x, u)-f(x, \bar{u}) \| \varphi| \\
& =\varepsilon \int_{\Omega_{\varepsilon}}|f(x, u)-f(x, \bar{u}) \| \varphi| .
\end{aligned}
$$


Além disso,

$$
\begin{aligned}
\int_{\Omega_{\varepsilon}} \nabla(u-\bar{u}) \nabla(u+\varepsilon \varphi-\bar{u}) & =\int_{\Omega_{\varepsilon}} \nabla(u-\bar{u}) \nabla(u-\bar{u})+\varepsilon \int_{\Omega_{\varepsilon}} \nabla(u-\bar{u}) \varphi \\
& \geq \varepsilon \int_{\Omega_{\varepsilon}} \nabla(u-\bar{u}) \varphi .
\end{aligned}
$$

Por $(C .17),(C .20)$ e $(C .21)$, temos

$$
\frac{I^{\prime}(u) \varphi^{\varepsilon}}{\varepsilon} \geq \int_{\Omega_{\varepsilon}} \nabla(u-\bar{u}) \varphi-\int_{\Omega_{\varepsilon}}|f(x, u)-f(x, \bar{u})||\varphi| .
$$

Como $\left|\Omega_{\varepsilon}\right| \rightarrow 0$ quando $\varepsilon \rightarrow 0$ e os integrandos acima não depende de $\varepsilon$,

$$
\liminf _{\varepsilon \rightarrow 0^{+}} \frac{I^{\prime}(u) \varphi^{\varepsilon}}{\varepsilon} \geq 0 .
$$

De maneira análoga, prova-se que

$$
\limsup _{\varepsilon \rightarrow 0^{+}} \frac{I^{\prime}(u) \varphi_{\varepsilon}}{\varepsilon} \leq 0 .
$$

Logo, por (C.15),

$$
\begin{aligned}
I^{\prime}(u) \varphi & \geq \liminf _{\varepsilon \rightarrow 0}\left(\frac{I^{\prime}(u) \varphi^{\varepsilon}}{\varepsilon}-\frac{I^{\prime}(u) \varphi_{\varepsilon}}{\varepsilon}\right) \\
& \geq \liminf _{\varepsilon \rightarrow 0} \frac{I^{\prime}(u) \varphi^{\varepsilon}}{\varepsilon}+\liminf _{\varepsilon \rightarrow 0} \frac{-I^{\prime}(u) \varphi_{\varepsilon}}{\varepsilon} \\
& =\liminf _{\varepsilon \rightarrow 0} \frac{I^{\prime}(u) \varphi^{\varepsilon}}{\varepsilon}-\limsup _{\varepsilon \rightarrow 0} \frac{I^{\prime}(u) \varphi_{\varepsilon}}{\varepsilon} \\
& =0 .
\end{aligned}
$$

Portanto, $I^{\prime}(u) \varphi \geq 0$ para todo $\varphi \in H(\Omega) \cap L^{\infty}(\Omega)$. Invertendo o sinal de $\varphi$, temos que $I^{\prime}(u) \varphi \leq 0$. Logo

$$
I^{\prime}(u) \varphi=0
$$

para todo $\varphi \in H \cap L^{\infty}(\Omega)$. Dada agora $\varphi \in H$ consideremos $\left(\varphi_{n}\right) \subset C_{0}^{\infty}(\Omega)$ tal que $\varphi_{n} \rightarrow \varphi$ em $H$. Temos que,

$$
0=\lim _{n \rightarrow \infty} I^{\prime}(u) \varphi_{n}=I^{\prime}(u) \varphi
$$

e portanto $I^{\prime}(u)=0$. 


\section{Referências Bibliográficas}

[1] Ambrosetti, A., Brezis, H. and Cerami, G. Combined Effects of Concave and Convex Nonlinearities in Some Elliptic Problems., Journal of Functional Analysis 122 (1994), 519-543.

[2] Ambrosetti, A. and Prodi, G., A Primer of Nonlinear Analysis., Cambridge University Press, New York, (1993).

[3] Ambrosetti, A. and Rabinowitz, P. H., Dual variational methods in critical point theory and applications., J. Functional Analysis 14 (1973), 349-381.

[4] Bartle, R.G., Elements of Integration and Lebesgue Measuare., Wiley Classics Library Edition Published, New York, (1995).

[5] Brezis, H. and Kamin, S., Sublinear elliptic equations in $\mathbb{R}^{n}$., Manuscripta Math. 74 (1992), 87-106.

[6] Brezis, H. and Kato T., Remarks on the Schrödinger operator with singular complex potentials., Jour. Math. Pures Appl. 58 (1983), 137-151.

[7] Brezis, H. and Nirenberg, L., $H^{1}$ versus $C^{1}$ local minimizers., C.R. Acad. Sci. Paris, 317 (1993), $465-472$

[8] Brezis, H., Functional Analysis, Sobolev Spaces and Partial Differential Equations., Springer - Verlag, (2010).

[9] Clark, D.C., A variant of the Ljusternik-Schnirelmann theory. Indiana Univ. Math. J. 22 (1972), $65-74$.

[10] de Figueiredo, D.G., Equações Elípticas não Lineares., $11^{\circ}$ Colóquio Brasileiro de Matemática, IMPA, Rio de Janeiro, (1997).

[11] de Figueiredo, D.G., Lectures on the Ekeland Variational Principle with Applications and Detours., Tata Inst. Fund. Res. Lectures Math. Phys. 81, Springer (1989) Zbl 0688.49011 MR 1019559

[12] de Figueiredo, D.G., Gossez, J.P and Ubilla, P., Multiplicity results for a family of semilinear elliptic problems under local superlinearity and sublinearity., J. Eur. Math. Soc 8 (2006), 269-286.

[13] de Paiva., Nonnegative solutions of elliptic problems with sublinear indefinite nonlinearity., Journal of Functional Analysis., 261 (2011), 2569-2586. 
[14] Elon, L.L., Análise Real Vol. 2., Coleção Matemática Universitária, IMPA, Rio de Janeiro, (2004).

[15] Elon, L.L., Espaços Métricos., Projeto Euclides, IMPA, Rio de Janeiro, (2005).

[16] Gilbarg, D. and Trundinger, N.S., Elliptic Partial Differential Equations of Second Order., Classics in Mathematics, Springer, $3^{a}$ Edição, New York, (2001).

[17] Kavian, O., Introduction à la Théorie des Points Critiques et Applications aux Problémes Elliptiques., Springer-Verlag, (1993).

[18] Lawrence, C.E., Partial Differential Equations., American Mathematical Society, U.S.A., 19 (1998).

[19] Lusternik L., Schnirelmann L., Méthodes Topologiques dans les Problèmes Variationnels., Francia, (1934).

[20] Mignot, F. and Puel, J. P., Sur une classe de problèmes nonlinéaires avc nonlinéarité positive, croissante, convexe., Comm. Partial Differential Equations 5 (1980), 791-836.

[21] Paul H.R., Minimax Methods in Critical Point Theory with Applications to Differential Eaquations., Expository lectures from the CBMS Regional Conference, Miami, 45 (1984).

[22] Struwe, M., Variational Methods. Springer (1990) Zbl 0746.49010 MR 1078018.

[23] Tarantelo G., On nonhomogeneus elliptic equations involving critical Sobolev exponent., Ann. Inst. H. Poincaré Anal. Non Linéaire 9 (1992), 281-304.

[24] Trudinger, N.S., On Harnack type inequalities and their application to quasilinear elliptic equations., Comm. Pure Appl. Math., 20 (1967), 721-747.

[25] Willem, M., Minimax Theorems., Volume 24, Birkhauser, Boston, 1996. 